
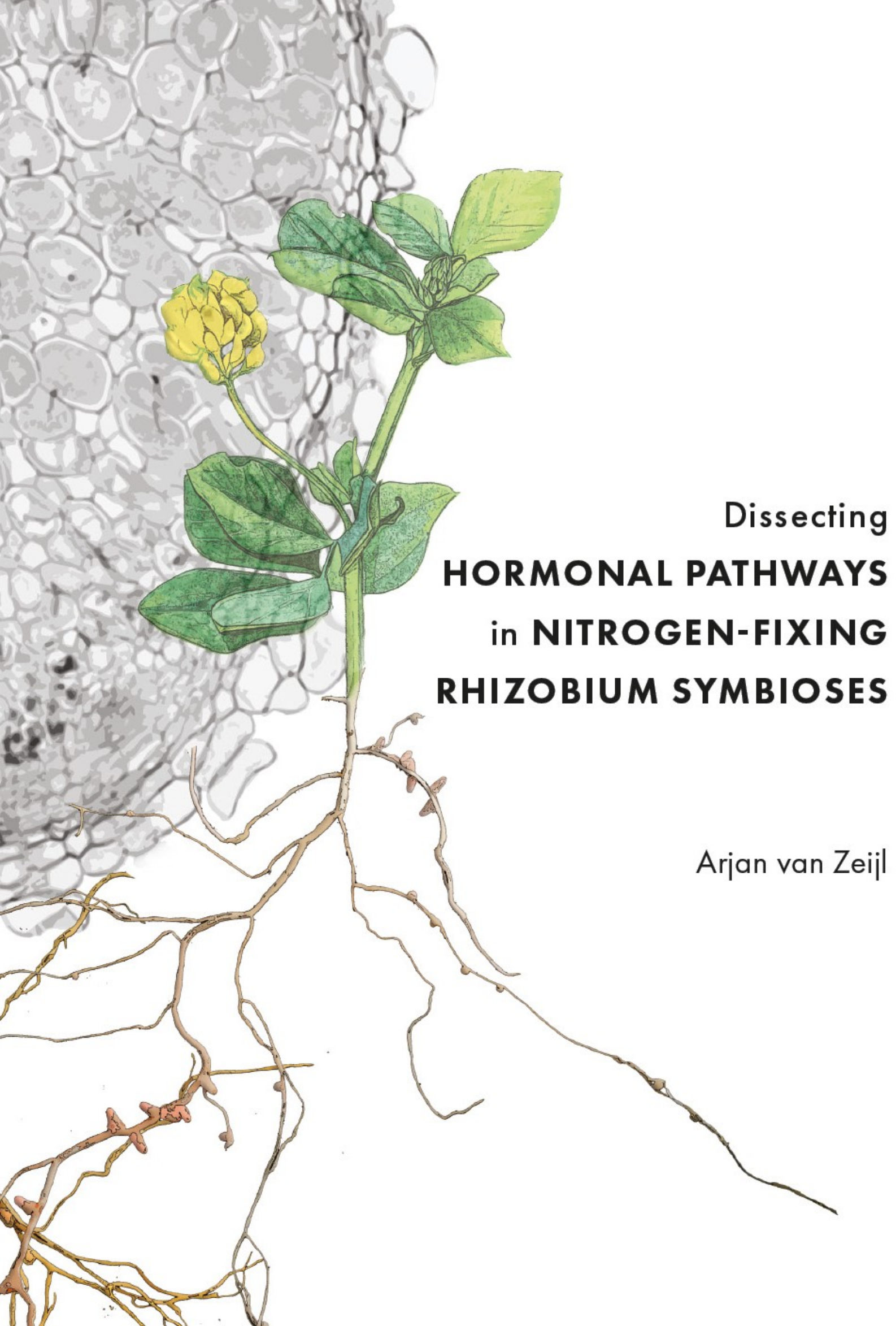


\section{Propositions}

1. Gene expression studies are poor predictors of whether a gene commits an important function.

(this thesis)

2. Pleiotropic effects hinder detailed functional analysis of hormonal pathways in plant development.

(this thesis)

3. The need of 473 genes to produce a minimal synthetic Mycoplasma genitalium genome, twice that of the common core genes of Haemophilus influenza and $M$. genitalium, shows that 'the' minimal genome does not exist (Hutchison III et al., 2016, Science 351:aad6253).

4. Scientific breakthroughs, like CRISPR/Cas9 genome engineering, demonstrate the importance of unbiased fundamental science (Lander, 2016, Cell 164(1):18-28).

5. The strive for longevity of human life is in conflict with efforts to mitigate climate change.

6. The stories written by Indonesian students in the 'typical Dutch' section of 'Resource' demonstrate that, despite a shared history, Dutch culture is difficult to understand.

Propositions belonging to the thesis, entitled:

"Dissecting hormonal pathways in nitrogen-fixing rhizobium symbioses"

Arjan van Zeijl

Wageningen, 18 October 2017 


\section{Dissecting hormonal pathways in nitrogen-fixing rhizobium symbioses}

Arjan van Zeijl 


\section{Thesis committee}

\section{Promotor}

Prof. Dr T. Bisseling

Professor of Molecular Biology

Wageningen University \& Research

\section{Co-promotor}

Dr R. Geurts

Associate professor, Laboratory of Molecular Biology

Wageningen University \& Research

\section{Other members}

Prof. Dr D. Weijers, Wageningen University \& Research

Prof. Dr G.E.D. Oldroyd, John Innes Centre, Norwich, UK

Dr J.F. Arrighi, IRD, UMR LSTM, Montpellier, France

Dr M.H.A.J. Joosten, Wageningen University \& Research

This research was conducted under the auspices of the Graduate School Experimental Plant Sciences 


\title{
Dissecting hormonal pathways in nitrogen-fixing rhizobium symbioses
}

\author{
Arjan van Zeijl
}

Thesis

submitted in fulfilment of the requirements for the degree of doctor at Wageningen University

by the authority of the Rector Magnificus,

Prof. Dr A.P.J. Mol,

in the presence of the

Thesis Committee appointed by the Academic Board

to be defended in public

on Wednesday 18 October 2017

at 1.30 p.m. in the Aula. 
Arjan van Zeijl

Dissecting hormonal pathways in nitrogen-fixing rhizobium symbioses, 232 pages.

PhD thesis, Wageningen University, Wageningen, the Netherlands (2017) With references, with summaries in Dutch and English

ISBN: 978-94-6343-631-1

DOI: $10.18174 / 419674$ 


\section{TABLE OF CONTENTS}

$\begin{array}{lll}\text { CHAPTER } 1 & \text { General Introduction } & 7\end{array}$

CHAPTER 2 Lipochitooligosaccharides modulate plant host immunity to 31 enable endosymbioses

CHAPTER 3 Rhizobium lipochitooligosaccharide signaling triggers accumulation of cytokinins in Medicago truncatula roots

CHAPTER 4 The strigolactone biosynthesis gene DWARF27 is co-opted in rhizobium symbiosis

CHAPTER 5 CRISPR/Cas9-mediated mutagenesis of four putative symbiosis genes of the tropical tree Parasponia andersonii reveals novel phenotypes

CHAPTER 6 Nodulating Parasponia and non-nodulating Trema species harbor distinct root transcriptomes

CHAPTER 7 General Discussion: Recruitment of hormonal networks in nitrogen-fixing nodule symbioses

Summary

Samenvatting

Acknowledgements

List of publications

Curriculum vitae

Education statement 



\section{Chapter 1}

General Introduction 


\section{INTRODUCTION}

Nitrogen is a key nutrient for plant growth and development. However, in many ecosystems, availability of fixed forms of nitrogen, such as nitrates or ammonium, is limiting. To overcome nitrogen limitation, some plants evolved the ability to utilize atmospheric nitrogen through a nodular endosymbiosis with nitrogen-fixing soil bacteria $[1,2]$. In this interaction, the bacteria are intracellularly accommodated in a specialized organ formed on the root of the plant, named the nodule. Inside nodules, the bacteria convert atmospheric nitrogen into ammonium, which they provide to the plant in exchange for photosynthates.

A major research objective of the scientific community is to unravel the evolutionary trajectory towards such nitrogen-fixing endosymbioses. Understanding the genetic basis of such symbioses, provides insight in the processes that lead to the evolution of a major ecological trait. Additionally, it could provide tools to engineer nitrogen-fixation in important crop plants, e.g. rice $[3,4]$.

Nitrogen-fixing root nodule symbioses are restricted to a single clade of flowering plants, known as the nitrogen-fixation clade [5-7]. This clade consists of four orders, namely Fabales, Fagales, Cucurbitales and Rosales. Within the nitrogen-fixation clade, nodulating plant species can be found among ten separate lineages that are scattered among mostly non-nodulating plant species [8]. Among the different nodulating lineages, two types of associations can be distinguished based on the microbial symbiont. Legumes (Fabaceae) and Parasponia (Cannabaceae) are nodulated by different genera of gram negative bacteria belonging to the $\alpha-, \beta$ - and $\nu$-proteobacteria that are collectively called rhizobia [9]. Actinorhizal plants, representing eight lineages in the Fagales, Cucurbitales and Rosales orders, are nodulated by gram positive filamentous actinomycetes of the genus Frankia [10]. Based on the phylogenetic distribution of nodulating lineages within the nitrogenfixing clade, it was hypothesized that nodulation evolved multiple times in parallel [5-7]. Additionally, the fact that nodulation is restricted to a single clade suggested that at least the ancestral species were genetically predisposed to evolve nodulation [5-7]. An alternative explanation for the observed phylogenetic distribution is that nodulation evolved only once in the ancestor of the nitrogen-fixation clade and was subsequently lost in most of its descendants.

Several decades of research on nitrogen-fixing nodule symbioses resulted in two major findings. Firstly, it was found that legumes, Parasponia and actinorhizal plants use a conserved set of genes to establish a nitrogen-fixing nodule symbiosis [2]. Secondly, it was 
shown that several of these genes also function in a much older and far more widespread endosymbiosis; the symbiosis between plants and arbuscular mycorrhizal fungi [11-18]. These obligate biotrophic fungi colonize roots of most land plants and form highly branched hyphal networks inside existing root cortical cells, known as arbuscules $[19,20]$. This raises the question of how these existing pathways were rewired to support a nitrogen-fixing nodule symbiosis. Here, I will summarize current knowledge on symbiotic signaling and describe the strategy used in this thesis to provide novel insight in the evolution of nitrogenfixing nodule symbioses.

\section{Legume Root Nodule Formation and Intracellular Infection}

Most insight in the genetic basis of nitrogen-fixing symbioses has been obtained from studies on the interaction between rhizobium bacteria and a limited number of legume species. This showed that the legume-rhizobium interaction is initiated upon rhizospheric signal exchange between plant and microbe. In response to root exudates, containing flavonoids, rhizobium bacteria synthesize and secrete lipo-chitooligosaccharides (LCOs) [21]. These LCOs, also known as Nod factors, consist of four to five $ß-1,4$-linked $\mathrm{N}$-acetyl glucosamine residues coupled to a fatty acid chain [22] and are structurally related to LCOs produced by mycorrhizal fungi [23]. Rhizobium LCOs act as symbiotic signals that are perceived by specific receptors at the plant root epidermis [24-27]. LCO perception mitotically activates cells in the root cortex and in some species endodermis and pericycle $[28,29]$. This results in the formation of a nodule primordium of which some cells are susceptible to infection by rhizobium bacteria. However, to allow intracellular infection to occur, nodule organogenesis and bacterial entry into the root need to be tightly coordinated. Most legumes make use of a root hair-based infection mechanism to allow bacterial entry [30]. This infection mechanism is also driven by perception of rhizobium LCOs and involves a redirection of growth of the root hair cell that perceives the LCO signal [31]. This process, known as root hair curling, entraps the rhizobium bacteria inside an infection pocket. From these infection pockets, cell wall-bound infection threads are formed that guide the rhizobia to the newly formed nodule primordium. Once the nodule primordium is reached, the rhizobium bacteria are released into the host cell as transient nitrogen-fixing organelles. These so-called symbiosomes consist of 1-3 individual bacteria surrounded by a plant-derived membrane [32]. This membrane forms a symbiotic interphase for the transfer of ammonium from the bacteria to the host cell in exchange of photosynthates.

The signaling network involved in the perception of rhizobium LCOs has mainly been identified through genetic studies on the model legumes Medicago truncatula (medicago) and Lotus japonicus (lotus). Rhizobium LCOs are perceived by a heteromeric complex of 
plasma membrane-localized LysM-domain receptor kinases [24-27,33]. Rhizobium LCO signaling requires a genetic network that is largely shared with the mycorrhizal symbiosis. This network, also known as the common symbiosis signaling cascade, consists of an LRRtype receptor kinase (LjSYMRK/MtDMI2), an enzyme involved in mevalonate biosynthesis (MtHMGR1), components of the nuclear pore complex, a nuclear-localized calciumdependent adenosine triphosphatase (MtMCA8), a nuclear-localized potassium channel (LjCASTOR/MtDMI1, LjPOLLUX) and nuclear-localized calcium channels (MtCNGC15a-c) [11-13,34-36]. A key response activated by this signaling network is the induction of regular oscillations of the nuclear calcium concentration, a response known as calcium spiking [37]. This calcium signal is decoded by a calcium- and calmodulin-dependent protein kinase (CCaMK) [18]. CCaMK interacts with and phosphorylates the transcription factor LjCYCLOPS/ MtIPD3 $[14,38,39]$. Phosphorylation of CYCLOPS by CCaMK activates separate transcriptional cascades for either the mycorrhizal or rhizobium symbiosis. In case of rhizobium symbiosis, a network of closely interacting transcription factors is activated, which includes NSP1, NSP2, NF-YA1, ERN1, ERN2 and NIN [39-48]. The readout of this network is not very well understood but involves massive transcriptional reprogramming [49-51]. Ultimately, the readout of LCO signaling affects the fate of cells in the root cortex, resulting in mitotic activation and subsequent nodule formation [2].

\section{The Role of Plant Hormones in Legume Symbioses}

In plant development, lateral organ formation is commonly associated with the formation of a local auxin maximum [52]. Also during nodule formation, a local auxin response maximum is observed in the cells that form the nodule primordium [53-58]. Genetic studies, however, mainly point at an important role for cytokinin signaling in legume nodule formation $[59,60]$. These studies indicated that a specific cytokinin receptor is not only required but also sufficient to induce nodule organogenesis $[59,60]$. This receptor, named LjLHK1 in lotus and MtCRE1 in medicago, is orthologous to the AtAHK4/AtCRE1 cytokinin receptor from Arabidopsis thaliana (arabidopsis) [61-64]. Genetic analyses positioned this receptor downstream of CCaMK but upstream of NIN in the symbiosis signaling network $[59,65]$. Gain-of-function mutants of $L j L H K 1 / M T C R E 1$ can form nodules in the absence of rhizobium bacteria $[59,66]$. Similarly, exogenous application of cytokinin induces the formation of nodule-like structures on the roots of several legume plants [67-70]. On the other hand, loss-of-function mutants in LjLHK1/MtCRE1 develop only limited number of nodules and the formation of these nodules is severely delayed [60,61]. The formation of a few nodules in Ljlhk1/Mtcre1 mutants is probably due to redundancy, as nodulation is completely abolished in a triple mutant in lotus in which two additional cytokinin receptors have been mutated or after RNAi-mediated silencing of additional cytokinin receptors in the Mtcre1-1 
mutant $[62,64]$. A role for cytokinin signaling in nodule formation is also supported by the rapid transcriptional activation of several type-A response regulators $[61,63,71,72]$. These genes are negative regulators of cytokinin signaling and are among the first responding genes following cytokinin perception [73]. Furthermore, exogenous application of cytokinin induces the expression of several symbiotic genes, including the transcription factors NSP1, NSP2, ERN1 and NIN [61,69]. Analysis of the cytokinin responsive reporter TCS in lotus suggests that cytokinin signaling is first induced in the dividing cortical cells that form the nodule primordium [62]. This is consistent with the expression pattern of MtRR4 in medicago, which is expressed in inner cortical cells already at six hours after rhizobium inoculation $[61,74]$. However, in a recent study in medicago an improved version of the TCS reporter was used, named TCSn [75], which revealed an initial activation of cytokinin signaling in the epidermis and outer cortex at four hours post LCO exposure [51]. These authors also performed laser-capture microdissection coupled to RNAseq, which confirmed the activation of cytokinin signaling genes in the epidermis. This analysis also revealed that cytokinin biosynthesis genes are induced in epidermal cells following LCO exposure [51]. Consistently, I show in Chapter 3 that cytokinin concentrations are increased within three hours of LCO exposure [76].

Despite the important role of cytokinin in nodule organogenesis, the mechanism by which cytokinin controls this process is not well understood. Recent evidence suggests that one of the main functions of cytokinin signaling is the induction of NIN expression in the root cortex [74]. NIN encodes a transcription factor that by itself is required but also sufficient to induce root nodule formation $[40,44,46,74]$. NIN expression is first induced by CYCLOPS in the epidermis, where it is required for root hair-based infection [39,40,65,74]. Additionally, NIN function in the root epidermis appears required for the activation of cytokinin signaling in the root cortex. Inoculation of wild-type medicago plants with rhizobium bacteria induces MtRR4 expression in inner root cortical cells, a response not observed in the Mtnin mutant [74]. Epidermal expression of NIN is sufficient to induce the formation of nodulelike structures; however, this requires MtCRE1. In contrast, expression of NIN in the root cortex induces nodule-like structures independent of MtCRE1 [74]. This suggests that NIN and cytokinin might form a positive feedback loop, in which epidermal expression of NIN activates cytokinin signaling in the root cortex. In turn, cytokinin is required to induce cortical expression of NIN and subsequent nodule formation.

Cytokinin signaling also seems important for the regulation of auxin transport during nodule formation. Measurements of auxin transport in wild-type medicago showed that acropetal (towards the root tip) auxin transport is reduced just below the inoculation site at 24 hours 
post rhizobial inoculation $[61,77,78]$. In contrast, basipetal (from the root tip upwards) auxin transport was increased just above the inoculation site [78]. Both responses are dependent on cytokinin signaling, as they were absent in the Mtcre1 mutant $[61,78]$. A mathematical modeling study tested through which mechanism an auxin maximum could be created during root nodule symbiosis. This showed that a local reduction in auxin transport in the root cortex creates an auxin accumulation pattern that is most comparable to that observed during nodule formation [79]. How this fits with the auxin transport measurements is not completely clear at this point. However, the fact that application of auxin transport inhibitors to the Mtcre1 mutant rescues its nodulation phenotype, suggests that the regulation of auxin transport is an important function of cytokinin signaling during nodule initiation. Whether cytokinins regulate auxin transport directly, as shown in arabidopsis, or indirectly through for example flavonoids remains to be demonstrated [78,80-84].

In contrast to the positive role of auxin and cytokinin in nodule formation, the gaseous hormone ethylene negatively regulates rhizobial infection and nodule formation. Application of ethylene, or its biosynthetic precursor 1-amino-cyclopropane carboxylic acid (ACC), strongly inhibits nodule formation in several legume species [85-88]. On the other hand, application of the ethylene synthesis inhibitor aminoethoxyvinylglycine (AVG) promotes nodule formation and rhizobial infection [87-90]. Ethylene is perceived by a family of ERlocalized receptors. These receptors control activity of the EIN2 protein, a central component of the ethylene signaling pathway [91]. Perception of ethylene by the ethylene receptors results in cleavage and translocation of the C-terminal domain of EIN2 to the nucleus, where it activates downstream gene expression [91-93]. The Mtein2 mutant in medicago is hyperinfected by rhizobium and forms clusters of often fused nodule primordia $[29,86,94]$. Such nodule clusters are also observed on lotus plants in which the expression of both EIN2 orthologous copies is reduced through RNAi-mediated knockdown [95]. Mtein2 mutant nodules are radially distributed along the root, whereas in wild-type plants nodules often form opposite protoxylem poles [94]. This radial distribution might be regulated by local production of ethylene, as in pea (Pisum sativum) ethylene biosynthesis genes were shown to be expressed opposite phloem poles [96]. Ethylene affects nodule formation already at an early step in the LCO signaling cascade, probably upstream or at the point of calcium spiking. In the presence of ethylene, an increased concentration of LCO molecules is needed to induce calcium spiking [88]. Ethylene also affects the duration and frequency of calcium spiking, with a decrease of spiking frequency observed in the Mtein2 mutant [88]. Like the effect of ethylene, jasmonic acid and abscisic acid (ABA) have also been shown to negatively regulate calcium spiking $[97,98]$. Expression of a dominant-negative allele of arabidopsis Atabi1 in medicago roots induces ABA insensitivity and hyper-nodulation [98]. 
The GRAS-type transcriptional regulators NSP1 and NSP2 are essential for rhizobial infection and root nodule formation $[41,42,99]$. Both proteins are required for the transcriptional activation of several LCO-responsive genes, including NIN and ERN1 [100,101]. NSP1 can directly bind the promoters of these genes in vitro, however in planta this requires NSP2. NSP1 and NSP2 interact to form heterodimers and this interaction is required for root nodule formation [100]. NSP1 and NSP2 also promote mycorrhizal colonization, though are not essential [1,23,102-105]. Under non-symbiotic conditions, NSP1 and NSP2 are required to produce strigolactones [104]. In medicago nsp mutants as well as rice (Oryza sativa) lines in which both NSP1 and NSP2 are silenced, strigolactone levels are reduced and this correlates with reduced expression of D27 [104]. Preliminary binding studies suggest that NSP1 can bind to specific elements in the D27 promoter, thereby regulating its expression [104]. D27 encodes a carotenoid isomerase that commits the first enzymatic step in strigolactone biosynthesis, which is the conversion of all-trans-ß-carotene into 9-cis-ß-carotene [106]. Subsequent activity of the carotenoid cleavage dioxygenases CCD7 and CCD8 results in the formation of carlactone, a strigolactone precursor $[106,107]$. The conversion of carlactone into strigolactones involves a cytochrome P450 encoded by MAX1 [108-110]. Strigolactone biosynthesis and D27 expression is increased under nutrient limitation, especially phosphate starvation [111-113]. Under these conditions, part of the strigolactone pool is exuded into the rhizosphere to attract mycorrhizal fungi $[112,114,115]$. Strigolactones induce germination of mycorrhizal spores and stimulate hyphal branching, thereby, promoting plant root colonization $[116,117]$. Several lines of evidence suggest that strigolactones also promote root nodule formation. Exogenous application of a low concentration of the synthetic strigolactone analog GR24 promoted nodule formation in medicago and alfalfa (Medicago sativa) [118,119]. Furthermore, strigolactone-deficient mutants in pea (Pisum sativum) and lotus produce less nodules, a phenotype that can be rescued by external application of GR24 [120-122]. Additionally, expression of MtD27 and MtCCD8 is induced during rhizobium infection in medicago root hairs [49]. In Chapter 4, I show that symbiotic expression of MtD27 is dependent on NSP1-NSP2 and that MtD27 is expressed throughout nodule formation [123].

DELLA proteins are mostly known for their role as negative regulators of gibberellin (GA) signaling. GA perception by the receptor GID1 targets DELLA proteins for degradation by the $26 \mathrm{~S}$ proteasome [124]. DELLAs are emerging as transcriptional integrators of various signaling networks, due to their ability to interact with a range of different transcription factors, including those involved in jasmonic acid as well as ethylene signaling $[125,126]$. DELLAs also play a central role in rhizobium- as well as mycorrhizae-induced signaling [127131]. This is based on the finding that DELLAs can interact with CCaMK and CYCLOPS. This 
interaction stabilizes the CCaMK-CYCLOPS complex and results in increased phosphorylation of CYCLOPS by CCaMK $[129,130]$. Additionally, DELLAs were shown to interact with NSP2, NF-YA1 and RAD1, a GRAS-type transcription factor required for arbuscule formation $[127,130,132,133]$. This suggests that DELLAs might form a bridge between CCaMKCYCLOPS and other symbiotic transcription factors, including the NSP2-NSP1 heterodimer, NF-YA1 and RAD1. In medicago, MtDELLA1 was shown to bind to the promoter of MtERN1, which activates MtERN1 expression [127]. ERN1 encodes an AP2/ERF transcription factor that is involved in rhizobial infection and nodule formation $[45,48]$. Besides MtDELLA1, also MtNSP2-MtNSP1 and MtNF-YA1 were shown to bind the MtERN1 promoter and transactivate MtERN1 expression $[47,100]$. This suggests that the interaction between DELLAs, NSP2-NSP1 and NF-YA1 is relevant for the transcriptional regulation of at least MtERN1 but probably also other symbiotic genes. Consistent with the central position of DELLAs in symbiotic transcriptional networks, medicago mutants in either a single or multiple DELLA gene(s) are severely affected in rhizobial infection, nodule formation as well as arbuscule formation by mycorrhizal fungi $[127,128,130]$. Similarly, application of GA to roots hampers infection thread formation, nodule organogenesis and arbuscule formation [127-130]. This effect can be rescued by expression of a GA-resistant form of MtDELLA1, named della1- 18 [128]. In medicago, ectopic expression of della1- $\Delta 18$ is also sufficient to rescue arbuscule formation in the Mtcyclops mutant [128]. Consistently, ectopic expression in wild-type medicago induces the expression of multiple genes normally induced by infection with arbuscular mycorrhizal fungi [132,134]. Additionally, epidermal expression of della1- $\triangle 18$ in noninoculated medicago roots is sufficient to induce expression of MtERN1 and MtENOD11, a marker for rhizobial infection [127]. Several transcriptome studies indicate that the GA biosynthetic pathway is activated $12-48$ hours post rhizobial inoculation $[49,50,135,136]$. This suggests that GA might be produced following successful infection to attenuate LCOinduced signaling. Interestingly, several rhizobium bacteria are also found to produce GA and this reduces the number of nodules formed by the host plant [137]. It is proposed that rhizobium bacteria secrete GA to prevent infection by other bacteria [137].

Symbiotic susceptibility is affected by nutrient concentrations in the soil. In most legume species, nodule formation is suppressed when moderate concentrations of fixed nitrogen are available $[138,139]$. Experiments using exogenous application of nitrogen revealed that nitrogen affects symbiotic functioning at several levels [138]. This includes a reduction in the number of rhizobial infections, a decrease in nitrogen fixation per unit of nodule mass and a reduction in nodule size, such that in some cases nodule formation is completely blocked [138]. Several studies indicate that exogenous nitrogen also affects the induction of LCOresponsive genes, including NIN [140-142]. Mutants in the 'autoregulation of nodulation' 
pathway are unable to control nodule number and as a result are hyper-nodulated. These mutants, however, are also less sensitive to the effect of exogenous nitrate on nodulation $[143,144]$. Rhizobial inoculation as well as the addition of fixed nitrogen induces the expression of genes encoding small secreted peptides, known as CLE peptides [145-147]. These CLE peptides are transported upwards to the shoot, where they are perceived by a CLAVATA1-like receptor, named LjHAR1 in lotus and MtSUNN in medicago [148-150]. This results in the production of a signal that is transported to the roots to suppress root nodule formation. Analysis of lotus mutants affected in CLE-HAR1 signaling, suggested that this downwards signal could be cytokinin [151]. Ethylene has also been hypothesized to be involved in the negative regulation of nodule formation by fixed nitrogen. Increased rates of ethylene evolution have been measured after nitrate treatment of alfalfa as well as soybean (Glycine max) roots [152,153]. Additionally, application of the ethylene synthesis inhibitor AVG rescued nodule formation in nitrate-treated alfalfa roots [154]. In case of mycorrhization, mainly the concentration of inorganic phosphate is regulating the degree of colonization [155]. Interestingly, in arabidopsis, phosphate starvation reduces the concentration of GA in the root, resulting in DELLA stabilization [156]. This suggests that mycorrhizal colonization in response to soil phosphate concentration might be regulated, in part, through an effect on the GA-DELLA balance [128].

\section{Evolutionary Trajectory Towards a Nitrogen-Fixing Endosymbiosis}

In legumes, Parasponia and actinorhizas activation of the common symbiosis signaling cascade mitotically activates cells leading to root nodule formation [2]. This is different from the mycorrhizal symbiosis where arbuscules are formed in existing cortical cells. This implies that symbiotic signaling or its readout has been adapted in legumes, Parasponia and actinorhizas to support root nodule formation.

A role for the common symbiotic signaling cascade in Parasponia and actinorhizal nodule formation is based on reverse genetic studies. These studies showed that PanNFP1, CgSYMRK/DgSYMRK, CgCCaMK and CgNIN are essential for nodule formation in Parasponia andersonii, Casuarina glauca and Datisca glomerata [157-161]. Additionally, measurements of the nuclear calcium concentration showed that calcium spiking, a hallmark of symbiotic signaling, is induced in roots of $P$. andersonii and the actinorhizal plants Alnus glutinosa and C. glauca in response to rhizobial or Frankia symbiotic signals $[162,163]$.

In Parasponia and most legume species, the common symbiosis signaling network is activated by rhizobium LCOs $[160,164]$. The Frankia symbiotic signals that activate actinorhizal nodule formation are currently unknown. Analysis of the genomes of two basal Frankia strains 
revealed that these strains encode LCO biosynthesis genes $[165,166]$. These genes are expressed inside nodules of $D$. glomerata, suggesting a role for Frankia LCOs in symbiosis with their actinorhizal hosts $[165,166]$. However, in most Frankia genomes LCO biosynthesis genes cannot be found [167]. Instead, characterization of the signaling molecules produced by Frankia sp. strain $\mathrm{Ccl} 3$ - a symbiont of $C$. glauca - suggests that these are biochemically distinct from LCOs [163]. LCO-independent nodulation mechanisms have also been described for several legume species belonging to the genus Aeschynomene as well as an LCO receptor mutant in soybean [168-170]. In the latter case, nodulation is dependent on the injection of rhizobial effectors via the type III secretion system, whereas LCO-independent nodulation of Aeschynomene legumes does not require effector delivery [168,171]. Despite differences in microbial signals, these LCO-independent nodulation mechanisms also involve activation of the common symbiosis signaling pathway. This because loss of the most upstream component, the LRR-type receptor SYMRK, abolishes nodule formation on Aeschynomene evenia as well as C. glauca [158,172].

To evolve susceptibility to rhizobial or Frankia signals, nodulating lineages might have obtained specific receptors. In medicago and lotus, both the LCO receptors MtLYK3/LjNFR1 and MtNFP/LjNFR5 resulted from gene duplications $[160,173,174]$. In case of MtLYK3/ LjNFR1, evidence supports that this duplication predated the evolution of rhizobium symbiosis in the legume family [174], suggesting that these receptors specifically evolved to function in rhizobium symbiosis. In Parasponia, two close homologs of MtNFP/LjNFR5 are present, of which PanNFP2 appears most closely related $[160,175]$. Orthologues of PanNFP2 are absent from the genomes of closely related non-nodulating Trema species [175], suggesting that PanNFP2 specifically evolved to recognize rhizobial LCOs. In line with this hypothesis is that calcium spiking cannot be observed in roots of Trema tomentosa after addition of LCOs from the broad host-range strain Sinorhizobium fredii NGR234 [162]. However, specific LCO receptors might not be sufficient to confer LCO-responsiveness. Ectopic expression of MtNFP and MtLYK3 under their native medicago promoters in tomato (Solanum lycopersicum), tobacco (Nicotiana tabacum), poplar (Populus x canescens) and strawberry (Fragaria $\mathrm{x}$ ananassa) proved insufficient to induce symbiotic responses [176]. Therefore, besides specific receptors, nodulating lineages might contain physiological adaptations that confer symbiotic susceptibility. This might include changes in nutritional status or in the hormonal balance, e.g. in ethylene, jasmonate or abscisic acid signaling.

Among nodulating lineages, activation of the common symbiosis signaling cascade appears sufficient to induce the onset of root nodule formation. In lotus or medicago, ectopic expression of wild-type or dominant active alleles of the nod factor receptors or SYMRK, 
CCAMK, CYCLOPS, LjLHK1/MtCRE1 or NIN is sufficient to induce nodule-like structures in the absence of rhizobium bacteria $[39,46,59,66,74,177-179]$. These so-called spontaneous nodules were also observed on roots of the actinorhizal plants C. glauca and Discaria trinervis as well as on roots of $P$. andersonii after ectopic expression of CgCCaMK, DtCCaMK and MtCCaMK, respectively $[160,161]$. However, activation of the common symbiosis signaling pathway by itself appears insufficient to explain the nodulation phenotype of legumes, Parasponia or actinorhizas. Genes encoding components of this pathway have been found in the genomes of all land plants examined, except for those that have lost the mycorrhizal symbiosis $[180,181]$. Also for legume symbiotic genes not part of the common symbiosis signaling pathway, putative orthologues have been found in non-nodulating plant species $[182,183]$. Trans-complementation assays of legume symbiotic mutants with orthologues from species outside the nitrogen-fixation clade suggest that these genes do not contain specific adaptations. These studies were mainly done using genes from rice, which showed that OsSYMRK, OsCASTOR, OsPOLLUX, OsCCaMK, OsCYCLOPS, OsNSP1 and OsNSP2 encompass all necessary functions to support root nodule formation [38,159,184-186]. Additionally, transfer of the nodulation trait to non-nodulating species based on expression of legume symbiosis genes proved unsuccessful [176]. Therefore, legumes, Parasponia and actinorhizas most likely possess specific adaptations to the readout of LCO signaling that are essential for nodule organogenesis.

A possible adaptation to the readout of LCO signaling is the activation of cytokinin signaling. As discussed above, activation of cytokinin signaling in response to the perception of rhizobial LCOs is essential for root nodule formation in lotus and medicago [60,61]. In contrast, cytokinin signaling appears not to be required for mycorrhization [187]. This suggests that in legumes, the cytokinin signaling pathway was specifically recruited to support root nodule formation. Mutagenesis of PanHK4, the orthologue of MtCRE1/LjLHK1 in $P$. andersonii, showed that this gene is not essential for Parasponia nodule organogenesis (Chapter 5). However, it should be noted that redundant functioning of additional cytokinin receptors cannot be excluded. Therefore, a better understanding of cytokinin functioning during nodule formation is required to determine how cytokinin signaling was recruited to support nodule formation in legumes and potentially other nodulating lineages.

\section{A Strategy to Uncover Symbiotic Adaptations}

The main objective of this thesis was to identify evolutionary adaptations that underlie the emergence of a nitrogen-fixing root nodule symbiosis. One way to achieve this is to deepen our knowledge on the readout of rhizobium-induced signaling in legumes. Legumes are very powerful in this respect as many experimental tools are already available. To determine 
whether candidates that come from such studies might represent lineage-specific adaptations or are also required for nitrogen-fixing symbioses in other lineages, studies on Parasponia that represents an 'evolutionary replicate' of the symbiosis are essential. This already proved to be successful, with respect to the role of the common symbiosis signaling cascade [2]. Another way to gain insight is to perform comparative studies on closely related species that differ in their ability to form nitrogen-fixing root nodules. Such studies could include genome as well as transcriptome comparisons (see van Velzen et al. [175]; Chapter 6). The success of these comparisons highly depends on the genetic distance between species. In this respect, Parasponia could provide an ideal model system, as it is closely related to non-nodulating Trema (see below). Therefore, I propose to adopt Parasponia as an experimental model system, which could be used alongside legumes, to decipher the genetic basis of nitrogen-fixing root nodule symbioses.

\section{The Parasponia-Trema Experimental Model System}

Parasponia represents a phylogenetic clade consisting of five tropical tree species indigenous to the Malay Archipelago [188]. Phylogenetic reconstruction indicates that the Parasponia lineage is embedded in the non-nodulating genus Trema $[175,189]$. This close phylogenetic relationship is underlined by recent analysis of the genome sequences of several Parasponia and Trema species that showed that these species are genetically very similar [175]. In fact, these species are so closely related that in rare cases inter-specific crosses are possible [175]. This suggests that the Parasponia lineage, including its symbiotic capacity, only recently evolved from an ancestral Trema species [190]. However, a different scenario is suggested by evidence for recent parallel pseudogenization of orthologues of PanNFP2, PanNIN and PanRPG in Trema spp. [175]. These genes perform essential symbiotic functions in legumes $[24,26,40,191]$. Also in the genomes of other Rosales species evidence for pseudogenization of NFP2, NIN and RPG was found [175]. This suggests the possibility that Trema and related Rosales species once possessed the capacity to nodulate. Irrespective of which evolutionary scenario is correct, studies on Parasponia in comparison to legumes or non-nodulating Trema spp. can still provide valuable insights into the genetic architecture of nitrogen-fixing endosymbioses.

Research on the nitrogen-fixing symbiosis of Parasponia already resulted in the development of several experimental procedures that aid this research. Efficient protocols for in vitro propagation of P. andersonii, Parasponia rigida and T. tomentosa are available [192194]. Additionally, protocols for seed propagation and germination have been developed $[175,195]$. Also, reference quality genomes for $P$. andersonii and Trema orientalis have been generated as well as several draft genomes for additional Parasponia and Trema species 
[175]. These provide a platform for genome as well as transcriptome studies. However, to analyze candidate genes coming from such analyses, reverse genetic tools are essential. To this end, a protocol for transient root transformation of $P$. andersonii and $T$. tomentosa has been developed [193]. This protocol is based on the formation of transgenic roots after transfer of the root inducing locus (rol) by Agrobacterium rhizogenes. Unfortunately, this affects the root's hormonal balance [196] and is therefore not well suited for the analysis of genes involved in hormone homeostasis. Additionally, this system is based on transient transformation of roots meaning that material cannot be maintained and each root results from an individual transformation event, resulting in considerable variation. To overcome this, I developed a protocol for stable transformation of Parasponia. Using this protocol, stable transgenic lines can be obtained in a period of four months. In combination with CRISPR/Cas9 mutagenesis, stable knockout mutants can be generated. This makes Parasponia well-suited for reverse genetic analysis (Chapter 5). 


\section{REFERENCES}

1 Delaux, P.M. et al. (2015) Tracing the evolutionary path to nitrogen-fixing crops. Curr. Opin. Plant Biol. 26, 95-99

2 Geurts, R. et al. (2016) What does it take to evolve a nitrogen-fixing endosymbiosis? Trends Plant Sci. 21, 199-208

3 Charpentier, M. and Oldroyd, G. (2010) How close are we to nitrogen-fixing cereals? Curr. Opin. Plant Biol. 13, 556-564

4 Oldroyd, G.E.D. and Dixon, R. (2014) Biotechnological solutions to the nitrogen problem. Curr. Opin. Biotechnol. 26, 19-24

5 Doyle, J.J. (2011) Phylogenetic perspectives on the origins of nodulation. Mol. Plant-Microbe Interact. 24, 1289-1295

6 Werner, G.D.A. et al. (2014) A single evolutionary innovation drives the deep evolution of symbiotic $\mathrm{N}_{2}$ fixation in angiosperms. Nat. Commun. 5, 4087

7 Soltis, D.E. et al. (1995) Chloroplast gene sequence data suggest a single origin of the predisposition for symbiotic nitrogen fixation in angiosperms. Proc. Natl. Acad. Sci. U. S. A. 92, 2647-2651

8 Li, H.-L. et al. (2015) Large-scale phylogenetic analyses reveal multiple gains of actinorhizal nitrogen-fixing symbioses in angiosperms associated with climate change. Sci. Rep. 5, 14023

9 Remigi, P. et al. (2016) Symbiosis within symbiosis: evolving nitrogen-fixing legume symbionts. Trends Microbiol. 24, 63-75

10 Santi, C. et al. (2013) Biological nitrogen fixation in non-legume plants. Ann. Bot. 111, 743-767

11 Stracke, S. et al. (2002) A plant receptor-like kinase required for both bacterial and fungal symbiosis. Nature 417, 959-962

12 Ané, J.-M. et al. (2004) Medicago truncatula DMI1 required for bacterial and fungal symbioses in legumes. Science 303, 1364-1367

13 Imaizumi-Anraku, H. et al. (2005) Plastid proteins crucial for symbiotic fungal and bacterial entry into plant roots. Nature 433, 527-531

14 Horváth, B. et al. (2011) Medicago truncatula IPD3 is a member of the common symbiotic signaling pathway required for rhizobial and mycorrhizal symbioses. Mol. Plant-Microbe Interact. 24, 1345-1358

15 Kanamori, N. et al. (2006) A nucleoporin is required for induction of $\mathrm{Ca}^{2+}$ spiking in legume nodule development and essential for rhizobial and fungal symbiosis. Proc. Natl. Acad. Sci. U. S. A. 545, 359-364

16 Saito, K. et al. (2007) NUCLEOPORIN85 is required for calcium spiking, fungal and bacterial symbioses, and seed production in Lotus japonicus. Plant Cell 19, 610-624

17 Groth, M. et al. (2010) NENA, a Lotus japonicus homolog of Sec13, is required for rhizodermal infection by arbuscular mycorrhiza fungi and rhizobia but dispensable for cortical endosymbiotic development. Plant Cell 22, 2509-2526

18 Lévy, J. et al. (2004) A putative $\mathrm{Ca}^{2+}$ and calmodulin-dependent protein kinase required for bacterial and fungal symbioses. Science 303, 1361-1364

19 Martin, F.M. et al. (2017) Ancestral alliances: Plant mutualistic symbioses with fungi and bacteria. Science 356, eaad4501

20 Gutjahr, C. and Parniske, M. (2013) Cell and developmental biology of arbuscular mycorrhiza symbiosis. Annu. Rev. Cell Dev. Biol. 29, 593-617

21 Dénarié, J. et al. (1996) Rhizobium lipo-chitooligosaccharide nodulation factors: signaling molecules mediating recognition and morphogenesis. Annu. Rev. Biochem. 65, 503-535

22 Lerouge, P. et al. (1990) Symbiotic host-specificity of Rhizobium meliloti is determined by a sulphated and acylated glucosamine oligosaccharide signal. Nature 344, 781-784

23 Maillet, F. et al. (2011) Fungal lipochitooligosaccharide symbiotic signals in arbuscular mycorrhiza. Nature 469, 58-63

24 Madsen, E.B. et al. (2003) A receptor kinase gene of the LysM type is involved in legume perception of rhizobial signals. Nature 425, 637-640

25 Radutoiu, S. et al. (2003) Plant recognition of symbiotic bacteria requires two LysM receptor-like kinases. Nature 425, 585-592 
26 Arrighi, J.F. et al. (2006) The Medicago truncatula lysine motif-receptor-like kinase gene family includes NFP and new nodule-expressed genes. Plant Physiol. 142, 265-279

27 Limpens, E. et al. (2003) LysM domain receptor kinases regulating rhizobial Nod factor-induced infection. Science 302, 630-633

28 Timmers, A.C. et al. (1999) Refined analysis of early symbiotic steps of the Rhizobium-Medicago interaction in relationship with microtubular cytoskeleton rearrangements. Development 126, 3617-3628

29 Xiao, T.T. et al. (2014) Fate map of Medicago truncatula root nodules. Development 141, 3517-3528

30 Gage, D.J. (2004) Infection and invasion of roots by symbiotic, nitrogen-fixing rhizobia during nodulation of temperate legumes. Microbiol. Mol. Biol. Rev. 68, 280-300

31 Esseling, J.J. (2003) Nod Factor-induced root hair curling: continuous polar growth towards the point of Nod Factor application. Plant Physiol. 132, 1982-1988

32 Roth, L.E. and Stacey, G. (1989) Bacterium release into host cells of nitrogen-fixing soybean nodules: the symbiosome membrane comes from three sources. Eur. J. Cell Biol. 49, 13-23

33 Moling, S. et al. (2014) Nod Factor receptors form heteromeric complexes and are essential for intracellular infection in Medicago nodules. Plant Cell 26, 4188-4199

34 Endre, G. et al. (2002) A receptor kinase gene regulating symbiotic nodule development. Nature 417, 962 966

35 Charpentier, M. et al. (2016) Nuclear-localized cyclic nucleotide-gated channels mediate symbiotic calcium oscillations. Science 352, 1102-1105

36 Venkateshwaran, M. et al. (2015) A role for the mevalonate pathway in early plant symbiotic signaling. Proc. Natl. Acad. Sci. U. S. A. 112, 9781-9786

37 Oldroyd, G.E.D. (2013) Speak, friend, and enter: signalling systems that promote beneficial symbiotic associations in plants. Nat. Rev. Microbiol. 11, 252-63

38 Yano, K. et al. (2008) CYCLOPS, a mediator of symbiotic intracellular accommodation. Proc. Natl. Acad. Sci. U. S. A. $105,20540-20545$

39 Singh, S. et al. (2014) CYCLOPS, a DNA-binding transcriptional activator, orchestrates symbiotic root nodule development. Cell Host Microbe 15, 139-152

40 Schauser, L. et al. (1999) A plant regulator controlling development of symbiotic root nodules. Nature 402 , 191-195

41 Kalo, P. et al. (2005) Nodulation signaling in legumes requires NSP2, a member of the GRAS family of transcriptional regulators. Science 308, 1786-1789

42 Smit, P. et al. (2005) NSP1 of the GRAS protein family is essential for rhizobial Nod factor-induced transcription. Science 308, 1789-1791

43 Andriankaja, A. et al. (2007) AP2-ERF transcription factors mediate Nod factor dependent Mt ENOD11 activation in root hairs via a novel cis-regulatory motif. Plant Cell 19, 2866-2885

44 Marsh, J.F. et al. (2007) Medicago truncatula NIN is essential for rhizobial-independent nodule organogenesis induced by autoactive calcium/calmodulin-dependent protein kinase. Plant Physiol. 144, 324-335

45 Middleton, P.H. et al. (2007) An ERF transcription factor in Medicago truncatula that is essential for Nod factor signal transduction. Plant Cell 19, 1221-1234

46 Soyano, T. et al. (2013) NODULE INCEPTION directly targets NF-Y subunit genes to regulate essential processes of root nodule development in Lotus japonicus. PLoS Genet. 9, e1003352

47 Laloum, T. et al. (2014) Two CCAAT-box-binding transcription factors redundantly regulate early steps of the legume-rhizobia endosymbiosis. Plant J. 79, 757-768

48 Cerri, M.R. et al. (2016) The symbiosis-related ERN transcription factors act in concert to coordinate rhizobial host root infection. Plant Physiol. 171, 1037-1054

49 Breakspear, A. et al. (2014) The root hair "infectome" of Medicago truncatula uncovers changes in cell cycle genes and reveals a requirement for auxin signaling in rhizobial infection. Plant Cell 26, 4680-4701

50 Larrainzar, E. et al. (2015) Deep sequencing of the Medicago truncatula root transcriptome reveals a massive and early interaction between Nod factor and ethylene signals. Plant Physiol. 169, 233-265

51 Jardinaud, M.-F. et al. (2016) A laser dissection-RNAseq analysis highlights the activation of cytokinin pathways by Nod factors in the Medicago truncatula root epidermis. Plant Physiol. 33, 2256-2276

52 Benkova, E. et al. (2003) Local, efflux-dependent auxin gradients as a common module for plant organ 
formation. Cell 115, 591-602

53 Takanashi, K. et al. (2011) Involvement of auxin distribution in root nodule development of Lotus japonicus. Planta 234, 73-81

54 van Noorden, G.E. et al. (2007) Overlap of proteome changes in Medicago truncatula in response to auxin and Sinorhizobium meliloti. Plant Physiol. 144, 1115-1131

55 Pacios-Bras, C. et al. (2003) Auxin distribution in Lotus japonicus during root nodule development. Plant Mol. Biol. 52, 1169-1180

56 Huo, X. et al. (2006) RNAi phenotypes and the localization of a protein::GUS fusion imply a role for Medicago truncatula PIN genes in nodulation. J. Plant Growth. Regul. 25, 156-165

57 Mathesius, U. et al. (1998) Auxin transport inhibition precedes root nodule formation in white clover roots and is regulated by flavonoids and derivatives of chitin oligosaccharides. Plant J. 14, 23-34

58 Suzaki, T. et al. (2012) Positive and negative regulation of cortical cell division during root nodule development in Lotus japonicus is accompanied by auxin response. Development 139, 3997-4006

59 Tirichine, L. et al. (2007) A gain-of-function mutation in a cytokinin receptor triggers spontaneous root nodule organogenesis. Science 315, 104-107

60 Murray, J.D. et al. (2007) A cytokinin perception mutant colonized by Rhizobium in the absence of nodule organogenesis. Science 315, 101-104

61 Plet, J. et al. (2011) MtCRE1-dependent cytokinin signaling integrates bacterial and plant cues to coordinate symbiotic nodule organogenesis in Medicago truncatula. Plant J. 65, 622-633

62 Held, M. et al. (2014) Lotus japonicus cytokinin receptors work partially redundantly to mediate nodule formation. Plant Cell 26, 678-694

63 Gonzalez-Rizzo, S. et al. (2006) The Medicago truncatula CRE1 cytokinin receptor regulates lateral root development and early symbiotic interaction with Sinorhizobium meliloti. Plant Cell 18, 2680-2693

64 Boivin, S. et al. (2016) Different cytokinin histidine kinase receptors regulate nodule initiation as well as later nodule developmental stages in Medicago truncatula. Plant, Cell Environ. 39, 2198-2209

65 Madsen, L.H. et al. (2010) The molecular network governing nodule organogenesis and infection in the model legume Lotus japonicus. Nat. Commun. 1, 10

66 Ovchinnikova, E. et al. (2011) IPD3 controls the formation of nitrogen-fixing symbiosomes in pea and Medicago spp. Mol. Plant-Microbe Interact. 24, 1333-1344

67 Torrey, J.G. (1961) Kinetin as trigger for mitosis in mature endomitotic plant cells. Exp. Cell Res. 23, 281-299

68 Mathesius, U. et al. (2000) Temporal and spatial order of events during the induction of cortical cell divisions in white clover by Rhizobium leguminosarum bv. trifolii inoculation or localized cytokinin addition. Mol. Plant-Microbe Interact. 13, 617-628

69 Heckmann, A.B. et al. (2011) Cytokinin induction of root nodule primordia in Lotus japonicus is regulated by a mechanism operating in the root cortex. Mol. Plant-Microbe Interact. 24, 1385-1395

70 Cooper, J. and Long, S. (1994) Morphogenetic rescue of Rhizobium meliloti nodulation mutants by transZeatin secretion. Plant Cell 6, 215-225

71 Vernié, T. et al. (2008) EFD is an ERF transcription factor involved in the control of nodule number and differentiation in Medicago truncatula. Plant Cell 20, 2696-2713

72 Op den Camp, R.H.M. et al. (2011) A phylogenetic strategy based on a legume-specific whole genome duplication yields symbiotic cytokinin Type-A Response Regulators. Plant Physiol. 157, 2013-2022

73 Hwang, I. et al. (2012) Cytokinin signaling networks. Annu. Rev. Plant Biol. 63, 353-380

74 Vernié, T. et al. (2015) The NIN transcription factor coordinates diverse nodulation programs in different tissues of the Medicago truncatula root. Plant Cell 27, 3410-3424

75 Zürcher, E. et al. (2013) A robust and sensitive synthetic sensor to monitor the transcriptional output of the cytokinin signaling network in planta. Plant Physiol. 161, 1066-1075

76 van Zeijl, A. et al. (2015) Rhizobium lipo-chitooligosaccharide signaling triggers accumulation of cytokinins in Medicago truncatula roots. Mol. Plant 8, 1213-1226

77 Wasson, A.P. et al. (2006) Silencing the flavonoid pathway in Medicago truncatula inhibits root nodule formation and prevents auxin transport regulation by rhizobia. Plant Cell 18, 1617-1629

$78 \mathrm{Ng}$, J.L.P. et al. (2015) Flavonoids and auxin transport inhibitors rescue symbiotic nodulation in the Medicago truncatula cytokinin perception mutant cre1. Plant Cell 27, 2210-2226

79 Deinum, E.E. et al. (2012) Modeling a cortical auxin maximum for nodulation: different signatures of 
potential strategies. Front. Plant Sci. 3, 96

80 Street, I.H. et al. (2016) Cytokinin acts through the auxin influx carrier AUX1 to regulate cell elongation in the root. Development 143, 3982-3993

81 Marhavy, P. et al. (2011) Cytokinin modulates endocytic trafficking of PIN1 auxin efflux carrier to control plant organogenesis. Dev. Cell 21, 796-804

82 Marhavy, P. et al. (2014) Cytokinin controls polarity of PIN1-dependent auxin transport during lateral root organogenesis. Curr. Biol. 24, 1031-1037

83 Laplaze, L. et al. (2007) Cytokinins act directly on lateral root founder cells to inhibit root initiation. Plant Cell 19, 3889-3900

84 Dello loio, R. et al. (2008) A genetic framework for the control of cell division and differentiation in the root meristem. Science 322, 1380-1384

85 Lee, K.H. and Larue, T. (1992) Exogenous ethylene inhibits nodulation of Pisum sativum L. cv Sparkle. Plant Physiol. 100, 1759-1763

86 Penmetsa, R.V. and Cook, D. (1997) A legume ethylene-insensitive mutant hyperinfected by its rhizobial symbiont. Science $275,527-530$

87 Nukui, N. et al. (2000) Effects of ethylene precursor and inhibitors for ethylene biosynthesis and perception on nodulation in Lotus japonicus and Macroptilium atropurpureum. Plant Cell Physiol. 41, 893-897

88 Oldroyd, G.E.D. et al. (2001) Ethylene inhibits the Nod factor signal transduction pathway of Medicago truncatula. Plant Cell 13, 1835-1849

89 Peters, N.K. and Crist-Estes, D.K. (1989) Nodule formation is stimulated by the ethylene inhibitor aminoethoxyvinylglycine. Plant Physiol. 91, 690-693

90 Guinel, F.C. and Larue, T.A. (1992) Ethylene inhibitors partly restore nodulation to pea mutant E107 (brz). Plant Physiol. 99, 515-518

91 Merchante, C. et al. (2013) Ethylene signaling: simple ligand, complex regulation. Curr. Opin. Plant Biol. 16, 554-560

92 Qiao, H. et al. (2012) Processing and subcellular trafficking of ER-tethered EIN2 control response to ethylene gas. Science 338, 390-393

93 Ju, C. et al. (2012) CTR1 phosphorylates the central regulator EIN2 to control ethylene hormone signaling from the ER membrane to the nucleus in Arabidopsis. Proc. Natl. Acad. Sci. U. S. A. 109, 19486-19491

94 Penmetsa, R.V. et al. (2008) The Medicago truncatula ortholog of Arabidopsis EIN2, sickle, is a negative regulator of symbiotic and pathogenic microbial associations. Plant J. 55, 580-595

95 Miyata, K. et al. (2013) Two distinct EIN2 genes cooperatively regulate ethylene signaling in Lotus japonicus. Plant Cell Physiol. 54, 1469-1477

96 Heidstra, R. et al. (1997) Ethylene provides positional information on cortical cell division but is not involved in Nod factor-induced root hair tip growth in Rhizobium-legume interaction. Development 124, 1781-1787

97 Sun, J. et al. (2006) Crosstalk between jasmonic acid, ethylene and Nod factor signaling allows integration of diverse inputs for regulation of nodulation. Plant J. 46, 961-970

98 Ding, Y. et al. (2008) Abscisic acid coordinates nod factor and cytokinin signaling during the regulation of nodulation in Medicago truncatula. Plant Cell 20, 2681-2695

99 Heckmann, A.B. et al. (2006) Lotus japonicus nodulation requires two GRAS domain regulators, one of which is functionally conserved in a non-legume. Plant Physiol. 142, 1739-1750

100 Hirsch, S. et al. (2009) GRAS proteins form a DNA binding complex to induce gene expression during nodulation signaling in Medicago truncatula. Plant Cell 21, 545-557

101 Mitra, R.M. et al. (2004) Six nonnodulating plant mutants defective for Nod factor-induced transcriptional changes associated with the legume-rhizobia symbiosis. Proc. Natl. Acad. Sci. U. S. A. 101, 10217-10222

102 Takeda, N. et al. (2013) CERBERUS and NSP1 of Lotus japonicus are common symbiosis genes that modulate arbuscular mycorrhiza development. Plant Cell Physiol. 54, 1711-1723

103 Shtark, O.Y. et al. (2016) Arbuscular mycorrhiza development in pea (Pisum sativum L.) mutants impaired in five early nodulation genes including putative orthologs of NSP1 and NSP2. Symbiosis 68, 129-144

104 Liu, W. et al. (2011) Strigolactone biosynthesis in Medicago truncatula and rice requires the symbiotic GRAS-type transcription factors NSP1 and NSP2. Plant Cell 23, 3853-3865

105 Lauressergues, D. et al. (2012) The microRNA miR171h modulates arbuscular mycorrhizal colonization of Medicago truncatula by targeting NSP2. Plant J. 72, 512-522 
106 Alder, A. et al. (2012) The path from $\beta$-carotene to carlactone, a strigolactone-like plant hormone. Science $335,1348-1351$

107 Seto, Y. et al. (2014) Carlactone is an endogenous biosynthetic precursor for strigolactones. Proc. Natl. Acad. Sci. U. S. A. 111, 1640-1645

108 Zhang, Y. et al. (2014) Rice cytochrome P450 MAX1 homologs catalyze distinct steps in strigolactone biosynthesis. Nat. Chem. Biol. 10, 1028-1033

109 Cardoso, C. et al. (2014) Natural variation of rice strigolactone biosynthesis is associated with the deletion of two MAX1 orthologs. Proc. Natl. Acad. Sci. U. S. A. 111, 2379-2384

110 Booker, J. et al. (2005) MAX1 encodes a cytochrome P450 family member that acts downstream of MAX3/4 to produce a carotenoid-derived branch-inhibiting hormone. Dev. Cell 8, 443-449

111 Yoneyama, K. et al. (2007) Phosphorus deficiency in red clover promotes exudation of orobanchol, the signal for mycorrhizal symbionts and germination stimulant for root parasites. Planta 225, 1031-1038

112 Yoneyama, K. et al. (2012) How do nitrogen and phosphorus deficiencies affect strigolactone production and exudation? Planta 235, 1197-1207

113 López-Ráez, J.A. et al. (2008) Tomato strigolactones are derived from carotenoids and their biosynthesis is promoted by phosphate starvation. New Phytol. 178, 863-874

114 Yoneyama, K. et al. (2007) Nitrogen deficiency as well as phosphorus deficiency in sorghum promotes the production and exudation of 5-deoxystrigol, the host recognition signal for arbuscular mycorrhizal fungi and root parasites. Planta 227, 125-132

115 Kretzschmar, T. et al. (2012) A petunia ABC protein controls strigolactone-dependent symbiotic signalling and branching. Nature 483, 341-344

116 Besserer, A. et al. (2006) Strigolactones stimulate arbuscular mycorrhizal fungi by activating mitochondria. PLoS Biol. 4, 1239-1247

117 Yoneyama, K. et al. (2008) Strigolactones, host recognition signals for root parasitic plants and arbuscular mycorrhizal fungi, from Fabaceae plants. New Phytol. 179, 484-494

118 De Cuyper, C. et al. (2014) From lateral root density to nodule number, the strigolactone analogue GR24 shapes the root architecture of Medicago truncatula. J. Exp. Bot. 66, 137-146

119 Soto, M.J. et al. (2010) First indications for the involvement of strigolactones on nodule formation in alfalfa (Medicago sativa). Soil Biol. Biochem. 42, 383-385

120 Foo, E. and Davies, N.W. (2011) Strigolactones promote nodulation in pea. Planta 234, 1073-1081

121 Foo, E. et al. (2013) Strigolactones and the regulation of pea symbioses in response to nitrate and phosphate deficiency. Mol. Plant 6, 76-87

122 Liu, J. et al. (2013) Carotenoid cleavage dioxygenase 7 modulates plant growth, reproduction, senescence, and determinate nodulation in the model legume Lotus japonicus. J. Exp. Bot. 64, 1967-1981

123 van Zeijl, A. et al. (2015) The strigolactone biosynthesis gene DWARF27 is co-opted in rhizobium symbiosis. BMC Plant Biol. 15, 260

124 Davière, J.-M. and Achard, P. (2013) Gibberellin signaling in plants. Development 140, 1147-1151

125 Davière, J.M. and Achard, P. (2016) A pivotal role of DELLAs in regulating multiple hormone signals. Mol. Plant 9, 10-20

126 Fonouni-Farde, C. et al. (2016) Root development and endosymbioses: DELLAs lead the orchestra. Trends Plant Sci. 21, 898-900

127 Fonouni-Farde, C. et al. (2016) DELLA-mediated gibberellin signalling regulates Nod factor signalling and rhizobial infection. Nat. Commun. 7, 12636

128 Floss, D.S. et al. (2013) DELLA proteins regulate arbuscule formation in arbuscular mycorrhizal symbiosis. Proc. Natl. Acad. Sci. U. S. A. 110, 5025-5034

129 Pimprikar, P. et al. (2016) A CCaMK-CYCLOPS-DELLA complex activates transcription of RAM1 to regulate arbuscule branching. Curr. Biol. 26, 987-998

130 Jin, Y. et al. (2016) DELLA proteins are common components of symbiotic rhizobial and mycorrhizal signalling pathways. Nat. Commun. 7, 12433

$131 \mathrm{Yu}$, N. et al. (2014) A DELLA protein complex controls the arbuscular mycorrhizal symbiosis in plants. Cell Res. 24, 130-133

132 Floss, D.S. et al. (2016) DELLA proteins regulate expression of a subset of AM symbiosis-induced genes in Medicago truncatula. Plant Signal. Behav. 2324, e1162369 
133 Xue, L. et al. (2015) Network of GRAS transcription factors involved in the control of arbuscule development in Lotus japonicus. Plant Physiol. 167, 854-871

134 Park, H.-J. et al. (2015) Hyphal branching during arbuscule development requires RAM1. Plant Physiol. 169, 2774-2788

135 Hayashi, S. et al. (2012) Transient Nod factor-dependent gene expression in the nodulation-competent zone of soybean (Glycine max [L.] Merr.) roots. Plant Biotechnol. J. 10, 995-1010

136 Libault, M. et al. (2010) Complete transcriptome of the soybean root hair cell, a single-cell model, and its alteration in response to Bradyrhizobium japonicum infection. Plant Physiol. 152, 541-552

137 Tatsukami, Y. and Ueda, M. (2016) Rhizobial gibberellin negatively regulates host nodule number. Sci. Rep. 6, 27998

138 Streeter, J. (1988) Inhibition of legume nodule formation and $\mathrm{N}_{2}$ fixation by nitrate. CRC. Crit. Rev. Plant Sci. 7, 1-23

139 Caetano-Anollés, G. and Gresshoff, P.M. (1991) Plant genetic control of nodulation. Annu. Rev. Microbiol. 45, 345-382

140 Barbulova, A. et al. (2007) Differential effects of combined N sources on early steps of the Nod factordependent transduction pathway in Lotus japonicus. Mol. Plant-Microbe Interact. 20, 994-1003

141 Heidstra, R. et al. (1997) Nod factor-induced expression of leghemoglobin to study the mechanism of $\mathrm{NH}_{4} \mathrm{NO}_{3}$ inhibition on root hair deformation. Mol. Plant-Microbe Interact. 10, 215-220

142 Nanjareddy, K. et al. (2014) Nitrate regulates rhizobial and mycorrhizal symbiosis in common bean (Phaseolus vulgaris L.). J. Integr. Plant Biol. 56, 281-298

143 Reid, D.E. et al. (2011) Molecular mechanisms controlling legume autoregulation of nodulation. Ann. Bot. 108, 789-795

144 Ferguson, B.J. et al. (2010) Molecular analysis of legume nodule development and autoregulation. J. Integr. Plant Biol. 52, 61-76

145 Reid, D.E. et al. (2011) Inoculation- and nitrate-induced CLE peptides of soybean control NARK-dependent nodule formation. Mol. Plant-Microbe Interact. 24, 606-618

146 Okamoto, S. et al. (2009) Nod factor/nitrate-induced CLE genes that drive HAR1-mediated systemic regulation of nodulation. Plant Cell Physiol. 50, 67-77

147 Mortier, V. et al. (2010) CLE peptides control Medicago truncatula nodulation locally and systemically. Plant Physiol. 153, 222-237

148 Krusell, L. et al. (2002) Shoot control of root development and nodulation is mediated by a receptor-like kinase. Nature 420, 422-426

149 Schnabel, E. et al. (2005) The Medicago truncatula SUNN gene encodes a CLV1-like leucine-rich repeat receptor kinase that regulates nodule number and root length. Plant Mol. Biol. 58, 809-822

150 Okamoto, S. et al. (2013) Root-derived CLE glycopeptides control nodulation by direct binding to HAR1 receptor kinase. Nat. Commun. 4, 2191

151 Sasaki, T. et al. (2014) Shoot-derived cytokinins systemically regulate root nodulation. Nat. Commun. 5, 4983

152 Ligero, F. et al. (1999) Nitrate- and inoculation-enhanced ethylene biosynthesis in soybean roots as a possible mediator of nodulation control. J. Plant Physiol. 154, 482-488

153 Ligero, F. et al. (1987) Evolution of ethylene from roots and nodulation rate of Alfalfa (Medicago sativa L.) plants inoculated with Rhizobium meliloti as affected by the presence of nitrate. J. Plant Physiol. 129, 461-467

154 Ligero, F. et al. (1991) Nitrate inhibition of nodulation can be overcome by the ethylene inhibitor aminoethoxyvinylglycine. Plant Physiol. 97, 1221-1225

155 Carbonnel, S. and Gutjahr, C. (2014) Control of arbuscular mycorrhiza development by nutrient signals. Front. Plant Sci. 5, 462

156 Jiang, C. et al. (2007) Phosphate starvation root architecture and anthocyanin accumulation responses are modulated by the gibberellin-DELLA signaling pathway in Arabidopsis. Plant Physiol. 145, 1460-1470

157 Clavijo, F. et al. (2015) The Casuarina NIN gene is transcriptionally activated throughout Frankia root infection as well as in response to bacterial diffusible signals. New Phytol. 208, 887-903

158 Gherbi, H. et al. (2008) SymRK defines a common genetic basis for plant root endosymbioses with arbuscular mycorrhiza fungi, rhizobia, and Frankia bacteria. Proc. Natl. Acad. Sci. U. S. A. 105, 4928-4932 
159 Markmann, K. et al. (2008) Functional adaptation of a plant receptor-kinase paved the way for the evolution of intracellular root symbioses with bacteria. PLoS Biol. 6, 497-506

160 Op den Camp, R. et al. (2011) LysM-type mycorrhizal receptor recruited for rhizobium symbiosis in nonlegume Parasponia. Science 331, 909-912

161 Svistoonoff, S. et al. (2013) The independent acquisition of plant root nitrogen-fixing symbiosis in fabids recruited the same genetic pathway for nodule organogenesis. PLoS One 8, e64515

162 Granqvist, E. et al. (2015) Bacterial-induced calcium oscillations are common to nitrogen-fixing associations of nodulating legumes and nonlegumes. New Phytol. 207, 551-558

163 Chabaud, M. et al. (2016) Chitinase-resistant hydrophilic symbiotic factors secreted by Frankia activate both $\mathrm{Ca}^{2+}$ spiking and NIN gene expression in the actinorhizal plant Casuarina glauca. New Phytol. 209, 86-93

164 Marvel, D.J. et al. (1987) Rhizobium symbiotic genes required for nodulation of legume and nonlegume hosts. Proc. Natl. Acad. Sci. U. S. A. 84, 1319-1323

165 Persson, T. et al. (2015) Candidatus Frankia Datiscae Dg1, the actinobacterial microsymbiont of Datisca glomerata, expresses the canonical nod genes nodABC in symbiosis with its host plant. PLoS One 10, e0127630

166 Nguyen, T.V. et al. (2016) An assemblage of Frankia Cluster II strains from California contains the canonical nod genes and also the sulfotransferase gene nodH. BMC Genomics 17, 796

167 Normand, P. et al. (2007) Genome characteristics of facultatively symbiotic Frankia sp. strains reflect host range and host plant biogeography. Genome Res. 17, 7-15

168 Okazaki, S. et al. (2013) Hijacking of leguminous nodulation signaling by the rhizobial type III secretion system. Proc. Natl. Acad. Sci. U. S. A. 110, 17131-17136

169 Giraud, E. et al. (2007) Legumes symbioses: absence of Nod genes in photosynthetic bradyrhizobia. Science 316, 1307-1312

170 Chaintreuil, C. et al. (2013) Evolution of symbiosis in the legume genus Aeschynomene. New Phytol. 200, 1247-1259

171 Okazaki, S. et al. (2016) Rhizobium-legume symbiosis in the absence of Nod factors: two possible scenarios with or without the T3SS. ISME J. 10, 64-74

172 Fabre, S. et al. (2015) The Nod factor-independent nodulation in Aeschynomene evenia required the common plant-microbe symbiotic "toolkit." Plant Physiol. 169, 2654-2664

173 Young, N.D. et al. (2011) The Medicago genome provides insight into the evolution of rhizobial symbioses. Nature 480, 520-524

174 De Mita, S. et al. (2014) Evolution of a symbiotic receptor through gene duplications in the legumerhizobium mutualism. New Phytol. 201, 961-972

175 van Velzen, R. et al. (2017) Parallel loss of symbiosis genes in relatives of nitrogen-fixing non-legume Parasponia. bioRxiv DOI: 10.1101/169706

176 Untergasser, A. et al. (2012) One-step Agrobacterium mediated transformation of eight genes essential for rhizobium symbiotic signaling using the novel binary vector system pHUGE. PLoS One 7, e47885

177 Tirichine, L. et al. (2006) Deregulation of a $\mathrm{Ca}^{2+} /$ calmodulin-dependent kinase leads to spontaneous nodule development. Nature 441, 1153-1156

178 Ried, M.K. et al. (2014) Spontaneous symbiotic reprogramming of plant roots triggered by receptor-like kinases. Elife 3, e03891

179 Gleason, C. et al. (2006) Nodulation independent of rhizobia induced by a calcium-activated kinase lacking autoinhibition. Nature 441, 1149-1152

180 Bravo, A. et al. (2016) Genes conserved for arbuscular mycorrhizal symbiosis identified through phylogenomics. Nat. Plants 2, 15208

181 Delaux, P.M. et al. (2014) Comparative phylogenomics uncovers the impact of symbiotic associations on host genome evolution. PLoS Genet. 10, e1004487

182 Yokota, K. and Hayashi, M. (2011) Function and evolution of nodulation genes in legumes. Cell. Mol. Life Sci. 68, 1341-1351

183 Soyano, T. and Hayashi, M. (2014) Transcriptional networks leading to symbiotic nodule organogenesis. Curr. Opin. Plant Biol. 20, 146-154

184 Banba, M. et al. (2008) Divergence of evolutionary ways among common sym genes: CASTOR and CCAMK show functional conservation between two symbiosis systems and constitute the root of a common 
signaling pathway. Plant Cell Physiol. 49, 1659-1671

185 Chen, C. et al. (2009) Antiquity and function of CASTOR and POLLUX, the twin ion channel-encoding genes key to the evolution of root symbioses in plants. Plant Physiol. 149, 306-317

186 Yokota, K. et al. (2010) Function of GRAS proteins in root nodule symbiosis is retained in homologs of a nonlegume, rice. Plant Cell Physiol. 51, 1436-1442

187 Laffont, C. et al. (2015) The CRE1 cytokinin pathway is differentially recruited depending on Medicago truncatula root environments and negatively regulates resistance to a pathogen. PLoS One 10, e0116819

188 Becking, J.H. (1992) The rhizobium symbiosis of the nonlegume Parasponia. In Biological Nitrogen Fixation (Stacey, G. et al., eds), pp. 497-559, Routledge, Chapman and Hall

189 Yang, M. et al. (2013) Molecular phylogenetics and character evolution of Cannabaceae. Taxon 62, 473-485

190 Behm, J.E. et al. (2014) Parasponia: a novel system for studying mutualism stability. Trends Plant Sci. 19, 757-763

191 Arrighi, J.-F. et al. (2008) The RPG gene of Medicago truncatula controls Rhizobium-directed polar growth during infection. Proc. Natl. Acad. Sci. U. S. A. 105, 9817-9822

192 Davey, M.R. et al. (1993) Effective nodulation of micro-propagated shoots of the non-legume Parasponia andersonii by Bradyrhizobium. J. Exp. Bot. 44, 863-867

193 Cao, Q. et al. (2012) Efficiency of Agrobacterium rhizogenes-mediated root transformation of Parasponia and Trema is temperature dependent. Plant Growth Regul. 68, 459-465

194 Webster, G. et al. (1995) The nodulation of micro-propagated plants of Parasponia andersonii by tropical legume rhizobia. J. Exp. Bot. 46, 1131-1137

195 Bender, G.L. and Rolfe, B.G. (1985) A rapid plant assay for the Parasponia-rhizobium symbiosis. Plant Sci. 38, 135-140

196 Nilsson, O. and Olsson, O. (1997) Getting to the root: The role of the Agrobacterium rhizogenes rol genes in the formation of hairy roots. Physiol. Plant. 100, 463-473 



\section{Chapter 2}

\section{Lipochitooligosaccharides modulate plant host immunity to enable endosymbioses}

Erik Limpens* , Arjan van Zeij|* and Rene Geurrs* (2015) Lipo-chitooligosaccharides modulate plant host immunity to enable endosymbioses. Annu. Rev. Phytopathol. 53: 311-334 *These authors contributed equally 


\section{Abstract}

Symbiotic nitrogen fixing rhizobium bacteria and arbuscular mycorrhizal fungi use lipochitooligosaccharide (LCO) signals to communicate with potential host plants. Upon a compatible match an intimate relation is established during which the microsymbiont is allowed to enter root(-derived) cells. Plants perceive microbial LCO molecules by specific LysM-domain containing receptor-like kinases. These do not only activate a 'common symbiosis signaling pathway' that is shared in both symbioses, but also modulate innate immune responses. Recent studies revealed that symbiotic LCO receptors are closely related to chitin innate immune receptors, and some of these receptors even function in symbiosis as well as immunity. This raises questions about how plants manage to translate structurally very similar microbial signals into different outputs. Here, we describe the current view on chitin and LCO perception in innate immunity and endosymbiosis and question how LCOs might modulate the immune system. Furthermore, we discuss what it takes to become an endosymbiont. 


\section{INTRODUCTION}

Plants are continuously exposed to microbes that range from beneficial to pathogenic. Plant roots, in particular, encounter massive numbers of microbes, known as the soil-root microbiome [1,2]. Two of the most extensively studied examples of beneficial microbes are arbuscular mycorrhizal (AM) fungi and nitrogen-fixing rhizobium bacteria. These microbes can live in intimate contact with their plant hosts and are intracellularly accommodated to establish an endosymbiotic relation. AM fungi are hosted inside the root inner cortex cells of the vast majority of land plants. Their highly branched hyphae are contained within specialized host membrane compartments to form host-microbe interface structures called arbuscules. The resulting symbiotic interface facilitates exchange of nutrients, such as phosphorus and nitrogen, for photosynthates [3]. Rhizobium bacteria establish an endosymbiosis with leguminous plants, which results in formation of a novel organ, the root nodule. Inside nodule cells, rhizobium bacteria are hosted as transient nitrogen-fixing organelles called symbiosomes. Symbiosomes contain a plant-derived outer membrane that forms a symbiotic interface between plant cyotplasm and bacterium facilitating exchange of nutrients between both partners. At the other end of the spectrum, pathogenic microbes can invade plant roots and extract nutrients at the expense of the plant. Biotrophic pathogens colonize living plant cells, where they form feeding structures, such as haustoria, that function as interfaces between plants and microbes, which are analogous to symbiotic interfaces.

Given the plethora of microbes that roots are exposed to, plants must be able to distinguish between friend and foe. Like pathogens, symbiotic microbes are initially recognized as foreign organisms, which triggers the plant innate immune system [4]. Microbes typically express a range of microbe/pathogen-associated molecular patterns (MAMPs/PAMPs) that are perceived by plant pattern recognition receptors to activate immune responses. This forms a first, basal level of defense to block microbial penetration of plant cells [57]. Therefore, endosymbionts like rhizobium and AM fungi have to modulate the innate immune system.

To establish symbiosis and evade innate immune responses, symbiotic microbes engage in a molecular dialog with potential plant hosts. Microbial lipochitooligosaccharides (LCOs) have been identified as the key signals to allow entry of the microbes into plant root cells. The use of LCOs appears to have been invented at least two times independently in evolution to establish an endosymbiosis with plants, first by AM fungi and later by rhizobium bacteria and possibly also by actinorhizal Frankia bacteria $[8,9]$. LCOs are structurally related to MAMPs 
such as chitin-oligosaccharides and peptidoglycans. In recent years, it has become clear that perception of all these chitin-like molecules involves related LysM-domain-containing receptor-like proteins. Furthermore, recent data indicate that perception of symbiotic LCOs is much more intertwined with innate immune signaling than previously imagined. This raises questions about how plants manage to integrate very similar microbial signals to distinguish friend from foe. Here, we review our current understanding of the perception of chitin-like molecules in endosymbioses and innate immune signaling and discuss how LCOs might modulate the immune system. Furthermore, we discuss what it takes to become an endosymbiont.

\section{The Ancient Arbuscular Mycorrhizal Symbiosis}

AM symbiosis between plants and fungi of the Glomeromycota phylum is thought to have originated $\sim 450$ million years ago in the Devonian era [10,11]. Fossil records from that period suggest that since then AM symbiosis has remained largely unchanged [12]. Fungal colonization of the root generally starts with formation of a hyphal hyphopodium at the epidermis. Subsequently, the plant actively facilitates the entry of fungal hyphae by formation of a pre-penetration apparatus through remodeling of its cytoskeleton [13]. After crossing the epidermis, fungal hyphae mostly spread intercellularly until they reach root inner cortex cells, where the hyphae branch and form intracellular arbuscules. During arbuscule formation, the hyphae become surrounded by a specialized host membrane to form an optimized symbiotic interface that facilitates the efficient exchange of nutrients [3]. This interface lacks a structured cell wall, which implies that extensive modulation of the host cell wall occurs [14]. Recently, unravelling of the genome sequence of the model AM fungus Rhizophagus irregularis revealed that this species lacks cell wall-degrading enzymes $[15,16]$. This indicates that AM fungi mostly rely on their plant hosts to deliver cell wall-remodeling enzymes to facilitate infection. Such enzymes might be delivered through a symbiosisdedicated exocytosis pathway. Two homologous v-SNARE proteins, part of a symbiotic clade, have been identified in the legume Medicago truncatula, which are essential for the formation of the cell wall-free interface in both AM and rhizobial symbioses [17]. Because fungal cell wall-degrading enzymes can release so-called damage-associated molecular patterns that trigger defense responses $[18,19]$, the absence of cell wall-degrading enzymes in AM fungi may therefore be a key adaptation to avoid a strong defense response.

\section{Signals in Arbuscular Mycorrhizal Symbiosis}

AM fungi sense the presence of potential host plants by plant secreted signals such as strigolactones, 2-hydroxy fatty acids, and flavonoids [20]. Perception of these molecules stimulates growth and branching of fungal hyphae, which increase the chance to contact 
the roots of a host plant. In turn, AM fungi release diffusible signals that are perceived by the host and induce symbiosis-specific responses. Typical responses include the induction of calcium oscillations in and around the nucleus (known as calcium spiking), stimulation of lateral root formation, activation of symbiosis-specific genes and branching of root hairs [21-24]. The nature of such AM signals was recently revealed by purifying exudate fractions of in vitro root cultures infected with $R$. irregularis and from germinated fungal spores $[25,26]$. By testing these fractions in various bioassays for symbiotic responses, it was found that $R$. irregularis makes acylated chitin-like molecules called myc-LCOs. MycLCOs consist of $\beta$-1-4-linked $N$-acetyl glucosamine (GlcNac) residues with an acyl chain at the non-reducing residue [26]. $R$. irregularis produces a mix of sulfated and non-sulfated tetrameric and pentameric LCOs mostly acylated with either an oleic acid (C18:1) or palmitic acid (C16:0) [26]. Application of such myc-LCO mixtures at sub-nanomolar concentrations stimulates mycorrhization in a variety of plant species. This indicates that myc-LCOs are efficiently perceived by a broad range of plants.

A key response that is triggered by myc-LCOs is the activation of calcium spiking, which forms a central response in a genetically identified signaling network. Initially, this network has been characterized in two legume species: $M$. truncatula and Lotus japonicus. Because the identified genes are essential for both AM and rhizobial symbioses, the genetic network is referred to as the common symbiosis signaling pathway [11,27]. This pathway consists of an LRR (leucine-rich repeat)-receptor kinase (named LjSYMRK in L. japonicus and MTDMI2 in M. truncatula) $[28,29]$, a putative cation channel located at the nuclear envelope (LjCASTOR, LjPOLLUX, and MtDMI1) [30-33], components of the nuclear pore [34-36], and a calcium channel (MtMCA8) [37]. These components are all required to induce calcium spiking $[38,39]$. These regular oscillations in calcium concentration induced upon LCO perception are decoded by a nuclear-localized calcium-calmodulin-dependent kinase (CCaMK; in M. truncatula also called MtDMI3) that interacts directly with the transcription factor LjCYCLOPS/MtIPD3 [40-42]. Phylogenetic studies revealed that the core components of the common symbiosis signaling pathway are conserved in the earliest land plants $[43,44]$. This is in line with the ancient origin of the AM symbiosis and the fact that AM fungi have an extremely wide host range.

In addition to LCOs, short-chain chitooligosaccharides consisting of four or five GICNAc residues that lack an acyl chain have been proposed to play a role in AM symbiosis [25]. Tetrameric and pentameric chitooligosaccharides were identified in germinated spore exudates at markedly higher concentration than LCOs and their secretion was induced by the application of strigolactones. Such short-chain chitooligosaccharides are also able to 
trigger calcium oscillations in epidermal cells of legume as well as non-legume (cultured) roots $[24,25]$. Like myc-LCO signaling, chitooligosaccharide-induced signaling is dependent on the common symbiosis signaling pathway. However, in contrast to myc-LCOs short-chain chitooligosaccharides fail to stimulate formation of lateral roots [26]. Further, the calcium signatures that are induced by chitin oligomers differ from the calcium spikes induced upon mycorrhizal (or rhizobial) infection or LCO application. Both mycorrhizal infection (cell entry) and LCO perception are correlated with high frequency and regular calcium spiking, whereas short-chain chitin oligomers induce irregular calcium spikes that are less frequent $[25,45]$. Low frequency calcium spiking has been correlated with pre-infection stages [45]. Therefore, the output of these calcium spikes may be different. These studies also indicate that perception of LCOs and chitin oligomers in root organ cultures differs markedly from that observed in intact plants. In contrast to intact plants, root organ cultures appear to be more than 1,000 times less sensitive to LCOs. This might be explained by an altered hormone balance in the root cultures. Therefore, it remains to be determined what role short-chain chitin oligomers play in mycorrhization in intact plants, and how they cross talk with myc-LCO signaling.

\section{Rhizobium Co-Opted Lipochitooligosaccharide Biosynthesis Through Convergent Evolution}

Myc-LCOs are structurally similar to LCOs produced by symbiotic nitrogen-fixing rhizobium bacteria (also known as Nod factors) $[26,46]$. Rhizobium bacteria secrete LCOs in response to a specific blend of amongst others flavonoids released by plant roots. Flavonoids activate the transcriptional activator NodD, which in turn induces rhizobial nodulation (nod) genes to produce LCOs [47]. Rhizobium LCOs control several key processes in symbiosis. They are essential, and even sufficient, to induce formation of root nodules. Nodule organogenesis starts with the re-programming of root cortical cells that re-enter the cell cycle to form a nodule primordium [48]. These primordium cells acquire the ability to take up rhizobium bacteria intracellularly. However, to ensure their uptake the bacteria need to reach the primordium cells at the right time. Therefore, the process of nodule organogenesis needs to be tightly coordinated with the entry of the bacteria into the roots, i.e., the infection process. This infection process is also controlled by rhizobium LCO signaling and can be genetically uncoupled from the organogenesis program [49]. Depending on the legume species, different modes of root infection have been identified [50]. The best studied and most common mechanism involves the formation of so-called infection threads that initiate in the root hairs [51]. Rhizobium bacteria attach to the root hairs and upon LCO signaling induce continuous reorientation of the growth direction of the root hair to entrap the bacteria in a closed cavity. From there, a cell wall-bound tube-like structure, the infection thread, is formed that guides the bacteria to the nodule primordium cells. At this point, the 
bacteria are first able to release from the infection threads to be taken up into the nodule cells and form symbiosomes. Also, this process depends on rhizobium LCO signaling [52]. The formation of infection threads in the root hairs is most sensitive to structural variations in the LCO molecules, which thereby play a major role in determining host specificity [5357].

Nitrogen-fixing rhizobium symbionts form a polyphyletic group representing 13 genera within the $\boldsymbol{\alpha}$-proteobacteria and 2 genera within the $\beta$-proteobacteria (Table 1) [58,59]. Symbiotic rhizobium bacteria have in common the fact that they combine two genetic traits, namely a set of nitrogen fixation (nif) genes that encode the nitrogenase enzyme complex and a set of (nod) genes that allow LCO biosynthesis. The nitrogen fixation trait has an ancient origin that dates back 1.5-2.2 billion years [60]. This trait was repeatedly horizontally transmitted to a diverse range of microbial species, including those that have given birth to current rhizobium symbionts [61,62]. An ancestral rhizobium symbiont managed, most probably as a unique evolutionary event, to combine the nitrogen fixation trait with its ability to produce LCOs. This event must have occurred at least 60 million years ago, prior to, or coinciding with, the birth of nitrogen fixing root nodules in legumes [63]. Once established, the unique combination of nitrogen fixation and LCO biosynthesis spread via lateral gene transfer [64]. Such spreading is especially prominent within the $\boldsymbol{\alpha}$-proteobacteria (Table 1 ). These rhizobium symbionts have organized the nitrogen fixation and LCO biosynthesis genes on symbiotic plasmids or as symbiotic islands in their genomes.

Symbiotic rhizobium species have in common a set of only five core nod genes, but generally contain many more lineage-specific genes that allow variation in LCO structure. The core nod genes encode an $\mathrm{N}$-acetylglucosaminyltransferase (NodC) that synthesizes the chitin tetramer or pentamer backbone, a chitooligosaccharide deacetylase (NodB) that removes the acetyl group of the non-reducing glucosamine, and an N-acyltransferase (NodA) that subsequently attaches a C16-C20 lipid tail to this position. Two additional core genes, nodl and nodJ, encode an ATP binding cassette $(A B C)$ transporter that facilitates LCO secretion [65-67]. As biosynthesis of chitin oligomers is not a common feature of prokaryotes, it raises questions about the evolutionary origin of these core nod genes. A survey of the $R$. irregularis genome sequence failed to identify homologs of these genes. Therefore, it is unlikely that an ancestral rhizobium acquired the ability to synthesize LCOs via lateral gene transfer from AM fungi. In search for paralogs of core nod genes, it was found that Burkolderia species ( $\beta$-proteobacteriales) harbor paralogs of nodl and nodJ. The genus Burkholderia is made up of many symbiotic rhizobia, and their symbiotic capacity is considered to be more than 50 million years old $[68,69]$. Interestingly, nodl-nodJ paralogous genes are absent in non- 
Table 1: Overview of symbiotic rhizobia in the orders Rhizobiales and Burkholderiales.

\begin{tabular}{|c|c|c|c|}
\hline \multicolumn{4}{|c|}{$\alpha$-Proteobacteria } \\
\hline Rhizobiales & Genera & $\begin{array}{c}\text { Number of } \\
\text { symbiotic genera }\end{array}$ & Symbiotic genera \\
\hline Aurantimonadaceae & 4 & - & \\
\hline Bartonellaceae & 1 & - & \\
\hline Beijerinckiaceae & 8 & - & \\
\hline Bradyrhizobiaceae & 12 & 2 & Bosea, Bradyrhizobium \\
\hline Brucellaceae & 6 & 1 & Ochrobactrum \\
\hline Cohaesibacteraceae & 2 & - & \\
\hline Hyphomicrobiaceae & 21 & 1 & Devosia \\
\hline Methylobacteriaceae & 3 & 2 & $\begin{array}{c}\text { Methylobacterium, } \\
\text { Microvirga }\end{array}$ \\
\hline Methylocystaceae & 8 & - & \\
\hline Phyllobacteriaceae & 12 & 3 & $\begin{array}{l}\text { Aminobacter, } \\
\text { Mesorhizobium, } \\
\text { Phyllobacterium }\end{array}$ \\
\hline Rhizobiaceae & 6 & 3 & $\begin{array}{c}\text { Rhizobium/ } \\
\text { Agrobacterium, Sinella, } \\
\text { Sinorhizobium }\end{array}$ \\
\hline Rhodobiaceae & 9 & - & \\
\hline Xanthobacteraceae & 7 & 1 & Azorhizobium \\
\hline \multicolumn{4}{|c|}{$\beta$-Proteobacteria } \\
\hline Burkholderiales & Genera & $\begin{array}{c}\text { Number of } \\
\text { symbiotic genera }\end{array}$ & Symbiotic genera \\
\hline Alcaligenaceae & 20 & - & \\
\hline Burkholderiaceae & 12 & 2 & Burkholderia, Cupriavidus \\
\hline Oxalobacteraceae & 15 & - & \\
\hline Sutterellaceae & 2 & - & \\
\hline
\end{tabular}

symbiotic $\boldsymbol{\alpha}$-proteobacteria, and phylogentic analyses suggest that at least the rhizobial LCO transport system originates from ancestral Burkholderia [70]. However, the origin of nodl and nodJ is not necessarily indicative for the origin of LCO biosynthesis in symbiotic rhizobia. Similar studies with $\operatorname{nod} A, \operatorname{nod} B$, and $\operatorname{nod} C$ did not identify putative paralogous genes, despite massive (meta) genome sequencing programs. Therefore, an alternative scenario in which LCO biosynthesis genes evolved first in a different, yet unknown, species cannot 
be ruled out. Only upon emergence of the nodABC operon in Burkholderia via lateral gene transfer were the nodl and nodJ genes recruited to facilitate LCO secretion. As symbiotic $\boldsymbol{\alpha}$-proteobacteria display greater sequence diversity in nodABC compared to Burkholderia, it is a likely scenario that this operon evolved first in an ancestral $\boldsymbol{\alpha}$-proteobacteria species $[69,70]$.

\section{Lipochitooligosaccharide Perception in Symbiosis Intertwines with Innate Immune Signaling}

Rhizobium and Myc-LCOs are structurally very similar and both are perceived by related LysM-domain-containing receptor-like kinases (LysM-RKs). The LysM (lysin motif) domain is a widely distributed protein domain that generally is thought to bind GIcNAc molecules [71]. Unique to plants is the presence of LysM domains in the extracellular part of transmembrane receptor kinases [72]. LysM-RKs are characterized by an extracellular part with one to three LysM domains that are distinct in sequence, suggesting a single evolutionary origin of this type of receptors [72].

Genetic and biochemical studies in L. japonicus and $M$. truncatula have led to the identification of two different LysM-RKs that are essential for rhizobium LCO perception and signaling: LjNFR1/MtLYK3 and LjNFR5/MtNFP [73-77]. Strikingly, LjNFR5 and MtNFP lack critical amino acids in the kinase domain and do not show autophosphorylation, indicating that they have a non-functional kinase $[73,76,78]$. However, deletion of the intracellular kinase domain abolishes the activity of MtNFP $[79,80]$, which suggests that specific binding partners confer the signaling role of these receptors. LjNFR5 and MtNFP have been shown to form a complex with LjNFR1 and MtLYK3, respectively, in addition to both proteins forming homomers $[52,78]$. LjNFR1 and MtLYK3 have a functional kinase domain, and heteromerization with LjNFR5/MtNFP is therefore proposed to constitute a functional rhizobium LCO receptor complex [78,80]. In addition, LjNFR5 was found to interact with LjSYMRK as well as with a small Rho-like GTPase LjROP6 [81,82]. LjSYMRK is cleaved in vivo, releasing a large part of its extracellular domain, allowing the remaining protein to interact specifically with LjNFR5 [82]. Both proteins, LjSYMRK/MtDMI2 and LjROP6, possibly in conjunction with LjNFR5/MtNFP, play a role in rhizobial infection.

Interestingly, myc-LCOs are also perceived by MtNFP, as the ability of myc-LCOs to induce lateral root formation in M. truncatula is dependent on MtNFP [26]. Furthermore, early transcriptional changes induced by myc-LCOs are largely dependent on MtNFP [83]. Rhizobium and myc-LCOs each trigger their own set of transcriptional changes, and differences were even observed between sulfated and non-sulfated myc-LCOs. This suggests that additional 
(co)receptors are involved. The involvement of additional myc-LCO (co)receptors is also supported by the fact that Ljnfr5/Mtnfp knockout mutants are not impaired in mycorrhizal colonization [75,84]. In M. truncatula, a paralog of MtNFP, MtLYR1, is upregulated upon mycorrhization [85-87]. Therefore, it has been proposed that duplication of an ancient LjNFR5/MtNFP-like myc-LCO receptor allowed one copy to obtain a new function in Nod factor signaling while the other maintained its role in mycorrhization [88]. This hypothesis is supported by the observation that in Parasponia, the only non-legume species able to establish a rhizobium symbiosis, only a single LjNFR5/MtNFP ortholog is present and is required for the intracellular accommodation of both rhizobia and AM fungi [88]. As Parasponia obtained the ability to nodulate relatively recently and independently from legumes [89], this strongly suggests that perception of rhizobium LCOs evolved from the ancient myc-LCO perception mechanism [8]. A recent origin of nodulation in Parasponia also fits with the primitive nature of rhizobium infection of this species. Whereas most legumes guide their rhizobial symbionts via intracellular infection threads toward the newly formed root nodule, Parasponia allow rhizobium to enter first apoplastically via a mechanism known as crack entry [90]. Only when it reaches the nodule is rhizobium hosted intracellularly. However, it is not hosted as a symbiosome but rather in so-called fixation threads that have some resemblance to mycorrhizal arbuscules. Knockdown of the Parasponia andersonii NFP ortholog (PaNFP) specifically blocks the formation of fixation threads as well as arbuscules, suggesting that this process may in fact be the generic function of symbiotic LCO signaling. In legumes, this mechanism evolved to allow more strict control over rhizobium infection and in some legumes co-evolved into a highly specific plant-rhizobium partner selection mechanism [56]. Whether MtLYR1 is indeed involved in myc-LCO perception remains to be demonstrated, but additional LCO (co)receptors may also be involved. Recently, it was shown that MtLYR3 has a high affinity for LCOs and is expressed in roots and nodules [91]. MtLYR3 and MtNFP are the result of an ancient tandem duplication, and their genomic location also shows synteny with the MtLYR1 genomic region [76]. Therefore, MtLYR3 may represent an additional coreceptor for LCO perception.

Analogous to LjNFR5/NFP-type LysM-RKs, it was found that LjNFR1 and MtLYK3 also play a role in mycorrhization. Mtlyk3 and Ljnfr1 mutants are impaired in mycorrhizal infection [92]. However, infection is not completely blocked, again suggesting that additional LysMRKs are involved. These data further suggest evolution of the rhizobium LCO perception mechanism from the ancient mycorrhizal signaling machinery. Intriguingly, it was found in rice (Oryza sativa) that a single receptor, OsCERK1, facilitates infection of AM fungi but is also essential for chitin-triggered defense responses [92,93]. OsCERK1 is a close homolog, if not an ortholog, of the chitin innate immune receptor AtCERK1 of Arabidopsis thaliana 
(Arabidopsis) and the legume rhizobium LCO receptors LjNFR1 and MtLYK3. The ability of LjNFR1/MtLYK3 and OSCERK1 to function in symbiotic signaling correlates with the presence of a YAQ/YAR motif in the kinase domain [93-95]. Defense-related chitin signaling does not require this motif, as Arabidopsis AtCERK1 appears to have lost it [94,95]. Furthermore, the kinase domain of OSCERK1 when fused to the extracellular part of LjNFR1 was able to fully complement the nodulation defect of the Ljnfr1 mutant [93]. This indicates that no additional evolution of the kinase domain is required to function in nodulation and underlines that symbiotic LCO perception and innate immune signaling intertwine.

\section{Chitin-Induced Innate Immune Signaling Shows Analogies to Symbiotic Lipochitooligo- saccharide Signaling}

In contrast to tetrameric and pentameric chitin oligomers, longer chain chitin oligomers (hexa- to octamers) or peptidoglycans are typically perceived as MAMPs that trigger innate immunity $[96,97]$. Perception of these chitin-like molecules also involves LysM-RKs. The functioning of these receptors has been best studied in Arabidopsis, a species that lost the AM symbiosis, and in rice, which does form an AM symbiosis.

In Arabidopsis, at least three LysM-RKs are involved in chitin-induced innate immune signaling: AtCERK1, AtLYK4, and AtLYK5 [98-104]. As mentioned above, AtCERK1 is a close homolog, or even an ortholog, of LjNFR1/MtLYK3 and was originally identified as the key chitin receptor on the basis of its mutant phenotype and its ability to bind chitin oligomers. Octameric chitin oligomers cause dimerization of AtCERK1, leading to activation of its kinase domain [105]. Short-chain chitin oligomers also bind to the extracellular part of AtCERK1 but fail to induce dimerization. Interestingly, such short-chain chitin oligomers could attenuate AtCERK1 signaling in a dose-dependent manner [105]. Therefore, it is possible that the short-chain chitin oligomers found in the exudates of AM fungi [25] actually interfere with defense activation by longer chain oligomers. The relative abundance of the different chitin oligomers may in such cases play an important role in determining the outcome of the responses. AtCERK1 can form a heteromer complex with AtLYK5 and AtLYK4 $[100,103]$. AtLYK5 and AtLYK4 belong to the same LysM-RK subfamily as LjNFR5/MtNFP and likewise do not have an active kinase domain. AtLYK5 binds chitin oligomers with much higher affinity than AtCERK1, indicating that AtLYK5 is in fact the major chitin receptor in Arabidopsis. AtLYK5 functions partly redundantly with AtLYK4, as both bind chitin and only a double mutant completely blocks chitin signaling, similar to Atcerk1 mutants. Binding of chitin oligomers by AtLYK5 induces heteromerization with AtCERK1, resulting in activation of its kinase domain [103]. This indicates the involvement of a receptor complex analogous to the LjNFR5-LjNFR1 and MtNFP-MtLYK3 receptor complexes in symbiosis. 
In contrast to AtCERK1, OsCERK1 was not found to bind chitin oligomers, indicating differences in affinity for chitin-like molecules $[102,106]$. To perceive myc-LCOs, it is likely that OsCERK1 pairs with LysM coreceptors, for which homologs of LjNFR5/MtNFP in rice, such as OsLYR1, are key candidates. To perceive chitooligosaccharides, rice relies on the LysM-domain-containing CHITIN ELICITOR BINDING PROTEIN (OsCEBiP), which lacks an intracellular kinase domain [106]. OsCEBiP binds chitin and forms a heteromer complex with OsCERK1 to activate chitin-triggered defense responses $[107,108]$. Notably, homologs of OsCEBiP in Arabidopsis, AtLYM1, AtLYM2, and AtLYM3 are not involved in AtCERK1mediated innate immunity $[100,102]$. Nevertheless, AtLYM2 was shown to bind chitin and contributed to resistance against the fungal pathogen Alternaria brassicicola in an AtCERK1-independent manner [101,109]. Additionally, AtLYM2 was shown to control the transport ability of plasmodesmata in a chitin-dependent manner, which plays a role in plant immunity to pathogenic fungi such as Botrytis cinerea [110]. This suggests that at least two different pathways (AtCERK1 dependent and AtCERK1 independent) are operational in chitin-based defense. It is currently not known with which coreceptor(s) AtLYM2 interacts to relay signaling. In contrast, AtLYM1 and AtLYM3 were shown to form a complex with AtCERK1 to trigger immunity in response to peptidoglycans [111]. In rice, the LYM homologs OsLYP4 and OsLYP6 play a role in peptidoglycan perception as well as chitin perception [105]. Overall, these data indicate that CERK1 homologs function as a coreceptor in diverse signaling complexes related to perception of chitin-like molecules, including LCOs.

\section{Two Models for Dual Functioning of Lipochitooligosaccharide LysM-RKs}

Specific gene duplication events early in legume evolution provided the freedom to evolve symbiosis specific functions of LjNFR1/MtLYK3 and LjNFR5/MtNFP $[72,88,95]$. However, three lines of evidence also indicate that LjNFR1/MtLYK3 and LjNFR5/MtNFP still have a dual function in symbiosis and defense signaling, in analogy to the dual function of OsCERK1. (I.) Rhizobium LCOs transiently trigger defense-related gene expression in an LjNFR1-dependent manner [94]. (II.) MtNFP acts, independent of MtLYK3, in defense against fungal pathogens, including Verticillium alboatrum and Colletotrichum trifolii, or the oomycete Aphanomyces euteiches [112,113]. A lack of MtNFP makes plants more susceptible to such pathogens, whereas ectopic expression of MtNFP increases resistance [112]. (III.) A strong coexpression of both receptors -LjNFR5-LjNFR1 or MtNFP-MtLYK3- in Nicotiana benthamiana leaves triggered a spontaneous cell death response $[77,80]$. This response requires an active LjNFR1/MtLYK3 kinase domain. An early cell death response was also observed in $M$. truncatula nodules when MtNFP was overexpressed [52]. This work further showed that both receptors are under tight posttranslational control in legume roots and nodules, likely to prevent premature induction of defense responses. Taken together, these data suggest 
that dual functioning of LCO receptors in defense and symbiosis is not unique for rice (and other non-legumes) but has also been maintained in legumes to function in rhizobium symbiosis. This implies that genetic constraints preserve this dual functioning [93]. The biological meaning of this dual functioning can be explained in two ways. A first model is based on competition for CERK1/NFR1/LYK3-type proteins by different receptor complexes. High affinity for LCOs (and/or short-chain chitin oligomers) leads to the preferential formation of symbiotic receptor complexes at the expense of the formation of complexes that act in defense signaling [93] (Figure 1A). Variations in affinities for different chitin-like molecules encoded by the extracellular receptor domains determine which complexes are formed. This would automatically block chitin-induced defense responses, without the need to independently suppress chitin-triggered immunity. This model is in line with the finding that LCOs can repress innate immunity [114]. As an alternative model, the MAMP-triggered responses may actually be recruited to facilitate/regulate the infection process by symbiotic microbes (Figure 1B). For example, several typical early MAMP-triggered responses, such as calcium influx, production of reactive oxygen species (ROS), and focal exocytosis, have been implicated in the formation and growth of infection threads during the rhizobium interaction [115]. Infection threads are tip-growing structures, and ROS production is thought to facilitate the oxidative cross-linking of the infection thread matrix to allow the formation of a tube-like infection thread. The spatiotemporal regulation of receptor complexes may then be important to fine-tune the responses to prevent too-strong defense responses [52]. This hypothesis is also in line with the essential role for symbiotic LCO receptors in infection thread formation $[53,54,57,74,76]$, which is independent from their role in activating the common symbiosis signaling pathway [116]. Several studies suggest that symbiotic LCO receptors are located in lipid-raft-like membrane domains in the plasma membrane, which is thought to play an important role in controlling their turnover and their ability to form specific complexes [117-119]. Localization of MtNFP and MtLYK3 in the nodule showed that these receptors accumulate in a very narrow zone at the nodule apex and are rapidly removed from the infection thread membrane to allow the release of the bacteria from the infection threads [52].

\section{Lipochitooligosaccharide Perception Is Not Sufficient to Evade Innate Immunity}

LCO perception may play an even broader role in suppression of PAMP-triggered immune responses, as LCOs were shown to suppress flg22 (a 22 amino acid peptide fragment of flagellin)-induced immune responses in various plant species. In Arabidopsis this response depends specifically on AtLYK3 but not on AtCERK1 [114]. Atlyk3 mutants appear to be more resistant to fungal pathogens such as $B$. cinerea and Pectobacterium carotovorum, suggesting that these pathogens exploit this receptor kinase to infect plants [120]. These 
data led to the speculation that suppression of innate immunity may have been the original/ first function of LCOs [121]. However, it is difficult to imagine why non-symbiotic plants such as Arabidopsis would have maintained a LCO perception system to repress innate immunity. It is also difficult to reconcile with the observation that LCOs do trigger defense responses [94]. Clearly, a better understanding of the cross talk between the various receptor complexes, potentially via shared downstream partners, involved in LCO and MAMP perception is required to resolve this apparent paradox.

Hijacking the LysM-RK activated common symbiosis signaling pathway, which is operational in the vast majority of land plants, would seem to be an attractive strategy for biotrophic pathogens to colonize root cells $[122,123]$. However, studies that indicate that pathogens make use of this strategy are exceptionally scarce. Root knot nematodes have been suggested to secrete signal molecules, which trigger similar root hair deformations as LCOs [124]. This ability is (partially) dependent on LjNFR1-LjNFR5 and LjSYMRK, and correspondently absent in root hairs of Arabidopsis. This led to the suggestion that root knot nematodes may produce LCO-like molecules to make use of the common symbiosis signaling pathway [124]. However, a clear involvement of the common symbiosis signaling pathway in root knot nematode infection has not been shown, and the nature of the nematode signals remains to be determined.

In M. truncatula, the Mtccamk mutant fails to show cytoplasmic aggregation at attempted penetration sites of $C$. trifolii and Phytophthora medicaginis [125], a response that is typically triggered upon fungal contact (including AM fungi) or touch [126]. However, this defect does not affect overall fungal colonization, suggesting that MtCCaMK is not required for infection by these pathogens. In fact, most studies on (hemi)biotrophic pathogenic fungi or oomycetes suggest that such pathogens do not hijack the common symbiosis signaling pathway. In L. japonicus leaf cells, haustorium formation by rust fungi is not dependent on the common symbiosis signaling pathway [127]. Similarly, intracellular colonization of rice roots by the biotrophic rice blast fungus Magnaporthe oryzae does not require OsCCaMK [128]. Also, the oomycete Phytophthora palmivora does not require any components of the common symbiosis signaling pathway to infect and form haustoria in $M$. truncatula roots [129]. Taken together, it appears that pathogenic fungi and oomycetes do not exploit the common symbiosis signaling pathway by producing LCOs, or short chain-chitin oligosaccharides, to facilitate plant colonization. Likewise, no bacterial pathogens have been identified that gained the core nod genes via horizontal gene transfer, allowing them to exploit these to colonize/parasitize potential host plants. This suggests that there may be as 
yet unknown penalties associated with LCO-induced signaling that have been overcome by Glomeromycota and symbiotic rhizobia. It suggests that additional adaptations are required to benefit from LCO signaling. Such adaptations may include the evasion of recognition by the innate immune system. For example, in the case of Glomeromycota, the lack of cell walldegrading enzymes is thought to minimize their perception as potential pathogens [15], and secreted effector proteins have evolved to modulate defense [130]. In case of rhizobium (Sinorhizobium meliloti and Mesorhizobium loti), it was suggested that flagellin, has been adapted so that it does not trigger defense responses [131,132]. Furthermore, rhizobial surface polysaccharides play important roles in the suppression of defense responses in legume roots [133]. Additionally, adaptations in the rhizobial type III secretion system (T3SS) and its associated effector proteins appear to play a key role, which is further discussed below.

\section{From Pathogen to Symbiont}

As lateral gene transfer is a common mechanism in prokaryotes [134], it can be envisioned that new potential symbionts may arise. However, the restricted number of actual rhizobium symbionts suggests that evolution of de novo symbionts is hampered by genetic barriers that affect the relation with potential host plants. To get insights into such barriers, comparative studies of a relatively young symbiont with its nonsymbiotic relatives have been conducted. For this, Cupriavidus taiwanensis is used, as it likely represents a recently evolved nitrogenfixing symbiont. C. taiwanensis is the only symbiotic species in the genus Cupriavidus and sister genus Ralstonia. Cupriavidius and Ralstonia are diverged from Burkholderia and form a distinct lineage in the $\beta$-proteobacteriales $[69,135]$. C. taiwanensis contains a 35-kb symbiotic region on a self-transferrable plasmid. This region contains 31 symbiosis genes organized in operons interspersed with retro-elements, which may be indicative for recent structural rearrangements [136]. C. taiwanensis contains only a single operon of 10 nod genes, nodBCIJHASUQ, that are essential for LCO biosynthesis. This enables $C$. taiwanensis to produce pentameric LCOs with either a vaccenic acid (C18:1) or palmitic acid (C16:0) as lipid tail. Additionally, an O-carbamoyl (determined by nodU), an N-methyl (determined by nodS), and/or 6-O-sulfate group (determined by nodH and nodQ) can be present [136]. This pallet of LCOs is similar to that produced by a broad range of different symbiotic rhizobia in the $\boldsymbol{\alpha}$-proteobacteriales $[137,138]$. But in contrast to several of these species, $C$. taiwanensis has a restricted host range and nodulates only the legume Mimosa pudica. This suggests that the host range of $C$. taiwanensis is constrained by other factors. In search for these factors, it was found that the host-range of $C$. taiwanensis can be extended by mutating its T3SS [139]. Wild-type $C$. taiwanensis forms only infective nodules with degenerative symbiosomes on 
A

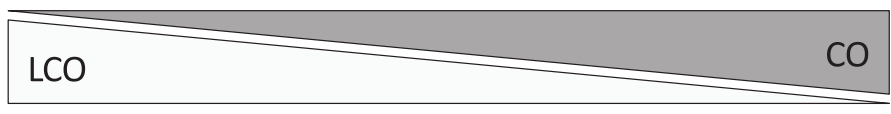

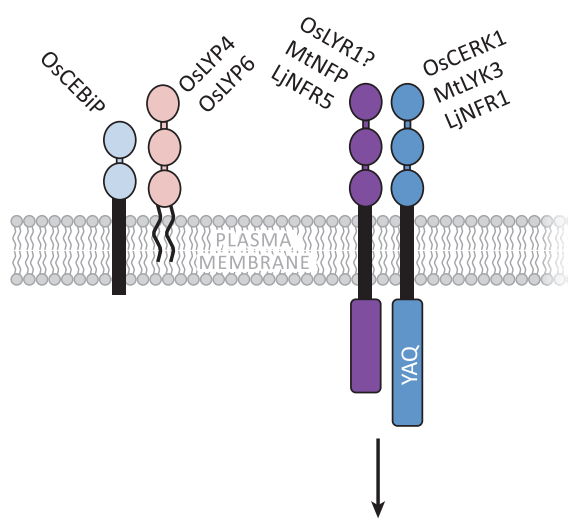

Common symbiosis signaling pathway
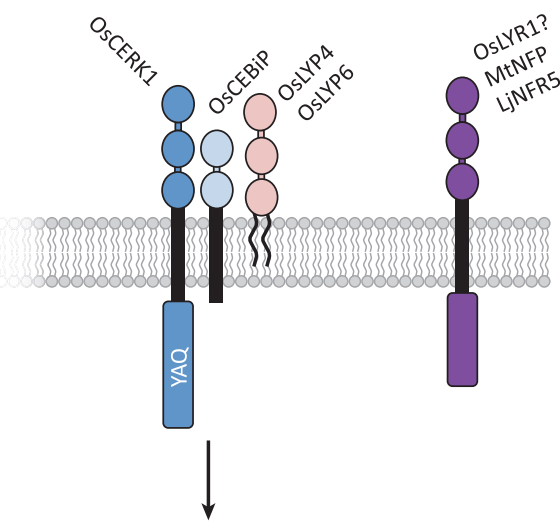

Pathogen-triggered immunity (PTI)

B

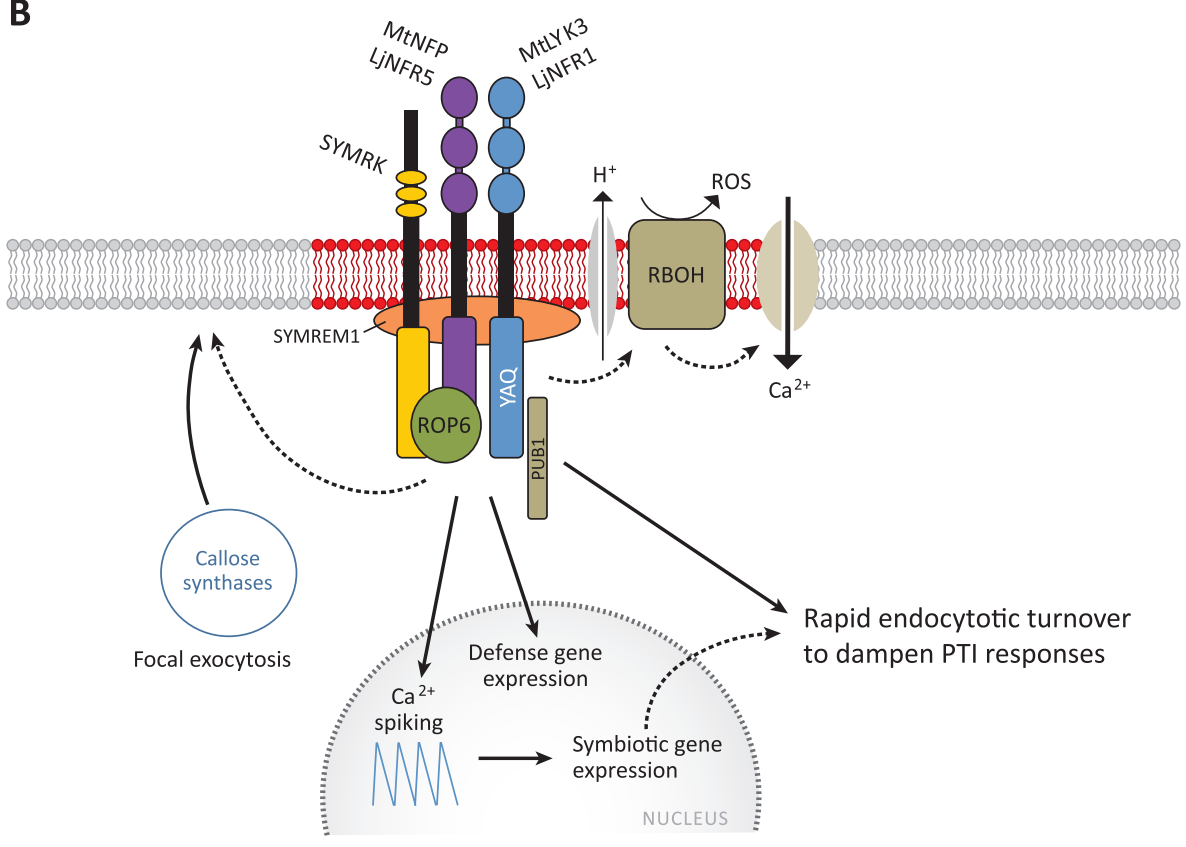


Leucaena leucocephala, whereas the T3SS knockout mutant forms fully functional nodules. This suggests that wild-type $C$. taiwanensis secretes components during its symbiotic lifestyle that hamper symbiosome development in L. leucocephala. Interestingly, the organization of the T3SS operon in C. taiwanensis is atypical for symbiotic rhizobia, but more similar to what is found in the opportunistic pathogen Burkholderia cenocepacia [139]. The biological process in which the T3SS of $C$. taiwanensis functions remains unknown. However, these studies make apparent that the symbiotic lifestyle of $C$. taiwanensis is hampered by a not yet adapted T3SS, thereby restricting its symbiotic potential.

A similar finding was obtained in an experimental evolutionary approach in which the symbiotic plasmid of $C$. taiwanensis was introduced into its relative, Ralstonia solanacearum [140]. $R$. solanacearum is a broad host range plant pathogen that invades roots intercellularly and causes wilting upon colonization of the vascular system [141]. The chimeric $R$. solanacearum produced LCOs but was not able to trigger root nodule formation on M. punica. However, this dramatically changed upon inactivation of its T3SS and the master virulence regulator hrpG. Now the mutated chimeric $R$. solanacearum strain could trigger infected root nodules, although the bacteria did not yet fix nitrogen and triggered

Figure 1. Two hypothetical models to explain the evolutionary conserved dual function of lipochitooligosaccharide (LCO) receptors in symbiosis and immune signaling.

(A) Chitooligosaccharides (COs) and LCOs are perceived by LysM-domain-containing receptors CERK1/ NFR1/LYK3. These LysM-RKs (receptor-like kinases) function as coreceptors in various complexes. Different affinities for the various chitin-like molecules determine which complexes are formed. In rice, chitin perception involves a complex between OsCERK1 and the membrane-anchored CEBiP. Additionally, the GPI-anchored OsLYP4 and OsLYP6 are involved in chitin and peptidoglycan perception. LCOs preferentially recruit the coreceptor CERK1/NFR1/LYK3 into the symbiotic complex at the expense of the complexes involved in MAMP-triggered immune responses. LCO perception involves a complex between CERK1/NFR1/LYK3 and NFR5/NFP-homologs, which have a nonfunctional kinase. This leads to the activation of the common symbiosis signaling pathway, which in turn may affect MAMP-triggered immunity.

(B) Symbiotic LCO receptor complexes activate early immune responses to facilitate symbiont infection. Several early MAMP-triggered immune responses, such as ion changes (including calcium influx), ROS production via respiratory burst oxidases (RBOH), and focal exocytosis, are triggered upon LCO perception and have been implicated in rhizobial infection [188]. LCO receptor heteromer complexes may be formed especially at lipid-raft-like membrane domains (marked in red). Such domains can be marked by the symbiotic remorin SYMREM1 [117] or flotillins [189], which controls their signaling potential and endocytotic turnover to prevent too-strong defense responses. In addition to forming a heteromer complex with the CERK1-orthologous LysM-RK LjNFR1, the kinase-dead LjNFR5 has been found to interact with the processed form of the common symbiosis signaling pathway LRR receptor kinase SYMRK (SYMRK- $\triangle M L D$ ) [82] as well as with the small GTPase ROP6 [81], which control rhizobial infection. Additionally, a U-box E3-ubiquitin ligase (PUB1) has been found to interact with MtLYK3, which may control its ubiquitination-dependent turnover, similar to the role of PUB homologs in immune receptor complexes $[188,173]$. Activation of the common symbiosis signaling pathway, marked by calcium spiking, may further influence the posttranslational turnover of the receptor complexes. 
defense responses in the nodules [140]. Again, this finding illustrates that adaptive changes in the secretome are essential for a successful transfer of the nitrogen-fixing symbiosis trait. Subsequent selection (coculturing) cycles in $M$. pudica further increased the symbiotic capacity of the chimeric $R$. solanacearum, indicating that plants can select mutations in the bacteria that further reduce the activation of host defenses [140,142].

Besides potential negative effects, T3SS-dependent effector proteins can also be used by rhizobium to facilitate symbiosis, and as such play a role as host range determinants in addition to LCOs [143]. The broad host range strain Sinorhizobium fredii NGR234 has been shown to use its T3SS to inject so-called nodulation outer proteins (Nops) into plant cells to suppress defense responses [144,145]. Transcriptional activation of the T3SS-encoding genes was shown to be induced by flavonoids in a NodD-dependent manner, indicating a common regulatory mechanism with LCO biosynthesis genes $[146,147]$. Strikingly, some rhizobia have even evolved T3SS effector proteins that allow them to bypass LCO signaling to enable nodulation. The LCO-deficient Bradyrhizobium elkanii nodC mutant BEnodC was shown to induce nodulation in soybean by making use of its T3SS [148]. This suggests that this strain evolved effectors that when injected into the plant cells can bypass the LCO receptors. It is currently not known how such effectors trigger nodulation. It would be interesting to see whether they signal via the common symbiosis signaling pathway or activate nodulation downstream of this pathway. The LCO-deficient B. elkanii strain failed to form infection threads in roots hairs but instead invaded roots via crack entry [148]. This is in line with a specific role for rhizobium LCO receptors in infection thread formation, as discussed above. Most Bradyrhizobium species rely on LCOs to nodulate, which suggests that this LCO-independent nodulation arose later in evolution. Furthermore, although the LCO-deficient strain could nodulate, its effectiveness was much improved upon addition of LCOs, indicating that LCO-induced signaling is a more efficient strategy.

\section{Perspective: Hormonal Responses May Be Key to Symbiotic Lipochitooligosaccharide Signaling}

The studies described above make clear that symbiotic rhizobia have not only gained the capacity to produce LCOs but also need to reset their T3SS secretome to overcome pathogenicity. Furthermore, we have presented two models that show how perception of LCOs can modulate the balance between different LysM-RK receptor complexes and/ or use the readout of innate immune signaling to benefit rhizobial infection [Figure 1]. It remains unclear, however, to what extent symbiotic LCO signals can also actively repress innate immunity. In this last section, we focus on the potential role of plant hormones in such active repression of innate immunity. On the basis of recent studies, we speculate 
on a central role for DELLA proteins in hormonal cross talk to repress defense responses in symbiosis. However, it should be noted that regulation and cross talk between various hormones, especially in roots, is far from understood.

Symbiotic LCO signaling causes changes in the hormonal landscape of the host plant. This is most obvious in the case of rhizobium-induced nodule organogenesis, but myc-LCOs also induce changes in root architecture. In fact, all classical plant hormones have been implicated in nodulation as well as mycorrhization [149-151], including the major defense hormones salicylic acid (SA), jasmonic acid (JA), and ethylene [152]. Therefore, cross talk between the LCO-induced common symbiosis signaling pathway and various hormonal networks is likely to influence innate immune signaling.

SA is an important regulator of resistance to biotrophic pathogens [153], and SA has also been shown to have a negative effect on both rhizobial and mycorrhizal symbioses [154-158]. AM fungi and rhizobia seem to employ LCO signaling to suppress SA-dependent defense responses. Inoculation of alfalfa (Medicago sativa) plants with LCO-deficient rhizobia or an incompatible rhizobium strain induces accumulation of SA, whereas a slight reduction in SA levels is observed upon inoculation with compatible rhizobia [156]. Likewise, inoculation of tomato with the rather incompatible AM fungal species Glomus mosseae elevates SA concentrations in the root and expression of the SA-responsive gene $P R 1 a$, whereas this is not observed upon inoculation with the very compatible fungal species $R$. irregularis [159]. Studies in pea (Pisum sativum) revealed that mycorrhization induces a transient rise in SA levels, which is repressed during prolonged colonization. In contrast, a constant rise in SA levels is induced in pea plants mutated in CCaMK, suggesting that repression of SA biosynthesis is based on activation of the common symbiosis signalling network [154].

Whereas SA is a major signal in resistance to biotrophic pathogens, defense against necrotrophic pathogens requires JA as signal [152]. Both hormones antagonize each other, such that activation of JA signaling can compromise SA-dependent defense. Several biotrophic pathogens exploit this antagonism to suppress SA-mediated defense [160]. For example, several Pseudomonas syringae strains produce the effector coronatine (COR), which acts as an analog of jasmonate-isoleucine (JA-Ile), the bioactive form of JA [5]. By binding the JA receptor COI1, COR induces proteosomal degradation of the JAZ proteins that function as negative regulators (transcriptional repressors) of JA signaling [152]. CORdeficient strains of $P$. syringae have been shown to produce other effectors to activate $\mathrm{JA}$ responses; e.g. HopX1. HopX1 encodes a cysteine protease that degrades JAZ, thereby activating JA signaling in order to antagonize SA-mediated defense [161]. 
Recently, DELLA proteins have been implicated as important regulators of the JA-SA balance [162]. DELLAs belong to the GRAS family of transcriptional regulators and were originally identified as gibberellin (GA)-sensitive repressors of plant growth $[163,164]$. However, DELLA proteins appear to form signaling hubs involved in various hormone signaling pathways, including in auxin, ethylene, and JA signaling [165]. DELLAs promote JA signaling by competing for JAZ binding with MYC2, a key transcriptional activator of JA signaling $[166,167]$. Activation of JA signaling by MYC2 promotes DELLA accumulation, further attenuating MYC2 repression by JAZ proteins $[168,169]$. Recent data suggest that AM fungi may exploit this DELLA function to reduce SA-mediated defense responses. Experiments in rice and $M$. truncatula revealed that DELLA mutants in rice (Oss/r1) and M. truncatula (Mtdella1/Mtdella2 double mutant) are severely impaired in root infection and/or arbuscule formation $[170,171]$, indicating that DELLA proteins are essential transcriptional regulators of this endosymbiosis. In line with this, GA application at high concentrations was shown to block colonization, whereas lower concentrations were already sufficient to block arbuscule formation [172]. It is therefore tempting to speculate that DELLAs might promote colonization and arbuscule formation through immune modulation by sequestering JAZ proteins, affecting the JA-SA signalling balance.

Ectopic expression in $M$. truncatula of a non-degradable MtDELLA1 allele (Mtdella1- $\Delta 18$ ) in the vasculature and endodermis is sufficient to sustain arbuscule formation in the root cortex of the Mtdella1/Mtdella2 double mutant or upon GA application [170]. Additionally, ectopic expression of Mtdella1- $\Delta 18$ enables arbuscule formation in an L. japonicus Ljcyclops mutant. The CCaMK-interacting protein LjCYCLOPS/MtIDP3 is a key protein of the common symbiotic signaling pathway [42], suggesting that DELLA proteins intersect at some point with LCO signaling but do so in a non-cell-autonomous fashion [170]. Intriguingly, expression of the dominant active Mtdella1- $\Delta 18$ allele in an M. truncatula Mtccamk mutant did not rescue the mycorrhizal colonization defect, which indicates that additional input of CCAMK is required. In rice, the DELLA OSSLR1 was shown to interact with the GRAS family OsDIP1 (DELLA interacting protein 1), which is induced upon mycorrhization [171]. DIP1 was also shown to interact with the GRAS protein RAM1 (required for arbuscular mycorrhization 1), which is essential for root colonization by AM fungi [174]. This led to the suggestion that DELLA, DIP1, and RAM1 are part of a larger GRAS protein regulatory complex [175]. Other GRAS family proteins that play a role in both mycorrhization and nodulation include the transcriptional regulators NSP1 and NSP2 [176-179]. Both proteins are required for the production of strigolactones [178], which act as plant signals that promote hyphal branching of AM fungi as well as root and shoot architecture [180-183]. Both NSP1 and NSP2 have been shown to control mycorrhizal colonization $[26,178,179,184,185]$. However, the relative 
mild effects on mycorrhizal colonization contrasts with the essential role for NSP1 and NSP2 during nodulation $[176,177,186]$. This suggests that different GRAS complexes are involved in the control of the two symbioses. However, our understanding of the functioning of these GRAS complexes in relation to symbiotic LCO signalling remains scant [187] and will remain a major objective in future research.

As has become apparent, the mechanisms behind LCO-mediated modulation of innate immunity are far from clear. However, recent studies not only provided novel insight but also extended the studies to other, nonlegume plant species, such as rice and Arabidopsis. This will open new frontiers to unravel the fundament of symbiotic signaling.

\section{SUMmary POINTS}

1. Combining the nitrogen fixation trait with the capacity to synthesize LCOs marked the origin of nitrogen-fixing rhizobia. The ability to make LCOs enabled rhizobia to activate the signaling pathway that is used by host plants to establish an endosymbiosis with AM fungi. This common symbiosis signaling pathway is essential for the formation of a symbiotic host-microbe interface. In legumes, this signaling pathway evolved to allow formation of root nodules and a more strict control over rhizobium infection.

2. CERK1-type LysM-RKs have dual functions in immune signaling and symbiosis, indicating that LCO signaling and chitin-based immune signaling are intertwined.

3. Two putative models have been put forward to explain this evolutionarily conserved dual function. (I.) Perception of LCOs modulates the balance between different LysMRK receptor complexes, favoring a symbiotic complex at the expense of complexes required for immune responses. (II.) Early immune responses are co-opted to facilitate symbiont infection. Tight regulation of the receptor complexes at the posttranslational level, involving endocytotic turnover, subsequently prevents activation of defense responses.

4. Microbial activation of the common symbiosis signaling pathway is not sufficient to evade innate immunity. Therefore, microbes need to evolve additional genetic adaptations to benefit from LCO signaling. In rhizobia, the T3SS especially needs to be adapted to evade immune responses.

5. Microbial activation of the common symbiosis signaling pathway can modulate innate immune responses through its effect on the hormonal landscape. On the basis of recent studies, we speculate on a central role for DELLA proteins in hormonal cross talk to repress defense responses in symbiosis, in part by influencing the SA-JA balance. 


\section{FUNDING}

R.G. is funded by NWO VICl (865.13.001), R.G. and A.v.Z. by NWO-NSFC Joined Research project (846.11.005), and R.G. and E.L. by European Research Council (ERC-2011AdG294790).

\section{References}

1 Berendsen, R.L. et al. (2012) The rhizosphere microbiome and plant health. Trends Plant Sci. 17, 478-486

2 Mendes, R. et al. (2013) The rhizosphere microbiome: significance of plant beneficial, plant pathogenic, and human pathogenic microorganisms. FEMS Microbiol. Rev. 37, 634-663

3 Gutjahr, C. and Parniske, M. (2013) Cell and developmental biology of arbuscular mycorrhiza symbiosis. Annu. Rev. Cell Dev. Biol. 29, 593-617

4 Zamioudis, C. and Pieterse, C.M.J. (2012) Modulation of host immunity by beneficial microbes. Mol. Plant Microbe Interact. 25, 139-150

5 Jones, J.D.G. and Dangl, J.L. (2006) The plant immune system. Nature 444, 323-329

6 Boller, T. and Felix, G. (2009) A renaissance of elicitors: perception of microbe-associated molecular patterns and danger signals by pattern-recognition receptors. Annu. Rev. Plant Biol. 60, 379-406

7 Pel, M.J.C. and Pieterse, C.M.J. (2013) Microbial recognition and evasion of host immunity. J. Exp. Bot. 64, 1237-1248

8 Streng, A. et al. (2011) Evolutionary origin of rhizobium Nod factor signaling. Plant Signal. Behav. 6, 15101514

9 Svistoonoff, S. et al. (2014) Actinorhizal root nodule symbioses: what is signalling telling on the origins of nodulation? Curr. Opin. Plant Biol. 20, 11-18

10 Redecker, D. et al. (2000) Glomalean fungi from the Ordovician. Science 289, 1920-1921

11 Parniske, M. (2008) Arbuscular mycorrhiza: the mother of plant root endosymbioses. Nat. Rev. Microbiol. 6, 763-775

12 Remy, W. et al. (1994) Four hundred-million-year-old vesicular arbuscular mycorrhizae. Proc. Natl. Acad. Sci. U. S. A. $91,11841-11843$

13 Genre, A. et al. (2005) Arbuscular mycorrhizal fungi elicit a novel intracellular apparatus in Medicago truncatula root epidermal cells before infection. Plant Cell 17, 3489-3499

14 Balestrini, R. and Bonfante, P. (2014) Cell wall remodeling in mycorrhizal symbiosis: a way towards biotrophism. Front. Plant Sci. 5, 237

15 Tisserant, E. et al. (2013) Genome of an arbuscular mycorrhizal fungus provides insight into the oldest plant symbiosis. Proc. Natl. Acad. Sci. U. S. A. 110, 20117-20122

16 Lin, K. et al. (2014) Single nucleus genome sequencing reveals high similarity among nuclei of an endomycorrhizal fungus. PLoS Genet. 10, e1004078

17 Ivanov, S. et al. (2012) Rhizobium - legume symbiosis shares an exocytotic pathway required for arbuscule formation. Proc. Natl. Acad. Sci. U. S. A. 109, 8316-8321

18 Ferrari, S. et al. (2013) Oligogalacturonides: plant damage-associated molecular patterns and regulators of growth and development. Front. Plant Sci. 4, 49

19 Bellincampi, D. et al. (2014) Plant cell wall dynamics and wall-related susceptibility in plant-pathogen interactions. Front. Plant Sci. 5, 228

20 Nadal, M. and Paszkowski, U. (2013) Polyphony in the rhizosphere: presymbiotic communication in arbuscular mycorrhizal symbiosis. Curr. Opin. Plant Biol. 16, 473-479

21 Kosuta, S. et al. (2003) A diffusible factor from arbuscular mycorrhizal fungi induces symbiosis-specific MtENOD11 expression in roots of Medicago truncatula. Plant Physiol. 131, 952-962

22 Kosuta, S. et al. (2008) Differential and chaotic calcium signatures in the symbiosis signaling pathway of legumes. Proc. Natl. Acad. Sci. U. S. A. 105, 9823-9828

23 Kuhn, H. et al. (2010) Membrane steroid-binding protein 1 induced by a diffusible fungal signal is critical for 
mycorrhization in Medicago truncatula. New Phytol. 185, 716-733

Chabaud, M. et al. (2011) Arbuscular mycorrhizal hyphopodia and germinated spore exudates trigger $\mathrm{Ca}^{2+}$ spiking in the legume and nonlegume root epidermis. New Phytol. 189, 347-355

25 Genre, A. et al. (2013) Short-chain chitin oligomers from arbuscular mycorrhizal fungi trigger nuclear $\mathrm{Ca}^{2+}$ spiking in Medicago truncatula roots and their production is enhanced by strigolactone. New Phytol. 198, 179-189

26 Maillet, F. et al. (2011) Fungal lipochitooligosaccharide symbiotic signals in arbuscular mycorrhiza. Nature 469, 58-63

27 Oldroyd, G.E.D. (2013) Speak, friend, and enter: signalling systems that promote beneficial symbiotic associations in plants. Nat. Rev. Microbiol. 11, 252-263

28 Endre, G. et al. (2002) A receptor kinase gene regulating symbiotic nodule development. Nature 417, 962966

29 Stracke, S. et al. (2002) A plant receptor-like kinase required for both bacterial and fungal symbiosis. Nature 417, 959-962

30 Ane, J. et al. (2004) Medicago truncatula DMI1 required for bacterial and fungal symbioses in legumes. Science 303, 1364-1368

31 Imaizumi-Anraku, H. et al. (2005) Plastid proteins crucial for symbiotic fungal and bacterial entry into plant roots. Nature $433,527-531$

32 Riely, B.K. et al. (2007) The symbiotic ion channel homolog DMI1 is localized in the nuclear membrane of Medicago truncatula roots. Plant J. 49, 208-216

33 Peiter, E. et al. (2007) The Medicago truncatula DMI1 protein modulates cytosolic calcium signaling. Plant Physiol. 145, 192-203

34 Saito, K. et al. (2007) NUCLEOPORIN85 is required for calcium spiking, fungal and bacterial symbioses, and seed production in Lotus japonicus. Plant Cell 19, 610-624

35 Kanamori, N. et al. (2006) A nucleoporin is required for induction of $\mathrm{Ca}^{2+}$ spiking in legume nodule development and essential for rhizobial and fungal symbiosis. Proc. Natl. Acad. Sci. U. S. A. 103, 359-364

36 Groth, M. et al. (2010) NENA, a Lotus japonicus homolog of Sec13, is required for rhizodermal infection by arbuscular mycorrhiza fungi and rhizobia but dispensable for cortical endosymbiotic development. Plant Cell 22, 2509-2526

37 Capoen, W. et al. (2011) Nuclear membranes control symbiotic calcium signaling of legumes. Proc. Natl. Acad. Sci. U. S. A. 108, 14348-14353

38 Singh, S. and Parniske, M. (2012) Activation of calcium- and calmodulin-dependent protein kinase (CCaMK), the central regulator of plant root endosymbiosis. Curr. Opin. Plant Biol. 15, 444-453

39 Charpentier, M. et al. (2013) The role of DMI1 in establishing $\mathrm{Ca}^{2+}$ oscillations in legume symbioses. Plant Signal. Behav. 8, e22894

40 Horváth, B. et al. (2011) Medicago truncatula IPD3 is a member of the common symbiotic signaling pathway required for rhizobial and mycorrhizal symbioses. Mol. Plant Microbe Interact. 11, 1345-1358

41 Messinese, E. et al. (2007) A novel nuclear protein interacts with the symbiotic DMI3 calcium- and calmodulin- dependent protein kinase of Medicago truncatula. Mol. Plant Microbe Interact. 20, 912-921

42 Yano, K. et al. (2008) CYCLOPS, a mediator of symbiotic intracellular accommodation. Proc. Natl. Acad. Sci. U. S. A. 105, 20540-20545

43 Delaux, P.-M. et al. (2013) Evolution of the plant-microbe symbiotic "toolkit". Trends Plant Sci. 18, 298-304

44 Wang, B. et al. (2010) Presence of three mycorrhizal genes in the common ancestor of land plants suggests a key role of mycorrhizas in the colonization of land by plants. New Phytol. 186, 514-525

45 Sieberer, B.J. et al. (2012) A switch in $\mathrm{Ca}^{2+}$ spiking signature is concomitant with endosymbiotic microbe entry into cortical root cells of Medicago truncatula. Plant J. 69, 822-830

46 Lerouge, P. et al. (1990) Symbiotic host-specificity of Rhizobium meliloti is determined by a sulphated and acylated glucosamine oligosaccharide signal. Nature 344, 781-784

47 Cooper, J.E. (2007) Early interactions between legumes and rhizobia: disclosing complexity in a molecular dialogue. J. Appl. Microbiol. 103, 1355-1365

48 Xiao, T.T. et al. (2014) Fate map of Medicago truncatula root nodules. Development 141, 3517-3528

49 Popp, C. and Ott, T. (2011) Regulation of signal transduction and bacterial infection during root nodule symbiosis. Curr. Opin. Plant Biol. 14, 458-467 
50 Sprent, J.I. (2007) Evolving ideas of legume evolution and diversity: a taxonomic perspective on the occurrence of nodulation. New Phytol. 174, 11-25

51 Gage, D.J. (2004) Infection and invasion of roots by symbiotic, nitrogen-fixing rhizobia during nodulation of temperate legumes. Microbiol. Mol. Biol. Rev. 68, 280-300

52 Moling, S. et al. (2014) Nod Factor receptors form heteromeric complexes and are essential for intracellular infection in Medicago nodules. Plant Cell 26, 4188-4199

53 Ardourel, M. et al. (1994) Rhizobium meliloti lipooligosaccharide nodulation factors: different structural requirements for bacterial entry into target root hair cells and induction of plant symbiotic developmental responses. Plant Cell 6, 1357-1374

54 Geurts, R. et al. (1997) Sym2 of pea is involved in a nodulation factor-perception mechanism that controls the infection process in the epidermis. Plant Physiol. 115, 351-359

55 Radutoiu, S. et al. (2007) LysM domains mediate lipochitin-oligosaccharide recognition and Nfr genes extend the symbiotic host range. EMBO J. 26, 3923-3935

56 Perret, X. et al. (2000) Molecular basis of symbiotic promiscuity. Microbiol. Mol. Biol. Rev. 64, 180-201

57 Smit, P. et al. (2007) Medicago LYK3, an entry receptor in rhizobial nodulation factor signaling. Plant Physiol. $145,183-191$

58 Sawada, H. et al. (2003) Changing concepts in the systematics of bacterial nitrogen-fixing legume symbionts. J. Gen. Microbiol. 49, 155-179

59 Masson-Boivin, C. et al. (2009) Establishing nitrogen-fixing symbiosis with legumes: how many rhizobium recipes? Trends Microbiol. 17, 458-466

60 Boyd, E.S. and Peters, J.W. (2013) New insights into the evolutionary history of biological nitrogen fixation. Front. Microbiol. 4, 201

61 Raymond, J. et al. (2004) The natural history of nitrogen fixation. Mol. Biol. Evol. 21, 541-554

62 Dos Santos, P.C. et al. (2012) Distribution of nitrogen fixation and nitrogenase-like sequences amongst microbial genomes. BMC Genomics 13, 162

63 Doyle, J.J. (2011) Phylogenetic perspectives on the origins of nodulation. Mol. Plant Microbe Interact. 24, 1289-1295

64 Young, J.P.W. and Haukka, K.E. (1996) Diversity and phylogeny of rhizobia. New Phytol. 133, 87-94

65 Spaink, H.P. et al. (1995) Rhizobium Nodl and NodJ proteins play a role in the efficiency of secretion of lipochitin oligosaccharides. J. Appl. Microbiol. 177, 6276-6281

66 Cardenas, L. et al. (1996) The role of the nodl and nodJ genes in the transport of Nod metabolites in Rhizobium etli. Gene 173, 183-187

67 Fernandez-Lopez, M. et al. (1996) Role of nodl and nodJ in lipo-oligosaccharide secretion in Azorhizobium caulinodans and Escherichia coli. Mol. Microbiol. 20, 993-1000

68 Angeles, L. et al. (2010) Insights into the history of the legume- betaproteobacterial symbiosis. Mol. Ecol. $19,28-30$

69 Bontemps, C. et al. (2010) Burkholderia species are ancient symbionts of legumes. Mol. Ecol. 19, 44-52

70 Aoki, S. et al. (2013) From $\beta$ - to $\alpha$-proteobacteria: the origin and evolution of rhizobial nodulation genes nodIJ. Mol. Biol. Evol. 30, 2494-2508

71 Buist, G. et al. (2008) LysM, a widely distributed protein motif for binding to (peptido)glycans. Mol. Microbiol. 68, 838-847

72 Zhang, X.-C. et al. (2007) Molecular evolution of lysin motif-type receptor-like kinases in plants. Plant Physiol. 144, 623-636

73 Madsen, E.B. et al. (2003) A receptor kinase gene of the LysM type is involved in legume perception of rhizobial signals. Nature 425, 637-640

74 Limpens, E. et al. (2003) LysM domain receptor kinases regulating rhizobial Nod factor-induced infection. Science 302, 630-633

75 Radutoiu, S. et al. (2003) Plant recognition of symbiotic bacteria requires two LysM receptor-like kinases. Nature 425, 585-592

76 Arrighi, J.-F. et al. (2006) The Medicago truncatula lysin motif-receptor-like kinase gene family includes NFP and new nodule-expressed genes. Plant Physiol. 142, 265-279

77 Broghammer, A. et al. (2012) Legume receptors perceive the rhizobial lipochitin oligosaccharide signal molecules by direct binding. Proc. Natl. Acad. Sci. U. S. A. 109, 13859-13864 
78 Madsen, E.B. et al. (2011) Autophosphorylation is essential for the in vivo function of the Lotus japonicus Nod factor receptor 1 and receptor-mediated signalling in cooperation with Nod factor receptor 5. Plant J. $65,404-417$

79 Lefebvre, B. et al. (2012) Role of N-glycosylation sites and CXC motifs in trafficking of Medicago truncatula Nod factor perception protein to plasma membrane. J. Biol. Chem. 287, 10812-10823

80 Pietraszewska-Bogiel, A. et al. (2013) Interaction of Medicago truncatula lysin motif receptor-like kinases, NFP and LYK3, produced in Nicotiana benthamiana induces defence-like responses. PLoS One 8, e65055

$81 \mathrm{Ke}$, D. et al. (2012) The small GTPase ROP6 interacts with NFR5 and is involved in nodule formation in Lotus japonicus. Plant Physiol. 159, 131-143

82 Antolín-Llovera, M. et al. (2014) Cleavage of the SYMBIOSIS RECEPTOR-LIKE KINASE ectodomain promotes complex formation with Nod factor receptor 5. Curr. Biol. 24, 422-427

83 Czaja, L.F. et al. (2012) Transcriptional responses toward diffusible signals from symbiotic microbes reveal MtNFP- and MtDMI3-dependent reprogramming of host gene expression by arbuscular mycorrhizal fungal lipochitooligosaccharides. Plant Physiol. 159, 1671-1685

84 Ben Amor, B. et al. (2003) The NFP locus of Medicago truncatula controls an early step of Nod factor signal transduction upstream of a rapid calcium flux and root hair deformation. Plant J. 34, 495-506

85 Gomez, S.K. et al. (2009) Medicago truncatula and Glomus intraradices gene expression in cortical cells harboring arbuscules in the arbuscular mycorrhizal symbiosis. BMC Plant Biol. 9, 10

86 Gaude, N. et al. (2012) Arbuscule-containing and non-colonized cortical cells of mycorrhizal roots undergo extensive and specific reprogramming during arbuscular mycorrhizal development. Plant J. 69, 510-528

87 Hogekamp, C. et al. (2011) Laser microdissection unravels cell-type-specific transcription in arbuscular mycorrhizal roots, including CAAT-box transcription factor gene expression correlating with fungal contact and spread. Plant Physiol. 157, 2023-2043

88 Op den Camp, R. et al. (2011) LysM-type mycorrhizal receptor recruited for rhizobium symbiosis in nonlegume Parasponia. Science 331, 909-912

89 Behm, J.E. et al. (2014) Parasponia: a novel system for studying mutualism stability. Trends Plant Sci. 19, 757-763

90 Op den Camp, R.H.M. et al. (2012) Nonlegume Parasponia andersonii deploys a broad rhizobium host range strategy resulting in largely variable symbiotic effectiveness. Mol. Plant Microbe Interact. 25, 954-963

91 Fliegmann, J. et al. (2013) Lipo-chitooligosaccharidic symbiotic signals are recognized by LysM receptor-like kinase LYR3 in the legume Medicago truncatula. ACS Chem. Biol. 8, 1900-1906

92 Zhang, X. et al. (2015) The receptor kinase CERK1 has dual functions in symbiosis and immunity signalling. Plant J. 81, 258-267

93 Miyata, K. et al. (2014) Bifunctional plant receptor, OsCERK1, regulates both chitin-triggered immunity and arbuscular mycorrhizal symbiosis in rice. Plant Cell Physiol. 55, 1864-1872

94 Nakagawa, T. et al. (2011) From defense to symbiosis: limited alterations in the kinase domain of LysM receptor-like kinases are crucial for evolution of legume-Rhizobium symbiosis. Plant J. 65, 169-180

95 De Mita, S. et al. (2014) Evolution of a symbiotic receptor through gene duplications in the legumerhizobium mutualism. New Phytol. 201, 961-972

96 Stacey, G. and Shibuya, N. (1997) Chitin recognition in rice and legumes. Plant Soil 194, 161-169

97 Shibuya, N. and Minami, E. (2001) Oligosaccharide signalling for defence responses in plant. Physiol. Mol. Plant Pathol. 59, 223-233

98 Miya, A. et al. (2007) CERK1, a LysM receptor kinase, is essential for chitin elicitor signaling in Arabidopsis. Proc. Natl. Acad. Sci. U. S. A. 104, 19613-19618

99 Wan, J. et al. (2008) A LysM receptor-like kinase plays a critical role in chitin signaling and fungal resistance in Arabidopsis. Plant Cell 20, 471-481

100 Wan, J. et al. (2012) LYK4, a lysin motif receptor-like kinase, is important for chitin signaling and plant innate immunity in Arabidopsis. Plant Physiol. 160, 396-406

101 Petutschnig, E.K. et al. (2010) The lysin motif receptor-like kinase (LysM-RLK) CERK1 is a major chitinbinding protein in Arabidopsis thaliana and subject to chitin-induced phosphorylation. J. Biol. Chem. 285, 28902-28911

102 Shinya, T. et al. (2012) Functional characterization of CEBiP and CERK1 homologs in arabidopsis and rice reveals the presence of different chitin receptor systems in plants. Plant Cell Physiol. 53, 1696-1706 
103 Cao, Y. et al. (2014) The kinase LYK5 is a major chitin receptor in Arabidopsis and forms a chitin-induced complex with related kinase CERK1. eLife 3, e03766

104 lizasa, E. et al. (2010) Direct binding of a plant LysM receptor-like kinase, LysM RLK1/CERK1, to chitin in vitro. J. Biol. Chem. 285, 2996-3004

105 Liu, T. et al. (2012) Chitin-induced dimerization activates a plant immune receptor. Science 336, 1160-1164

106 Kaku, H. et al. (2006) Plant cells recognize chitin fragments for defense signaling through a plasma membrane receptor. Proc. Natl. Acad. Sci. U. S. A. 103, 11086-11091

107 Shimizu, T. et al. (2010) Two LysM receptor molecules, CEBiP and OsCERK1, cooperatively regulate chitin elicitor signaling in rice. Plant J. 64, 204-214

108 Hayafune, M. et al. (2014) Chitin-induced activation of immune signaling by the rice receptor CEBiP relies on a unique sandwich-type dimerization. Proc. Natl. Acad. Sci. U. S. A. 111, E404-E413

109 Narusaka, Y. et al. (2013) Presence of LYM2 dependent but CERK1 independent disease resistance in Arabidopsis. Plant Signal. Behav. 8, 10-12

110 Faulkner, C. et al. (2013) LYM2-dependent chitin perception limits molecular flux via plasmodesmata. Proc. Natl. Acad. Sci. U. S. A. 110, 9166-9170

111 Willmann, R. et al. (2011) Arabidopsis lysin-motif proteins LYM1 LYM3 CERK1 mediate bacterial peptidoglycan sensing and immunity to bacterial infection. Proc. Natl. Acad. Sci. U. S. A. 108, 19824-19829

112 Rey, T. et al. (2013) NFP, a LysM protein controlling Nod factor perception, also intervenes in Medicago truncatula resistance to pathogens. New Phytol. 198, 875-886

113 Ben, C. et al. (2012) Natural diversity in the model legume Medicago truncatula allows identifying distinct genetic mechanisms conferring partial resistance to Verticillium wilt. J. Exp. Bot. 63, 695-709

114 Liang, Y. et al. (2013) Nonlegumes respond to rhizobial Nod factors by suppressing the innate immune response. Science 341, 1384-1387

115 Brewin, N.J. (2004) Plant cell wall remodelling in the rhizobium-legume symbiosis. CRC. Crit. Rev. Plant Sci. 23, 293-316

116 Madsen, L.H. et al. (2010) The molecular network governing nodule organogenesis and infection in the model legume Lotus japonicus. Nat. Commun. 1, 10

117 Lefebvre, B. et al. (2010) A remorin protein interacts with symbiotic receptors and regulates bacterial infection. Proc. Natl. Acad. Sci. U. S. A. 107, 2343-2348

118 Haney, C.H. et al. (2011) Symbiotic rhizobia bacteria trigger a change in localization and dynamics of the Medicago truncatula receptor kinase LYK3. Plant Cell 23, 2774-2787

119 Jarsch, I.K. et al. (2014) Plasma membranes are subcompartmentalized into a plethora of coexisting and diverse microdomains in Arabidopsis and Nicotiana benthamiana. Plant Cell 26, 1698-1711

120 Paparella, C. et al. (2014) The Arabidopsis LYSIN MOTIF-CONTAINING RECEPTOR-LIKE KINASE3 regulates the cross talk between immunity and abscisic acid responses. Plant Physiol. 165, 262-276

121 Liang, Y. et al. (2014) Lipochitooligosaccharide recognition: an ancient story. New Phytol. 204, 289-296

122 Parniske, M. (2000) Intracellular accommodation of microbes by plants: a common developmental program for symbiosis and disease? Curr. Opin. Plant Biol. 3, 320-328

123 Rey, T. and Schornack, S. (2013) Interactions of beneficial and detrimental root-colonizing filamentous microbes with plant hosts. Genome Biol. 14, 121

124 Weerasinghe, R.R. et al. (2005) Root-knot nematodes and bacterial Nod factors elicit common signal transduction events in Lotus japonicus. Proc. Natl. Acad. Sci. U. S. A. 102, 3147-3152

125 Genre, A. et al. (2009) Biotic and abiotic stimulation of root epidermal cells reveals common and specific responses to arbuscular mycorrhizal fungi. Plant Physiol. 149, 1424-1434

126 Hardham, A.R. et al. (2008) Rapid and dynamic subcellular reorganization following mechanical stimulation of Arabidopsis epidermal cells mimics responses to fungal and oomycete attack. BMC Plant Biol. 8, 63

127 Mellersh, D. and Parniske, M. (2006) Common symbiosis genes of Lotus japonicus are not required for intracellular accommodation of the rust fungus Uromyces loti. New Phytol. 170, 641-644

128 Marcel, S. et al. (2010) Tissue-adapted invasion strategies of the rice blast fungus Magnaporthe oryzae. Plant Cell 22, 3177-3187

129 Rey, T. et al. (2015) Medicago truncatula symbiosis mutants affected in the interaction with a biotrophic root pathogen. New Phytol. 206, 497-500

130 Kloppholz, S. et al. (2011) A secreted fungal effector of Glomus intraradices promotes symbiotic biotrophy. 
Curr. Biol. 21, 1204-1209

131 Felix, G. et al. (1999) Plants have a sensitive perception system for the most conserved domain of bacterial flagellin. Plant J. 18, 265-276

132 Lopez-Gomez, M. et al. (2012) Interplay of flg22-induced defence responses and nodulation in Lotus japonicus. J. Exp. Bot. 63, 393-401

133 Fraysse, N. et al. (2003) Surface polysaccharide involvement in establishing the rhizobium-legume symbiosis. Eur. J. Biochem. 270, 1365-1380

134 Darmon, E. and Leach, D.R.F. (2014) Bacterial genome instability. Microbiol. Mol. Biol. Rev. 78, 1-39

135 Vandamme, P. and Coenye, T. (2004) Taxonomy of the genus Cupriavidus: a tale of lost and found. Int. J. Syst. Evol. Microbiol. 54, 2285-2289

136 Amadou, C. et al. (2008) Genome sequence of the $\beta$-rhizobium Cupriavidus taiwanensis and comparative genomics of rhizobia. Genome Res. 18, 1472-1483

137 Lorquin, J. et al. (1997) Sinorhizobium teranga bv. acacia ORS1073 and Rhizobium sp. strain ORS1001, two distantly related Acacia-nodulating strains, produce similar Nod Factors that are O-carbamoylated, $\mathrm{N}$-methylated, and mainly sulfated. J. Bacteriol. 179, 3079-3083

138 Ba, S. et al. (2002) Symbiotic and taxonomic diversity of rhizobia isolated from Acacia tortilis subsp. raddiana in Africa. Syst. Appl. Microbiol. 145, 130-145

139 Saad, M.M. et al. (2012) The type 3 protein secretion system of Cupriavidus taiwanensis strain LMG19424 compromises symbiosis with Leucaena leucocephala. Appl. Environ. Microbiol. 78, 7476-7479

140 Marchetti, M. et al. (2010) Experimental evolution of a plant pathogen into a legume symbiont. PLoS Biol. 8, e1000280

141 Genin, S. and Boucher, C. (2004) Lessons learned from the genome analysis of Ralstonia solanacearum. Annu. Rev. Phytopathol. 42, 107-134

142 Marchetti, M. et al. (2014) Shaping bacterial symbiosis with legumes by experimental evolution. Mol. Plant Microbe Interact. 27, 956-964

143 Marie, C. et al. (2001) Rhizobium type III secretion systems: legume charmers or alarmers? Curr. Opin. Plant Biol. 4, 336-342

144 Bartsev, A. et al. (2004) NopL, an effector protein of Rhizobium sp. NGR234, thwarts activation of plant defense reactions. Mol. Plant Microbe Interact. 134, 871-879

145 Deakin, W.J. and Broughton, W.J. (2009) Symbiotic use of pathogenic strategies: rhizobial protein secretion systems. Nat. Rev. Microbiol. 7, 312-320

146 Wassem, R. et al. (2008) Ttsl regulates symbiotic genes in Rhizobium species NGR234 by binding to tts boxes. Mol. Microbiol. 68, 736-748

147 Krause, A. et al. (2002) Mutational and transcriptional analysis of the type III secretion system of Bradyrhizobium japonicum. Mol. Plant Microbe Interact. 15, 1228-1235

148 Okazaki, S. et al. (2013) Hijacking of leguminous nodulation signaling by the rhizobial type III secretion system. Proc. Natl. Acad. Sci. U. S. A. 110, 17131-17136

149 Ferguson, B.J. and Mathesius, U. (2014) Phytohormone regulation of legume-rhizobia interactions. J. Chem. Ecol. 40, 770-790

150 Foo, E. et al. (2014) Common and divergent roles of plant hormones in nodulation and arbuscular mycorrhizal symbioses. Plant Signal. Behav. 9, e29593

151 Miransari, M. et al. (2014) Plant hormones as signals in arbuscular mycorrhizal symbiosis. Crit. Rev. Biotechnol. 34, 123-133

152 Pieterse, C.M.J. et al. (2012) Hormonal modulation of plant immunity. Annu. Rev. Cell Dev. Biol. 28, 489-521

153 Glazebrook, J. (2005) Contrasting mechanisms of defense against biotrophic and necrotrophic pathogens. Annu. Rev. Phytopathol. 43, 205-227

154 Blilou, l. et al. (1999) Resistance of pea roots to endomycorrhizal fungus or Rhizobium correlates with enhanced levels of endogenous salicylic acid. J. Exp. Bot. 50, 1663-1668

155 Herrera Medina, M. et al. (2003) Root colonization by arbuscular mycorrhizal fungi is affected by the salicylic acid content of the plant. Plant Sci. 164, 993-998

156 Martinez-Abarca, F. et al. (1998) Involvement of salicylic acid in the establishment of the Rhizobium meliloti-alfalfa symbiosis. Mol. Plant Microbe Interact. 11, 153-155

157 Stacey, G. et al. (2006) Effects of endogenous salicylic scid on nodulation in the model legumes Lotus 
japonicus and Medicago truncatula. Plant Physiol. 141, 1473-1481

158 van Spronsen, P.C. et al. (2003) Salicylic acid inhibits indeterminate-type nodulation but not determinatetype nodulation. Mol. Plant Microbe Interact. 16, 83-91

159 López-Ráez, J.A. et al. (2010) Hormonal and transcriptional profiles highlight common and differential host responses to arbuscular mycorrhizal fungi and the regulation of the oxylipin pathway. J. Exp. Bot. 61, 25892601

160 Kazan, K. and Lyons, R. (2014) Intervention of phytohormone pathways by pathogen effectors. Plant Cell 26, 2285-2309

161 Gimenez-Ibanez, S. et al. (2014) The bacterial effector HopX1 targets JAZ transcriptional repressors to activate jasmonate signaling and promote infection in Arabidopsis. PLoS Biol. 12, e1001792

162 Navarro, L. et al. (2008) DELLAs control plant immune responses by modulating the balance of jasmonic acid and salicylic acid signaling. Curr. Biol. 18, 650-655

163 Davière, J.-M. and Achard, P. (2013) Gibberellin signaling in plants. Development 140, 1147-1151

164 Hauvermale, A.L. et al. (2012) Gibberellin signaling: a theme and variations on DELLA repression. Plant Physiol. 160, 83-92

165 Weiss, D. and Ori, N. (2007) Mechanisms of cross talk between gibberellin and other hormones. Plant Physiol. 144, 1240-1246

166 Boter, M. et al. (2004) Conserved MYC transcription factors play a key role in jasmonate signaling both in tomato and Arabidopsis. Genes Dev. 2, 1577-1591

167 Hou, X. et al. (2010) DELLAs modulate jasmonate signaling via competitive binding to JAZs. Dev. Cell 19, 884-894

168 Wild, M. et al. (2012) The Arabidopsis DELLA RGA-LIKE3 is a direct target of MYC2 and modulates jasmonate signaling responses. Plant Cell 24, 3307-3319

169 Yang, D. et al. (2012) Plant hormone jasmonate prioritizes defense over growth by interfering with gibberellin signaling cascade. Proc. Natl. Acad. Sci. U. S. A. 109, E1192-E1200

170 Floss, D.S. et al. (2013) DELLA proteins regulate arbuscule formation in arbuscular mycorrhizal symbiosis. Proc. Natl. Acad. Sci. U. S. A. 110, E5025-E5034

$171 \mathrm{Yu}, \mathrm{N}$. et al. (2014) A DELLA protein complex controls the arbuscular mycorrhizal symbiosis in plants. Cell Res. 24, 130-133

172 El Ghachtouli, N. et al. (1996) First report of the inhibition of arbuscular mycorrhizal infection of Pisum sativum by specific and irreversible inhibition of polyamine biosynthesis or by gibberellic acid treatment. FEBS J. 385, 189-192

173 Mbengue, M. et al. (2010) The Medicago truncatula E3 ubiquitin ligase PUB1 interacts with the LYK3 symbiotic receptor and negatively regulates infection and nodulation. Plant Cell 22, 3474-3488

174 Gobbato, E. et al. (2012) A GRAS-type transcription factor with a specific function in mycorrhizal signaling. Curr. Biol. 22, 2236-2241

175 Gutjahr, C. (2014) Phytohormone signaling in arbuscular mycorhiza development. Curr. Opin. Plant Biol. 20, 26-34

176 Smit, P. et al. (2005) NSP1 of the GRAS protein family is essential for rhizobial Nod factor-induced transcription. Science 308, 1789-1791

177 Kalo, P. et al. (2005) Nodulation signaling in legumes requires NSP2, a member of the GRAS family of transcriptional regulators. Science 308, 1786-1789

178 Liu, W. et al. (2011) Strigolactone biosynthesis in Medicago truncatula and rice requires the symbiotic GRAS-type transcription factors NSP1 and NSP2. Plant Cell 23, 3853-3865

179 Delaux, P. et al. (2013) NSP1 is a component of the Myc signaling pathway. New Phytol. 199, 59-65

180 Akiyama, K. et al. (2005) Plant sesquiterpenes induce hyphal branching in arbuscular mycorrhizal fungi. Nature 435, 824-827

181 Gomez-Roldan, V. et al. (2008) Strigolactone inhibition of shoot branching. Nature 455, 189-194

182 Umehara, M. et al. (2008) Inhibition of shoot branching by new terpenoid plant hormones. Nature 455, 195-200

183 Ruyter-Spira, C. et al. (2011) Physiological effects of the synthetic strigolactone analog GR24 on root system architecture in Arabidopsis: another belowground role for strigolactones? Plant Physiol. 155, 721-734

184 Lauressergues, D. et al. (2012) The microRNA miR171h modulates arbuscular mycorrhizal colonization of 
Medicago truncatula by targeting NSP2. Plant J. 72, 512-522

185 Takeda, N. et al. (2013) CERBERUS and NSP1 of Lotus japonicus are common symbiosis genes that modulate arbuscular mycorrhiza development. Plant Cell Physiol. 54, 1711-1723

186 Heckmann, A.B. et al. (2006) Lotus japonicus nodulation requires two GRAS domain regulators, one of which is functionally conserved in a non-legume. Plant Physiol. 142, 1739-1750

187 Limpens, E. and Bisseling, T. (2014) CYCLOPS: a new vision on rhizobium-induced nodule organogenesis. Cell Host Microbe 15, 127-129

188 Antolín-Llovera, M. et al. (2012) Receptor kinase signaling pathways in plant-microbe interactions. Annu. Rev. Phytopathol. 50, 451-473

189 Haney, C.H. and Long, S.R. (2010) Plant flotillins are required for infection by nitrogen-fixing bacteria. Proc. Natl. Acad. Sci. U. S. A. 107, 478-483 



\section{Chapter 3}

\section{Rhizobium lipochitooligosaccharide signaling triggers accumulation of cytokinins in Medicago truncatula roots}

Arjan van Zeijl, Rik op den Camp, Eva Deinum, Tatsiana Charnikhova, Henk Franssen, Huub op den Camp, Harro Bouwmeester, Wouter Kohlen, Ton Bisseling and Rene Geurts (2015) Rhizobium lipo-chitooligosaccharide signaling triggers accumulation of cytokinins in Medicago truncatula roots. Mol. Plant 8: 1213-1226 


\section{Abstract}

The legume rhizobium symbiosis is initiated upon perception of bacterial secreted lipochitooligosaccharides (LCOS). Perception of these signals by the plant initiates a signalling cascade that leads to nodule formation. Several studies have implicated a function for cytokinin in this process. However, whether cytokinin accumulation and subsequent signalling are an integral part of rhizobium LCO signalling remains elusive. Here, we show that cytokinin signalling is required for the majority of transcriptional changes induced by rhizobium LCOs. In addition, we demonstrate that several cytokinins accumulate in the root susceptible zone three hours post rhizobium LCO application, including the biologically most active cytokinins trans-zeatin and isopentenyl adenine. These responses are dependent on calcium- and calmodulin-dependent protein kinase (CCaMK), a key protein in rhizobial LCO induced signalling. Analysis of the ethylene insensitive Mtein2/Mtsickle mutant showed that LCO-induced cytokinin accumulation is negatively regulated by ethylene. Together with transcriptional induction of ethylene biosynthesis genes, it suggests a feedback loop negatively regulating LCO signalling and subsequent cytokinin accumulation. We argue that cytokinin accumulation is a key step in the pathway leading to nodule organogenesis and that this is tightly controlled by feedback loops. 


\section{INTRODUCTION}

Legumes can engage endosymbiotically with different nitrogen-fixing rhizobial species. Upon signal exchange between host and microsymbiont, a developmental program is initiated that gives rise to a new organ, the root nodule [1-4]. These nodules are colonized intracellularly, providing a niche to the bacteria optimal for the fixation of atmospheric nitrogen. The key signal molecules that initiate nodule organogenesis are bacterial secreted lipochitooligosaccharides (LCOs), also known as Nodulation (Nod) factors [5]. In some legumes, these bacterial signals are even sufficient to trigger the complete developmental program resulting in formation of (empty) nodules [6-9]. Rhizobium LCOs are perceived by LysM domain receptor kinases at the root epidermis [10-13]. This activates a signalling cascade consisting of a plasma membrane localized LRR-type receptor kinase (named MtDMI2 in Medicago truncatula), a cation ion channel (named MtDMI1 in M. truncatula) and several components of the nuclear pore complex [14]. Activation of this signalling cascade results in nuclear calcium oscillations, which are interpreted by a nuclear localized calciumand calmodulin-dependent protein kinase (CCaMK, named MtDMI3 in M. truncatula) [15, 16]. CCaMK activates the transcriptional regulator CYCLOPS, which subsequently activates transcription of downstream targets, including the transcription factor NODULE INCEPTION (NIN) [17].The rhizobium LCO signalling pathway interacts with plant hormone homeostasis at multiple levels. For example, activation of cytokinin phosphorelay signalling is essential for nodule formation, whereas ethylene signalling is known to inhibit this process [18-20]. Unravelling how these hormonal pathways are integrated in symbiotic LCO signalling is key to understand how legume plants establish symbiosis with rhizobium.

Ethylene negatively regulates rhizobium LCO-induced signalling and subsequent nodule formation. The first and rate-limiting step in ethylene biosynthesis is conversion of $S$-adenosyl-L-methionine (S-AdoMet) into 1-aminocyclopropane-1-carboxylic acid (ACC) by ACC synthases (ACS) [21]. Subsequently, ACC is converted into ethylene by ACC oxidase (ACO) activity [21]. Interestingly, in pea (Pisum sativum) roots, ACO genes are expressed in pericycle, endodermal and inner cortical cells opposite phloem poles, limiting nodule initiation to opposite protoxylem poles [22]. Ethylene is perceived by a set of ER-localized receptors that control activity of the ER-localized EIN2 protein. EIN2 forms a central component of the ethylene signalling pathway [21, 23, 24]. Upon activation, EIN2 is cleaved, by which its C-terminal domain is released and can move to the nucleus to modulate transcription [25]. The Mtein2 mutant in M. truncatula, named Mtsickle, is ethylene insensitive and displays hyper nodulation upon rhizobium inoculation, forming up to ten times more nodules in a distinct zone $[26,27]$. Ethylene has been shown to affect LCO signalling at an early step in 
the signalling cascade, most likely upstream or at the point of calcium oscillation. This is based on the observation that, in presence of ethylene, an increased LCO concentration is required to initiate this cellular calcium response. Furthermore, a decrease in oscillation frequency is observed in Mtein2/Mtsickle compared to wild type [19].

In contrast to ethylene, cytokinin acts as an important positive regulator of nodule organogenesis. This was first illustrated by physiological studies, which showed that external application of cytokinin triggers the formation of nodule-like structures on the roots of several legume plants [28-31]. Genetic studies revealed involvement of a specific cytokinin receptor in nodulation: MtCRE1 and LjLHK1 in M. truncatula and Lotus japonicas, respectively. Both receptors are putatively orthologous to the arabidopsis (Arabidopsis thaliana) AtAHK4/ AtCRE1 cytokinin receptor, which functions redundantly to cytokinin receptors AtAHK2 and AtAHK3 [32-37]. Not only are MtCRE1 and LjLHK1 essential for nodule organogenesis; gain-of-function mutations that make these receptors hypersensitive to cytokinin lead to spontaneous nodule formation [20,38]. Several symbiotic genes have been reported to be responsive to cytokinin signalling, including the transcriptional regulators NSP1, NSP2, ERN1 and NIN [37]. Rhizobium LCO signalling also induces expression of typical cytokinin responsive genes such as type-A Response Regulators [35-37, 39]. This further underlines integration of cytokinin phosphorelay signalling in the LCO signalling pathway.

Naturally occurring cytokinins are $N^{6}$-substituted adenine derivatives that can be classified as either aromatic or isoprenoid, depending on their side chain. Of these, the isoprenoidtype cytokinins are most abundant in plants and their biosynthesis is well studied $[40,41]$. The rate-limiting step in the biosynthesis of isoprenoid-type cytokinins is catalysed by the isopentenyl transferases (IPTs), which can be classified into two groups depending on their substrate. Adenylate-IPTs use adenosine 5'-phosphate (AMP, ADP or ATP) as substrate and are involved in the production of isopentenyl adenine (iP), trans-zeatin ( $\mathrm{tZ}$ ) and dihydrozeatin (DZ), whereas tRNA-IPTs use tRNAs as substrate and are involved in biosynthesis of ciszeatin (cZ). Biosynthesis of tZ-type cytokinins involves hydroxylation of iP-nucleotides by a group of cytochrome P450 monooxygenases, which in arabidopsis are named AtCYP735A1 and AtCYP735A2 [42]. Release of bioactive cytokinins from their nucleotide precursors is catalysed by a group of $5^{\prime}$-monophosphate phosphoribohydrolases, referred to as LONELY GUYS (LOGs) $[43,44]$. Cytokinin oxidases/dehydrogenases (CKXs) are involved in cytokinin degradation [45], whereas adenine phosphoribosyl transferases (APTs) inactivate cytokinin by converting them back to their nucleotide form [46]. Recently, several studies reported a link between cytokinin biosynthesis genes and nodule formation $[47,48]$. In $L$. japonicus, LJIPT3 expression increases within three hours after rhizobial inoculation, and 
in M. truncatula both MtLOG1 and MtLOG2 are up-regulated during later stages of nodule formation. Moreover, RNA interference-mediated knock-down of LjIPT3 and MtLOG1 leads to a reduction in the total number of nodules formed per plant $[47,48]$. However, what remains unknown is whether cytokinins are produced as a signalling intermediate upon rhizobium LCO perception and to what extent this contributes to rhizobium LCO-induced signalling.

In this study, we used RNA sequencing to determine the role of cytokinin in rhizobium LCO-induced signalling in M. truncatula. This revealed that the majority of transcriptional changes induced three hours post rhizobium LCO application are dependent on the cytokinin receptor, MtCRE1. Furthermore, we show that LCO treatment induces expression of cytokinin as well as ethylene biosynthesis genes. Measurements of the cytokinin concentration in $M$. truncatula roots showed an accumulation of bioactive cytokinins three hours after rhizobium LCO application. The latter response is inhibited by ethylene, suggesting the presence of a negative feedback loop.

\section{RESULTS}

The Majority of Rhizobium LCO-Induced Transcriptional Changes Are MtCRE1-Dependent Activation of the cytokinin receptor MtCRE1/LjLHK1 is both sufficient and essential to trigger nodule organogenesis $[18,20]$. However, the temporal and mechanistic regulation of the cytokinin signalling pathway upon rhizobium LCO signalling remains unknown. To gain insight in the regulation of this signalling pathway, we studied the role of MtCRE1 in rhizobium LCO-induced gene expression by RNA sequencing (RNA-seq). Three biological replicates of $M$. truncatula wild type and Mtcre1 mutant were grown vertically on agar-solidified Fåhraeus medium $\left(0.2 \mathrm{mM} \mathrm{Ca}\left(\mathrm{NO}_{3}\right)_{2}\right)$ supplemented with $1 \mu \mathrm{M}$ amino ethoxyvinylglycine (AVG), commonly used in in vitro nodulation assays to increase nodulation ability [e.g. 49, 50-53]. Seedling roots were treated with Sinorhizobium meliloti LCOs $\left(\sim 10^{-9} \mathrm{M}\right)$ for three hours, and RNA was isolated from the zone of the root susceptible to rhizobium infection. This zone, hereafter referred to as the susceptible zone, encompasses a region of $\sim 5 \mathrm{~mm}$ and is analogous to the distal region of the elongation zone and the entire differentiation zone of the root. We chose to sample after three hours, as this time point represents a stage in the interaction at which cytokinin-dependent transcriptional changes are reported but mitotic activation of pericycle and cortical cells is not yet occurring [36, 37, 54]. As cytokinin signalling is important for root development [55], we first compared the transcriptomes of mock treated wild-type and Mtcre1 mutant plants. This revealed 69 genes that display a significant transcriptional difference using a minimal fold change (FC) larger than two and 


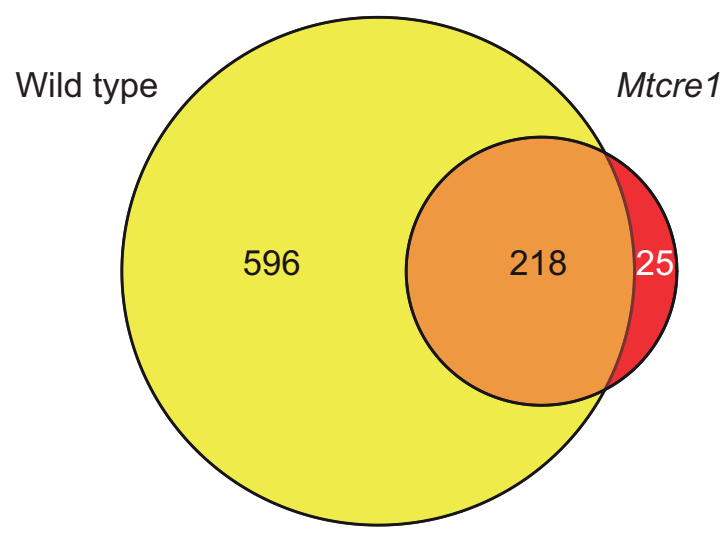

Figure 1. The majority of transcriptional changes induced three hours after rhizobium LCO treatment depend on MtCRE1.

A Venn diagram showing the number of genes differentially expressed (FC $>2, p<0.001$ ) in the root susceptible zone of wild-type $M$. truncatula and Mtcre 1 mutant upon rhizobium LCO treatment $\left(10^{-9}\right.$ $\mathrm{M}, 3 \mathrm{~h}$ ). Gene expression was determined by RNA-seq of three biological replicates per treatment/ genotype combination. Yellow, orange and red indicate the number of genes differentially expressed in wild type only, both wild type and Mtcre1 and Mtcre1 only, respectively.

a Bonferroni-corrected $p$-value smaller than 0.001 (Supplemental Table 1). This limited number of differentially expressed genes between wild type and Mtcre1 is consistent with the absence of any obvious root phenotype in Mtcre1 [37]. Using the same criteria, we identified 814 genes in $M$. truncatula wild-type roots that display differential expression upon rhizobium LCO signalling. Of these genes, 609 were up-regulated and 205 were downregulated (Supplemental Table 2). Among the up-regulated genes are MtENOD11 and MtERN1 as well as the cytokinin responsive genes MtNIN, MtCLE13, MtRR4, MtRR5, MtRR8, MtRR9 and MtRR11 that were found in previous studies (Supplemental Figure 1) [35, 36, 56-58]. In case of the Mtcre1 mutant, only 243 genes were differentially expressed upon LCO application (233 up-regulated and 10 down-regulated). Comparing the transcriptional changes observed in wild type and Mtcre1 mutant after rhizobium LCO application demonstrates that a large set of 596 genes, which corresponds to $\sim 73 \%$ of the genes differentially expressed in wild type, are dependent on MtCRE1-mediated signalling (FC > $2, p<0.001$ ) (Figure 1). Twenty-five genes showed significant differential expression upon LCO treatment in the Mtcre1 mutant specifically (FC $>2, p<0.001)$ (Supplemental Table 3).

To validate our data, the expression of the set of differentially regulated genes is compared to that on the Medicago Gene Expression Atlas [59]. To this end, an averaged expression was calculated over several studies comparable to ours, consisting of samples taken at six and 24 
hours after LCO treatment and one, three and five days after rhizobium inoculation [59-61]. Of the 814 differentially regulated genes identified in wild-type $M$. truncatula, 623 genes are represented by at least one unique probe on the array. Of these, $82 \%$ (508 genes) behave similar in the reported microarray studies (Supplemental Figures 2-3). Approximately $10 \%$ (63 genes) of the genes responded exclusively in our experiment, whereas $\sim 8 \%$ (52 genes) responds differently between both sets (Supplemental Figure 2 and Supplemental Table 5). Overall, this comparison suggests that the RNA-seq data are in line with previous studies.

Gene Ontology (GO) enrichment analysis identified a significant overrepresentation of $48 \mathrm{GO}$ terms associated with genes differentially regulated in wild-type $M$. truncatula specifically $(p<0.05)$. These included phosphorelay response regulator activity, adenine phosphoribosyl transferase activity and 1-aminocyclopropane-1-carboxylate synthase activity (Supplemental Table 6). This points to an induction of cytokinin signalling, cytokinin turnover and ethylene biosynthesis, respectively. The same analysis on genes differentially expressed in both wild type and Mtcre1 mutant after rhizobium LCO exposure revealed 27 significantly enriched GO terms $(p<0.05)$ (Supplemental Table 7). Among these are GO terms associated with chitin breakdown and phosphorylation of inositol phosphates. These data show that rhizobium LCO signalling induces rapid transcriptional reprogramming, which to a large extent is dependent on a functional MtCRE1 cytokinin receptor. This implies that cytokinin has a key function at the preinfection stage of the rhizobium-legume interaction, three hours after rhizobium LCO perception.

\section{Spatiotemporal Localization of the Cytokinin Response}

Since cytokinin signalling is an essential step in nodule formation, we questioned in which cell layers cytokinin signalling is induced upon rhizobium LCO perception. To this end, we used the synthetic cytokinin reporter TCS driving $\beta$-glucuronidase (TCS:GUS) [62]. This chimeric cytokinin reporter construct was introduced in M. truncatula roots using Agrobacterium rhizogenes-mediated transformation. Spatiotemporal expression analysis revealed a moderate level of variation between individual transgenic roots carrying TCS:GUS. Despite this level of variation, faint expression of the TCS:GUS reporter is observed in some, but not all, pericycle cells in mock-treated roots. This is consistent with observations in arabidopsis, where expression of the TCS reporter was observed in xylem pole pericycle cells specifically [63]. Application of rhizobium LCOs $\left(\sim 10^{-9} \mathrm{M}\right)$ increased expression of the TCS:GUS reporter in the susceptible zone specifically in $\sim 30 \%$ of the transgenic roots $(n=12 / 38)$. Sectioning of these root segments revealed induction of TCS:GUS in the cortex, endodermis and pericycle

(Figure 2). This suggests that cytokinin acts as a non-cell-autonomous signal, during the early stages of the legume-rhizobium interaction. 


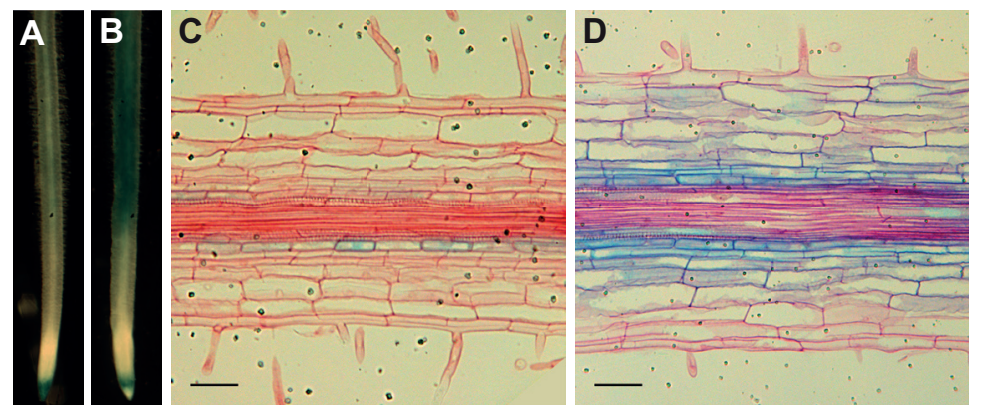

Figure 2. Expression of the synthetic cytokinin responsive reporter TCS:GUS in M. truncatula transgenic roots upon application of rhizobium LCOs.

(A-B) Whole mount images of mock-treated (A) or rhizobium LCOs-treated $\left(10^{-9} \mathrm{M}, 3 \mathrm{~h}\right)(\mathrm{B})$ transgenic roots stained for GUS activity. Transgenic roots were generated by Agrobacterium rhizogenes-mediated transformation.

(C-D) Sections through the susceptible zone of roots shown in (A) and (B), respectively. Sections are counter stained with ruthenium red. Scale bars represent $50 \mu \mathrm{m}$.

\section{Rhizobium LCO Signalling Induces a Change in the Bioactive Cytokinin Pool}

Mining the transcriptome data, we noted differential expression of genes involved in cytokinin biosynthesis and/or activation. A putative IPT (Medtr2g022140), CYP735A (Medtr6g017325) and LOG (Medtr1g057020) homolog were induced three hours after LCO application (Figure 3A). We refer to these genes as MtIPT1, MtCYP735A1 and MtLOG3 based on consecutive numbering in relation to a previous study [48]. Expression of MtCYP735A1 is induced $\sim 6$-fold in wild type and $\sim 2.5$-fold in Mtcre1 after LCO application (Figure 3A), although induction in Mtcre1 was not found to be significant when using the stringent correction for multiple testing. This suggests that LCO-induced expression of MtCYP735A1 may be partly dependent on MtCRE1. By contrast, MtIPT1 expression is induced 4 -fold in both wild-type $M$. truncatula and Mtcre1 mutant, indicating that induction of this gene by rhizobium LCOs is MtCRE1 independent. This suggests that rhizobium LCO-induced signalling leads to (local) cytokinin biosynthesis. Besides cytokinin synthesis genes, genes involved in cytokinin metabolism are also induced by rhizobium LCOs (Figure 3B). These include two putative APT (Medtr3g106780, Medtr5g012210) and two putative CKX (Medtr4g126150, Medtr2g039410) homologs, referred to as MtAPT1, MtAPT2 and MtCKX2, MtCKX3, respectively [58]. These genes are induced in wild-type $M$. truncatula but not Mtcre1. As MtLOG3 is also induced in an MtCRE1-dependent manner, this suggests a set of feedback loops tightly regulating LCO-induced cytokinin levels. Therefore, these data indicate that LCO signalling may induce a rapid change in the cytokinin concentration in the $M$. truncatula root susceptible zone. 

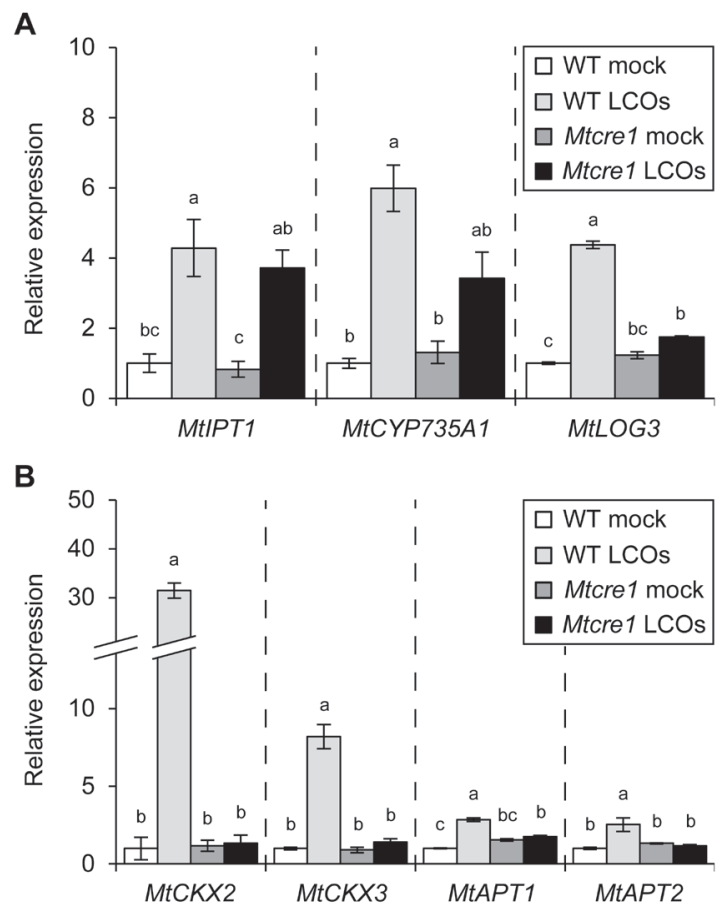

C

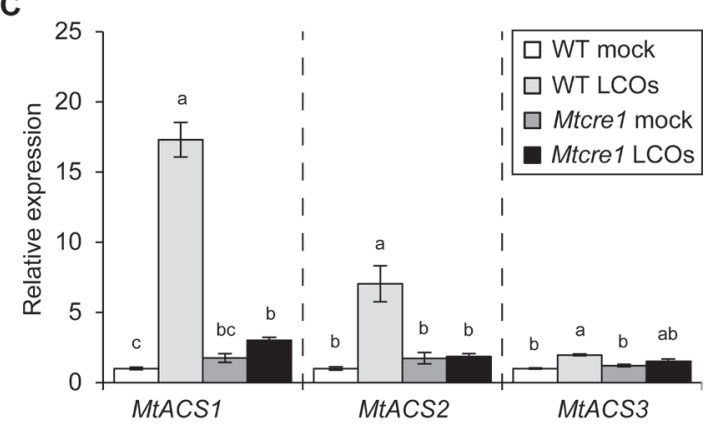

Figure 3. Rhizobium LCO signalling induces expression of genes involved in cytokinin and ethylene biosynthesis.

(A) Expression of genes involved in cytokinin biosynthesis in wild-type M. truncatula and Mtcre1 mutant.

(B) Expression of genes involved in cytokinin turnover in wild-type M. truncatula and Mtcre1 mutant.

(C) Expression of ACC synthases (ACS) in wild-type M. truncatula and Mtcre1 mutant.

Expression was determined at three hours after rhizobium LCO treatment $\left(10^{-9} \mathrm{M}\right)$ (or mock) using RNA-seq and normalized to that in the mock-treated wild type. Expression is shown for genes that were found to be differentially expressed (FC $>2, p<0.001)$ in wild type after LCO treatment. Data shown represent the means of three biological replicates \pm SEM. Different letters above the bars indicate a significant difference $(p<0.001)$. 
A

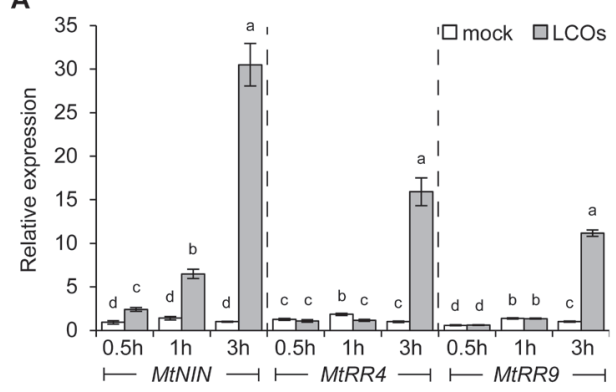

B

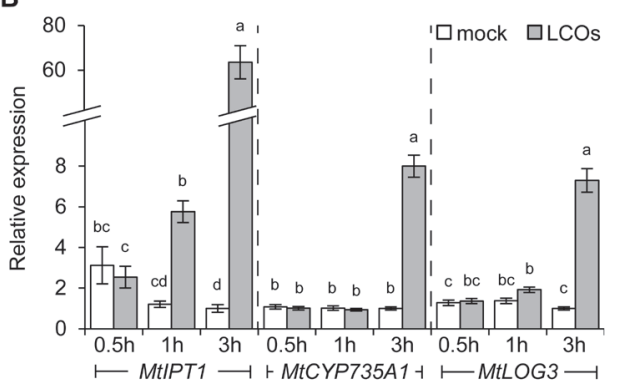

C

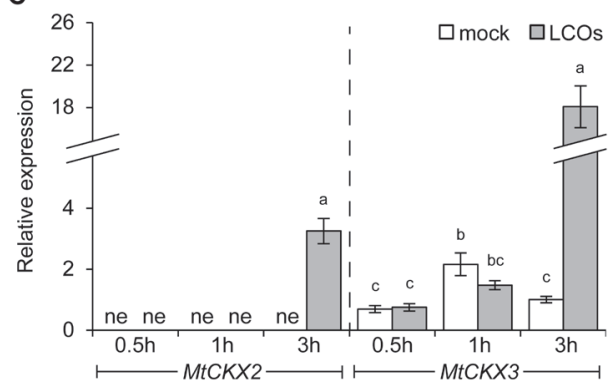

D

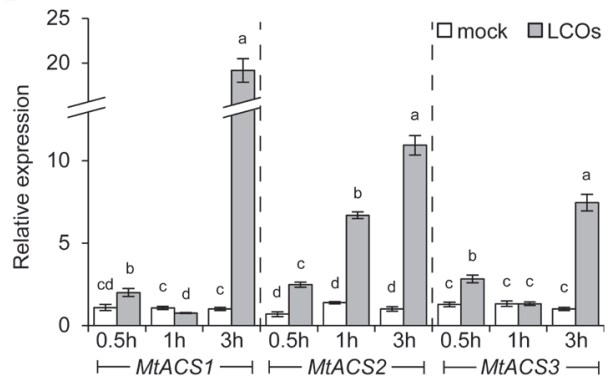

Figure 4. Time course experiment of LCO-responsive genes.

(A) Expression of MtNIN and cytokinin response regulators MtRR4 and MtRR9.

(B) Expression of cytokinin biosynthesis genes MtIPT1, MtCYP735A1 and MtLOG3.

(C) Expression of cytokinin oxidase/dehydrogenases MtCKX2 and MtCKX3.

(D) Expression of ethylene biosynthesis genes MtACS1-3.

Expression was determined at 30 minutes $(0.5 \mathrm{~h})$, one hour $(1 \mathrm{~h})$ and three hours $(3 \mathrm{~h})$ after rhizobium LCO treatment $\left(10^{-9} \mathrm{M}\right)$ (or mock) using qRT-PCR and normalized to the 3-h mock sample. ne indicates genes not expressed under the conditions specified. Data shown represent the means of three biological replicates \pm SEM. Different letters above the bars indicate a significant difference $(p<0.01)$.

To obtain better insight in the regulation of cytokinin biosynthesis genes by rhizobium LCOs, we studied their expression at different time points (30 minutes, one hour and three hours after LCO exposure), and in absence or presence of the ethylene synthesis inhibitor AVG, using quantitative RT-PCR (qRT-PCR). LCO responsiveness of these samples was confirmed by strong induction of MtNIN (Figures 4A and 5A). Analysis of MtIPT1 expression showed this gene is first induced at one hour after LCO treatment, whereas expression of MtCYP735A1 and MtLOG3 is only induced at three hours after LCO exposure (Figure 4B). Expression of MtIPT1 and MtCYP735A1 is induced in both the presence and absence of AVG (Figure 5B), which excludes that induction of both cytokinin biosynthesis genes by rhizobium LCOs results from low ethylene levels. Analysis of the expression of the cytokinin oxidases MtCKX2 and MtCKX3 and the cytokinin response regulators MtRR4 and MtRR9 shows that cytokinin responses are first induced at three hours after LCO exposure (Figure 
4A, C). Furthermore, our data indicate that induction of cytokinin signalling by rhizobium LCOs might be inhibited by ethylene, because in the absence of AVG only MtRRg but not MtRR4 expression is induced, and this response is less in the absence of AVG ( 3.5- vs 10fold, respectively) (Figure 5A). These data suggest rapid activation of cytokinin biosynthesis upon rhizobium LCO perception.

To test whether rhizobium LCO perception induces a change in the cytokinin concentration, we measured cytokinin levels in the $M$. truncatula root susceptible zone of wild type and the Mtccamk/Mtdmi3 mutant at three hours after LCO exposure using ultra-performance liquid chromatography coupled to tandem mass spectrometry (UPLC-MS/MS). For this, we focused on isoprenoid-type cytokinins because these are the predominant form found in plants [40]. Of all cytokinins measured, isopentenyl adenine (iP), iP-riboside (iPR), transzeatin (tZ), cis-zeatin (cZ), tZ-9-glycosylated (tZ9G) and cZ-riboside (cZR) could be detected and quantified. No change in the levels of $c Z, t Z 9 G$ and $c Z R$ were detected after LCO exposure (Supplemental Figure 4). By contrast, the amounts of iP, iPR and tZ did increase significantly $\sim 15,2.5$ and 8 times, respectively, in wild type upon LCO application ( $p<0.05$, Figure $6 \mathrm{~A}-\mathrm{C}$ ). This response is dependent on a functional LCO signaling cascade as it was not observed in the Mtccamk/Mtdmi3 mutant (Figure 6A-C). These results demonstrate that a number of cytokinins accumulate within three hours after rhizobium LCO perception, including the biologically most active cytokinins iP and tz [41].

To position the cytokinin accumulation in the rhizobium LCO signalling pathway, we analysed this response in the Mtnsp1, Mtnsp2 and Mtnsp1Mtnsp2 double mutant. In contrast to MtNSP2, expression of MtNSP1 in the M. truncatula wild-type root is induced $~ 5$-fold after exposure to rhizobium LCOs, whereas only a 2.5 -fold induction is observed in the Mtcre 1 mutant (Supplemental Figure 5). This indicates that LCO-induced MtNSP1 expression is partly dependent on cytokinin signalling. Next, we measured the cytokinin levels in the Mtnsp1 and Mtnsp 2 mutants and compared them with wild type. Again we observed accumulation of iP, tZ and iPR in wild-type M. truncatula (Figure 6). Measurements in the Mtnsp1, Mtnsp2 and Mtnsp1Mtnsp2 mutant roots showed that basal cytokinin levels were similar to those in wild type (Figure 6D-F). As in wild type, LCO treatment induced an accumulation of cytokinin in Mtnsp1, Mtnsp2 and Mtnps1Mtnsp2. However, in these mutants, accumulation of iP and tZ is approximately $60-75 \%$ and $30-40 \%$ of that in the wild type, respectively (Figure 6D-F). Taken together, we demonstrated that rhizobium LCO signalling triggers rapid accumulation of bioactive cytokinin in a CCaMK-dependent manner. This response is controlled only partially by the GRAS-type regulators NSP1 and/or NSP2, possibly in a positive feedback loop. 

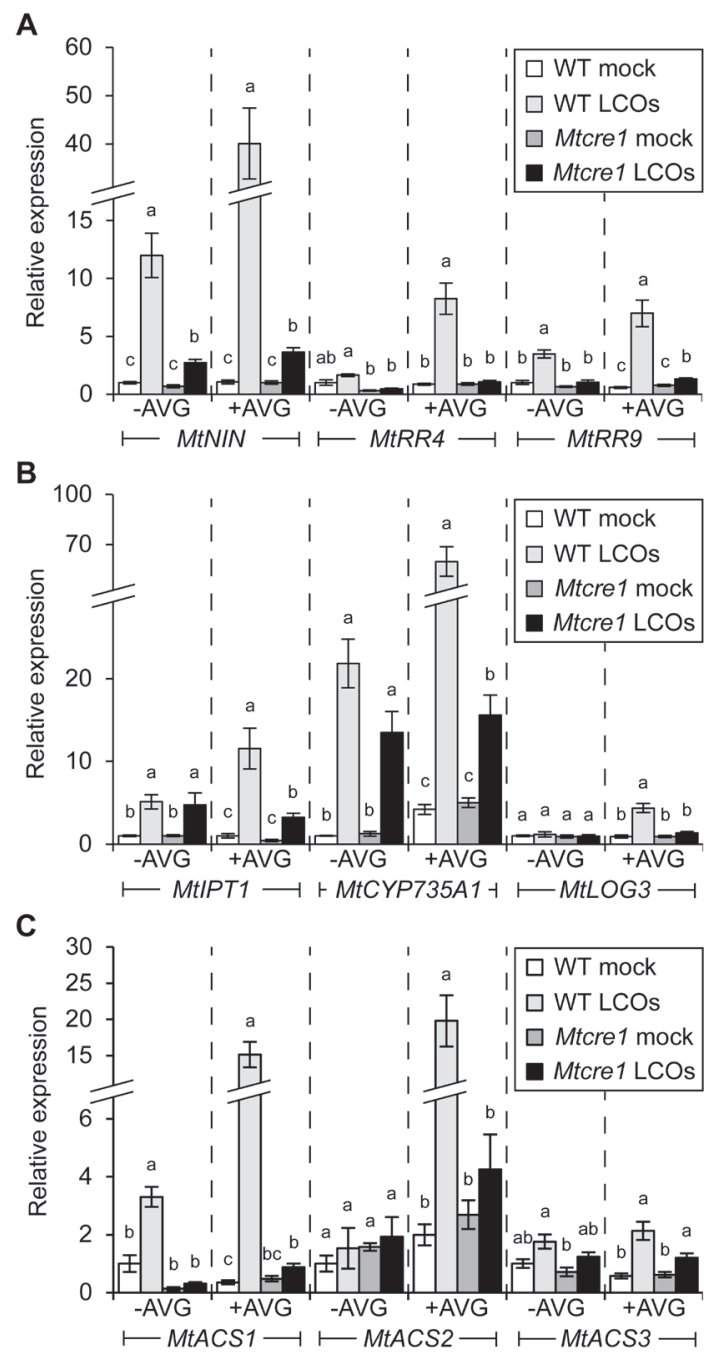

Figure 5. Expression of LCO-responsive genes in the absence or presence of the ethylene synthesis inhibitor AVG.

(A) Expression of MtNIN and cytokinin response regulators MtRR4 and MtRR9.

(B) Expression of cytokinin biosynthesis genes MtIPT1, MtCYP735A1 and MtLOG3.

(C) Expression of ethylene biosynthesis genes MtACS1-3.

M. truncatula wild-type and Mtcre1 mutant plants were grown on agar-solidified Fåhraeus medium with or without $1 \mu \mathrm{M}$ AVG. Expression was determined at three hours after rhizobium LCO treatment $\left(10^{-9} \mathrm{M}\right)$ (or mock) using qRT-PCR and normalized to that in the mock-treated wild type grown in the absence of AVG. Data shown represent the means of three biological replicates \pm SEM. Different letters above the bars indicate a significant difference $(p<0.01)$. 

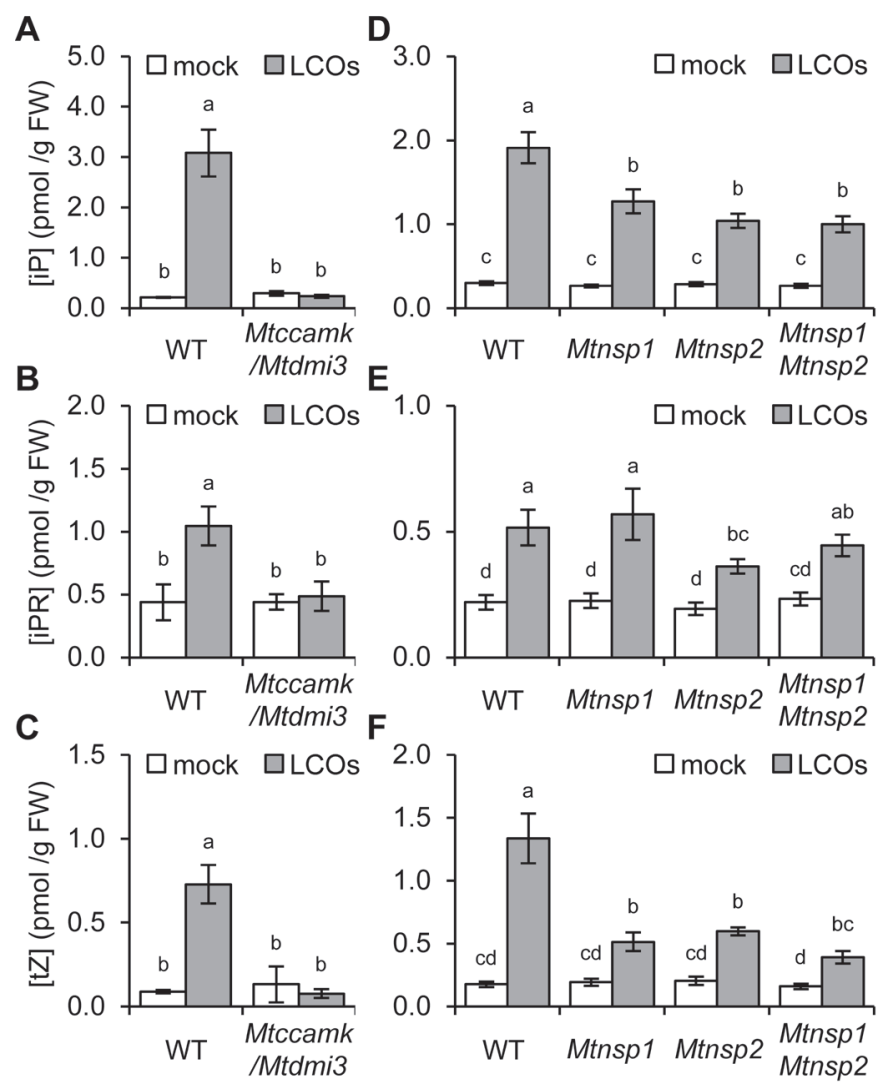

Figure 6. Cytokinins accumulate in the M. truncatula root susceptible zone upon S. meliloti LCO signalling.

(A-C) Concentrations of the cytokinins isopentenyl adenine (iP) (A), isopentenyl adenine riboside (iPR) (B) and trans-zeatin (tZ) (C) in root susceptible zones of the M. truncatula wild type and Mtccamk/ Mtdmi3 mutant treated with mock or rhizobium LCOs $\left(10^{-9} \mathrm{M}, 3 \mathrm{~h}\right)$. Data represent the means of four biological replicates (two for Mtccamk/Mtdmi3 mock trans-zeatin) \pm SEM.

(D-F) Concentrations of the cytokinins isopentenyl adenine (iP) (D), isopentenyl adenine riboside (iPR) (E) and trans-zeatin (tZ) (F) in root susceptible zones of the $M$. truncatula wild type and Mtnsp1, Mtnsp2 and Mtnsp1Mtnsp2 mutants treated with mock or rhizobium LCOs $\left(10^{-9} \mathrm{M}, 3 \mathrm{~h}\right)$. Plants were grown on agar-solidified Fåhraeus medium containing $1 \mu \mathrm{M}$ AVG. Data represent the means of 5-6 biological replicates \pm SEM.

Different letters above the bars indicate a statistical difference $(p<0.05)$. Statistical significance was assessed based on ANOVA.

\section{Ethylene Inhibits Symbiotic Cytokinin Accumulation}

Previous work has shown that cytokinin can induce ethylene production [64-66]. Based on this, we hypothesized that LCO-induced cytokinin accumulation might also induce ethylene production. To test this hypothesis, we checked for ethylene biosynthesis genes among 

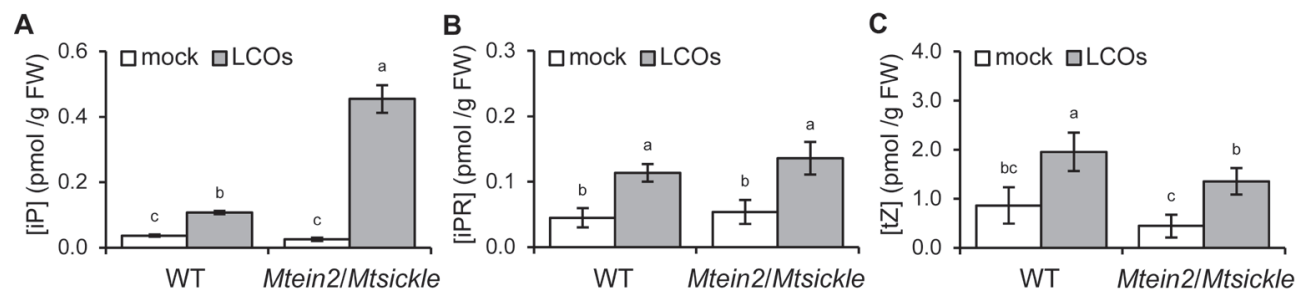

Figure 7. Ethylene negatively regulates LCO-induced cytokinin accumulation.

(A-C) Concentrations of the cytokinins isopentenyl adenine (iP) (A), isopentenyl adenine riboside (iPR) (B) and trans-zeatin (tZ) (C) in root susceptible zones of the $M$. truncatula wild type and Mtein2/ Mtsickle mutant treated with mock or rhizobium LCOs $\left(10^{-9} \mathrm{M}, 3 \mathrm{~h}\right)$. Plants were grown on agarsolidified Fåhraeus medium without AVG. Data represent the means of six biological replicates \pm SEM. Different letters above the bars indicate a statistical difference $(p<0.05)$. Statistical significance was assessed based on ANOVA.

the differentially expressed genes identified through RNA-seq. This revealed an MtCRE1dependent transcriptional induction of three ACS genes (Medtr8g101750, Medtr8g101820, Medtr4g097540) by rhizobium LCOs. However, close inspection of Medtr8g101820 revealed that this gene model either represents a pseudogene, or an error in the current genome assembly (Mt4.0; see Supplemental Figure 6). For the other two ACS genes, Medtr8g101750 (MtACS1) and Medtr4g097540 (MtACS2), transcriptional induction of by rhizobium LCOs is strongly supported (Figure $3 \mathrm{C}$ ). A detailed look at the expression of all putative ethylene biosynthesis genes revealed induction of an additional ACS gene (Medtr6g091760, MtACS3) just below the 2 -fold threshold ( $p<0.001, \mathrm{FC}=1.95$ ) (Figure $3 \mathrm{C}$ ).

qRT-PCR reactions on the root samples taken at different time points after LCO treatment, showed that all three ACS genes are induced already at 30 minutes after LCO exposure (Figure 4D). At three hours after LCO exposure, expression of all three ACS genes is induced at > 7-fold; however, we also observed dampening of expression at one hour after LCO treatment (Figure 4D). This may suggest a fast induction of ACS genes, which may occur in a cytokinin-independent fashion, followed by a strong MtCRE1-dependent induction at three hours after LCO exposure. Induction of MtACS1 occurs in both the presence and absence of AVG, although induction is much stronger if AVG is added to the growth medium ( $3.5-\mathrm{vs}$ $\sim 40$-fold) (Figure 5C). Expression of MtACS2 and MtACS3 is induced only in the presence of AVG, suggesting that induction of these genes requires low ethylene levels (Figure $\mathbf{5 C}$ ). These results indicate an MtCRE1-dependent transcriptional induction of at least one ethylene biosynthesis gene by rhizobium LCOs. This suggests that LCO-induced cytokinin accumulation promotes ethylene biosynthesis. 
Ethylene is a known inhibitor of rhizobium LCO signalling and subsequent nodule formation $[19,27]$. Therefore, cytokinin-dependent induction of ethylene biosynthesis genes by rhizobium LCOs may restrict LCO-induced cytokinin accumulation. The latter is supported by the effect of AVG on the responsiveness of cytokinin response regulators to rhizobium LCOs (Figure 5A). To determine the effect of ethylene on cytokinin accumulation, we exploited the $M$. truncatula ethylene insensitive mutant Mtein2/Mtsickle. To this end, $M$. truncatula wild type and Mtein2/Mtsickle plants were grown on medium without AVG and treated with rhizobium LCOs for three hours. Under these conditions, wild type accumulated 2- to 3-fold higher concentrations of iP, iPR and tZ after LCO treatment (Figure 7). This response seems weaker than compared with the accumulation detected in plants grown on regular plant growth medium that contains $1 \mu \mathrm{M}$ AVG (Figure 6A-C), indicating an inhibitory effect of ethylene. Cytokinin measurements in Mtein2/Mtsickle mutant also indicate negative regulation by ethylene. In this mutant, LCO treatment induced a 15 -fold increase in iP concentration, a response five times stronger compared with wild type (Figure 7A). Taken together, we conclude that rhizobium LCO-induced cytokinin accumulation is negatively affected by ethylene.

\section{Discussion}

Cytokinin signalling is an integral part of the signalling pathway leading to nodule organogenesis. However, whether cytokinin accumulates as a signalling intermediate and to what extent this is required for rhizobium LCO-induced signalling remained elusive. Here, we show that in $M$. truncatula, cytokinin rapidly accumulates in the root susceptible zone upon application of rhizobium LCOs. The accumulating cytokinins include iP and $t Z$, which are among the biologically most active cytokinins [41]. Furthermore, we show that the majority of transcriptional changes induced three hours after rhizobium LCO treatment are dependent on the cytokinin receptor, MtCRE1. Therefore, we conclude that cytokinin accumulation is a key step in the pathway leading to rhizobium root nodule organogenesis.

Using RNA-seq, we determined the extent of LCO-induced signalling dependent on MtCRE1. This revealed that the vast majority ( $73 \%$ ) of the transcriptional changes induced three hours after LCO application are dependent on this symbiotic cytokinin receptor. Genes that are differentially expressed in both wild type and Mtcre1 probably function upstream of MtCRE1 or in a cytokinin-independent pathway involved in rhizobial infection [67]. Examples of these are the cytokinin biosynthesis gene MtIPT1 and genes involved in chitin breakdown. The latter could be involved in removing excess rhizobium LCO signals or could be part of a general defence response induced by these bacterial signal molecules [68, 69]. In addition, 
some level of redundancy may contribute to the transcriptional changes observed in Mtcre1 upon rhizobium LCO exposure. The Mtcre1-1 mutant used in this study is considered a full knock-out as it has a mutation that creates a premature stop codon in the middle of the kinase domain [37]. It has been reported that in rare cases this mutant can develop a few nodules [37], even though we did not observe nodules on Mtcre1-1 mutant plants in any of our experiments (data not shown). In case of L. japonicus, a similar phenotype of the Ljlhk1-1 mutant could be explained by redundant functioning of the paralogous genes, LjLHK1A and $L$ LLHK3, indicating the existence of partial redundancy for symbiotic cytokinin perception [70]. However, in the $M$. truncatula Mtcre1-1 mutant redundancy by additional histidine receptors in symbiotic cytokinin signalling is most likely limited, as the expression of typical cytokinin responsive genes (e.g. the A-type Response Regulators) is not or only moderately induced in this mutant upon LCO application (Figure 5A and Supplemental Figure 1). Therefore, we conclude that most genes induced three hours after rhizobium LCO application function in the MtCRE1-dependent nodule organogenesis pathway.

Measurements of the cytokinin concentration in $M$. truncatula root susceptible zones showed accumulation of three cytokinins three hours after rhizobium LCO application. This response is dependent on rhizobium LCO signalling, as it was not observed in the Mtccamk/ Mtdmi3 mutant. Our observation that both iP and its biosynthetic precursor iPR accumulate upon rhizobium LCO exposure suggests that local biosynthesis is the primary source of these cytokinins. This is supported by induction of the cytokinin biosynthesis gene MtIPT1 at one hour after LCO exposure in both wild type and Mtcre1 mutant. However, considering the time frame in which cytokinin accumulates upon LCO signalling, we cannot exclude that the increase in cytokinin concentration is regulated primarily at the protein level.

We also determined the role of the GRAS-type transcriptional regulators, NSP1 and NSP2, in LCO-induced cytokinin accumulation and showed that iP and $\mathrm{tZ}$ accumulate to intermediate levels in both single and double mutants compared with wild type. Initially, it was argued that these GRAS-type proteins may act as primary rhizobium LCO response factors [71], for which indeed some experimental evidence was found [16, 72]. However, more in-depth genetic dissection of the rhizobium LCO signalling pathway suggests a more complex role for both proteins, downstream of as well as parallel to symbiotic cytokinin signalling [67]. Our finding shows that both NSP1 and NSP2 may function downstream of symbiotic cytokinin biosynthesis in an autoactivation loop. Such hypothesis is supported by the finding that MtNSP1 expression is induced three hours after LCO treatment and this induction is in part dependent on MtCRE1. 
We uncovered a negative feedback relation between cytokinin and ethylene. Cytokinin measurements showed that upon LCO signalling, the M. truncatula Mtein2/Mtsickle knockout mutant accumulates more cytokinin in the root susceptible zone than wild-type plants. This implies that ethylene signalling inhibits symbiotic cytokinin accumulation. This finding is in line with earlier studies that showed that ethylene interferes with LCO-induced calcium oscillations in and around the nuclei of activated cells $[19,73]$ and with the absence of cytokinin accumulation in the Mtccamk/Mtdmi3 mutant (Figure 6A-C). Our data also indicate a cytokinin-dependent transcriptional induction of (at least) one ACC synthase by rhizobium LCOs. This is in line with results on L. japonicus, which show that the ethylene-producing ACC oxidase $L j A C O 2$ is also induced by rhizobium LCOs [74]. Furthermore, previous work has shown that cytokinin can induce ethylene production [64-66]. Therefore, it is conceivable that LCO signalling, through an induction of cytokinin accumulation, might induce ethylene biosynthesis. This response could be part of a negative feedback signal to inhibit further LCO signalling and subsequent cytokinin accumulation (Figure 8). Such a feedback mechanism might be important to restrict LCO-induced cytokinin accumulation to a limited number of cells to prevent initiation of multiple nodule primordia, as can be observed in the Mtein2/ Mtsickle mutant. This response would resemble the mechanism through which nodule positioning is regulated, where ethylene biosynthesis genes are expressed opposite phloem poles, delineating nodule initiation [22]. In addition, cytokinin-induced ethylene production might be required to restrict rhizobial infections. The latter is suggested by the phenotype of the L. japonicus Ljlhk1 mutant, which displays excessive infection thread formation upon rhizobial inoculation [18]. This phenotype suggests the absence of a negative feedback signal to inhibit rhizobium LCO-induced signalling. It is conceivable that this signal would be ethylene.

Measurements of the cytokinin concentration in wild-type $M$. truncatula showed that both iP and tZ accumulate after LCO exposure. Interestingly, accumulation of iP after LCO treatment was elevated in the Mtein2/Mtsickle mutant compared to wild type, whereas accumulation of $\mathrm{tZ}$ was comparable for both genotypes. This suggests that LCO-induced accumulation of iP is more sensitive to negative regulation by ethylene, as compared to accumulation of $\mathrm{tZ}$. However, the mechanistic basis for this difference is currently unclear. Sensitivity to different cytokinins differs between individual cytokinin receptors $[75,76]$. The arabidopsis AtAHK4/AtCRE1 cytokinin receptor possesses similar sensitivity to both iP and tZ $[75,76]$. AtAHK4/MtCRE1 represents that closest orthologue of MtCRE1 and LjLHK1 and was shown to functionally complement the nodulation defect of the Ljlhk1 mutant [35-37, 70]. Therefore, it seems plausible that MtCRE1 and LjLHK1 also display equal sensitivity to iP and $t Z$, leaving the individual contributions of these cytokinins to nodulation undetermined. 


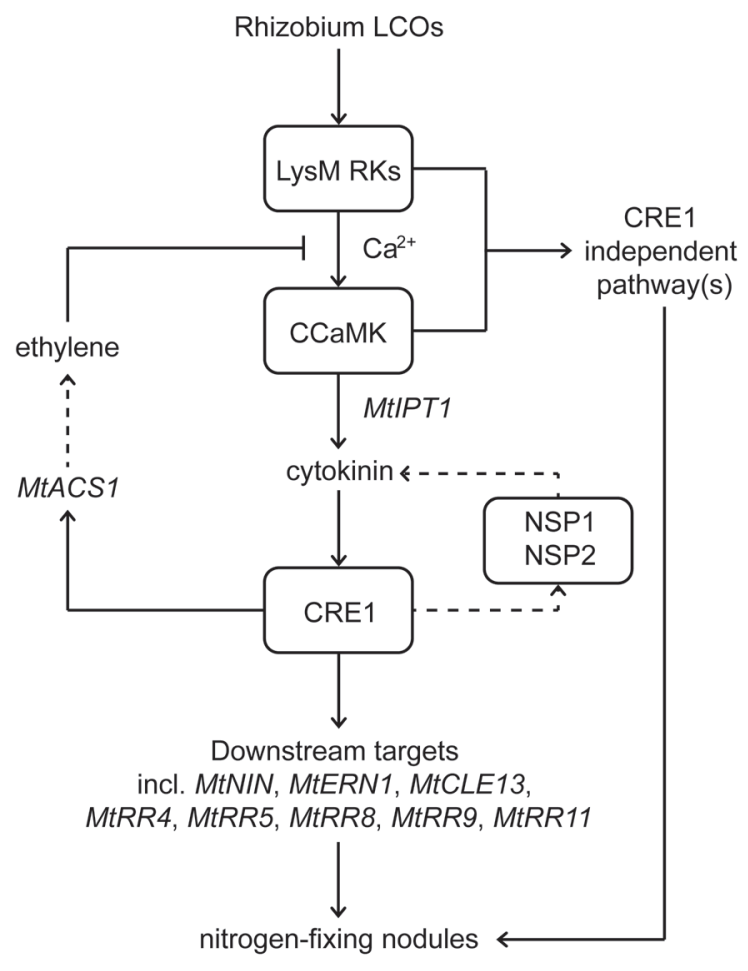

Figure 8. Proposed model for the position of cytokinin and ethylene in the rhizobium LCO signalling pathway.

Recent work on L. japonicus showed that cytokinins might function not only to induce nodulation but also to suppress nodule formation as part of the autoregulation of nodulation (AON) [77]. Activation of CLE-RS1/2-HAR1 signalling increased levels of the iPRP cytokinin precursors in shoots, suggesting a role for cytokinins in AON. Increased iPRP levels in shoots was linked to an induction of LJIPT3 in shoots at three days after rhizobium inoculation [77]. This response is much slower than the accumulation of cytokinins that we observed, which occurred at three hours after LCO application. Therefore, it is highly unlikely that the increase in cytokinin concentrations that we observed is caused by activation of AON. Furthermore, Sasaki et al. [77] did not report increased levels of cytokinin in roots after activation of AON. Therefore, it remains undetermined whether cytokinins function in roots to repress nodule formation upon activation of AON. Cytokinin as part of AON seems to function upstream of TML, a Kelch-repeat containing F-box protein [77]. TML appears to inhibit nodule formation downstream of cytokinin signalling, as spontaneous nodule formation induced by snf2, a 
gain-of-function allele of the cytokinin receptor $L$ LHKK1, is also suppressed by TML [78]. Identification of the targets of TML might show if and how cytokinins might function to both induce and suppress nodule initiation.

Nodule primordium formation results from mitotic activation of root cortical and pericycle cells and is associated with the formation of a local auxin maximum [79-83]. Mathematical modelling revealed that creation of such an auxin maximum most likely results from a reduction in auxin transport [84]. In arabidopsis, cytokinin has been shown to affect auxin transport by targeting the PIN auxin efflux carriers at both the gene expression and protein level [85-87]. Also in legumes, cytokinin seems to affect auxin transport. In Mtcre1 mutant roots, expression of several PIN genes is affected and the polar auxin transport rate is increased [37]. Furthermore, reduction of the polar auxin transport rate observed upon inoculation with rhizobia does not occur in Mtcre1 mutant roots [37]. Based on this, it is hypothesized that cytokinin is the signal that induces auxin accumulation in the root cortex and pericycle upon epidermal perception of rhizobium LCOs [88]. Rapid accumulation of cytokinin and induction of cytokinin signalling in the root cortex, endodermis and pericycle, as observed by TCS:GUS expression, suggests that cytokinin functions non-cell-autonomously. We did not observe activation of TCS:GUS expression in the root epidermis after rhizobium LCO treatment. This would suggest that cytokinin signalling and possibly cytokinin biosynthesis take place in the root cortex. However, studies on cytokinin response regulators did show activation of cytokinin responses in the root epidermis after rhizobium LCO-induced signalling $[36,58,89]$. A recent study on redundant function of cytokinin receptors in $L$. japonicus also suggests a role for epidermal cytokinin signalling in root nodule initiation [70]. The authors show that cytokinin receptors LjLHK1A and LjLHK3 can partially rescue the Ljlhk1 mutant phenotype. However, in this case nodule formation is only initiated after rhizobium bacteria reach the root cortex. As only LjLHK1 is expressed in the root epidermis, this suggests that epidermal cytokinin signalling is required for nodule formation [70]. This is consistent with a function for cytokinin as systemic signal and suggests that cytokinin might bridge the gap between epidermal perception of rhizobium LCOs and initiation of nodule primordia in the interior cell layers of the legume root.

Taken together, we have demonstrated that rhizobium LCOs cause an increase in the abundance of various cytokinins prior to the first mitotic divisions. It is now a challenge to unveil the molecular mechanism behind the accumulation of this hormone. This will provide insight into how existing developmental modules have been co-opted during the evolution of legume-rhizobium root nodule symbiosis. 


\section{Methods}

\section{Plant Materials and Treatments}

Medicago truncatula Jemalong A17, Mtccamk/Mtdmi3 (TRV25) [90], Mtnsp1-1 (B85) [71, 90], Mtnsp2-2 (0-4) [91, 92], Mtnsp1Mtnsp2 [93], Mtcre1-1 [37] and Mtein2/Mtsickle-1 mutant [94] seedlings were grown vertically for three days on modified Fåhraeus medium agar plates with low nitrate (including $0.2 \mathrm{mM} \mathrm{Ca}\left(\mathrm{NO}_{3}\right)_{2}$ ) supplemented with $1 \mu \mathrm{M} \mathrm{AVG}$, unless stated otherwise [95]. Then, Sinorhizobium meliloti 2011 Nod factors $\left(\sim 10^{-9} \mathrm{M}\right)$ or water as a control was pipetted on top of the root susceptible zone. Roots were exposed for three hours and subsequently $1-\mathrm{cm}$ root pieces were cut just above the root tip and snapfrozen in liquid nitrogen ( $\sim n=50$ for cytokinin extractions, $\sim n=15$ for RNA isolation). For all experiments, plants were grown in an environmentally controlled growth chamber at $20^{\circ} \mathrm{C}$ with a $16-\mathrm{h}$ light/8-h dark cycle and $70 \%$ relative humidity.

\section{Vectors and Constructs}

The synthetic cytokinin reporter element TCS was recombined into a pENTR-D-Topo (Invitrogen, Carlsbad, USA) thereby creating pENTR1-2_TCS [62]. Subsequently, this construct was recombined into pKGWFS7-RR containing a GUS-GFP fusion reporter by a gateway reaction (Invitrogen, Carlsbad, USA). All binary destination vectors used in this study contain AtUBQ10 ${ }_{\text {pro }}$ :DsRed1 as selectable marker [97]. All cloning vectors and constructs are available upon request from our laboratory or via the Functional Genomics unit of the Department of Plant Systems Biology (VIB-Ghent University).

\section{Plant Transformation and Histology}

Agrobacterium rhizogenes-mediated root transformation was used to transform $M$. truncatula (Jemalong A17) as described by Limpens et al. [98]. Transgenic roots were selected based on DsRED1 expression. Transgenic roots were transferred to low nitrate Fåhraeus plates (including $0.2 \mathrm{mM} \mathrm{Ca}_{2}\left(\mathrm{NO}_{3}\right)_{2}$ ) supplemented with $1 \mu \mathrm{M}$ AVG three weeks after transformation. After five days on these plates, Sinorhizobium meliloti 2011 Nod factors $\left(\sim 10^{-9} \mathrm{M}\right)$ or water as a control was pipetted on top of the root zone susceptible to rhizobium infection. Roots were GUS stained three hours after treatment and fixed as described in Limpens et al. [99]. Microtome sections of $9 \mu \mathrm{m}$ were stained with ruthenium red and photographed using a Leica DM5500B microscope equipped with a DFC425C camera (Leica Microsystems, Wetzlar, Germany). Images were digitally processed using Photoshop CS6 (Adobe Systems, San Jose, USA). 


\section{RNA Sequencing}

RNA was isolated from snap-frozen roots samples using the plant RNA kit (E.Z.N.A, Omega Biotek, Norcross, USA) as described in the manufacturer's protocol. RNA was sequenced at BGI Tech Solutions (Beijing Genomics Institute, Hong Kong, China) using the Illumina Truseq (Transcriptome) protocol utilizing an Illumina Hiseq 2000 instrument. This generated 90-bp paired-end reads with an average insert size of 130-160 bp. In total 24-26 million clean reads were generated for each sample (Supplemental Table 8). Sequencing data were analysed by mapping RNA-seq reads against the $M$. truncatula genome (Mt4.0) using the RNA-seq module implemented in CLC Genomics Workbench v7.5 (CLC bio, Aarhus, Denmark) with default settings, with the exception of the similarity fraction, which was changed to 0.95 . As such, $>95 \%$ of the reads generated for each sample were successfully mapped against the M. truncatula genome (Mt4.0), of which > 90\% mapped in pairs (Supplemental Table 8). Differentially expressed genes were selected based on a minimal fold change of 2 and a Bonferroni-corrected $p$-value $<0.001$, as determined using the EdgeR test implemented in CLC [100]. GO enrichment analysis was performed using the Hypergeometric tests on annotations method implemented in CLC.

\section{Quantitative RT-PCR}

RNA was isolated from snap-frozen roots samples using the plant RNA kit (E.Z.N.A, Omega Biotek, Norcross, USA) as described in the manufacturer's protocol. cDNA was synthesized from $1 \mu \mathrm{g}$ total RNA using an i-script cDNA synthesis kit (Bio-Rad, Hercules, USA) as described in the manufacturer's protocol. Real time qRT-PCR was set up in a 10- $\mu$ l reaction system with 2x iQ SYBR Green Super-mix (Bio-Rad, Hercules, USA). The experimental setup and procedure were executed using a CFX Connect optical cycler, according to the protocol provided by the manufacturer (Bio-Rad, Hercules, USA). All primers including the genes used for normalization (MtUBQ10 and MtPTB) are given in Supplemental Table 9. Data analysis was performed using CFX Manager 3.0 software (Bio-Rad, Hercules, USA). Cq values of 32 and higher were excluded from the analysis, though still checked for transcriptional induction (see Supplemental Table 9). Statistical significance was determined based on Student's $t$-test $(p<0.01)$, as implemented in CFX Manager 3.0 software (Bio-Rad, Hercules, USA).

\section{Cytokinin Extraction}

For extraction of cytokinins from M. truncatula root material, 100 mg of snap-frozen plant material (root susceptible zones) was used. Tissue was ground to a fine powder using 3-mm stainless steel beads at $50 \mathrm{~Hz}$ for $1 \mathrm{~min}$ in a Tissuelyser LT (Qiagen, Germantown, USA). Ground root samples were extracted with $1 \mathrm{ml}$ of $100 \%$ methanol $(\mathrm{MeOH})$ containing 2.5 
$\mathrm{mM}$ of diethyldithiocarbamic acid and stable isotope-labelled cytokinin internal standards (IS, Supplemental Table 10 numbers 1-7) were used at a concentration of 100 pmol per compound per sample. Samples were extracted by short vortexing and ultrasonication during $10 \mathrm{~min}$. Subsequently, samples were centrifuged at $2000 \mathrm{rpm}$ for $10 \mathrm{~min}$ in a tabletop centrifuge at room temperature (RT). Supernatants were transferred to $4 \mathrm{ml}$ glass vials. Pellets were re-extracted with $1 \mathrm{ml} 100 \% \mathrm{MeOH}$ for one hour on a shaker at $4^{\circ} \mathrm{C}$. After centrifugation as above, both supernatants were pooled and $\mathrm{MeOH}$ evaporated in a speed vacuum system (SPD121P, Thermo Savant, Hastings, UK) at RT. Residues were resuspended in $50 \mu \mathrm{l} \mathrm{MeOH}$ and then diluted in $3 \mathrm{ml}$ of water before loading on a $50 \mathrm{mg}$ GracePure SPE C18-max cartridge (Grace, Columbia, USA). The cartridge was equilibrated with $2 \mathrm{ml}$ of water prior to sample loading. Subsequently, the cartridge was washed with $1 \mathrm{ml}$ of water and eluted with $1 \mathrm{ml}$ of $100 \%$ acetone. The acetone was evaporated in a speed vacuum system at RT and the residue resuspended in $200 \mu \mathrm{l}$ acetonitrile/water/formic acid (10:90:0.1, v/v/v). The sample was filtered through a 0.45- $\mu \mathrm{m}$ Minisart SRP4 filter (Sartorius, Goettingen, Germany) and stored at $-20^{\circ} \mathrm{C}$.

\section{Cytokinin Detection and Quantification by Liquid Chromatography-Tandem Mass Spectrometry}

Analyses of cytokinins in $M$. truncatula root extracts were performed by comparing retention times and mass transitions with those of cytokinin standards (Supplemental Table 10) using a Waters Xevo TQ mass spectrometer equipped with an electrospray ionization source coupled to an Acquity UPLC system (Waters, Milford, USA) using settings as previously described $[101,102]$. Chromatographic separations have been conducted on an Acquity UPLC BEH C18 column (100 mm, $2.1 \mathrm{~mm}, 1.7 \mathrm{~mm}$; Waters, USA) by applying a water/acetonitrile gradient. The water/acetonitrile gradient started from $0.2 \%(\mathrm{v} / \mathrm{v})$ of acetonitrile for $1.5 \mathrm{~min}$ increasing to $20 \%(\mathrm{v} / \mathrm{v})$ of acetonitrile in $8.5 \mathrm{~min}$. To wash the column, the water/acetonitrile gradient was increased to $70 \%(\mathrm{v} / \mathrm{v})$ acetonitrile in a 1.0 -min gradient, which was maintained for 0.7 min before going back to $0.2 \%$ acetonitrile using a 0.3 -min gradient, prior to the next run. Finally, the column was equilibrated for 2.5 min using this solvent composition. The column was operated at $50^{\circ} \mathrm{C}$ with a flow rate of $0.6 \mathrm{ml} \mathrm{min}^{-1}$. The sample injection volume was 20 $\mu \mathrm{l}$. The mass spectrometer was operated in positive electrospray ionization mode. Cone and desolvation gas flows were set to 50 and $1000 \mathrm{I} \mathrm{h}^{-1}$, respectively. The capillary voltage was set at $3.0 \mathrm{kV}$, the source temperature at $150^{\circ} \mathrm{C}$ and the desolvation temperature at $650^{\circ} \mathrm{C}$. The cone voltage was optimized for each standard compound using the IntelliStart MS Console (Waters, Milford, USA). Argon was used for fragmentation by collision induced dissociation (CID). Multiple reaction monitoring (MRM) was used for quantification. Parent- 
daughter transitions for the different cytokinins and deuterium-labelled cytokinins were set using the IntelliStart MS Console. MRM transitions selected for cytokinin identification and quantification are shown in Supplemental Table 10. Cone voltage was set to $18 \mathrm{eV}$.

Cytokinins were quantified using a calibration curve with known amount of standards and based on the ratio of the peak areas of the MRM transition for standards to the MRM transition for the corresponding deuterium-labelled cytokinins (Supplemental Table 10 numbers 1-7). Data acquisition and analysis were performed using MassLynx 4.1 (TargetLynx) software (Waters, Milford, USA). The summed area of all corresponding MRM transitions was used for statistical analysis.

\section{Accession Numbers}

RNA-seq reads have been deposited in the Array-Express (http://www.ebi.ac.uk/ arrayexpress) database under accession number E-MTAB-3007.

\section{SUPPLEMENTAL DATA}

Supplemental data belonging to this chapter are available at Molecular Plant Online (https:// doi.org/10.1016/j.molp.2015.03.010).

\section{ACKNOWLEDGMENTS}

We thank Bruno Müller for providing the TCS reporter, Florian Frugier for Mtcre1-1 seeds, and Robin van Velzen for help with RNA-seq analysis. This work was supported by European Research Council (ERC-2011-AdG294790), NWO-NSFC Joined Research project (846.11.005) and NWO VICl (865.13.001). 


\section{References}

1 Crespi, M. and Frugier, F. (2008) De novo organ formation from differentiated cells: root nodule organogenesis. Sci. Signal. 1, re11

2 Kouchi, H. et al. (2010) How many peas in a pod? Legume genes responsible for mutualistic symbioses underground. Plant Cell Physiol. 51, 1381-1397

3 Murray, J.D. (2011) Invasion by invitation: Rhizobial infection in legumes. Mol. Plant. Microbe Interact. 24, 631-639

4 Yokota, K. and Hayashi, M. (2011) Function and evolution of nodulation genes in legumes. Cell. Mol. Life Sci. $68,1341-1351$

5 Lerouge, P. et al. (1990) Symbiotic host-specificity of Rhizobium meliloti is determined by a sulphated and acylated glucosamine oligosaccharide signal. Nature 344, 781-784

6 Mergaert, P. et al. (1993) Three unusual modifications, a D-arabinosyl, an N-methyl, and a carbamoyl group, are present on the Nod factors of Azorhizobium caulinodans strain ORS571. Proc. Natl. Acad. Sci. U. S. A. 90, 1551-1555

7 Stokkermans, T.J. and Peters, N.K. (1994) Bradyrhizobium elkanii lipo-oligosaccharide signals induce complete nodule structures on Glycine soja Siebold et Zucc. Planta 193, 413-420

8 Relić, B. et al. (1994) Nod factors of Rhizobium are a key to the legume door. Mol. Microbiol. 13, 171-179

9 Truchet, G. et al. (1991) Sulphated lipo-oligosaccharide signals of Rhizobium meliloti elicit root nodule organogenesis in alfalfa. Nature 351, 670-673

10 Limpens, E. et al. (2003) LysM domain receptor kinases regulating rhizobial Nod factor-induced infection. Science 302, 630-633

11 Arrighi, J.F. et al. (2006) The Medicago truncatula lysine motif-receptor-like kinase gene family includes NFP and new nodule-expressed genes. Plant Physiol. 142, 265-279

12 Radutoiu, S. et al. (2003) Plant recognition of symbiotic bacteria requires two LysM receptor-like kinases. Nature 425, 585-592

13 Radutoiu, S. et al. (2007) LysM domains mediate lipochitin-oligosaccharide recognition and $N f r$ genes extend the symbiotic host range. $Е M B O$ J. 26, 3923-3935

14 Oldroyd, G.E.D. (2013) Speak, friend, and enter: signalling systems that promote beneficial symbiotic associations in plants. Nature Rev. Microbiol. 11, 252-263

15 Levy, J. et al. (2004) A putative $\mathrm{Ca}^{2+}$ and calmodulin-dependent protein kinase required for bacterial and fungal symbioses. Science 303, 1361-1364

16 Mitra, R.M. et al. (2004) A Ca ${ }^{2+} /$ calmodulin-dependent protein kinase required for symbiotic nodule development: Gene identification by transcript-based cloning. Proc. Natl. Acad. Sci. U. S. A. 101, 4701-4705

17 Singh, S. et al. (2014) CYCLOPS, a DNA-binding transcriptional activator, orchestrates symbiotic root nodule development. Cell Host Microbe 15, 139-152

18 Murray, J.D. et al. (2007) A cytokinin perception mutant colonized by Rhizobium in the absence of nodule organogenesis. Science 315, 101-104

19 Oldroyd, G.E.D. et al. (2001) Ethylene inhibits the Nod factor signal transduction pathway of Medicago truncatula. Plant Cell 13, 1835-1849

20 Tirichine, L. et al. (2007) A gain-of-function mutation in a cytokinin receptor triggers spontaneous root nodule organogenesis. Science 315, 104-107

21 Lin, Z. et al. (2009) Recent advances in ethylene research. J. Exp. Bot. 60, 3311-3336

22 Heidstra, R. et al. (1997) Ethylene provides positional information on cortical cell division but is not involved in Nod factor-induced root hair tip growth in rhizobium-legume interaction. Development 124, 1781-1787

23 Roman, G. et al. (1995) Genetic analysis of ethylene signal transduction in Arabidopsis thaliana: five novel mutant loci integrated into a stress response pathway. Genetics 139, 1393-1409

24 Alonso, J.M. et al. (1999) EIN2, a bifunctional transducer of ethylene and stress responses in Arabidopsis. Science 284, 2148-2152

25 Merchante, C. et al. (2013) Ethylene signaling: Simple ligand, complex regulation. Curr. Opin. Plant Biol. 16, 554-560

26 Penmetsa, R.V. et al. (2003) Dual genetic pathways controlling nodule number in Medicago truncatula. Plant 
Physiol. 131, 998-1008

27 Penmetsa, R.V. et al. (2008) The Medicago truncatula ortholog of Arabidopsis EIN2, sickle, is a negative regulator of symbiotic and pathogenic microbial associations. Plant J. 55, 580-595

28 Torrey, J.G. (1961) Kinetin as trigger for mitosis in mature endomitotic plant cells. Exp. Cell Res. 23, 281-299

29 Cooper, J.B. and Long, S.R. (1994) Morphogenetic rescue of Rhizobium meliloti nodulation mutants by transzeatin secretion. Plant Cell 6, 215-225

30 Mathesius, U. et al. (2000) Temporal and spatial order of events during the induction of cortical cell divisions in white clover by Rhizobium leguminosarum bv. trifolii inoculation or localized cytokinin addition. Mol. Plant. Microbe Interact. 13, 617-628

31 Heckmann, A.B. et al. (2011) Cytokinin induction of root nodule primordia in Lotus japonicus is regulated by a mechanism operating in the root cortex. Mol. Plant. Microbe Interact. 24, 1385-1395

32 Franco-Zorrilla, J.M. et al. (2002) Mutations at CRE1 impair cytokinin-induced repression of phosphate starvation responses in Arabidopsis. Plant J. 32, 353-360

33 de León, B.G.-P. et al. (2004) Interallelic complementation at the Arabidopsis CRE1 locus uncovers independent pathways for the proliferation of vascular initials and canonical cytokinin signalling. Plant J. 38, 70-79

34 Higuchi, M. et al. (2004) In planta functions of the Arabidopsis cytokinin receptor family. Proc. Natl. Acad. Sci. U. S. A. $101,8821-8826$

35 Gonzalez-Rizzo, S. et al. (2006) The Medicago truncatula CRE1 cytokinin receptor regulates lateral root development and early symbiotic interaction with Sinorhizobium meliloti. Plant Cell 18, 2680-2693

36 Op den Camp, R.H. et al. (2011) A phylogenetic strategy based on a legume-specific whole genome duplication yields symbiotic cytokinin type-A response regulators. Plant Physiol. 157, 2013-2022

37 Plet, J. et al. (2011) MtCRE1-dependent cytokinin signaling integrates bacterial and plant cues to coordinate symbiotic nodule organogenesis in Medicago truncatula. Plant J. 65, 622-633

38 Ovchinnikova, E. et al. (2011) IPD3 controls the formation of nitrogen-fixing symbiosomes in pea and Medicago spp. Mol. Plant. Microbe Interact. 24, 1333-1344

39 Vernie, T. et al. (2008) EFD is an ERF transcription factor involved in the control of nodule number and differentiation in Medicago truncatula. Plant Cell 20, 2696-2713

40 Sakakibara, H. (2006) Cytokinins: Activity, biosynthesis, and translocation. Annu. Rev. Plant Biol. 57, 431-449

41 Kamada-Nobusada, T. and Sakakibara, H. (2009) Molecular basis for cytokinin biosynthesis. Phytochemistry 70, 444-449

42 Takei, K. et al. (2004) Arabidopsis CYP735A1 and CYP735A2 encode cytokinin hydroxylases that catalyze the biosynthesis of trans-zeatin. J. Biol. Chem. 279, 41866-41872

43 Kurakawa, T. et al. (2007) Direct control of shoot meristem activity by a cytokinin-activating enzyme. Nature $445,652-655$

44 Kuroha, T. et al. (2009) Functional analyses of LONELY GUY cytokinin-activating enzymes reveal the importance of the direct activation pathway in Arabidopsis. Plant Cell 21, 3152-3169

45 Schmülling, T. et al. (2003) Structure and function of cytokinin oxidase/dehydrogenase genes of maize, rice, Arabidopsis and other species. J. Plant Res. 116, 241-252

46 Zhang, X. et al. (2013) Adenine phosphoribosyl transferase 1 is a key enzyme catalyzing cytokinin conversion from nucleobases to nucleotides in Arabidopsis. Mol. Plant 6, 1661-1672

47 Chen, Y. et al. (2014) Knockdown of LjIPT3 influences nodule development in Lotus japonicus. Plant Cell Physiol. 55, 183-193

48 Mortier, V. et al. (2014) Role of LONELY GUY genes in indeterminate nodulation on Medicago truncatula. New Phytol. 202, 582-593

49 Haney, C.H. et al. (2011) Symbiotic rhizobia bacteria trigger a change in localization and dynamics of the Medicago truncatula receptor kinase LYK3. Plant Cell 23, 2774-2787

50 Horváth, B. et al. (2011) Medicago truncatula IPD3 is a member of the common symbiotic signaling pathway required for rhizobial and mycorrhizal symbioses. Mol. Plant. Microbe Interact. 24, 1345-1358

51 Domonkos, A. et al. (2013) The identification of novel loci required for appropriate nodule development in Medicago truncatula. BMC Plant Biol. 13, 157

52 Gourion, B. et al. (2013) Protocols for growing plant symbioses; rhizobia. In Plant Mineral Nutrients: Methods and Protocols, Methods in Molecular Biology (Maathuis, F.J.M., ed), pp. 61-75

53 Berrabah, F. et al. (2014) Growth conditions determine the DNF2 requirement for symbiosis. PLoS One 9, 
e91866

54 Xiao, T.T. et al. (2014) Fate map of Medicago truncatula root nodules. Development 141, 3517-3528

55 Bianco, M.D. et al. (2013) Spatiotemporal changes in the role of cytokinin during root development. New Phytol. 199, 324-338

56 Journet, E.P. et al. (2001) Medicago truncatula ENOD11: a novel RPRP-encoding early nodulin gene expressed during mycorrhization in arbuscule-containing cells. Mol. Plant. Microbe Interact. 14, 737-748

57 Mortier, V. et al. (2010) CLE peptides control Medicago truncatula nodulation locally and systemically. Plant Physiol. 153, 222-237

58 Ariel, F. et al. (2012) Two direct targets of cytokinin signaling regulate symbiotic nodulation in Medicago truncatula. Plant Cell 24, 3838-3852

59 Benedito, V.A. et al. (2008) A gene expression atlas of the model legume Medicago truncatula. Plant J. 55, 504-513

60 Czaja, L.F. et al. (2012) Transcriptional responses toward diffusible signals from symbiotic microbes reveal MtNFP- and MtDMI3-dependent reprogramming of host gene expression by arbuscular mycorrhizal fungal lipochitooligosaccharides. Plant Physiol. 159, 1671-1685

61 Breakspear, A. et al. (2014) The root hair "infectome" of Medicago truncatula uncovers changes in cell cycle genes and reveals a requirement for auxin signaling in rhizobial infection. Plant Cell 26, 4680-4701

62 Muller, B. and Sheen, J. (2008) Cytokinin and auxin interaction in root stem-cell specification during early embryogenesis. Nature 453, 1094-1097

63 Bielach, A. et al. (2012) Spatiotemporal regulation of lateral root organogenesis in Arabidopsis by cytokinin. Plant Cell 24, 3967-3981

64 Fuchs, Y. and Lieberman, M. (1968) Effects of kinetin, IAA, and gibberellin on ethylene production, and their interactions in growth of seedlings. Plant Physiol. 43, 2029-2036

65 Lorteau, M.A. et al. (2001) Effects of cytokinin on ethylene production and nodulation in pea (Pisum sativum) cv. Sparkle. Physiol. Plant. 112, 421-428

66 Chae, H.S. et al. (2003) The eto1, eto2, and eto3 mutations and cytokinin treatment increase ethylene biosynthesis in Arabidopsis by increasing the stability of ACS protein. Plant Cell 15, 545-559

67 Madsen, L.H. et al. (2010) The molecular network governing nodule organogenesis and infection in the model legume Lotus japonicus. Nat. Commun. 1, 10

68 Nakagawa, T. et al. (2011) From defense to symbiosis: limited alterations in the kinase domain of LysM receptor-like kinases are crucial for evolution of legume-Rhizobium symbiosis. Plant J. 65, 169-180

69 Serna-Sanz, A. et al. (2011) Phosphoproteome analysis of Lotus japonicus roots reveals shared and distinct components of symbiosis and defense. Mol. Plant. Microbe Interact. 24, 932-937

70 Held, M. et al. (2014) Lotus japonicus cytokinin receptors work partially redundantly to mediate nodule formation. Plant Cell 26, 678-694

71 Smit, P. et al. (2005) NSP1 of the GRAS protein family is essential for rhizobial Nod factor-induced transcription. Science 308, 1789-1791

72 Hirsch, S. et al. (2009) GRAS proteins form a DNA binding complex to induce gene expression during nodulation signaling in Medicago truncatula. Plant Cell 21, 545-557

73 Oldroyd, G.E.D. et al. (2001) Evidence for structurally specific negative feedback in the Nod factor signal transduction pathway. Plant J. 28, 191-199

74 Miyata, K. et al. (2013) Two distinct EIN2 genes cooperatively regulate ethylene signaling in Lotus japonicus. Plant Cell Physiol. 54, 1469-1477

75 Stolz, A. et al. (2011) The specificity of cytokinin signalling in Arabidopsis thaliana is mediated by differing ligand affinities and expression profiles of the receptors. Plant J. 67, 157-168

76 Lomin, S.N. et al. (2015) Plant membrane assays with cytokinin receptors underpin the unique role of free cytokinin bases as biologically active ligands. J. Exp. Bot. 66, 1851-1863

77 Sasaki, T. et al. (2014) Shoot-derived cytokinins systemically regulate root nodulation. Nat. Commun. 5, 4983

78 Takahara, M. et al. (2013) TOO MUCH LOVE, a novel Kelch repeat-containing F-box protein, functions in the long-distance regulation of the legume-Rhizobium symbiosis. Plant Cell Physiol. 54, 433-447

79 Mathesius, U. et al. (1998) Auxin transport inhibition precedes root nodule formation in white clover roots and is regulated by flavonoids and derivatives of chitin oligosaccharides. Plant J. 14, 23-34

80 Pacios-Bras, C. et al. (2003) Auxin distribution in Lotus japonicus during root nodule development. Plant Mol. 
Biol. 52, 1169-1180

81 Huo, X. et al. (2006) RNAi phenotypes and the localization of a protein::GUS fusion imply a role for Medicago truncatula PIN genes in nodulation. J. Plant Growth Regul. 25, 156-165

82 van Noorden, G.E. et al. (2007) Overlap of proteome changes in Medicago truncatula in response to auxin and Sinorhizobium meliloti. Plant Physiol. 144, 1115-1131

83 Takanashi, K. et al. (2011) Involvement of auxin distribution in root nodule development of Lotus japonicus. Planta 234, 73-81

84 Deinum, E.E. et al. (2012) Modeling a cortical auxin maximum for nodulation: Different signatures of potential strategies. Front. Plant Sci. 3, 96

85 Dello loio, R. et al. (2008) A genetic framework for the control of cell division and differentiation in the root meristem. Science 322, 1380-1384

86 Marhavy, P. et al. (2011) Cytokinin modulates endocytic trafficking of PIN1 auxin efflux carrier to control plant organogenesis. Dev. Cell 21, 796-804

87 Marhavy, P. et al. (2014) Cytokinin controls polarity of PIN1-dependent auxin transport during lateral root organogenesis. Curr. Biol. 24, 1031-1037

88 Suzaki, T. et al. (2013) Genetic basis of cytokinin and auxin functions during root nodule development. Front. Plant Sci. 4, 42

89 Lohar, D.P. et al. (2004) Cytokinins play opposite roles in lateral root formation, and nematode and Rhizobial symbioses. Plant J. 38, 203-214

90 Catoira, R. et al. (2000) Four genes of Medicago truncatula controlling components of a nod factor transduction pathway. Plant Cell 12, 1647-1666

91 Oldroyd, G.E.D. and Long, S.R. (2003) Identification and characterization of Nodulation-Signaling Pathway 2, a gene of Medicago truncatula involved in Nod factor signaling. Plant Physiol. 131, 1027-1032

92 Kalo, P. et al. (2005) Nodulation signaling in legumes requires NSP2, a member of the GRAS family of transcriptional regulators. Science 308, 1786-1789

93 Liu, W. et al. (2011) Strigolactone biosynthesis in Medicago truncatula and rice requires the symbiotic GRAStype transcription factors NSP1 and NSP2. Plant Cell 23, 3853-3865

94 Penmetsa, R.V. and Cook, D.R. (1997) A legume ethylene-insensitive mutant hyperinfected by its rhizobial symbiont. Science $275,527-530$

95 Fåhraeus, G. (1957) The infection of clover root hairs by nodule bacteria studied by a simple glass slide technique. J. Gen. Microbiol. 16, 374-381

96 Ulmasov, T. et al. (1997) Aux/IAA proteins repress expression of reporter genes containing natural and highly active synthetic auxin response elements. Plant Cell 9, 1963-1971

97 Karimi, M. et al. (2002) GATEWAY' vectors for Agrobacterium-mediated plant transformation. Trends Plant Sci. 7, 193-195

98 Limpens, E. et al. (2004) RNA interference in Agrobacterium rhizogenes-transformed roots of Arabidopsis and Medicago truncatula. J. Exp. Bot. 55, 983-992

99 Limpens, E. et al. (2005) Formation of organelle-like $\mathrm{N}_{2}$-fixing symbiosomes in legume root nodules is controlled by DMI2. Proc. Natl. Acad. Sci. U. S. A. 102, 10375-10380

100 Robinson, M.D. et al. (2010) edgeR: a Bioconductor package for differential expression analysis of digital gene expression data. Bioinformatics 26, 139-140

101 Kohlen, W. et al. (2011) Strigolactones are transported through the xylem and play a key role in shoot architectural response to phosphate deficiency in nonarbuscular mycorrhizal host Arabidopsis. Plant Physiol. $155,974-987$

102 Kohlen, W. et al. (2012) The tomato CAROTENOID CLEAVAGE DIOXYGENASE8 (SICCD8) regulates rhizosphere signaling, plant architecture and affects reproductive development through strigolactone biosynthesis. New Phytol. 196, 535-547 



\section{Chapter 4}

\section{The strigolactone biosynthesis gene DWARF27 is co-opted in rhizobium symbiosis}

Arjan van Zeij ${ }^{*}$, Wei Liu*, Ting Ting Xiao*, Wouter Kohlen, Wei-Cai Yang, Ton Bisseling and Rene Geurts (2015) The strigolactone biosynthesis gene DWARF27 is co-opted in rhizobium symbiosis. BMC Plant Biol. 15: 260

*These authors contributed equally 


\section{Abstract}

\section{Background}

Strigolactones are a class of plant hormones of which biosynthesis is activated in response to phosphate starvation. This involves several enzymes, including the carotenoid cleavage dioxygenases 7 (CCD7) and CCD8 and the carotenoid isomerase DWARF27 (D27). D27 expression is known to be responsive to phosphate starvation. In Medicago truncatula and rice (Oryza sativa), this transcriptional response requires the GRAS-type proteins NSP1 and NSP2; both proteins are essential for rhizobium induced root nodule formation in legumes. In line with this, we questioned whether MtNSP1-MtNSP2 dependent MtD27 regulation is co-opted in rhizobium symbiosis.

\section{Results}

We provide evidence that MtD27 is involved in strigolactone biosynthesis in M. truncatula roots upon phosphate stress. Spatiotemporal expression studies revealed that this gene is also highly expressed in nodule primordia and the meristem and distal infection zone of mature nodules. A similar expression pattern was found for MtCCD7 and MtCCD8. Rhizobium lipo-chitooligosaccharide (LCO) application experiments revealed that, of these genes, MtD27 is most responsive in an MtNSP1- and MtNSP2-dependent manner. Symbiotic expression of $M t D 27$ requires components of the symbiosis signaling pathway, including MtDMI1, MtDMI2, MtDMI3/MtCCaMK and, in part, MtERN1. This in contrast to MtD27 expression upon phosphate starvation, which only requires MtNSP1 and MtNSP2.

\section{Conclusion}

Our data show that the phosphate starvation-responsive strigolactone biosynthesis gene MtD27 is also rapidly induced by rhizobium LCO signals in an MtNSP1- and MtNSP2dependent manner. Additionally, we show that MtD27 is co-expressed with MtCCD7 and $M t C C D 8$ in nodule primordia and in the infection zone of mature nodules. 


\section{BACKGROUND}

Legumes evolved the capacity to live in an intimate endosymbiosis with nitrogen-fixing rhizobium bacteria. To host rhizobia intracellularly, nodules are formed on the root of the plant. These nodules provide optimal physiological conditions to the bacteria to fix atmospheric nitrogen gas into ammonia. Recent studies have suggested a role for strigolactones in rhizobium symbiosis [1-6]. Here, we focus on expression of the strigolactone biosynthesis gene DWARF27 during the legume-rhizobium interaction.

Nodule formation is initiated upon perception of lipo-chitooligosaccharide (LCO) signals excreted by compatible rhizobium bacteria [7]. These signals mitotically activate root cortical and pericycle cells, resulting in the formation of a nodule primordium $[7,8]$. Rhizobium LCOs (also known as Nod factors) are also required to initiate an infection process to establish intracellular accommodation of the prokaryotic endosymbiont. This infection process starts in curled root hairs where a tube-like structure is formed intracellularly, which guides the rhizobia to the newly formed nodule primordium. There, the rhizobia are released as organelle-like structures (named symbiosomes), which remain surrounded by a plantderived membrane. These symbiosomes act as nitrogen-fixing units that provide ammonia to the plant cell in exchange of nutrients [7].

Rhizobium LCO signals are perceived by a specific set of LysM-type receptor kinases at the root epidermis. This activates a signaling cascade that is shared with a more ancient endosymbiosis: that between land plants and arbuscular mycorrhizal fungi [7]. This signaling cascade consists of a plasma membrane-localized LRR-type receptor kinase (named MtDMI2 in Medicago truncatula), a cation ion channel in the nuclear envelope (MtDMI1 in M. truncatula), and a nuclear-localized $\mathrm{Ca}^{2+} /$ calmodulin dependent protein kinase (MtCCaMK/ MtDMI3 in M. truncatula) [9-11]. Downstream of this cascade, CCaMK phosphorylates the transcriptional activator CYCLOPS, which orchestrates symbiotic root nodule development in conjunction with other transcription factors [12]. Among these are the GRAS-type transcriptional regulators NSP1 and NSP2 [13-15]. Experiments in heterologous systems have revealed that NSP2 can form a heterodimer with NSP1, suggesting a regulatory link between both proteins [16]. Both transcriptional regulators are essential for rhizobium symbiosis, but also promote mycorrhizal infection [17-20].

Under non-symbiotic conditions, NSP1 and NSP2 regulate the expression of DWARF27 (D27), which encodes a key enzyme in strigolactone biosynthesis [17,21]. Strigolactones are a class of plant hormones derived from all-trans- $\beta$-carotene [22-24]. They are produced 
mainly in the root of the plant and the biosynthesis of their basic structure involves at least three plastid-localized enzymes: the carotenoid isomerase D27 and the carotenoid cleavage dioxygenases 7 (CCD7) and CCD8 [22]. The subsequent activity of these three enzymes results in biosynthesis of carlactone, a precursor to the strigolactones. The conversion of carlactone to strigolactones is not completely resolved yet but there is strong evidence to support that this involves a cytochrome P450 enzyme, encoded by MAX1 in Arabidopsis (Arabidopsis thaliana) and rice (Oryza sativa) [25-28]. This biosynthetic pathway is under the control of a nutrient sensing mechanism and/or the nutrient status of the plant [29-31]. Especially in plants grown under phosphate-limited conditions, D27 expression and subsequent strigolactone production is markedly increased in an NSP1- and NSP2-dependent manner $[17,29]$.

Under phosphate limitation, strigolactones are exuded into the rhizosphere to attract arbuscular mycorrhizal fungi [31-34]. These obligatory biotrophic (symbiotic) fungi can sense strigolactones and respond with an increased hyphal branching, thereby, promoting colonization of plant roots $[32,35,36]$. The endomycorrhizal symbiosis facilitates nutrient exchange between fungus and plant. Mycelium that remains in the soil markedly increases the plant root capacity to access nutrients, especially immobile phosphates. The plant receives these nutrients from the fungi at the expense of carbohydrates [37]. Although strigolactones are not essential for establishment of an endomycorrhizal symbiosis, they contribute significantly to increasing root infection levels [33].

Several reports suggest also a role for strigolactones in legume nodule formation. In Medicago sativa and $M$. truncatula, application of $0.1 \mu \mathrm{M}$ GR24 was shown to promote nodule formation, whereas slightly higher concentrations inhibited nodule formation in $M$. truncatula [1,5]. In pea (Pisum sativum) and Lotus japonicus, strigolactone-deficient mutants were shown to produce less nodules, which could be rescued by external application of GR24 [2-4]. Furthermore, in M. truncatula root hairs, MtD27 and MtCCD8 expression is increased at 5 days post rhizobium inoculation [6]. This implies that MtD27 expression is regulated also in a symbiotic context. However, the precise regulatory network remains unknown.

Here, we show that in M. truncatula, MtD27 expression is induced by rhizobium LCOs in an MtNSP1- and MtNSP2-dependent manner, similar as found for the induction of MtD27 by phosphate starvation. However, only induction of MtD27 by rhizobium LCOs requires the symbiosis signaling cascade. Using promoter-reporter constructs, we show that MtD27 is expressed throughout nodule formation. After early activation in the epidermis, its expression becomes restricted to the nodule primordium and subsequently to the nodule 


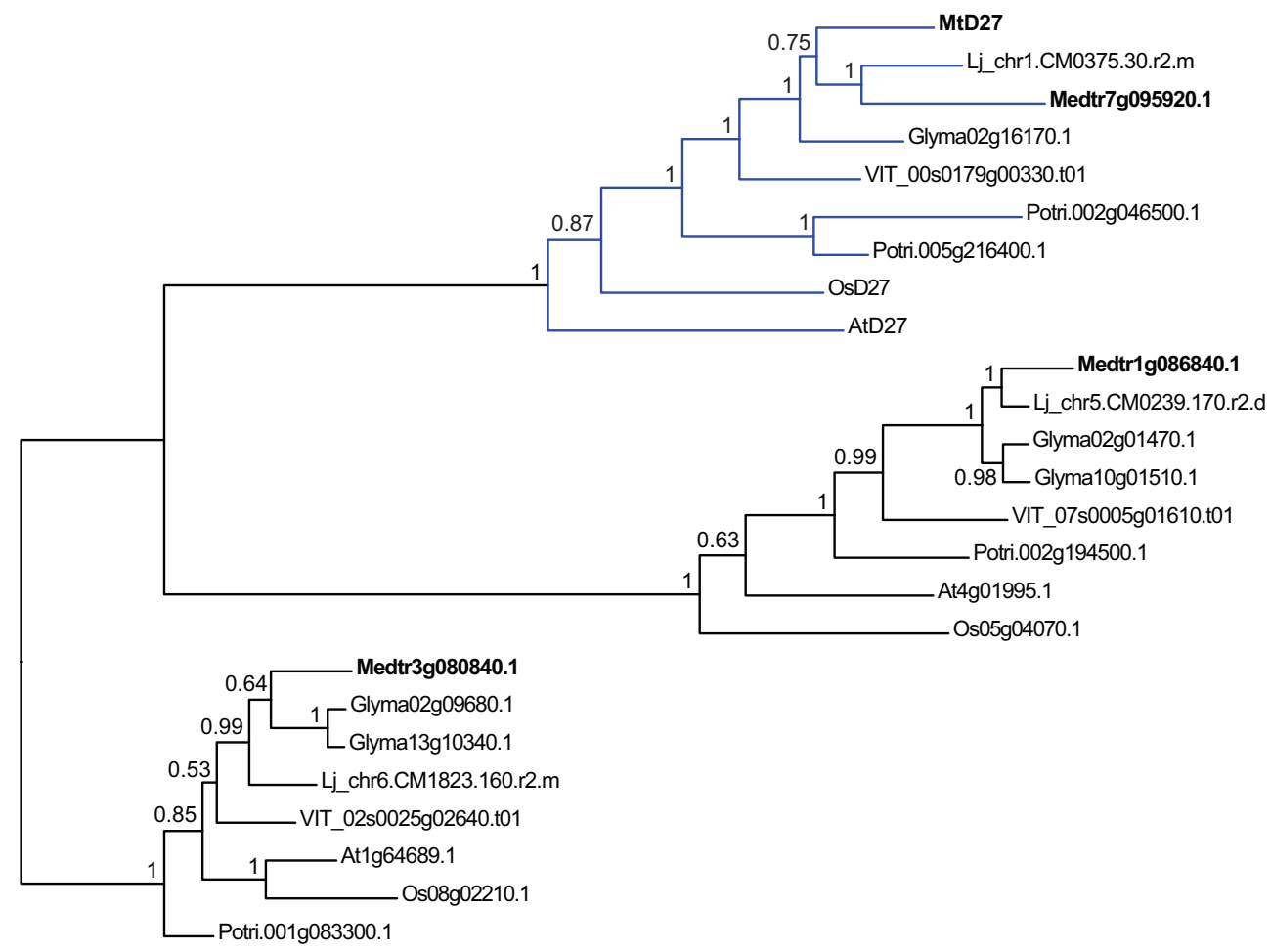

Figure 1. Bayesian phylogeny of D27 and D27-like proteins.

Phylogeny was reconstructed based on an alignment of D27 and D27-like proteins from Arabidopsis (At), soybean (Glycine max) (Glyma), Lotus japonicus (Lj), M. truncatula (Medtr), rice (Os), poplar (Populus trichocarpa) (Potri) and grapevine (Vitis vinifera) (VIT). Branch support is indicated by posterior probabilities. Terminals are labeled by their gene name or genbank identifier. Proteins identified from $M$. truncatula are highlighted in bold. The D27 orthology group containing rice OsD27 (gi|2549466546) and Arabidopsis AtD27 (At1G03055) is highlighted in blue. Mid-point rooting was applied for better tree visualization.

meristem and infection zone. Furthermore, we show that in nodule primordia and mature nodules MtD27 is co-expressed with MtCCD7 and MtCCD8.

\section{RESULTS}

\section{MtD27 Is Involved in Strigolactone Biosynthesis}

The M. truncatula D27 ortholog (Medtr1g471050) was identified previously [17]. By searching the $M$. truncatula genome annotation Mt4.0, we noted the presence of 3 close homologs of the original MtD27 gene. To get insight in the relation of these genes to MtD27, we conducted a phylogenetic analysis based on an alignment of D27(-like) proteins of 
A

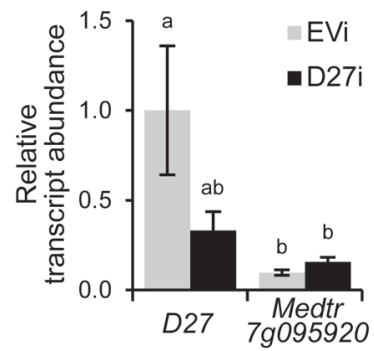

B

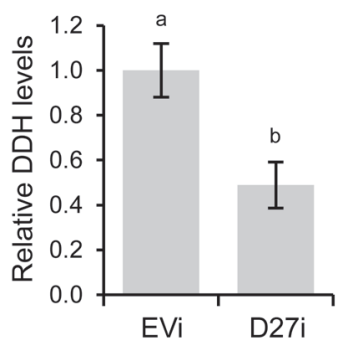

C

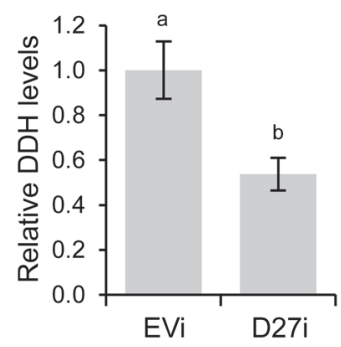

Figure 2. Effect of MtD27 knock-down on strigolactone biosynthesis.

(A) Relative transcript abundance as determined by qRT-PCR of MtD27 and Medtr7g095920 in M. truncatula transgenic roots expressing an empty vector control construct (EVi) or MtD27 RNAi construct (D27i). Relative transcript abundance was normalized against MtD27 transcript abundance in roots transformed with the empty vector control (EVi).

(B) Relative quantity of the strigolactone didehydro-orobanchol (DDH) (peak area/g FW) in root exudates collected from $M$. truncatula transgenic roots expressing an empty vector control construct (EVi) or MtD27 RNAi construct (D27i).

(C) Relative quantity of the strigolactone didehydro-orobanchol (DDH) (peak area/g FW) in root extracts collected from $M$. truncatula transgenic roots expressing an empty vector control construct (EVi) or MtD27 RNAi construct (D27i). Data shown represent the means of 4-5 biological replicates \pm SEM. Different letters above the bars indicate a statistical difference $(p<0.05$, Students' t-test).

different plant species, including rice and Arabidopsis (Figure 1). This showed that these sequences grouped in three separate phylogenetic clades (Figure 1), consistent with a previous report [38]. Interestingly, the D27 clade contains 2 proteins from M. truncatula: MtD27 [17] as well as a close homolog Medtr7g095920. This shows that $M$. truncatula contains 2 putative D27 genes that could function redundantly. Expression of MtD27 and subsequent strigolactone production is known to be induced by phosphate starvation [17]. To get first insight in whether Medtr7g095920 may also have a function in strigolactone production in M. truncatula roots, we examined its expression pattern. Analysis of publically available microarray data showed that Medtr7g095920 expression is relatively low and has limited overlap with expression of MtD27 [39]. In roots, Medtr7g095920 expression is not induced by phosphate starvation. This in contrast to MtD27 (Supplemental Figure 1) [17]. This suggests that under phosphate limiting conditions only MtD27 might be involved in strigolactone biosynthesis in $M$. truncatula roots.

Next, we determined whether MtD27 represents a functional enzyme in the strigolactone biosynthesis pathway. To this end, we generated $M$. truncatula compound plants bearing transgenic roots in which MtD27 expression was reduced through RNAi-mediated knockdown. This reduced MtD27 expression by $\sim 65 \%$, though with substantial variation (Figure 


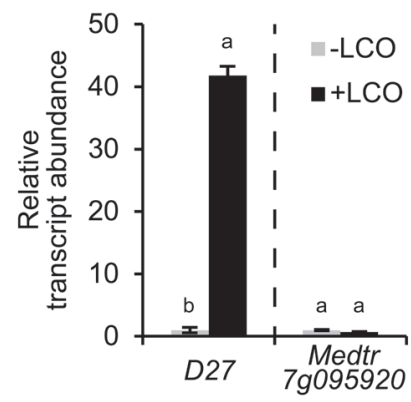

Figure 3. Relative transcript abundance of MtD27 and Medtr7g095920 after application of rhizobium LCOs.

Relative transcript abundance as determined by qRT-PCR of MtD27 and Medtr7g095920 in M. truncatula root susceptible zones 3 $\mathrm{h}$ after mock (-LCO) or S. meliloti LCO $\left(10^{-9} \mathrm{M}\right)(+\mathrm{LCO})$ treatment. Data shown represent the means of 2 biological replicates that each were analyzed in 3-fold (technical replicates) \pm SEM. For each gene, transcript abundance was normalized against that of the mock-treated wild type. Different letters above the bars indicate a statistical difference ( $p<0.05$, Students' t-test).

2A). In contrast, expression of Medtr7g095920 was not reduced, but lower than MtD27

(Figure 2A). Measurements of root extracts as well as root exudates collected from the MtD27 RNAi roots showed a 45-55\% reduction in strigolactone concentrations compared with that in the empty vector control (Figure 2B-C). This shows that MtD27 is involved in strigolactone biosynthesis and represents a functional ortholog of OsD27 from rice.

\section{Expression of MtD27 Is Increased upon Perception of Rhizobium LCOs in an MtNSP1- and MtNSP2-Dependent Manner}

To obtain insight in the symbiotic function of MtD27, we first determined whether its expression is responsive to rhizobium LCOs. Quantitative RT-PCR (qRT-PCR) reactions on RNA isolated from $M$. truncatula roots treated with Sinorhizobium meliloti LCOs $\left(\sim 10^{-9} \mathrm{M}\right)$ for $3 \mathrm{~h}$ revealed that expression of $M t D 27$ is strongly induced (Figure 3). To determine whether Medtr7g095920 is also responsive to rhizobium LCOs, we quantified its expression as well. This showed that, unlike MtD27, Medtr7g095920 is not responsive (Figure 3). The low expression of Medtr7g095920 in combination with its non-responsiveness to phosphate starvation and rhizobium LCOs, let us to decide to focus further studies on the symbiotic function of MtD27.

To acquire more insight in the symbiotic responsiveness of MtD27, we conducted a time series qRT-PCR experiment. To this end, $M$. truncatula seedlings were grown in Fåhraeus slides [40], a system optimized to study early responses induced by rhizobium LCOs in the root epidermis. $M$. truncatula roots of wild-type plants were treated with S. meliloti LCOs $\left(\sim 10^{-9} \mathrm{M}\right)$ for $0,1,2$ and $3 \mathrm{~h}$. Subsequently, total RNA was isolated from the so-called susceptible zone, a region of about $1 \mathrm{~cm}$ just above the root meristem. Expression analysis by qRT-PCR showed a slight induction of MtD27 already at $1 \mathrm{~h}$ post LCO application and a strong > 20-fold induction after 2-3 h compared with mock-treated roots (Figure 4A). This timing and induction level is comparable to that of MtENOD11, a gene frequently used as marker for rhizobium LCO-induced signaling in M. truncatula [16,41] (Figure 4A). 
A

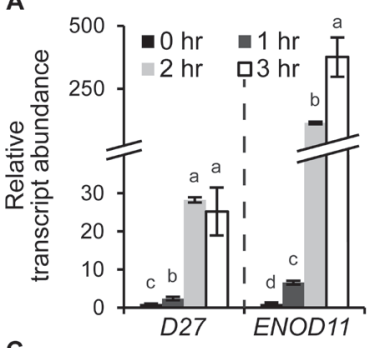

B

C
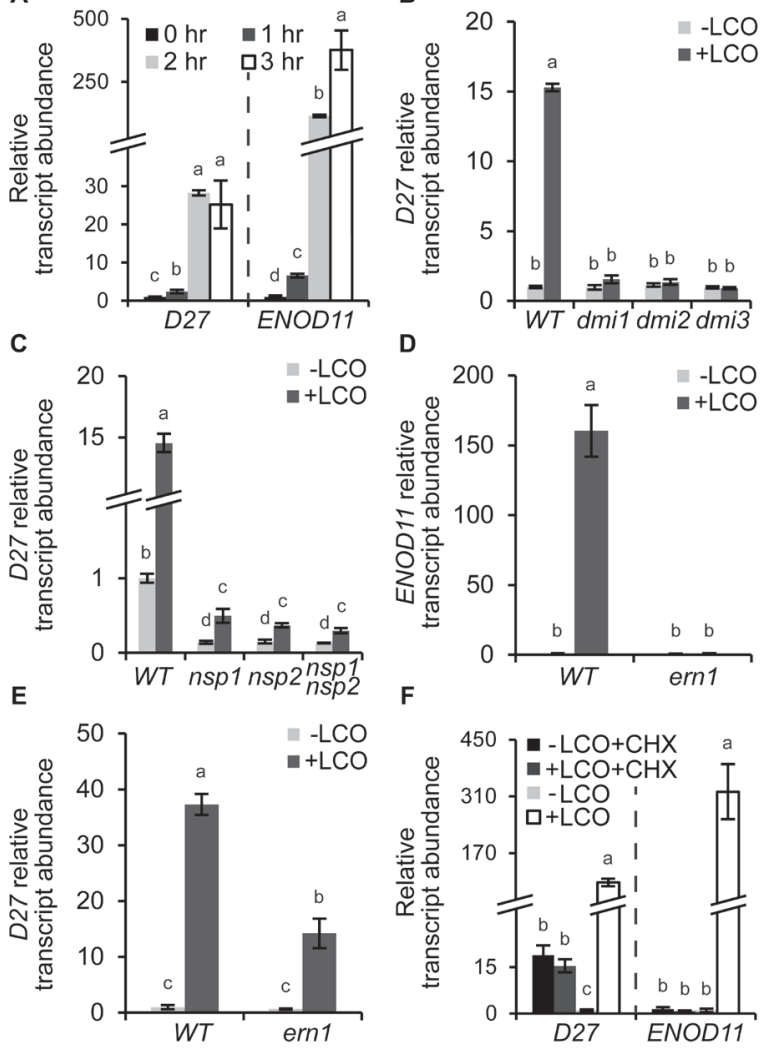

Figure 4. Symbiotic MtD27 expression is under direct control of S. meliloti LCO signaling.

(A) Relative transcript abundance as determined by qRT-PCR of MtD27 and MtENOD11 in M. truncatula root susceptible zones after $0,1,2$ or $3 \mathrm{~h}$ of $L C O$ treatment $\left(10^{-9} \mathrm{M}\right)$.

(B) Relative transcript abundance as determined by qRT-PCR of MtD27 at $3 \mathrm{~h}$ after mock (-LCO) or rhizobium LCO $\left(10^{-9} \mathrm{M}\right)(+\mathrm{LCO})$ treatment in wild type, Mtdmi1, Mtdmi2 and Mtdmi3.

(C) Relative transcript abundance as determined by qRT-PCR of MtD27 at $3 \mathrm{~h}$ after mock (-LCO) or rhizobium LCO $\left(10^{-9} \mathrm{M}\right)(+\mathrm{LCO})$ treatment in wild type, Mtnsp1, Mtnsp2 and Mtnsp1 Mtnsp2.

(D) Relative transcript abundance as determined by qRT-PCR of MtENOD11 at $3 \mathrm{~h}$ after mock (-LCO) or rhizobium LCO $\left(10^{-9} \mathrm{M}\right)(+\mathrm{LCO})$ treatment in wild type and Mtern1.

(E) Relative transcript abundance as determined by qRT-PCR of MtD27 at $3 \mathrm{~h}$ after mock (-LCO) or rhizobium LCO $\left(10^{-9} \mathrm{M}\right)(+\mathrm{LCO})$ treatment in wild type and Mtern1.

(F) Relative transcript abundance as determined by qRT-PCR of MtD27 and MtENOD11 after mock (-LCO) or rhizobium $\mathrm{LCO}\left(10^{-9} \mathrm{M}\right)(+\mathrm{LCO})$ treatment, in the presence or absence of $50 \mu \mathrm{M}$ cycloheximide (CHX).

Data shown represent the means of 3 biological replicates that each were analyzed in 3-fold (technical replicates) \pm SEM. For each gene, transcript abundance was normalized against that of the mocktreated wild type. Different letters above the bars indicate a statistical difference $(p<0.05$, Students' t-test). 
Induction of MtENOD11 expression by rhizobium LCOs requires a signaling module downstream of LCO perception consisting of MtDMI1, MtDMI2 and MtDMI3/MtCCaMK (the common symbiosis signaling module) and the transcriptional regulators MtNSP1, MtNSP2 and MtERN1 [42-44]. To investigate whether induction of MtD27 expression by rhizobium LCOs is also dependent on this signaling module, we conducted qRT-PCR on LCO-susceptible root zones of these mutant plants, using MtENOD11 as control. This revealed that MtD27 expression is not induced in Mtdmi1, Mtdmi2 and Mtdmi3 mutant roots (Figure 4B), indicating that symbiotic induction of $M t D 27$ requires the common signaling module genes. Previously, we demonstrated that MtD27 expression in non-inoculated roots is dependent on MtNSP1 and MtNSP2 [17]. Here, we found that MtD27 expression is still induced by LCOs in an Mtnsp1, Mtnsp2 and Mtnsp1Mtnsp2 mutant background, albeit significantly lower than in wild-type roots ( $\sim 2-3$-fold vs $\sim 15$-fold, respectively) (Figure $4 \mathrm{C}$ ). Next, we monitored MtD27 expression in the Mtern1 mutant, in which LCO-induced MtENOD11 expression is blocked [45] (Figure 4D). In this mutant, the induction of MtD27 by rhizobium LCOs is about half of that in the wild type (Figure 4E). These results show that MtD27 is a rhizobium LCO-responsive gene whose expression in a symbiotic context is largely dependent on the common signaling module and the transcriptional regulators MtNSP1, MtNSP2 and, in part, MtERN1.

Previously, it was shown in Vicia sativa that the LCO inducibility of early nodulin genes is indirect, as an inhibition of protein synthesis by cycloheximide ( $\mathrm{CHX}$ ) blocks early nodulin genes expression [46]. As MtD27 displays a comparable expression pattern as MtENOD11, we tested whether this gene is a primary target of rhizobium LCO signaling. To this end, M. truncatula seedlings were grown in Fåhraeus slides for 3 days and pre-treated with 50 $\mu \mathrm{M}$ CHX for 30 minutes prior to LCO treatment $\left(10^{-9} \mathrm{M}, 3 \mathrm{~h}\right)$. The expression of MtD27 and MtENOD11 in the susceptible root zone was monitored by qRT-PCR. This showed that in control plants, MtD27 expression is elevated by $\mathrm{CHX}$ treatment (Figure $4 \mathrm{~F}$ ), suggesting that active protein synthesis is required to keep MtD27 expression at basal levels. In addition, it showed that, like MtENOD11, MtD27 expression is induced by rhizobium LCOs in the absence of $\mathrm{CHX}$, but not in the presence of $\mathrm{CHX}$ (Figure 4F). This indicates that induction of $M t D 27$ expression by rhizobium LCOs requires new protein synthesis and therefore suggests that induction of MtD27 by rhizobium LCOs is indirect.

\section{MtD27 Is Co-Expressed with MtCCD7 and MtCCD8 During Nodule Formation}

To obtain insight in the symbiotic function of $M t D 27$, its spatiotemporal expression pattern is analyzed. For this, a 1 kb fragment representing the 5' region upstream of the translational start site was cloned into a binary transformation vector in front of a $\beta$-glucuronidase (GUS)- 

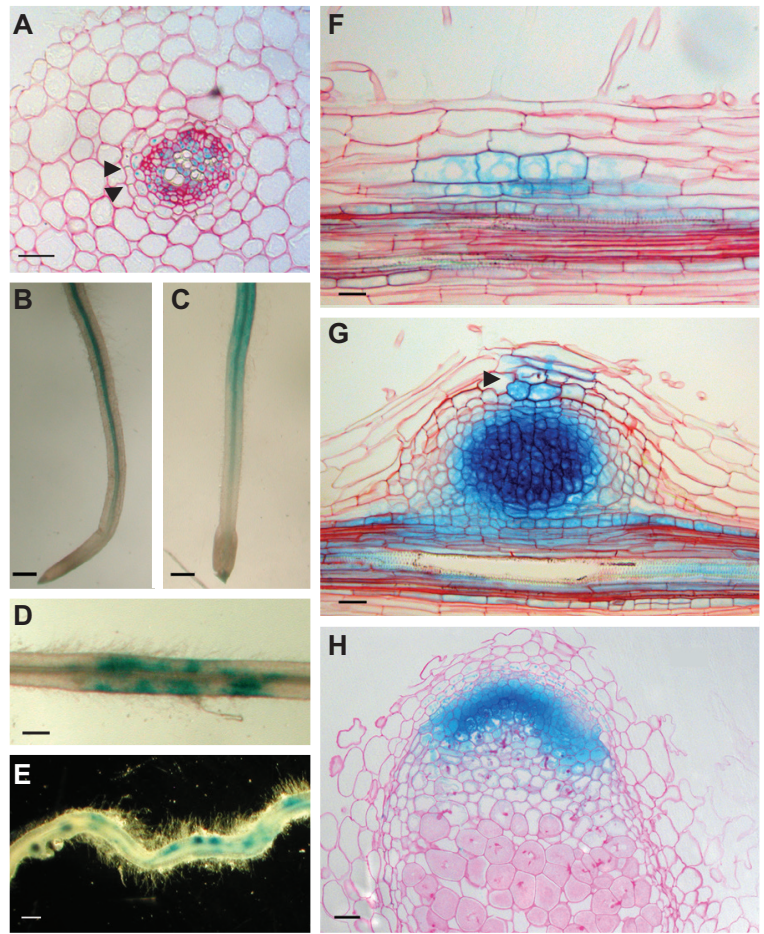

Figure 5. Spatial expression pattern of MtD27 in M. truncatula roots and nodules.

(A) Cross-section through a non-inoculated root. Arrowheads indicate casparian strips, which mark the endodermal cell layer.

(B) Mock-treated control root.

(C) Root treated with S. meliloti LCOs $\left(10^{-9} \mathrm{M}\right)$ for $3 \mathrm{~h}$.

(D) Root at four days post inoculation (dpi) with S. meliloti strain 2011.

(E) Root at $7 \mathrm{dpi}$.

(F) Longitudinal section through a root at $2 \mathrm{dpi}$.

(G) Longitudinal section through a nodule primordium (7 dpi). The infection thread is indicated with an arrowhead.

(H) Longitudinal section through an eighteen-day-old nodule.

The MtD27 spatial expression pattern was analyzed in $M$. truncatula transgenic roots expressing an MtD27 promoter-reporter GUS construct. Scale bars are equal to $25 \mu \mathrm{m}$ (A, F and G), $0.5 \mathrm{~mm}$ (B-E) and $50 \mu \mathrm{m}(\mathrm{H})$. Sections were counterstained with Ruthenium Red.

encoding sequence. This construct was used to create $M$. truncatula compound plants carrying transgenic roots. In non-inoculated plants, grown on buffered nodulation medium (BNM) containing no nitrate but a relatively high phosphate concentration $\left(0.5 \mathrm{mM} \mathrm{PO}_{4}{ }^{3-}\right)$, MtD27 expression was observed in the vasculature and pericycle (Figure 5A-B). LCO ( 10-9 $\mathrm{M})$ treatment for $3 \mathrm{~h}$ induced MtD27 expression in the root epidermis (Figure 5C). 
Next, we determined the spatial expression of MtD27 following inoculation with S. meliloti strain 2011. This showed that at 4 days post inoculation (dpi), the pMtD27::GUS transgenic roots showed a patched GUS staining, which was associated with rhizobium root hair infections (Figure 5D). At $7 \mathrm{dpi}$, the expression in the root ceased but GUS activity accumulated in nodule primordia (Figure $5 \mathrm{E}$ ). Sectioning nodule primordia revealed expression of pMtD27::GUS in dividing pericycle and cortical cells (Figure 5F). This expression maintains in the developing nodule primordium (Figure 5G) but becomes more restricted in the mature nodule, where it is visible only in the meristem and distal infection zone (Figure $\mathbf{5 H}$ ). Taken together, the LCO responsiveness and its spatial expression pattern in nodule primordia, nodule meristem and infection zone strongly support a symbiotic function of MtD27.

As strigolactone biosynthesis requires at least three enzymes, we tested whether besides MtD27, also CCD7 and CCD8 are responsive to rhizobium LCOs in M. truncatula. To this end, we first identified the putative orthologs of $C C D 7$ and $C C D 8$ from the M. truncatula genome based on homology to Arabidopsis genes. This revealed that one copy of CCD7 (Medt7g045370; MtCCD7) and two copies of CCD8 (Medtr3g109610 and Medtr7g063800) are encoded in the $M$. truncatula genome (Supplemental Figure 2). Medtr3g109610 was described previously as MtCCD8 [6]. Analysis of the Medicago gene atlas showed that MtCCD8 and Medtr7g063800 show a similar expression pattern, though expression of MtCCD8 is about 10- to 20-fold higher when compared to Medtr7g063800 [39]. This also applies to expression of both genes during phosphate starvation and rhizobium LCO application (Supplemental Figure 3) and therefore we decided to focus on MtCCD 8 for the remainder of this study. Expression analysis by qRT-PCR on samples taken from plants grown in Fåhraeus slides showed that MtCCD7 and MtCCD 8 were not induced at $3 \mathrm{~h}$ post rhizobium LCO application (Figure 6A). In contrast, in roots grown on agar-solidified Fåhraeus medium supplemented with amino ethoxyvinylglycine (AVG), expression of MtCCD8 is induced 5-fold 3-h after application of rhizobium LCOs, whereas MtCCD7 expression was not substantially affected (Figure 6B).

To determine the spatial-temporal expression pattern of MtCCD7 and MtCCD8, promoterreporter GUS constructs were created and introduced in $M$. truncatula roots using $A$. rhizogenes-mediated transformation. We noted a basal expression pattern of both genes in the young root tip, including the susceptible zone and did not observe a discernible change in expression pattern of neither MtCCD7 nor MtCCD8 following LCO treatment (Supplemental Figure 4). This suggests that of the MtD27-MtCCD7-MtCCD8 biosynthesis module, MtD27 expression is most strictly controlled in a spatial-temporal manner. However, 

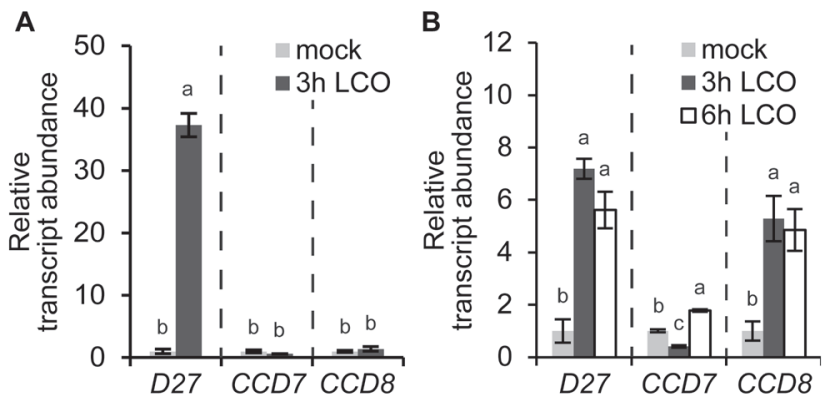

Figure 6. Expression of MtD27, MtCCD7 and MtCCD8 upon treatment with rhizobium LCOs.

(A) Relative transcript abundance as determined by qRT-PCR of MtD27, MtCCD7 and MtCCD8 in $M$. truncatula root susceptible zones $3 \mathrm{~h}$ after mock or rhizobium LCO $\left(10^{-9} \mathrm{M}\right)$ treatment. RNA was isolated from plants grown in Fåhraeus slides.

(B) Relative transcript abundance as determined by qRT-PCR of MtD27, MtCCD7 and MtCCD8 in M. truncatula root susceptible zones 3 or $6 \mathrm{~h}$ after mock or rhizobium LCO $\left(10^{-9} \mathrm{M}\right)$ treatment. RNA was isolated from plants grown on agar-solidified Fåhraeus medium supplemented with $1 \mu \mathrm{M}$ AVG.

Data shown represent the means of 2-3 biological replicates that each were analyzed in 3-fold (technical replicates) \pm SEM. For each gene, transcript abundance was normalized against that of the mock-treated wild type. Different letters above the bars indicate a statistical difference $(p<0.05$, Students' t-test).

upon inoculation with S. meliloti, it showed that in young (two-day-old) nodule primordia, MtCCD7 and MtCCD8 are co-expressed with MtD27 (Figure 5F and 7A-B). Moreover, in mature nodules, MtD27, MtCCD7 and MtCCD8 are co-expressed in the nodule meristem and distal infection zone (Figure $5 \mathbf{H}$ and 7 C-D).

Previous studies on strigolactone deficient mutants revealed that strigolactones, at least in part, contribute to root nodule formation and functioning [2-4]. To test whether MtD27 function is required for rhizobium symbiosis, we determined the nodulation phenotype of MtD27 RNAi roots. Under these conditions, the MtD27 RNAi construct reduced MtD27 expression by $>90 \%$ (Supplemental Figure 5A). Examination of the MtD27 RNAi roots showed that they can be effectively nodulated (Supplemental Figure 5B). Sectioning of $\sim 40$ nodules did not reveal any discernible difference in nodule morphology between nodules formed on control or MtD27 RNAi roots (Supplemental Figure 5C-D). This suggests that either the reduction in MtD27 expression is not sufficient to cause a phenotype or that MtD27 is not essential for root nodule development and functioning. 

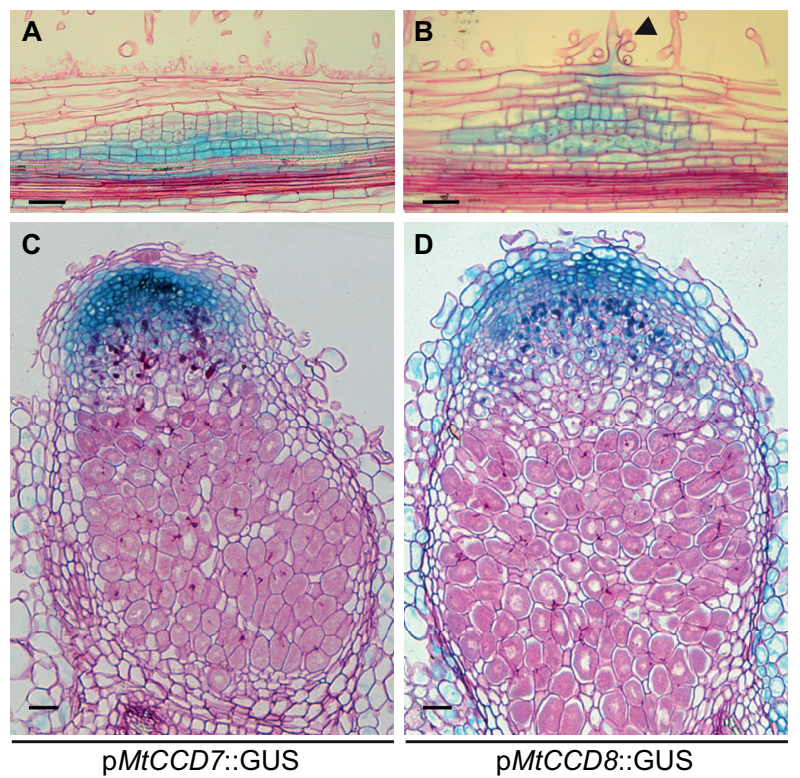

Figure 7. Spatial expression pattern of MtCCD7 and MtCCD8 in M. truncatula nodule primordia and mature nodules.

(A) Longitudinal section through a root expressing the pMtCCD7::GUS construct 2 days post inoculation (dpi) with S. meliloti strain 2011.

(B) Longitudinal section through a root expressing the pMtCCD8::GUS construct at $2 \mathrm{dpi}$. Arrowhead points at an infection thread growing inside the root hair cell.

(C) Longitudinal section through a mature nodule expressing the pMtCCD7::GUS construct.

(D) Longitudinal section through a mature nodule expressing the pMtCCD8::GUS construct.

Expression patterns were analyzed in $M$. truncatula transgenic roots expressing promoter-reporter GUS constructs. Scale bars are equal to $50 \mu \mathrm{m}$. Sections were counterstained with Ruthenium Red.

The Induction of MtD27 Expression by Phosphate Deprivation Is Independent of the Common Symbiotic Signaling Cascade

Besides rhizobium LCOs, also phosphate starvation elevates MtD27 expression in an NSP1- and NSP2-dependent manner [17]. Induction of MtD27 by rhizobium LCOs requires MtDMI3/MtCCaMK and, in part, MtERN1. DMI3/CCaMK is positioned directly upstream of NSP1-NSP2 in the LCO signaling pathway $[7,47]$, whereas ERN1 functions in concert with NSP1-NSP2 to regulate expression of MtENOD11 [48]. In line with this, we questioned whether MtDMI3/MtCCaMK and MtERN1 are also required to induce MtD27 expression in response to a low phosphate status. First, we determined to what extent the spatial MtD27 expression in $M$. truncatula roots is affected by different phosphate regimes. To this end, transgenic $M$. truncatula plants carrying the MtD27 promoter-GUS reporter construct were grown in perlite for 2 weeks at high phosphate $\left(200 \mu \mathrm{M} \mathrm{PO}_{4}{ }^{3-}\right)$-containing medium and 

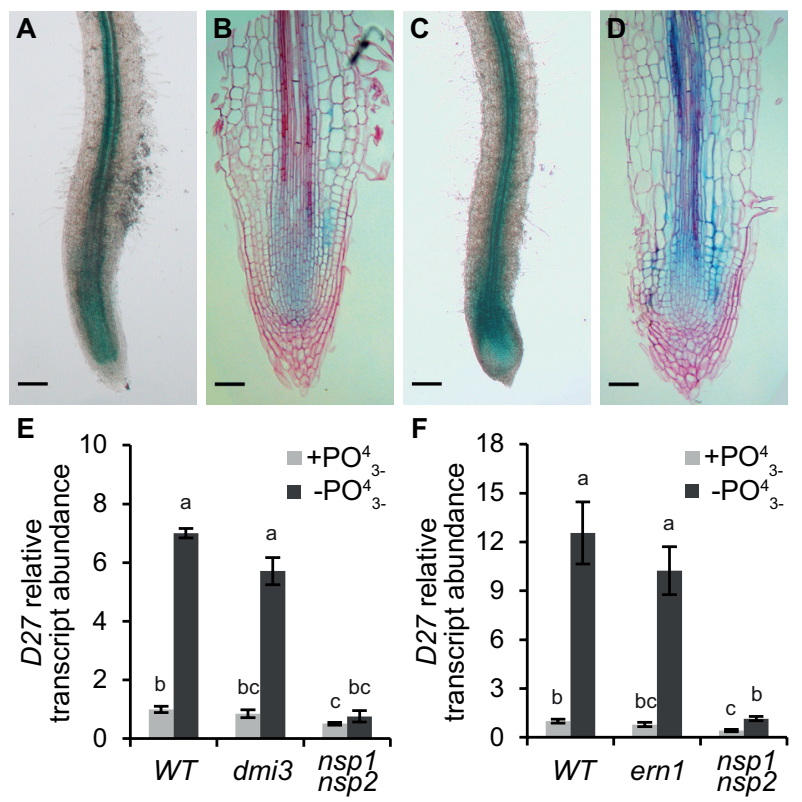

Figure 8. Spatial expression pattern of MtD27 upon phosphate starvation.

(A) Transgenic M. truncatula root expressing $p M t D 27:: G U S$ grown in full nutrient condition (200 $\mu \mathrm{M}$ $\left.\mathrm{PO}_{4}{ }^{3-}\right)$.

(B) Longitudinal section of the root shown in (A).

(C) Expression of $p M t D 27:: G U S$ in $M$. truncatula transgenic roots after 5 days of phosphate starvation $\left(0 \mu \mathrm{M} \mathrm{PO}_{4}{ }^{3-}\right)$.

(D) Longitudinal section of the root shown in (C).

(E) Relative transcript abundance as determined by qRT-PCR of MtD27 in wild type (WT) and the Mtdmi3 mutant and Mtnsp1 Mtnsp2 double mutant after 2 days of phosphate starvation ( $\left.0 \mu \mathrm{M} \mathrm{PO}_{4}{ }^{3-}\right)$.

(F) Relative transcript abundance as determined by qRT-PCR of MtD27 in WT and the Mtern1 mutant and Mtnsp1 Mtnsp2 double mutant after 2 days of phosphate starvation.

Scale bars are equal to $250 \mu \mathrm{m}$ in (A) and (C) and $50 \mu \mathrm{m}$ in (B) and (D). Sections were counterstained with Ruthenium Red. Data in (E-F) represent the means of 3 biological replicates that each were analyzed in 3-fold (technical replicates) \pm SEM. For each gene, transcript abundance was normalized against that of the mock-treated wild type. Different letters above the bars indicate a statistical difference $(p<0.05$, Students' t-test).

subsequently transferred to no phosphate $\left(0 \mu \mathrm{M} \mathrm{PO}_{4}{ }^{3-}\right)$ medium. Plant roots were stained histochemically for GUS activity 5 days after the transfer and compared to control roots. In all plants, GUS staining could be observed in the stele of the root as well as in the root apical meristem (Figure 8A-B). Phosphate-starved roots displayed a much more intense staining than control roots, which was most clear in the root apical meristem (Figure $8 \mathrm{C}-\mathrm{D}$ ). However, unlike treatment with rhizobium LCOs, phosphate starvation did not change the spatial expression pattern of MtD27. 
Next, we determined whether MtDMI3/MtCCaMK and MtERN1 are also essential to mediate MtD27 expression by phosphate starvation. To this end, $M$. truncatula Mtdmi3 and Mtern1 knockout mutants were grown in an aeroponic system containing medium with high phosphate ( $\left.200 \mu \mathrm{M} \mathrm{PO}_{4}{ }^{3-}\right)$ and subsequently transferred to a medium containing no phosphate for 2 days. To determine the expression of MtD27, qRT-PCR was conducted on RNA isolated from the bottom 2-3 cm of the root. This study reveals that in both mutants induction of MtD27 in response to phosphate deprivation is similar to the induction found in roots of wild-type plants (Figure $\mathbf{8 E - F}$ ). This indicates that the phosphate response of MtD27 expression is independent of the symbiotic signaling genes MtDMI3/MtCCaMK and MtERN1.

\section{Discussion}

Several lines of evidence indicate that strigolactones play a role in the legume rhizobium symbiosis [1-6]. Here, we showed that expression of MtD27, a gene that is acting in the strigolactone biosynthesis pathway, is strongly elevated by rhizobium LCO-induced signaling. Additionally, we found that this gene is co-expressed with MtCCD7 and MtCCD8 in nodule primordia as well as in the infection zone of mature nodules. This suggests a putative function for these strigolactone biosynthesis genes during several stages of the legumerhizobium interaction.

Studies with the strigolactone analog GR24 revealed a nodulation-enhancing effect when applied exogenously $[1,5]$. In line with this, a severe reduction in endogenous strigolactone levels due to mutations in $c c d 7$ or $c c d 8$ is linked to a moderate decrease in nodulation efficiency [2-4]. Foo and Davies [2] conclude that, although strigolactones influence nodule initiation, they are not essential. We were unable to confirm these results in MtD27 RNAi-knockdown roots of $M$. truncatula. We cannot rule out that in our MtD27 RNAi experiments, MtD27 expression is not sufficiently reduced to cause such moderate nodulation phenotype. Alternatively, phenotypes caused by an altered D27 function may be weaker to that of phenotypes of $c c d 7$ or $c c d 8$ mutants. This hypothesis finds support by studies in Arabidopsis and rice, where shoot branching phenotypes were much more severe in $c c d 7$ and $c c d 8$ mutants when compared to $d 27$ [21,38]. Based on this, Waters et al. [38] speculate on residual bioactive compounds present in Atd27 mutants. If such a residual bioactive compound also exists in $M$. truncatula, it is possible that its activity is sufficient for proper nodule initiation and development. 


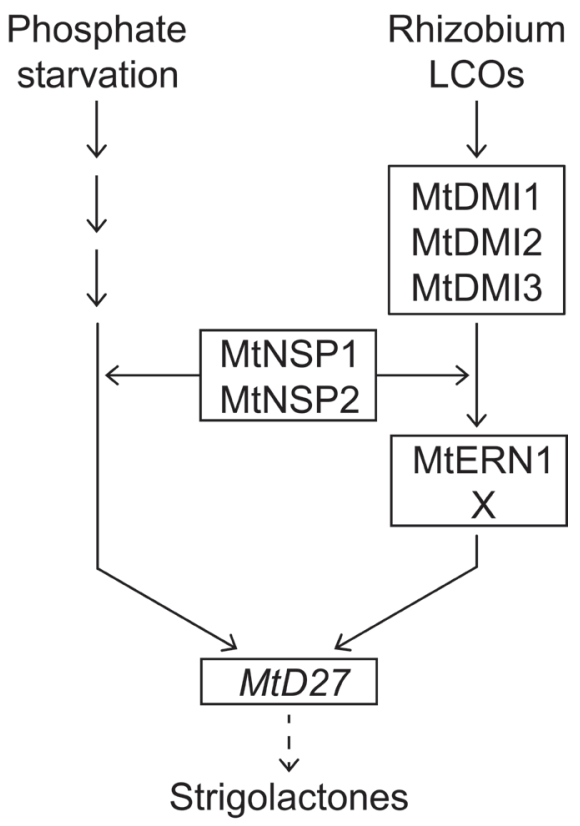

Figure 9. Schematic model depicting the induction of MtD27 expression by phosphate starvation and rhizobium LCO-induced signaling.

Rhizobium LCOs activate expression of MtD27 through the common symbiosis signaling module, consisting of MtDMI1, MtDMI2 and MtDMI3. Downstream of this module MtERN1 is required for full induction of MtD27. MtERN1 function is partly redundant, suggesting that MtERN1 might function in conjunction with or redundant to another unknown transcriptional regulator, indicated as $X$. This unknown factor might be MtERN2, a transcriptional regulator closely related to MtERN1 [48]. The pathway leading to activation of MtD27 expression by phosphate starvation remains unknown. The GRAS proteins MtNSP1 and MtNSP2 are required for expression of MtD27 during phosphate starvation and following rhizobium LCO application. We propose that these proteins function in parallel to both signaling pathways, as previously already suggested for the LCO signaling cascade $[7,15]$.

It remains currently unknown how strigolactones promote nodule initiation. One possible mechanism is through promoting the formation of a nodular auxin maximum. Mathematical modelling predicts that such maximum is most likely created through a local reduction in the auxin transport capacity in the root cortex [49]. Such reductions in root auxin transport capacity have been observed following rhizobial inoculation [50,51]. Strigolactone-deficient mutants of Arabidopsis show elevated auxin transport in both shoots and roots [52]. It is proposed that strigolactones act by targeting the PIN auxin-efflux carriers at both the gene expression and protein level [53-57]. Therefore, it is possible that rhizobium-induced strigolactone biosynthesis will affect auxin transport and as such contributes to create and/or maintain an auxin maximum during nodule formation. However, it is unlikely that 
strigolactones alone are sufficient to reduce the auxin transport capacity upon perception of rhizobium LCOs, as strigolactone-deficient mutants still form nodules, although less than wild-type plants $[2,3]$. Possibly, strigolactones function redundantly to another signal, like for example cytokinin [51,58-60] or flavonoids [61].

MtD27 expression is elevated within 1-2 $\mathrm{h}$ post LCO application, by which it is among the earliest responsive genes. This transcriptional activation is under control of the rhizobium LCO signaling network, which includes MtDMI2, MtDMI1, MtDMI3/MtCCaMK, MtNSP1, MtNSP2 and, in part, MtERN1. We found that also expression of MtCCD7 and MtCCD8 was induced following application of rhizobium LCOs. However, these responses were less pronounced when compared to MtD27. Expression of MtCCD7 was only slightly affected by application of rhizobium LCOs, whereas induction of MtCCD 8 was dependent on the growth system. Spatial-temporal expression analysis revealed that, in a symbiotic context, especially MtD27 expression is strictly controlled in a spatial-temporal manner. Under nonsymbiotic conditions, MtD27 is mainly expressed in the stele of the root, whereas rhizobium LCOs activate expression in the root epidermis. Such clear spatial-temporal regulation was not observed for MtCCD7 nor MtCCD8, as both genes have a much broader expression pattern under non-symbiotic conditions. Why MtD27 is strictly controlled under symbiotic conditions remains unknown. Expression of MtCCD7 and MtCCD 8 might not be rate limiting, or alternatively, induction of MtD27 by rhizobium LCOs might be part of a priming response that prepares epidermal cells for the infection process [6].

Our data hint at a putative role for strigolactones in mature nodules as MtD27, MtCCD7 and MtCCD 8 are co-expressed in the nodule meristem and distal infection zone. This may suggest that strigolactones promote meristem functioning and/or rhizobial infection. Recently, it was shown that MtD27 and MtCCD8 are transcriptionally induced in infected root hairs [6]. Our data also indicate expression of MtD27 and MtCCD8 in cells that contain growing infection threads in the root nodule, supporting a putative function for MtD27 and $M t C C D 8$ in the infection process. Furthermore, induction of $M t D 27$ expression by rhizobium LCOs is partly dependent on MtERN1, a transcription factor required for infection thread development [45].

MtD27 is also transcriptionally activated by phosphate starvation stress and this induction is dependent on MtNSP1 and MtNSP2 [17]. We studied the spatial regulation of MtD27 in response to phosphate starvation and found that the spatial expression pattern remains unchanged but MtD27 expression is increased in the stele and apical root meristem. The transcriptional activation of $M t D 27$ in response to the phosphate status in the environment 
coincides with an increased exudation of strigolactones [29,30,34]. Generally, it is anticipated that this response is contributing to the attraction of endomycorrhizal fungi, which enhance phosphate acquisition from the environment $[33,36]$. We tested whether the induction of MtD27 by phosphate starvation is dependent on the common signaling pathway as well as whether this response is (partially) dependent on MtERN1. Neither signaling components were involved in the induction of MtD27 expression by phosphate starvation. This indicates that the signaling pathways regulating transcriptional activation of MtD27 by rhizobium LCOs and phosphate starvation only share NSP1 and NSP2 (Figure 9). Interestingly, MtD27 transcript abundance is reduced in an Mtnsp1Mtnsp2 mutant background when compared to wild type [17]. Furthermore, we found that induction of this gene upon phosphate stress or LCO signaling still occurs in these mutants, although at very moderate levels (Figure 4E and 8 F). Taken together, this supports the hypothesis that MtNSP1 and MtNSP2 function in a parallel pathway to facilitate induction of MtD27 by rhizobium LCOs and phosphate starvation stress, rather than being part of the primary signaling cascades $[7,15]$ (Figure 9).

The D27-CCD7-CCD8 biosynthetic module is largely conserved in higher plants. As we showed that MtD27 is transcriptionally activated in a spatial-temporal manner in response to rhizobium LCOs in $M$. truncatula roots, this gene may represent an excellent marker gene to study rhizobium-induced signaling in a phylogenetic context.

\section{Conclusions}

Here, we showed that in M. truncatula, MtD27 expression is rapidly increased upon perception of rhizobium LCOs. The gene remains expressed in the dividing cells of the nodule primordium and at subsequent stages its expression becomes confined to the nodule meristem and distal infection zone of the mature nodule. Analysis of the expression of MtCCD7 and MtCCD8 showed that they are co-expressed with MtD27 in nodule primordia and mature nodules. Additionally, we show that symbiotic expression of MtD27 as well as its expression during phosphate starvation is dependent on the GRAS-type regulators MtNSP1 and MtNSP2. This suggests that the NSP1-NSP2-D27 regulatory unit is co-opted to function in rhizobium symbiosis. 


\section{Methods}

\section{Plant Materials and Growth Conditions}

M. truncatula Jemalong A17, dmi1-1 (C71) [9,42], dmi2-1 (TR25) [62], dmi3-1 (TRV25) [10], nsp1-1 (B85) [14,42], nsp2-2 (0-4) [13,43], nsp1 nsp2 [17] and ern1 (bit1-1) [45] were used in this study. Plants were grown in a growth chamber at $20^{\circ} \mathrm{C}$ under $16 / 8$ photoperiod at $50 \mu$ moles of photons $\mathrm{m}^{-2} \mathrm{~s}^{-1}$. For gene expression studies, plants were grown in modified Fåhraeus slides [40]. A single germinated seedling was placed in each slide and medium was refreshed every $24 \mathrm{~h}$. Seedlings were grown for 3 days, before subjecting them to a $3 \mathrm{~h}$ treatment with Sinorhizobium meliloti LCOs $\left(\sim 10^{-9} \mathrm{M}\right)$, unless stated otherwise. Subsequently, $1-\mathrm{cm}$ root segments were cut just above the root tip and snap-frozen in liquid nitrogen.

For analysis of transcriptional induction of $M t D 27$ by phosphate starvation, plants were grown as previously described [17]. This time, plants were grown for 2.5 weeks before subjecting them to a two-day phosphate starvation regime. Subsequently, 2 to $3-\mathrm{cm}$ root segments including the root tip were cut and snap-frozen in liquid nitrogen.

\section{Phylogenetic Reconstruction}

Predicted proteomes of Glycine max (Wm82.a2.v1) [63], Lotus japonicus (Lj2.5) [64], Medicago truncatula (Mt4.0v1) [65], Oryza sativa (v7.0) [66], Populus trichocarpa (v3.0) [67] and Vitis vinifera (Genoscope.12X) [68] were obtained through Phytozome 10 (http:// phytozome.jgi.doe.gov/). These proteomes were searched by BLAST using $A$. thaliana proteins (TAIR10, www.arabidopsis.org) as query. For phylogenetic reconstruction, full length (predicted) protein sequences were aligned using MAFFT v7.017 [69] implemented in Geneious R6 (Biomatters, Auckland, New Zealand), using default parameter settings. After manual inspection, alignments were used for tree building using MrBayes 3.2.2 [70] implemented in Geneious R6, using default parameter settings, with the exception of the rate matrix, for which wag was used. Midpoint rooting was performed for better tree visualization.

\section{Vectors and Constructs}

For promoter-GUS reporter assays, a $\sim 1 \mathrm{~kb}(M t D 27)$ or $\sim 2 \mathrm{~kb}$ (MtCCD7 and MtCCD8) fragment upstream of the translational start site was amplified from $M$. truncatula Jemalong A17 genomic DNA using the primers listed in Supplemental Table 1. The pMtD27 fragment and a $\beta$-glucuronidase (GUS)-encoding sequence were recombined into a pDONR-L4L1 and pDONR-L1L2, thereby creating pENTR4-1_pMtD27 and pENRT1-2_GUS, respectively. These two constructs were combined with a pENTR2-3_t35S and subsequently recombined into 
the binary destination vector pKGW-RR-MGW by a multisite gateway reaction (Invitrogen, Carlsbad, USA). The putative promoter fragments of MtCCD7 and MtCCD 8 were recombined into a pENTR-D-TOPO vector (Invitrogen, Carlsbad, USA), creating pENTR1-2_pMtCCD7 and pENTR1-2_pMtCCD8, respectively. Subsequently, both constructs were recombined into pKGWFS7-RR, containing a GUS-GFP fusion reporter, by a single-site gateway reaction (Invitrogen, Carlsbad, USA), creating pKGWFS7-RR_pMtCCD7-GUS and pKGWFS7-RR_ pMtCCD8-GUS, respectively.

For RNAi-mediated knockdown of MtD27, a 268-bp fragment was amplified from $M$. truncatula Jemalong A17 root cDNA, using primer pairs MtD27i-F and MtD27i-R (see Supplemental Table 1), and cloned into pENTR-D-TOPO (Invitrogen, Carlsbad, USA). The MtD27 RNAi fragment was recombined into the DsRed-modified gateway vector pK7GWIWG2(II)-RR driven by the CaMV35S promoter [71] to obtain the binary construct pK7GWIWG2(II)-RR-p35S-MtD27-RNAi. For the empty vector control, a pENTR containing a 70 bp multiple cloning site was recombined into pK7GWIWG2(II)-RR to obtain the binary plasmid pK7GWIWG2(II)-RR-p35S-RNAi-control. All vectors used in this study contain pAtUBQ10::DsRED1 as selection marker [72]. All cloning vectors are available upon request from Plant Systems Biology (V.I.B.-Ghent University).

\section{Plant Transformation and Treatments}

A. rhizogenes-mediated root transformation of $M$. truncatula was performed as previously described [72]. For treatments with rhizobium LCOs, compound plants were transferred to agar-solidified buffered nodulation medium (BNM, 0.9\% Daishin agar (Duchefa, Haarlem, The Netherlands)) [73] containing $1 \mu \mathrm{M}$ aminoethoxyvinylglycine (AVG) (Sigma, St. Louis, USA). After 3 days, $\sim 100 \mu \mathrm{l}$ of rhizobium LCOs $\left(\sim 10^{-9} \mathrm{M}\right)$ was pipetted on top of the root susceptible zone. After $3 \mathrm{~h}$, roots were fixed in $90 \%$ acetone and subsequently stained for GUS activity. For nodulation assays, compound plants were transferred to perlite and watered with Fåhraeus [74] medium without nitrate. One week after transfer, plants were inoculated with S. meliloti strain $2011\left(O_{600}=0.05-0.1\right)$. For the phosphate starvation experiment, compound plants were transferred to perlite and watered with half-strength Hoagland medium [75]. After one week, plants were removed from perlite and washed three times with demineralized water to get rid of the nutrient salts. Plants were re-planted in fresh perlite and watered with half-strength Hoagland medium with $\left(200 \mu \mathrm{M} \mathrm{PO}_{4}{ }^{3-}\right)$ or without $\left(0 \mu \mathrm{M} \mathrm{PO}_{4}{ }^{3-}\right)$ phosphate, respectively. After 5 days, plants were removed from perlite and stained for GUS activity. 


\section{Histochemical Staining and Microtome Sectioning}

For histochemical GUS staining of $M$. truncatula roots and nodules, samples were first rinsed three times with $100 \mathrm{mM}$ phosphate buffer (PBS, $\mathrm{pH}=7.2$ ). Samples were transferred to GUS-staining buffer (contains $2 \mathrm{mM} \mathrm{K}_{3} \mathrm{Fe}(\mathrm{CN})_{6}, 2 \mathrm{mM} \mathrm{K} \mathrm{Fe}_{4}(\mathrm{CN})_{6}, 10 \mathrm{mM}$ EDTA, 0.1\% Triton X-100 and $1 \mathrm{mg} / \mathrm{ml} \mathrm{X-Gluc} \mathrm{salt} \mathrm{(Duchefa,} \mathrm{Haarlem,} \mathrm{The} \mathrm{Netherlands)} \mathrm{in} 100 \mathrm{mM}$ PBS, pH = 7.2) and placed under vacuum for $30 \mathrm{~min}$. Next, the samples were incubated in the dark at $37^{\circ} \mathrm{C}$ for $3 \mathrm{~h}$. Stained roots were rinsed with PBS $(\mathrm{pH}=7.2)$ three times to stop the reaction.

For historesin embedding, roots and nodule samples were fixed with $5 \%$ glutaraldehyde PBS $(\mathrm{pH}=7.2)$ solution overnight. After fixation, the samples were rinsed with $\mathrm{PBS}(\mathrm{pH}=7.2)$ three times and dehydrated through ethanol gradients (20\%, 40\%,60\%, 80\% and $100 \%$ ). Afterwards, the samples were embedded in Technovit 7100 (Heraeus-Kulzer, Wehrheim, Germany), according to the manufacturer's protocol. GUS-stained samples were sectioned to $7 \mu \mathrm{m}$ using a microtome (Reichert-Jung, Leica Microsystems, Rijswijk, The Netherlands) and stained with $0.1 \%$ Ruthenium Red for 15 min. Images were taken using a Leica DM5500B microscope equipped with a Leica DFC425C camera (Leica Microsystems, Wetzlar, Germany). Images were digitally processed using Photoshop CS6 (Adobe Systems, San Jose, USA).

\section{qRT-PCR Analysis}

RNA was isolated from snap-frozen root material using the plant RNA kit (E.Z.N.A. Omega Biotek, Norcross, USA) following the supplier's manual. cDNA was synthesized from $1 \mu \mathrm{g}$ total RNA using the iScript cDNA synthesis kit (Bio-Rad, Hercules, USA). qRT-PCR reactions were set up in a $20 \mu$ reaction system with $2 \times$ iQ SYBR Green Super-mix (Bio-Rad, Hercules, USA) and the iQ5 Real-time PCR detection system according to the manufacturer's manuals. All primers used in this study were designed using the qPCR settings of Primer3Plus [76]. Relative expression values were calculated using the $2^{-\Delta \Delta C t}$ method, using $M$. truncatula ubiquitin (MtUBQ10) and polypyrimidine tract-binding protein (MtPTB) as reference genes. Statistical significance was determined based on a Students' t-test (unpaired, two tailed, equal variance). All primers used in this study are listed in Supplemental Table 1.

\section{Strigolactone Analysis}

M. truncatula root exudates and root extracts were purified and concentrated as previously described $[17,30]$ with minor modifications. Compound (MtD27 RNAi and empty vector control) plants were grown on perlite and watered twice a week with $50 \mathrm{~mL}$ half-strength $\mathrm{MS}$ medium (Duchefa, Haarlem, The Netherlands). Seven days prior to strigolactone analysis, pots were washed with 3 volumes half-strength $\mathrm{MS}$ medium without $\mathrm{PO}_{4}^{3-}$ (Duchefa, Haarlem, The Netherlands) to initiate phosphate starvation. Strigolactone quantification 
was performed by comparing retention time and mass transitions with those of an available didehydro-orobanchol standard using ultra-performance LC coupled to MS/MS using $\left[{ }^{2} \mathrm{H}_{6}\right] 2$ '-epi-5-deoxystrigol as an internal standard, as previously described [77]. Didehydroorobanchol MS/MS fragmentation spectra of $M$. truncatula were obtained as previously described [17]. Results were subjected to a Students' t-test (unpaired, two tailed, equal variance).

\section{Medicago Gene Atlas IDs}

Probe IDs used for analysis of gene expression using the Medicago gene expression atlas [39] are listed in Supplemental Table 2.

\section{SUPPLEMENTAL DATA}

Supplemental Figures 1-5 are available as Additional Files 1-5 and Supplemental Tables 1-2 are available as Additional Files 6-7 on the BMC Plant Biology website (https://doi. org/10.1186/s12870-015-0651-x).

\section{Author Contributions}

R.G. conceived of the study; R.G., W.C.Y. and T.B. supervised the study; W.K. and A.v.Z. performed functional characterization of MtD27; W.L. and T.T.X. determined the spatiotemporal expression pattern of MtD27; W.L. and A.v.Z. determined rhizobium LCOinduced expression of MtD27; A.v.Z. determined the MtCCD7 and MtCCD8 spatiotemporal expression patterns; T.T.X. and A.v.Z. determined expression of MtD27 in response to phosphate starvation; A.v.Z. and R.G. wrote the manuscript; W.K., W.L., T.T.X., W.C.Y. and T.B. assisted in editing the manuscript.

\section{ACKNOWLEDGMENTS}

We thank Jonathan Kalisvaart and Erik Limpens for their contribution to the project. $\left[{ }^{2} \mathrm{H}_{6}\right] 2^{\prime}-$ epi-5-deoxystrigol was kindly provided by Harro Bouwmeester. This work was supported by a CAS-KNAW Joint PhD Training Program (06PhD12) (WL), NWO-NSFC Joined Research project (846.11.005) (AvZ, WL, WCY, TB and RG), NWO-VICI (865.13.001) (WK, RG), NWOVENI (863.15.010) (WK) and the European Research Council (ERC-2011-AdG294790) (TTX, $\mathrm{TB})$. 


\section{REFERENCES}

1 Soto, M.J. et al. (2010) First indications for the involvement of strigolactones on nodule formation in alfalfa (Medicago sativa). Soil Biol. Biochem. 42, 383-385

2 Foo, E. and Davies, N.W. (2011) Strigolactones promote nodulation in pea. Planta 234, 1073-1081

3 Foo, E. et al. (2013) Strigolactones and the regulation of pea symbioses in response to nitrate and phosphate deficiency. Mol. Plant 6, 76-87

4 Liu, J. et al. (2013) Carotenoid cleavage dioxygenase 7 modulates plant growth, reproduction, senescence, and determinate nodulation in the model legume Lotus japonicus. J. Exp. Bot. 64, 1967-1981

5 De Cuyper, C. et al. (2014) From lateral root density to nodule number, the strigolactone analogue GR24 shapes the root architecture of Medicago truncatula. J. Exp. Bot. 66, 137-146

6 Breakspear, A. et al. (2014) The root hair "infectome" of Medicago truncatula uncovers changes in cell cycle genes and reveals a requirement for auxin signaling in rhizobial infection. Plant Cell 26, 4680-4701

7 Oldroyd, G.E.D. (2013) Speak, friend, and enter: signalling systems that promote beneficial symbiotic associations in plants. Nat. Rev. Microbiol. 11, 252-263

8 Xiao, T.T. et al. (2014) Fate map of Medicago truncatula root nodules. Development 141, 3517-3528

9 Ané, J.-M. et al. (2004) Medicago truncatula DMI1 required for bacterial and fungal symbioses in legumes. Science 303, 1364-1367

10 Lévy, J. et al. (2004) A putative $\mathrm{Ca}^{2+}$ and calmodulin-dependent protein kinase required for bacterial and fungal symbioses. Science 303, 1361-1364

11 Charpentier, M. et al. (2013) The role of DMI1 in establishing $\mathrm{Ca}^{2+}$ oscillations in legume symbioses. Plant Signal. Behav. 8, 8-10

12 Singh, S. et al. (2014) CYCLOPS, a DNA-binding transcriptional activator, orchestrates symbiotic root nodule development. Cell Host Microbe 15, 139-152

13 Kalo, P. et al. (2005) Nodulation signaling in legumes requires NSP2, a member of the GRAS family of transcriptional regulators. Science 308, 1786-1789

14 Smit, P. et al. (2005) NSP1 of the GRAS protein family is essential for rhizobial Nod factor-induced transcription. Science 308, 1789-1791

15 Limpens, E. and Bisseling, T. (2014) CYCLOPS: a new vision on rhizobium-induced nodule organogenesis. Cell Host Microbe 15, 127-129

16 Hirsch, S. et al. (2009) GRAS proteins form a DNA binding complex to induce gene expression during nodulation signaling in Medicago truncatula. Plant Cell 21, 545-557

17 Liu, W. et al. (2011) Strigolactone biosynthesis in Medicago truncatula and rice requires the symbiotic GRAS-type transcription factors NSP1 and NSP2. Plant Cell 23, 3853-3865

18 Maillet, F. et al. (2011) Fungal lipochitooligosaccharide symbiotic signals in arbuscular mycorrhiza. Nature 469, 58-63

19 Lauressergues, D. et al. (2012) The microRNA miR171h modulates arbuscular mycorrhizal colonization of Medicago truncatula by targeting NSP2. Plant J. 72, 512-522

20 Délaux, P. et al. (2013) NSP1 is a component of the Myc signaling pathway. New Phytol. 199, 59-65

21 Lin, H. et al. (2009) DWARF27, an iron-containing protein required for the biosynthesis of strigolactones, regulates rice tiller bud outgrowth. Plant Cell 21, 1512-1525

22 Alder, A. et al. (2012) The path from beta-carotene to carlactone, a strigolactone-like plant hormone. Science 335, 1348-1351

23 Umehara, M. et al. (2008) Inhibition of shoot branching by new terpenoid plant hormones. Nature 455, 195-200

24 Gomez-Roldan, V. et al. (2008) Strigolactone inhibition of shoot branching. Nature 455, 189-194

25 Booker, J. et al. (2005) MAX1 encodes a cytochrome P450 family member that acts downstream of MAX3/4 to produce a carotenoid-derived branch-inhibiting hormone. Dev. Cell 8, 443-449

26 Seto, Y. et al. (2014) Carlactone is an endogenous biosynthetic precursor for strigolactones. Proc. Natl. Acad. Sci. U. S. A. 111, 1640-1645

27 Cardoso, C. et al. (2014) Natural variation of rice strigolactone biosynthesis is associated with the deletion of two MAX1 orthologs. Proc. Natl. Acad. Sci. U. S. A. 111, 2379-2384 
Zhang, Y. et al. (2014) Rice cytochrome P450 MAX1 homologs catalyze distinct steps in strigolactone biosynthesis. Nat. Chem. Biol. 10, 1028-1033

Yoneyama, K. et al. (2007) Phosphorus deficiency in red clover promotes exudation of orobanchol, the signal for mycorrhizal symbionts and germination stimulant for root parasites. Planta 225, 1031-1038 López-Ráez, J.A. et al. (2008) Tomato strigolactones are derived from carotenoids and their biosynthesis is promoted by phosphate starvation. New Phytol. 178, 863-874

Yoneyama, K. et al. (2012) How do nitrogen and phosphorus deficiencies affect strigolactone production and exudation? Planta 235, 1197-1207

Akiyama, K. et al. (2005) Plant sesquiterpenes induce hyphal branching in arbuscular mycorrhizal fungi. Nature 435, 824-827

Kretzschmar, T. et al. (2012) A petunia ABC protein controls strigolactone-dependent symbiotic signalling and branching. Nature 483, 341-344

Yoneyama, K. et al. (2007) Nitrogen deficiency as well as phosphorus deficiency in sorghum promotes the production and exudation of 5-deoxystrigol, the host recognition signal for arbuscular mycorrhizal fungi and root parasites. Planta 227, 125-132

Besserer, A. et al. (2006) Strigolactones stimulate arbuscular mycorrhizal fungi by activating mitochondria. PLoS Biol. 4, 1239-1247

Yoneyama, K. et al. (2008) Strigolactones, host recognition signals for root parasitic plants and arbuscular mycorrhizal fungi, from Fabaceae plants. New Phytol. 179, 484-494

7 Parniske, M. (2008) Arbuscular mycorrhiza: the mother of plant root endosymbioses. Nat. Rev. Microbiol. 6, 763-775

Waters, M.T. et al. (2012) The Arabidopsis ortholog of rice DWARF27 acts upstream of MAX1 in the control of plant development by strigolactones. Plant Physiol. 159, 1073-1085

Benedito, V.A. et al. (2008) A gene expression atlas of the model legume Medicago truncatula. Plant J. 55, 504-513

Heidstra, R. et al. (1994) Root hair deformation activity of nodulation factors and their fate on Vicia sativa. Plant Physiol. 105, 787-797

Journet, E.P. et al. (2001) Medicago truncatula ENOD11: a novel RPRP-encoding early nodulin gene expressed during mycorrhization in arbuscule-containing cells. Mol. Plant Microbe Interact. 14, 737-748

2 Catoira, R. et al. (2000) Four genes of Medicago truncatula controlling components of a nod factor transduction pathway. Plant Cell 12, 1647-1666

Oldroyd, G.E.D. and Long, S.R. (2003) Identification and characterization of nodulation-signaling pathway 2, a gene of Medicago truncatula involved in Nod factor signaling. Plant Physiol. 131, 1027-1032

4 Andriankaja, A. et al. (2007) AP2-ERF transcription factors mediate Nod factor-dependent MtENOD11 activation in root hairs via a novel cis-regulatory motif. Plant Cell 19, 2866-2885

Middleton, P.H. et al. (2007) An ERF transcription factor in Medicago truncatula that is essential for Nod factor signal transduction. Plant Cell 19, 1221-1234

Vijn, I. et al. (1995) Early nodulin gene expression during Nod factor-induced processes in Vicia sativa. Plant J. 8, 111-119

7 Madsen, L.H. et al. (2010) The molecular network governing nodule organogenesis and infection in the model legume Lotus japonicus. Nat. Commun. 1, 10

8 Cerri, M.R. et al. (2012) Medicago truncatula ERN transcription factors: regulatory interplay with NSP1/ NSP2 GRAS factors and expression dynamics throughout rhizobial infection. Plant Physiol. 160, 2155-2172 Deinum, E.E. et al. (2012) Modeling a cortical auxin maximum for nodulation: different signatures of potential strategies. Front. Plant Sci. 3, 96

Mathesius, U. et al. (1998) Auxin transport inhibition precedes root nodule formation in white clover roots and is regulated by flavonoids and derivatives of chitin oligosaccharides. Plant J. 14, 23-34

1 Plet, J. et al. (2011) MtCRE1-dependent cytokinin signaling integrates bacterial and plant cues to coordinate symbiotic nodule organogenesis in Medicago truncatula. Plant J. 65, 622-633

2 Domagalska, M.A. and Leyser, O. (2011) Signal integration in the control of shoot branching. Nat. Rev. Mol. Cell Biol. 12, 211-221

Bennett, T. et al. (2006) The Arabidopsis MAX pathway controls shoot branching by regulating auxin transport. Curr. Biol. 16, 553-563 
54 Crawford, S. et al. (2010) Strigolactones enhance competition between shoot branches by dampening auxin transport. Development 137, 2905-2913

55 Shinohara, N. et al. (2013) Strigolactone can promote or inhibit shoot branching by triggering rapid depletion of the auxin efflux protein PIN1 from the plasma membrane. PLoS Biol. 11, e1001474

56 Sasse, J. et al. (2015) Asymmetric localizations of the ABC transporter PaPDR1 trace paths of directional strigolactone transport. Curr. Biol. 25, 647-655

57 Koltai, H. (2011) Strigolactones are regulators of root development. New Phytol. 190, 545-549

58 Dello loio, R. et al. (2008) Emerging role of cytokinin as a regulator of cellular differentiation. Curr. Opin. Plant Biol. 11, 23-27

59 Marhavy, P. et al. (2011) Cytokinin modulates endocytic trafficking of PIN1 auxin efflux carrier to control plant organogenesis. Dev. Cell 21, 796-804

60 van Zeijl, A. et al. (2015) Rhizobium lipo-chitooligosaccharide signaling triggers accumulation of cytokinins in Medicago truncatula roots. Mol. Plant 8, 1213-1226

61 Wasson, A.P. et al. (2006) Silencing the flavonoid pathway in Medicago truncatula inhibits root nodule formation and prevents auxin transport regulation by rhizobia. Plant Cell 18, 1617-1629

62 Endre, G. et al. (2002) A receptor kinase gene regulating symbiotic nodule development. Nature 417, 962 966

63 Schmutz, J. et al. (2010) Genome sequence of the palaeopolyploid soybean. Nature 463, 178-183

64 Sato, S. et al. (2008) Genome structure of the legume, Lotus japonicus. DNA Res. 15, 227-239

65 Young, N.D. et al. (2011) The Medicago genome provides insight into the evolution of rhizobial symbioses. Nature 480, 520-524

66 Ouyang, S. et al. (2007) The TIGR Rice Genome Annotation Resource: improvements and new features. Nucleic Acids Res. 35, 883-887

67 Tuskan, G.A. et al. (2006) The genome of black cottonwood, Populus trichocarpa (Torr. \& Gray). Science 313, 1596-1604

68 Jaillon, O. et al. (2007) The grapevine genome sequence suggests ancestral hexaploidization in major angiosperm phyla. Nature 449, 463-467

69 Katoh, K. et al. (2002) MAFFT: a novel method for rapid multiple sequence alignment based on fast Fourier transform. Nucleic Acids Res. 30, 3059-3066

70 Huelsenbeck, J.P. and Ronquist, F. (2001) MRBAYES: Bayesian inference of phylogenetic trees. Bioinformatics 17, 754-755

71 Limpens, E. et al. (2005) Formation of organelle-like $\mathrm{N}_{2}$-fixing symbiosomes in legume root nodules is controlled by DMI2. Proc. Natl. Acad. Sci. U. S. A. 102, 10375-10380

72 Limpens, E. et al. (2004) RNA interference in Agrobacterium rhizogenes-transformed roots of Arabidopsis and Medicago truncatula. J. Exp. Bot. 55, 983-992

73 Ehrhardt, D.W. et al. (1992) Depolarization of alfalfa root hair membrane potential by Rhizobium meliloti Nod factors. Science 256, 998-1000

74 Fåhraeus, G. (1957) The infection of clover root hairs by nodule bacteria studied by a simple glass slide technique. J. Gen. Microbiol. 16, 374-381

75 Hoagland, D.R. and Arnon, D.I. (1950) The water-culture method for growing plants without soil. Circ. Calif. Agric. Exp. Stn. 347, 1-32

76 Untergasser, A. et al. (2007) Primer3Plus, an enhanced web interface to Primer3. Nucleic Acids Res. 35, W71-W74

77 Kohlen, W. et al. (2011) Strigolactones are transported through the xylem and play a key role in shoot architectural response to phosphate deficiency in nonarbuscular mycorrhizal host Arabidopsis. Plant Physiol. 155, 974-987 



\section{Chapter 5}

CRISPR/Cas9-mediated mutagenesis

of four putative symbiosis genes of

the tropical tree Parasponia andersonii

reveals novel phenotypes

Arjan van Zeijl, Titis Wardhani, Maryam Seifi-Kalhor, Luuk Rutten, Fengiiao Bu, Marijke Hartog, Sidney Linders, Elena Fedorova, Ton Bisseling, Wouter Kohlen and Rene Geurts 


\section{Abstract}

Parasponia represents five fast growing tropical tree species in the Cannabeaceae and is the only plant lineage besides legumes that can establish nitrogen-fixing nodules with rhizobium. Comparative analyses between legumes and Parasponia allows identification of conserved genetic networks controlling this symbiosis. However, such analyses are currently hampered due to the absence of powerful reverse genetic tools for Parasponia. Here, we present a fast and efficient protocol for CRISPR/Cas9 mutagenesis in Agrobacterium tumefaciens-transformed Parasponia andersonii plants. Using this protocol, mutants can be obtained within three months. Due to efficient micro-propagation, bi-allelic mutants can be studied in the $T_{0}$ generation, allowing phenotypic evaluation within six months after transformation. As proof of principle, we mutated four genes - PanHK4, PanEIN2, PanNSP1, and PanNSP2 - that control cytokinin, ethylene or strigolactone hormonal networks and that in legumes commit essential symbiotic functions. Knockout mutants in Panein2 and Panhk4 displayed developmental phenotypes that are associated with ethylene or cytokinin signaling mutations. Like in legumes, NSP GRAS-type transcriptional regulators showed to be essential for nodule formation in P. andersonii. Conversely, symbiotic phenotypes of Panhk4 and Panein2 mutants differ from that in legumes. This illustrates the value of Parasponia trees as research model for reverse genetic studies. 


\section{INTRODUCTION}

Parasponia are tropical tree species belonging to the Cannabis family (Cannabaceae) and are known as the only non-legume plants that can establish a nitrogen-fixing endosymbiosis with rhizobium [1-3]. The Parasponia genus consists of five species indigenous to the Malay Archipelago and Papua New Guinea, where they grow on the slopes of volcanic mountains $[2,4,5]$. Parasponia spp. are typical fast-growing pioneer plants, capable of covering nitrogen-poor eroded soils in a relatively short time span [4]. Under suitable greenhouse conditions, young Parasponia trees can grow at speeds exceeding 45 centimeters per month, and fix up to $850 \mathrm{~kg} \mathrm{~N} \mathrm{ha}^{-1}$ year $^{-1}$ in association with rhizobium [6-8]. Therefore, Parasponia could represent a valuable tool for studies aimed at understanding the genetics underlying rhizobium symbiosis as well as tree biology [9-13].

Like legumes, Parasponia develops specialized root nodular organs to host the rhizobium partner. Nodules provide the rhizobium bacteria with suitable environmental conditions to convert atmospheric nitrogen into ammonium. The Cannabaceae and legume families (Fabaceae) diverged about a hundred million years ago [14], underlining that the rhizobium symbiosis in legumes and Parasponia evolved largely independent [15]. This is reflected in the distinct nodule-types found in both lineages [9]. Legume nodules possess a large central zone of infected cells that is surrounded by peripheral vascular bundles. In contrast, Parasponia nodules have a central vascular bundle and infected cells in the peripheral zone, giving these nodules a lateral root-like appearance. Nevertheless, initial comparative studies revealed that both symbioses are founded on conserved signaling networks. In legumes as well as Parasponia, root nodule formation is induced upon recognition of rhizobial secreted lipo-chitooligosaccharide (LCO) signals [16-18]. Research on model legumes, like Medicago truncatula and Lotus japonicus, showed that the perception of these symbiotic signals requires a signaling cascade that has been largely co-opted from the much older endomycorrhizal symbiosis $[10,19]$. In legumes, activation of the LCO signaling network results in a massive transcriptional reprogramming, requiring among others the GRAStype transcriptional regulators NODULATION SIGNALLING PATHWAY 1 (NSP1) and NSP2 and the cytokinin receptor MtCRE1/LjLHK1 [20-26]. Subsequent nodule formation is tightly controlled by regulatory feedback loops, including negative regulation by ethylene signaling [27-29].

A reference quality genome sequence for Parasponia andersonii and draft genome sequences of two additional Parasponia species have been generated [30]. Mining these genomes uncovered $\sim 1,800$ putative symbiosis genes, of which hundreds are close homologs 
of legume symbiosis genes [30]. Initial reverse genetic studies in $P$. andersonii, using a transient Agrobacterium rhizogenes-based root transformation system, revealed that at least two genes - NOD FACTOR PERCEPTION 1 (PANNFP1) and CALCIUM AND CALMODULINDEPENDENT PROTEIN KINASE (PAnCCAMK) - commit conserved functions in the Parasponia and legume LCO signaling pathways [18]. We argue that a more comprehensive comparative analysis between legumes and Parasponia will allow identification of conserved genetic networks that are essential to establish symbiosis with rhizobium. However, to use Parasponia as an effective research model - alongside the legume models $M$. truncatula and L. japonicus - efficient transformation and genome editing tools are required.

Here, we exploit an efficient in vitro micro-propagation system available for $P$. andersonii to establish stable transformation and CRISPR/Cas9-mediated mutagenesis for this species [31-33]. We show that using Agrobacterium tumefaciens-mediated transformation, stable transgenic lines of $P$. andersonii can be obtained in 3-4 months. Additionally, we show that $P$. andersonii is amenable to targeted mutagenesis using the CRISPR/Cas9 system. As $\sim 40 \%$ of the resulting $T_{0}$ lines harbor bi-allelic mutations, these can be phenotyped upon in vitro propagation. As proof of concept, we mutated four genes in $P$. andersonii that in legumes control hormonal pathways as well as commit symbiotic functions. These include, the GRAS-type transcriptional regulators NSP1 and NSP2 that both are essential for nodule organogenesis $[21,22,25]$ as well as control strigolactone biosynthesis by mediating DWARF27 (D27) expression [34, 35]; HISTIDINE KINASE 4 (HK4), which encodes a cytokinin receptor that in legumes is essential for nodule organogenesis [20, 23, 24]; ETHYLENE INSENSITIVE 2 (EIN2), which is an essential hub in ethylene signaling and a negative regulator of nodulation in legumes $[27,28,36]$. The corresponding $P$. andersonii mutants display hormone-related phenotypes. However, the symbiotic phenotypes of Panhk4 and Panein2 mutants deviate from those described for legumes. This illustrates that $P$. andersonii can be used as an effective model for CRISPR/Cas9-mediated reverse genetic studies.

\section{Results}

\section{Agrobacterium tumefaciens-Mediated Transformation of Parasponia}

To establish a protocol for stable transformation of $P$. andersonii, we first determined the most optimal conditions for regeneration of non-transgenic tissue. We compared regeneration efficiencies of nine different tissue explant types in combination with eleven different media, including the propagation and root-inducing media previously used for P. andersonii (Supplemental Tables 1-2) $[18,31]$. This revealed that young stem pieces and petioles placed on original propagation medium regenerate plantlets most efficiently 

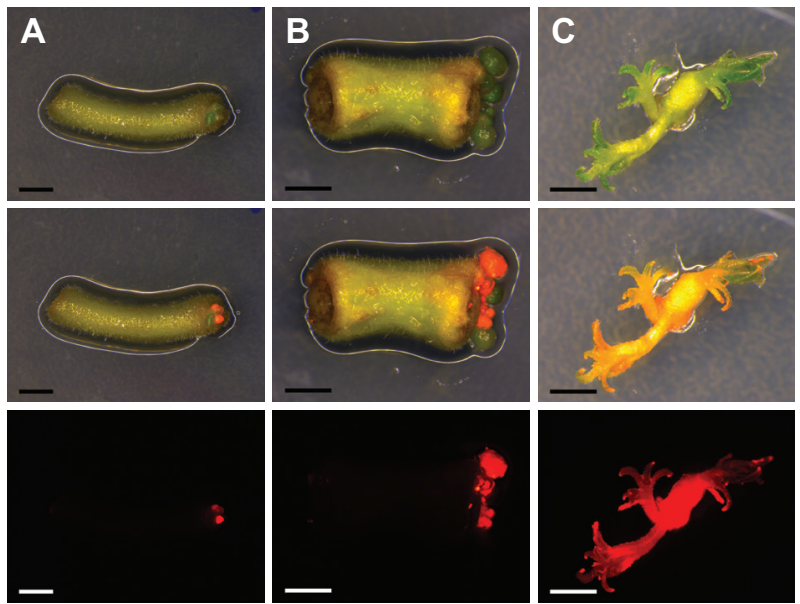

Figure 1. P. andersonii stem and petiole explants can be efficiently transformed using Agrobacterium tumefaciens.

(A) Petiole explant at four weeks after transformation using A. tumefaciens.

(B) Stem explant at five weeks after transformation using A. tumefaciens.

(C) Small transgenic shoots at ten weeks after transformation.

Explants were incubated on root-inducing medium for nine days, prior to transfer to propagation medium. DsRED fluorescence indicates transgenic tissue. Scale bars are equal to $2.5 \mathrm{~mm}$. Shown from top to bottom are bright-field images, overlays of bright-field and DsRED fluorescence and DsRED fluorescence images.

(Supplemental Table 1). Next, we questioned whether stem pieces and petioles could be effectively transformed using $A$. tumefaciens. To this end, we used $A$. tumefaciens AGL1 carrying a binary transformation vector containing in its T-DNA the kanamycin resistance gene NPTII and the red fluorescent protein DsRED1. Co-cultivation of $A$. tumefaciens and $P$. andersonii stem or petiole explants was conducted in darkness for two days at $21^{\circ} \mathrm{C}$ to promote T-DNA transfer [31]. Afterwards, tissue explants were placed on selective medium and incubated at $28^{\circ} \mathrm{C}$ in the light. These latter conditions are most favorable for $P$. andersonii regeneration [31]. From day eight onwards, DsRED1-fluorescent cells could be observed indicating successful transfer of the T-DNA.

Recent research on Arabidopsis thaliana showed that acquisition of pluripotency requires activation of a root developmental program [37]. We tested whether an initial culturing period on root-inducing medium further improves the transformation efficiency of $P$. andersonii. This showed to be the case (Supplemental Table 3). About half of the explants formed regenerative calli at four weeks post co-cultivation (Figure 1A). When $2 \mathrm{~mm}$ in size, transgenic calli were separated from tissue explants, which stimulated shoot formation 
(Figure 1B-C). Two to three months after start of transformation, a single shoot was selected from each explant to ensure that selected transgenic lines represent independent transformation events. These shoots can be genotyped and vegetatively propagated (Supplemental Figure 1).

To characterize the resulting transgenic $P$. andersonii lines at the molecular level, we selected - based on red fluorescence - twenty independent transformants for further analyses. PCR reactions using primers amplifying a sequence near the right T-DNA border indicated complete T-DNA integration in 19 out of 20 lines (Supplemental Table 4). To determine whether the transformation procedure might affect ploidy level of the regenerated transgenic lines, we estimated genome size based on flow cytometry. This showed no effect of the transformation procedure on genome size of transgenic lines (Supplemental Table 4). To estimate the number of T-DNA integrations, we used quantitative RT-PCR (qRT-PCR) as well as Southern blotting. This showed an overall low T-DNA copy number, varying between one and three integrations per line (Supplemental Table 4). We selected three transgenic lines with a single T-DNA integration to examine T-DNA stability. In greenhouse-grown trees as well as in vitro propagated material, DsRED1 fluorescence could still be observed at one year after transgenic lines were selected (Supplemental Figure 2). This indicates that transgenes remain stably integrated in the $P$. andersonii genome and actively transcribed, even after multiple rounds of vegetative propagation. Taken together, the protocol described above allows to generate $A$. tumefaciens-transformed $P$. andersonii plantlets within three months, which upon vegetative propagation can be phenotyped.

\section{Parasponia Is Amenable to CRISPR/Cas9-Mediated Mutagenesis}

To test whether CRISPR/Cas9 could be used for targeted mutagenesis in P. andersonii, we aimed at mutating the $P$. andersonii putative orthologues of EIN2, MtCRE1/LjLHK1, NSP1 and NSP2. These genes were selected, because they control legume root nodule formation as well as commit essential non-symbiotic functions in hormone homeostasis. Putative orthologues of all four genes were previously identified from the $P$. andersonii genome and named PanEIN2, PanHK4, PanNSP1 and PanNSP2, respectively [30]. Phylogenetic reconstruction based on protein sequences confirmed that these represent the most likely orthologues of legume symbiotic genes (Supplemental Figures 3-6). To mutate PanEIN2, PanHK4, PanNSP1 and PanNSP2, three single guide RNAs (sgRNAs) targeting PanHK4 and PanNSP2 and single sgRNAs targeting PanEIN2 and PanNSP1 were placed under an $A$. thaliana AtU6 small RNA promoter [38]. These were cloned into a binary transformation vector containing the NPTII kanamycin resistance gene as well as a Cas9-encoding sequence 
Table 1. Mutation frequency in CRISPR/Cas9 transgenic lines.

\begin{tabular}{|c|c|c|c|c|c|c|}
\hline \multirow{2}{*}{$\begin{array}{l}\text { Target } \\
\text { gene }\end{array}$} & \multirow{2}{*}{$\begin{array}{c}\text { No. of } \\
\text { sgRNA's }\end{array}$} & \multirow{2}{*}{$\begin{array}{l}\text { No. of } \\
\text { lines }\end{array}$} & \multirow{2}{*}{$\begin{array}{c}\text { Non- } \\
\text { mutated }^{1}\end{array}$} & \multicolumn{3}{|c|}{ Mutated } \\
\hline & & & & Bi-allelic & Heterozygous & Unknown ${ }^{2}$ \\
\hline$N S P 1$ & 1 & 29 & 15 (51.7\%) & 11 (37.9\%) & 0 & $3(10.3 \%)$ \\
\hline NSP2 & 3 & 29 & $13(44.8 \%)$ & $10(34.5 \%)$ & 3 (10.3\%) & $3(10.3 \%)$ \\
\hline EIN2 & 1 & 9 & 1 (11.1\%) & $6(66.7 \%)$ & $2(22.2 \%)$ & 0 \\
\hline$H K 4$ & 3 & 26 & $13(50.0 \%)$ & $12(46.2 \%)$ & $1(3.8 \%)$ & 0 \\
\hline Total & & 93 & $42(45.2 \%)$ & 39 (41.9\%) & $6(6.5 \%)$ & $6(\%)$ \\
\hline
\end{tabular}

fused to an N-terminal nuclear-localization signal and driven by the CaMV 355 promoter $[39,40]$. The resulting constructs as well a control construct containing only the NPTII- and Cas9-encoding sequences were transformed to $P$. andersonii using the method described above. For all constructs, transgenic shoots were obtained, although in case of the construct targeting PanHK4 regeneration took considerably longer (up to six months). Genotyping of regenerated shoots showed that $>85 \%$ contained the Cas 9 gene, indicating successful transformation. Potential mutations at any of the target sites were identified through PCR amplification and subsequent sequencing of the PCR product. This revealed mutations at the target site in about half of the transgenic shoots examined, of which the majority were bi-allelic (Table 1). Most mutations represent small insertion and deletions but also larger deletions and inversions were identified, some of which occur in between two target sites (Supplemental Figures 7-10). In case of PanHK4, most mutants contained small in-frame deletions of three or six base pairs that most likely do not disrupt protein function. In fact, only two bi-allelic knockout mutants could be identified (Supplemental Figure 8). For the remaining three constructs, multiple bi-allelic knockout mutants were identified of which three individuals were selected for further studies (for an overview of mutant alleles see Supplemental Figures 7-10).

For phenotypic evaluation, $P$. andersonii $\mathrm{T}_{0}$ transgenic lines are propagated vegetatively. Therefore, we first evaluated whether any of the mutant lines might be chimeric. To this end, tissue samples were taken from at least three different positions and genotyped for the corresponding target mutation. For each of the mutant lines, except Pannsp2-9, the exact same mutations were retrieved, suggesting that genome editing occurred soon after T-DNA integration. In case of Pannsp2-9, chimeric mutations were detected at the first of three target sites (Supplemental Figure 10C). However, the nature of the mutations at the second 
A

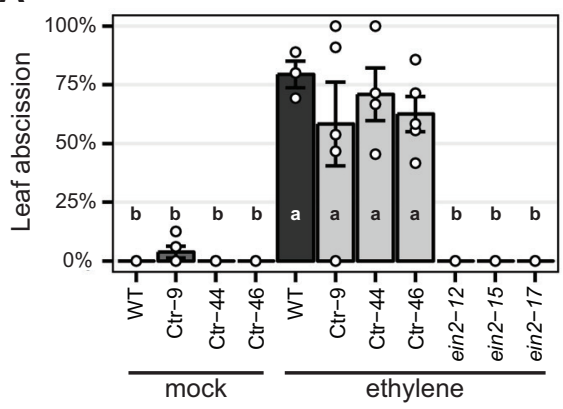

B

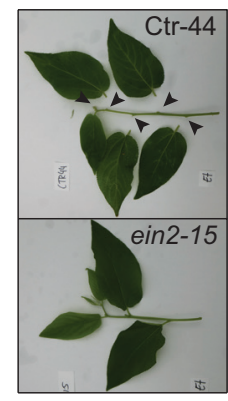

Figure 2. Panein2 mutants are insensitive to ethylene treatment.

(A) Percentage of abscised leaves after mock or ethylene treatment. Data represent means of 3-5 biological replicates \pm SEM. Dots represent measurement values of biological repeats. Different letters indicate statistical significance $(p<0.05)$ as determined by ANOVA in combination with Tukey post-hoc test.

(B) Representative images showing abscission of leaves on a transgenic control line, but not on a Panein2 mutant. Abscission points are indicated by arrowheads.

and third target site prevent that gene function could be restored in this line. Therefore, all eleven mutants are suitable for phenotypic evaluation. This proves that CRISPR/Cas9 can be used to efficiently mutagenize $P$. andersonii in the $\mathrm{T}_{0}$ generation.

\section{Non-Symbiotic Phenotypes in Parasponia ein2, hk4, nsp1 and nsp2 Mutant Lines}

To characterize the resulting Panein2, Panhk4, Pannsp1 and Pannsp2 mutant lines, we studied their non-symbiotic phenotypes. PanEIN2 putatively encodes a central component of the ethylene signaling pathway and therefore Panein 2 mutants are expected to be ethylene insensitive. One phenotype triggered in response to ethylene treatment is abscission of leaves and flowers [41-43]. We exploited this phenotype to assess ethylene sensitivity of Panein2 mutants. To this end, the tips of young shoot branches of greenhouse grown trees were exposed to ethylene gas. Within three days, ethylene triggered abscission of $\sim 65 \%$ of treated leaves on wild-type $P$. andersonii as well as control transgenic lines (Figure 2). In contrast, leaf abscission was not observed on Panein2 mutant trees (Figure 2). This demonstrates that Panein 2 mutants are indeed ethylene insensitive.

Inspection of Panein2 mutant trees revealed an additional non-symbiotic phenotype. These trees form bisexual flowers containing both male and female reproductive organs (Figure 3A-C). In contrast, wild-type $P$. andersonii trees form unisexual flowers that contain either stamens or carpels [4] (Figure 3D-E). This suggests that ethylene is involved in the regulation of Parasponia sex type. 


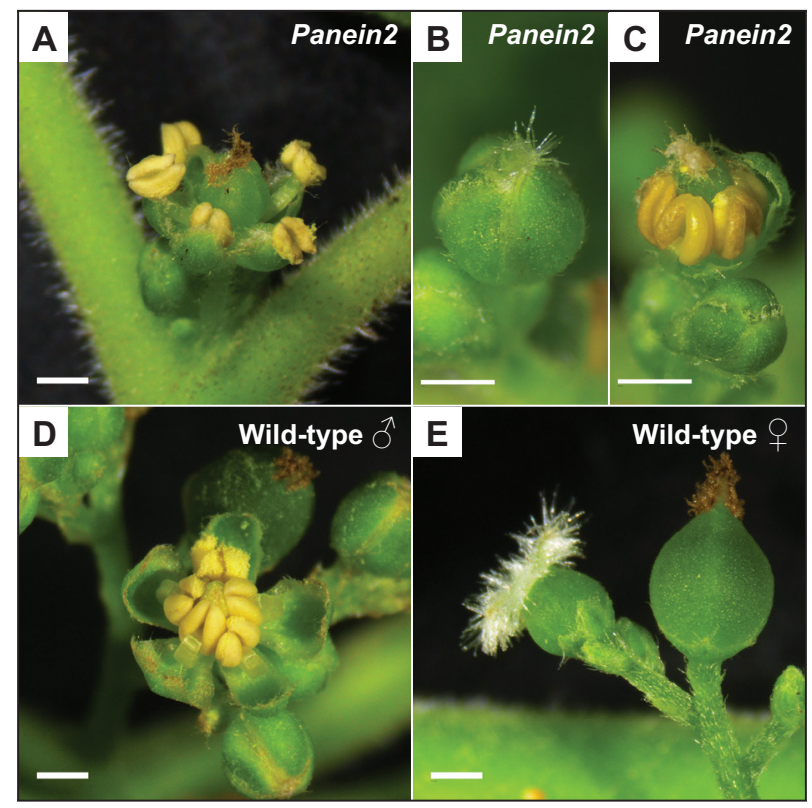

Figure 3. Panein2 mutants form bisexual flowers.

(A) Mature Panein2 flower. Note the presence of both stamen and a carpel inside Panein2 flowers.

(B) Immature Panein2 flower. Note the presence of stigmata, indicating presence of a carpel inside the flower.

(C) Immature Panein2 flower of which sepals have been removed to show the presence of stamen.

(D) Mature wild-type male flower.

(E) Mature wild-type female flowers. Left, young female flower. Right, older female flower.

Scale bars are equal to $1 \mathrm{~mm}$.

Cytokinins are important regulators of cambial activity [44-46]. To determine whether reduced cytokinin sensitivity in Panhk4 mutant lines affects activity of the procambium, we sectioned the base of young axillary shoot branches. This showed that cambium activity is reduced in Panhk4 mutant lines compared to transgenic controls (Figure 4). Therefore, we conclude that PanHK4-mediated cytokinin signaling is required for regulation of $P$. andersonii secondary growth.

Expression studies in $M$. truncatula, previously identified a set of genes downregulated in roots of Mtnsp1 and Mtnsp2 mutants [34]. Among these are DWARF27 (MtD27) (Medtr1g471050) and MORE AXILLARY BRANCHING 1 (MtMAX1) (Medtr3g104560) that are putatively involved in strigolactone biosynthesis [34, 35, 47, 48]. We identified putative $P$. andersonii orthologues of these genes (Supplemental Figures 11-12) and compared their expression levels in young root segments of three Pannsp1, Pannsp2 and control plants 


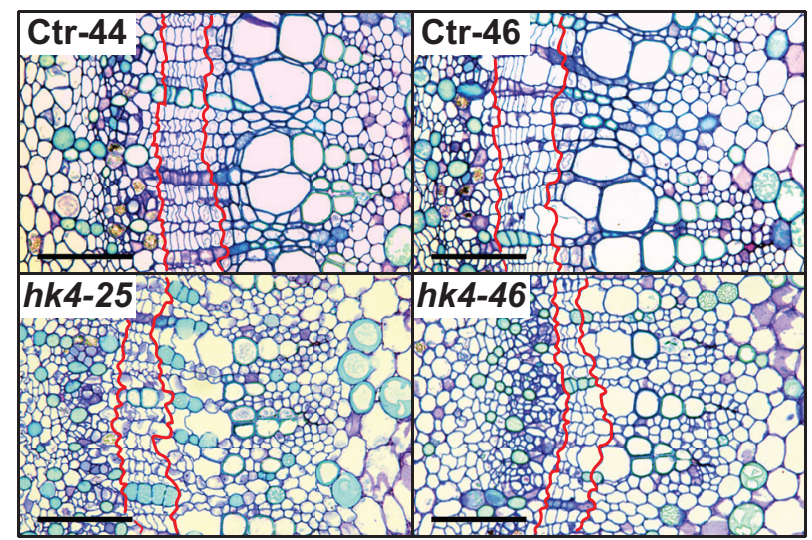

Figure 4. Panhk4 mutants display reduced cambial activity.

Stem cross-sections of control transgenic and Panhk4 mutant lines. Sections were made of the base of the second youngest branch of four month old trees grown under greenhouse conditions. The location of the procambium is indicated by red lines. Scale bars represent $100 \mu \mathrm{m}$.

by qRT-PCR. This showed that expression of PanD27 and PanMAX1 is reduced in roots of Pannsp1 and Pannsp2 mutant lines (Figure 5). We noted that Pannsp1 mutant lines differ in the level of PanD27 and PanMAX1 expression. Both genes have an intermediate expression level in Pannsp1-6 and Pannsp1-13, compared to Pannsp1-39 and Pannsp2 mutants (Figure 5). The three Pannsp1 mutant lines differ from each other in the type of mutations that were created. Pannsp1-6 and Pannsp1-13 contain a one base pair insertion and five base pair deletion close to the 5 '-end of the coding region, respectively. These mutations are immediately followed by a second in-frame ATG that in wild-type PanNSP1 encodes a methionine at position 16. In contrast, Pannsp1-39 contains a large 232 base pair deletion that removes this in-frame ATG (see Supplemental Figure 9). Together, this suggests that Pannsp1-6 and Pannsp1-13 might represent weak alleles. Overall, these data suggest that regulation of $D 27$ and MAX1 expression by NSP1 and NSP2 is conserved between $M$. truncatula and $P$. andersonii.

Taken together, we showed that EIN2, HK4, NSP1 and NSP2 in P. andersonii commit nonsymbiotic functions in hormonal homeostasis. These functions are in line with what is described for other plant species, suggesting that the generated $P$. andersonii lines represent true mutants. 

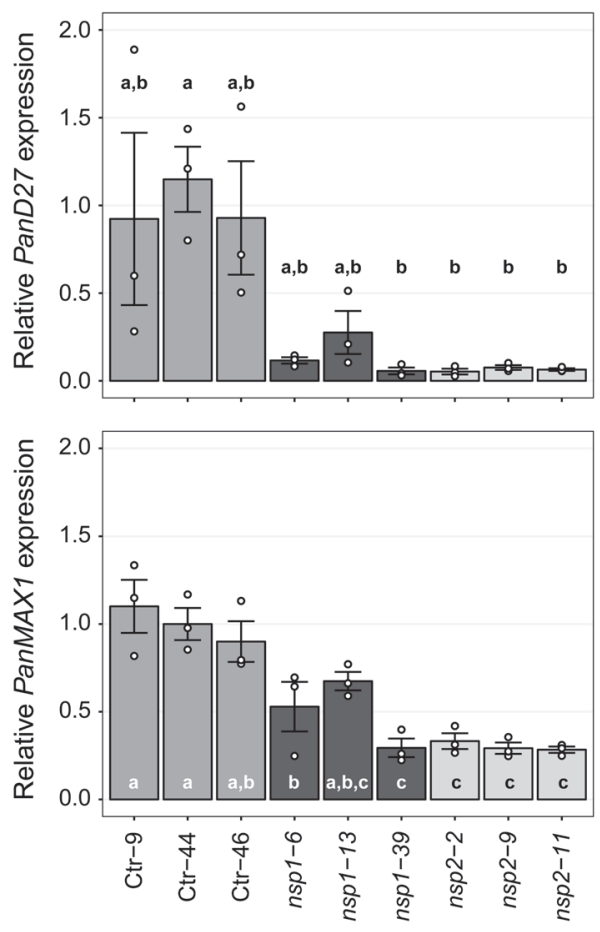

Figure 5. Expression of PanD27 and PanMAX1 is reduced in Pannsp1 and Pannsp2 mutant roots.

Relative expression of PanD27 (A) and PanMAX1 (B) in roots of transgenic control and Pannsp1 and Pannsp2 mutant lines. Data represent means of three biological replicates \pm SEM. Dots represent measurement values of biological repeats. Different letters indicate statistical significance $(p<0.05)$ as determined by ANOVA in combination with Tukey post-hoc test.

\section{Nodulation Phenotypes of Parasponia Panein2 and Panhk4 Mutants Differ from Their Legume Counterparts}

To determine whether PanEIN2, PanHK4, PanNSP1 and PanNSP2 perform similar functions during nodule formation as their legume orthologous, $P$. andersonii mutant plantlets were inoculated with Mesorhizobium plurifarium BOR2 [30]. Nodulation phenotypes were examined one month post inoculation.

The strong Pannsp1-39 mutant allele and all three Pannsp2 mutant lines are unable to form root nodules (Figure 6A and Supplemental Figure 13). This is similar as described for M. truncatula, L. japonicus and Pisum sativum nsp1 and nsp2 mutants [21, 22, 25, 49]. In contrast, the weak Pannsp1 alleles Pannsp1-6 and Pannsp1-13 could be nodulated similar as 
A
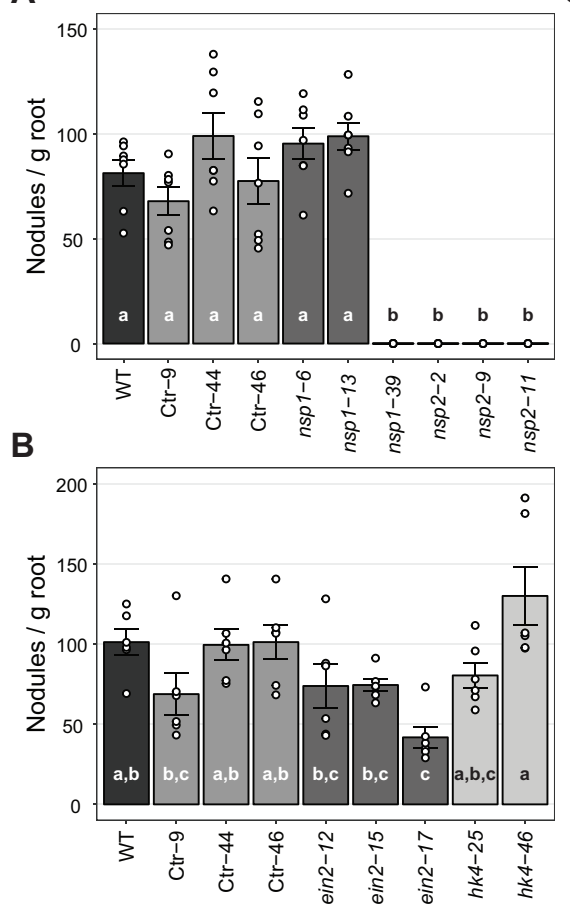

C

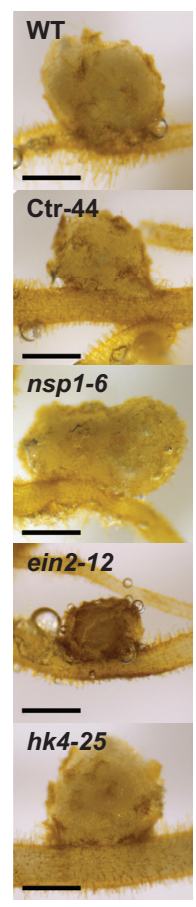

Figure 6. Nodule formation on $P$. andersonii CRISPR/Cas9 mutant lines.

(A) Nodule number per gram root fresh weight on wild type (WT), transgenic control and Pannsp1 and Pannsp2 mutant lines. Nodule number was determined at one month after inoculation with Mesorhizobium plurifarium BOR2.

(B) Nodule number per gram root fresh weight on wild type (WT), transgenic control and Panein2 and Panhk4 mutant lines. Nodule number was determined at one month after inoculation with Mesorhizobium plurifarium BOR2.

(C) Representative images of one month-old nodules. Note the dark color of Panein2 nodules. Scale bars are equal to $0.5 \mathrm{~mm}$.

Data in (A-B) represent means of 5-7 biological replicates \pm SEM. Dots represent measurement values of biological repeats. Different letters indicate statistical significance $(p<0.05)$ as determined by ANOVA in combination with Tukey post-hoc test. Data on nodule number and root weight are shown in Supplemental Figure 13.

wild-type or control transgenic plants (Figure 6A and Supplemental Figure 13), suggesting that residual PanNSP1 activity is sufficient to support root nodule formation. Overall, these data show that NSP1 and NSP2 functioning is essential for root nodule formation in Parasponia. 
Analysis of the nodulation phenotype of $P$. andersonii Panhk4 mutants showed that PanHK4 is not required for root nodule formation. Both Panhk4 mutant lines formed a similar amount of nodules as wild type and transgenic controls (Figure 6B and Supplemental Figure 13). This is different from the corresponding legume mutants - M. truncatula Mtcre 1 and $L$. japonicus Ljlhk1 - that generally are not forming root nodules [23, 24].

The phenotype of $P$. andersonii Panein2 mutants also differs from that of legume mutants. M. truncatula ein2 mutants - as well as L. japonicus plants in which both EIN2-encoding genes have been silenced - form more nodules than wild type, which are clustered in distinct zones along the root [27, 36]. Panein2 mutants do not form such nodule clusters and nodule number is not higher than wild type (Figure 6B and Supplemental Figure 13). However, nodules formed on Panein 2 mutant plants are generally smaller and dark colored when compared to nodules of control plants (Figure $6 \mathrm{C}$ ). This suggests impaired nodule development in $P$. andersonii ein2 mutants.

To determine the cyto-architecture of Panein2, Panhk4 and Pannsp1-6/Pannsp1-13 mutant nodules, we sectioned $\sim 10$ nodules for each mutant line and studied these by light microscopy. Wild-type $P$. andersonii nodules harbor an apical meristem, followed by several cell layers that contain infection threads (Figure 7A) [50]. Below this infection zone, 2-3 cell layers are found that display vacuolar fragmentation and increase in size compared to noninfected cells (Figure 7B). These cells are immediately followed by cells that are fully filled with fixation threads (Figure 7B). The general cyto-architecture of Panhk4 and Pannsp1-6/ Pannsp1-13 mutant nodules does not differ from that of wild-type or transgenic control nodules (Figure 7A, C-D), suggesting that these are fully functional. In contrast, in Panein2 mutant nodules intracellular infection is hampered (Figure 7E-H). Most (> 75\%) Panein2 mutant nodules harbor only infection threads as well as large apoplastic colonies (Figure 7H). Some mutant nodules, harbor cells that contain fixation threads. However, even in the best nodules, fixation thread formation is severely delayed and many cells in the fixation zone still show vacuolar fragmentation (Figure $\mathbf{7 H}$ ). This shows that ethylene signaling is required for efficient fixation thread formation in $P$. andersonii nodules.

Taken together, these data reveal symbiotic mutant phenotypes for nsp1, nsp2 and ein2, whereas no effect on nodule formation was found by knocking out $h k 4$ in $P$. andersonii. Interestingly, we uncovered a novel role for the ethylene signaling component EIN2 in intracellular infection of $P$. andersonii nodules. 


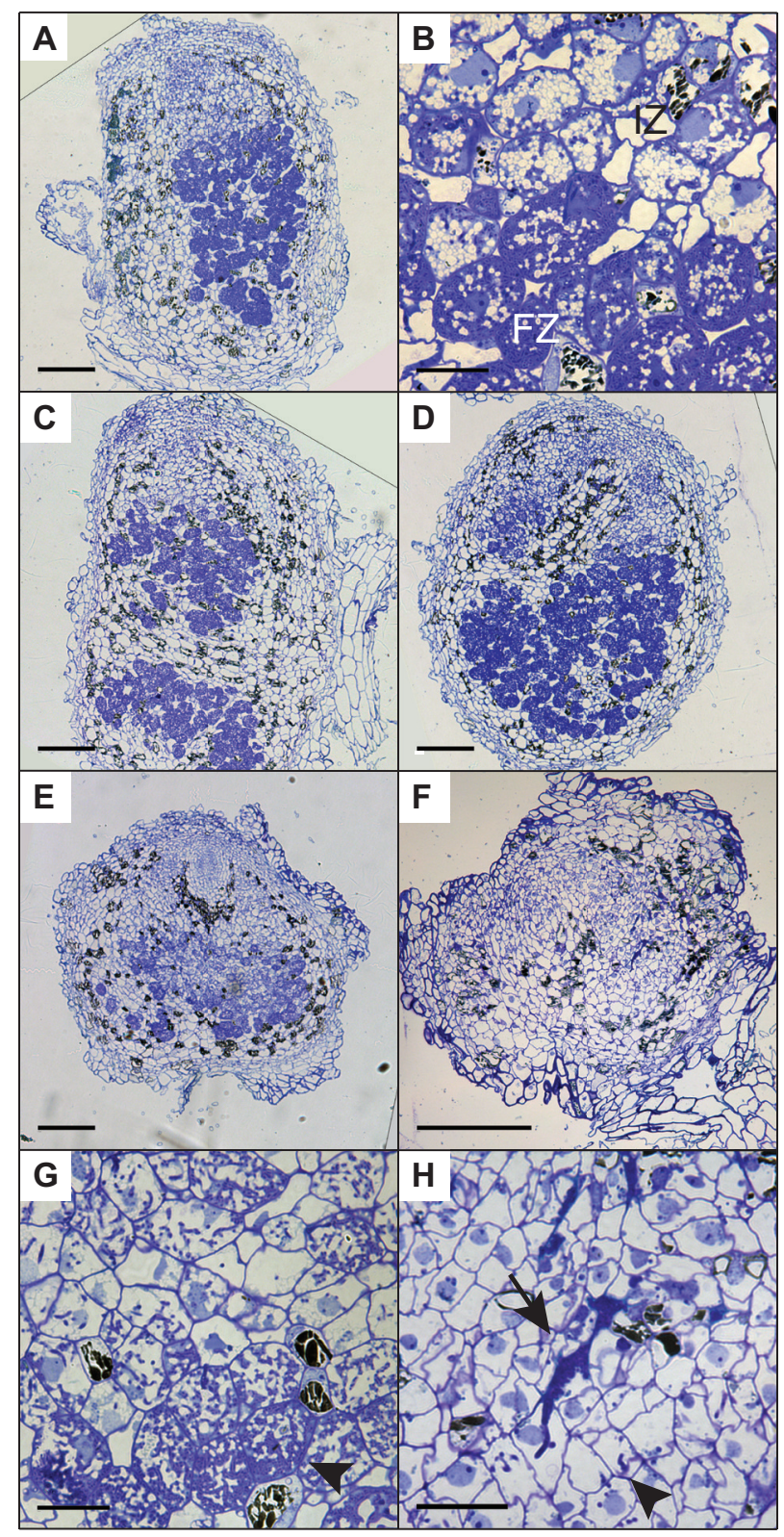

Figure 7. Cyto-architecture of CRISPR/Cas9 mutant nodules.

Longitudinal nodule sections of one month-old nodules formed on transgenic control line Ctr-9 (A), Pannsp1-13 (C), Panhk4-25 (D), Panein2-15 (E) and Panein2-17 (F).

(B) Zoom in of cells in the infection (IZ) and fixation zone (FZ) of a transgenic control nodule shown in (A). Note the presence of small fragmented vacuoles in infected cells in the infection zone. 


\section{Discussion}

Comparative studies between legumes and the Cannabaceae tree Parasponia can provide insights in 'core' genetic networks underlying rhizobium symbiosis [30]. To facilitate such studies, we aimed to establish a reverse genetics platform for $P$. andersonii based on CRISPR/ Cas9 genome editing. We show that using $A$. tumefaciens transformation, $P$. andersonii stable transgenic lines can be obtained in 3-4 months. In combination with CRISPR/Cas9 mutagenesis, this allows efficient generation of bi-allelic knockout mutants. As proof-ofconcept, we mutated four genes that commit essential symbiotic functions in legumes as well as control different hormonal networks. Characterization of the resulting lines revealed both symbiotic as well as non-symbiotic mutant phenotypes. Therefore, we conclude that stable $A$. tumefaciens-mediated transformation in combination with CRISPR/Cas9 genome editing can be efficiently used for reverse genetic analysis in $P$. andersonii.

Plant transformation efficiency is the main bottleneck in plant genome editing [51, 52]. Especially regeneration of an entire transgenic plant out of a single transformed cell remains difficult for most plant species. We took advantage of an efficient micro-propagation system available for Parasponia spp. to establish a protocol for stable transformation [3133]. About 8-12 weeks after cocultivation with A. tumefaciens, $~ 50 \%$ of explants develop transgenic shoots. This relatively high efficiency is, in part, obtained through an initial nineday culturing period on root-inducing medium, prior to incubation on standard propagation medium. This adaptation in the protocol was inspired by a recent study that showed that regeneration of plant cells consists of two distinctive steps [37]. Regenerative competence is established through activation of a root developmental program, followed by activation of shoot promoting factors that are required to complete shoot regeneration [37]. The latter explains why transfer to propagation medium is required to regenerate $P$. andersonii transgenic shoots. However, this promoting effect of rooting medium on regeneration of transgenic shoots might differ between different explant types, as noted for $P$. andersonii stems and petioles (Supplemental Table 3).

(G) Zoom in on cells in the basal part of the Panein2-15 nodule shown in (E). Indicated by an arrowhead are cells containing fixation threads.

(H) Zoom in of the Panein2-17 nodule shown in (F). Indicated by an arrowhead are infection threads. Indicated by an arrow are large apoplastic colonies.

Scale bars in (A, C-F) are equal to $150 \mu \mathrm{m}$ and $25 \mu \mathrm{m}$ in (B, G-H). 
An advantage of the Parasponia system is that $\mathrm{T}_{0}$ transgenic knockout mutants can be clonally propagated through in vitro micro-propagation [31-33]. This allows a large number of rooted plantlets to be generated in a relatively short time span. As a result, phenotypic characterization can be initiated already at four months after start of transformation. However, a disadvantage of clonal propagation in combination with CRISPR/Cas 9 mutagenesis is the possibility of obtaining chimeric mutants. Among the mutant lines we created, we identified one line (out of twelve) that was chimeric for one out of three CRISPR target sites (Supplemental Figure 10C). Most mutant lines were genetically homogeneous, suggesting that mutations are generally induced soon after T-DNA integration. This is consistent with results in poplar, which also revealed a low percentage of chimeric mutants [53]. Since chimaeras are occasionally observed, thorough genotypic analysis will be required when phenotyping is performed in the $\mathrm{T}_{0}$ generation. Besides vegetative propagation, Parasponia trees can also be propagated generatively. Under suitable greenhouse conditions, Parasponia trees flower within 6-9 months and are self-compatible [4]. However, Parasponia trees can be monoecious or diecious and female flowers are wind pollinated [5]. This complicates selfing of trees and the production of pure seed badges. Additionally, Parasponia trees are fast growing and occupy a substantial amount of space in a tropical greenhouse $\left(28^{\circ} \mathrm{C}\right.$, $\sim 100 \%$ relative humidity), making generative propagation of multiple mutant lines logistically somewhat challenging. As an alternative for generative propagation, transgenic lines can be maintained in vitro and propagated vegetatively. Additionally, the fast and efficient transformation procedure presented here, will allow recreation of a particular mutant in less than six months.

Among the mutants we created, Panhk4 and Panein2 showed symbiotic phenotypes that differ from corresponding legume mutants. P. andersonii Panhk4 mutants form nodules with a wild-type cyto-architecture, suggesting that these nodules are fully functional. Analysis of stem cross-sections showed that Panhk4 mutants possess reduced cambial activity, suggesting that PanHK4 encodes a functional cytokinin receptor. $M$. truncatula and L. japonicus mutants in the cytokinin receptors orthologous to PanHK4 are characterized as nodulation deficient $[23,24]$. However, these mutants occasionally form nodules [24, $54,55]$. This suggests redundant functioning of additional cytokinin receptors in both legume species. The $P$. andersonii genome also encodes two additional cytokinin receptors: PanHK2 and PanHK3 [30] (Supplemental Figure 4). Therefore, redundant functioning of one of these receptors cannot be excluded. Additional experiments are required to examine this possibility and determine the exact contribution of cytokinin to Parasponia nodule formation. 
Mutation of PanEIN2 revealed a different function for ethylene signaling in regulation of rhizobium symbiosis between legumes and Parasponia. In legumes, ethylene negatively regulates rhizobial infection and root nodule formation $[27,28,36]$. This is illustrated by the phenotype of the $M$. truncatula ein2 mutant (named sickle) that forms extensive epidermal infection threads and clusters of small nodules [36, 56]. P. andersonii ein2 mutants also form smaller nodules than wild type. However, in contrast to the Mtein2 mutant, these nodules are regularly spaced on the root system. This suggests that in Parasponia ethylene signaling is not involved in regulating nodule number. Additionally, also the infection phenotype of Panein2 mutants differs from that in legumes. In M. truncatula and L. japonicus, interference with ethylene signaling increases the number of epidermal infection threads but does not affect intracellular colonization of nodule cells $[36,57,58]$. In contrast, in P. andersonii Panein2 mutants, intracellular colonization is hampered. Inside nodules, large apoplastic colonies are observed and fixation thread formation is severely reduced or even completely absent. This suggests that in Parasponia a functional ethylene signaling pathway is required for efficient intracellular infection of nodule cells. Since both nodule formation as well as intracellular infection are differentially regulated by ethylene in legumes and Parasponia, this suggests independent recruitment of ethylene function during evolution of rhizobium symbiosis in both lineages.

Mutagenesis of the NSP2 orthologue of $P$. andersonii indicated a conserved symbiotic role for this GRAS-type transcriptional regulator. In legumes, NSP2 works in concert with NSP1 to control root nodule formation [59]. Mutagenesis of the NSP1 orthologue of $P$. andersonii resulted in contrasting nodulation phenotypes. Two mutant lines, Pannsp1-6 and Pannsp1-13, form nodules with a wild-type cyto-architecture, whereas mutant line Pannsp1-39 is unable to form nodules (Figure 6-7). However, all three mutants are affected in transcriptional regulation of strigolactone biosynthesis genes PanD27 and PanMAX1 (Figure 5). The three Pannsp1 mutant lines differ from each other in the type of mutations that were created. Pannsp1-6 and Pannsp1-13 contain small deletions that are immediately followed by a second in-frame ATG that in wild-type PanNSP1 encodes a methionine at position 16. In contrast, Pannsp1-39 contains a larger deletion that removes this in-frame ATG (see Supplemental Figure 9). Several reports have shown that alternative start codons are occasionally used to initiate transcription [60-62]. Therefore, Pannsp1-6 and Pannsp1-13 most probably represent weak alleles that still possess residual PanNSP1 function. Such residual levels of PanNSP1 are affecting expression of strigolactone biosynthesis genes, but are still sufficient to allow nodule formation. Therefore, we argue that the $P$. andersonii Pannsp1-39 line carries a knockout mutation, indicating that in P. andersonii both NSP1 and NSP2 are essential for rhizobium root nodule formation. 
Taken together, we showed that $P$. andersonii can be efficiently transformed using $A$. tumefaciens and is amenable to targeted mutagenesis using CRISPR/Cas9. This protocol takes only marginally more time than the transient $A$. rhizogenes transformation system that is generally used to study root nodule formation [e.g. 18, 31, 63, 64, 65] but has several advantages. One of these is the absence of the A. rhizogenes root inducing locus (rol) that interferes with hormone homeostasis [66]. The protocol we developed will allow studies on $P$. andersonii symbiosis genes to determine to what extent legumes and Parasponia use similar mechanism to establish a nitrogen-fixing symbiosis with rhizobium. Additionally, Parasponia might present an excellent reverse genetics platform to ask basic questions in relation to general tree biology.

\section{Methods}

\section{Plant Materials and Growth Conditions}

All experiments were conducted using Parasponia andersonii WU1 or offspring thereof [18, 30]. $P$. andersonii trees were grown in a conditioned greenhouse at $28^{\circ} \mathrm{C}, 85 \%$ humidity and a $16 / 8$ h day/night regime. For in vitro culturing, P. andersonii was grown in an Elbanton growth cabinet at $28^{\circ} \mathrm{C}, 16 / 8 \mathrm{~h}$ day/night. Growth of young $P$. andersonii plantlets for nodulation assays or qRT-PCR analysis was performed in $1 \mathrm{~L}$ crystal-clear polypropelene containers equipped with an gas exchange filter (OS140BOX, Duchefa Biochemie, The Netherlands). Pots were half-filled with agraperlite (Maasmond-Westland, The Netherlands) and watered with modified EKM medium ( $3 \mathrm{mM} \mathrm{MES}\left(\mathrm{C}_{6} \mathrm{H}_{13} \mathrm{NO}_{4}\right) \mathrm{pH} 6.6,2.08 \mathrm{mM} \mathrm{MgSO}, 0.88 \mathrm{mM}$ $\mathrm{KH}_{2} \mathrm{PO}_{4}, 2.07 \mathrm{mM} \mathrm{K}_{2} \mathrm{HPO}_{4}, 1.45 \mathrm{mM} \mathrm{CaCl}_{2}, 0.70 \mathrm{mM} \mathrm{Na}_{2} \mathrm{SO}_{4}, 0.375 \mathrm{mM} \mathrm{NH}_{4} \mathrm{NO}_{3}, 15 \mu \mathrm{M} \mathrm{Fe}-$ citrate, $6.6 \mu \mathrm{M} \mathrm{MnSO}_{4}, 1.5 \mu \mathrm{M} \mathrm{ZnSO}_{4}, 1.6 \mu \mathrm{M} \mathrm{CuSO}_{4}, 4 \mu \mathrm{M} \mathrm{H}_{3} \mathrm{BO}_{3}, 4.1 \mu \mathrm{M} \mathrm{Na}_{2} \mathrm{MoO}_{4}$ ) [67] and placed in a climate room set at $28^{\circ} \mathrm{C}, 16 / 8 \mathrm{~h}$ day/night. For nodulation assays, EKM medium was inoculated with Mesorhizobium plurifarium BOR2 $\left(\mathrm{OD}_{600}=0.025\right)[30]$.

\section{Vectors and Constructs}

For CRISPR/Cas9-mediated mutagenesis, binary transformation constructs were created using Golden Gate assembly [68]. For an overview of all Golden Gate clones used in this study, see Supplemental Table 5. sgRNAs were designed based on the principles described in Doench et al. [69] and PCR amplified using specific forward primers and a universal reverse primer (Supplemental Table 6), using Addgene plasmid \# 46966 as template [38]. These were cloned behind the AtU6p small RNA promoter and inserted behind the neomycin phosphotransferease II gene (NPTII) and an A. thaliana codon-optimized variant of Cas9 [40] fused to an N-terminal nuclear localization signal and driven by the $35 \mathrm{~S}$ promoter 
(Supplemental Table 5). As negative control, a binary vector was created containing only the NPTII- and NLS-Cas9-encoding sequences (Supplemental Table 5). To setup P. andersonii stable transformation, vector pKGWFS7-RR was used [70].

\section{Phylogenetic Reconstruction}

Protein sequences of Glycine max (Wm82.a2.v1) [71], Medicago truncatula (Mt4.0v1) [72, 73] and Populus trichocarpa (v3.0) [74] were obtained through Phytozome 10 (http:// phytozome.jgi.doe.gov/). Protein sequences of Parasponia andersonii (PanWU01x14_ asm01_ann01) and Trema orientalis (TorRG33x02_asm01_ann01) were obtained from www.parasponia.org [30]. These sequences were mined using sequences from Arabidopsis thaliana (TAIR10, www.arabidopsis.org) [75] and M. truncatula. Protein sequences were aligned using MAFFT v7.017 [76] implemented in Geneious 8.1.9 (Biomatters, New Zealand), using default parameter settings. Phylogenetic trees were constructed using FastTree [77] implemented in Geneious 8.1.9. Mid-point rooting was applied for better tree visualization using FigTree v1.4.2 (http://tree.bio.ed.ac.uk/software/figtree/).

\section{Plant Transformation}

Stable transformation of $P$. andersonii was performed using Agrobacterium tumefaciens strain AGL1 [78]. A. tumefaciens was grown for two days on agar-solidified LB medium containing appropriate antibiotics. For each $P$. andersonii transformation, two Petri dishes $(\varnothing 9 \mathrm{~cm})$ of $A$. tumefaciens were used. Bacteria were scraped from plate and resuspended in $25 \mathrm{ml}$ of infiltration medium (SH10 (Supplemental Table 7), $20 \mathrm{mg} / \mathrm{l}$ acetosyringone (Sigma, USA), 0.001\% (v/v) Silwet L-77 (www.arabidopsis.com)). P. andersonii tissue explants used for transformation were harvested from mature trees grown under greenhouse conditions and sterilized in $2 \%$ commercial bleach for 15 minutes. Tissue explants were cut at both ends inside the $A$. tumefaciens suspension, creating fresh wound surfaces, and kept inside the suspension for about 20 minutes. Subsequently, excess liquid was removed from tissue explants using sterilized filter paper and explants were placed on co-cultivation medium (Root-inducing medium (Supplemental Table 7), $20 \mathrm{mg} / \mathrm{l}$ acetosyringone (Sigma, USA)). Plates were incubated for two days at $21^{\circ} \mathrm{C}$ in darkness. After two days, tissue explants were washed three times using $\mathrm{SH} 10$ (Supplemental Table 7) and subsequently dried using filter paper. Tissue explants were placed on root-inducing medium containing $50 \mathrm{mg} / \mathrm{l}$ kanamycin and $300 \mathrm{mg} / \mathrm{l}$ cefotaxime and incubated at $28^{\circ} \mathrm{C}, 16 \mathrm{~h} / 8 \mathrm{~h}$ (day/night). Nine days after transformation, tissue explants were transferred to propagation medium (Supplemental Table 7) containing $50 \mathrm{mg} / \mathrm{l}$ kanamycin and $300 \mathrm{mg} / \mathrm{l}$ cefotaxime. Plates were refreshed every other week. When regenerative calli reached $\sim 2 \mathrm{~mm}$ in size they were separated 
from tissue explants to stimulate shoot formation. A single shoot was selected per tissue explant. These shoots were propagated on propagation medium (Supplemental Table 7). Rooted plantlets were generated by placing individual shoots on root-inducing medium (Supplemental Table 7).

\section{Characterization of Transgenic Lines}

For T-DNA copy number estimates based on qPCR analysis, genomic DNA was isolated using the DNeasy Plant Mini Kit (Qiagen, Germany). qPCR was set up in a $10 \mu$ reaction system with 2x iQ SYBR Green Super-mix (Bio-Rad, Hercules, USA) and 5 ng template DNA. The experimental setup and procedure were executed on a CFX Connect optical cycler, according to the manufacturer's protocol (Bio-Rad, USA). T-DNA copy number was estimated using two primer pairs amplifying part of the T-DNA and two primer pairs amplifying single copy $P$. andersonii genes (PanAGT1 and PanWU01x14_asm01_ann01_338920) that were selected based on a study by Duarte et al. [79]. Primer sequences are listed in Supplemental Table 6. Data analysis was performed using CFX Manager 3.0 software (Bio-Rad, USA). For T-DNA copy number estimates based on Southern blotting, genomic DNA was separately digested with Xbal, HindIII and EcoRI. Blots were hybridized with a 516 bp $\alpha$-32P-labelled probe corresponding to part of the $n p t / l$ gene that was amplified using primers nptll_Fw and nptII_ Rv listed in Supplemental Table 6.

Genotyping of transgenic lines was performed using the Phire Plant Direct PCR Kit (Thermo Scientific, USA) and gene specific primers listed in Supplemental Table 6. Ploidy estimates of transgenic lines were determined by FACS as described by van Velzen et al. [30].

To determine ethylene sensitivity of Panein2 mutants, tips of young branches of four months-old trees were covered with $1 \mathrm{~L}$ plastic bags and injected with $1 \mathrm{ml}$ of pure ethylene gas. After three days, bags were removed and leaf abscission examined. Total number of leaves on treated branches varied from 6-18.

\section{Microtome Sectioning}

Stem cross-sections were made from the base of the second uppermost axillary branch of three month-old trees. Shoot tissue was fixed in $5 \%$ glutaraldehyde and embedded in Technovit 7100 (Heraeus-Kulzer, Germany), according to the manufacturer's protocol. Semithin $(8 \mu \mathrm{m})$ sections were cut using a microtome (Reichert-Jung, Leica Microsystems, The Netherlands) and stained with $0.05 \%$ Toluidine Blue O. Images were taken using a Leica DM5500B microscope equipped with a DFC425C camera (Leica Microsystems, Germany). 
Nodule tissue fixation and embedding was performed as previously described [80]. Semithin $(0.6 \mu \mathrm{m})$ sections were cut using a Leica Ultracut microtome (Leica Microsystems, Germany) and photographed as described above.

\section{RNA Isolation and qRT-PCR Analysis}

RNA was isolated from snap-frozen root tips $(\sim 2-3 \mathrm{~cm})$ as described by van Velzen et al. [30]. cDNA was prepared from $1 \mu \mathrm{g}$ of total RNA using the i-script cDNA synthesis kit (Bio-Rad, USA), following the manufacturer's instructions. RT-qPCR was set up as described above. Normalization was performed based on two stably expressed reference genes (PanUNK2, PanEF1 $\alpha)$, chosen based on previous study [81, 82]. All primers are listed in Supplemental Table 6.

\section{Statistical Analysis}

Statistical differences were determined based on One-way ANOVA and Tukey post-hoc tests. Statistical analyses were performed using IBM SPSS Statistics 23.0 (IBM, USA).

\section{Accession Numbers}

Gene identifiers for all $P$. andersonii genes used in this study can be found in Supplemental Table 8. Sequences can be downloaded from www.parasponia.org

\section{Author Contributions}

Conceptualization, A.v.Z. and R.G.; Methodology, A.v.Z., M.H., S.L. and WK; Investigation, A.v.Z., T.A.K.W., M.S.K., L.R., F.B., M.H., S.L., E.F. and W.K.; Formal Analysis, A.v.Z., T.A.K.W. and E.F.; Visualization, A.v.Z.; Writing - Original Draft, A.v.Z.; Writing - Review \& Editing, A.v.Z. and R.G.; Funding Acquisition, T.B. and R.G.; Supervision, R.G.

\section{ACKNOWLEDGEMENTS}

The authors like to thank Renze Heidstra for help with FACS analysis and Michiel Lammers and Renze Heidstra for useful tips regarding CRISPR/Cas9 strategy. Ethylene gas was kindly provided by Arjen van de Peppel and Julian Verdonk. Golden Gate parts and cloning vectors were kindly provided by Mark Youles, Sophien Kamoun and Sylvestre Marillonnet through the Addgene database. This work was supported by NWO-NSFC Joint Research project (846.11.005) to T.B. and R.G., NWO-VICI (865.13.001) to R.G., NWO-VENI (863.15.010) to W.K., European Research Council (ERC-2011-AdG294790) to T.B. and China Scholarship Councils (201303250067) to F.B. 


\section{References}

1 Akkermans, A.D.L. et al. (1978) Nitrogen-fixing root nodules in Ulmaceae. Nature 274, 190

2 Clason, E.W. (1936) The vegetation of the upper-Badak region of mount Kelut (east java). Bulletin Jard. Bot. Buitenzorg Serie III, 509-518

3 Trinick, M.J. (1973) Symbiosis between Rhizobium and the non-legume, Trema aspera. Nature 244, 459-460

4 Becking, J.H. (1992) The Rhizobium symbiosis of the nonlegume Parasponia. In Biological nitrogen fixation, pp. 497-559, Routledge, Chapman and Hall

5 Soepadmo, E. (1974) Ulmaceae. Flora Malesiana-Series 1, Spermatophyta 8, 31-76

6 Trinick, M.J. (1980) Growth of Parasponia in agar tube culture and symbiotic effectiveness of isolates from Parasponia spp. New Phytol. 85, 37-45

7 Trinick, M.J. (1981) The effective rhizobium symbiosis with the non-legume Parasponia andersonii. In Current Perspectives in Nitrogen Fixation (Gibson, A.H. and Newton, W.E., eds), pp. 480, Australian Academy of Sciences.

8 Trinick, M.J. and Hadobas, P.A. (1989) Biology of the Parasponia-Bradyrhizobium symbiosis. In Nitrogen Fixation with Non-Legumes, pp. 25-33, Springer Netherlands

9 Behm, J.E. et al. (2014) Parasponia: a novel system for studying mutualism stability. Trends Plant Sci. 19, 757763

10 Geurts, R. et al. (2012) Exploiting an ancient signalling machinery to enjoy a nitrogen fixing symbiosis. Curr. Opin. Plant Biol. 15, 438-443

11 Geurts, R. et al. (2016) What does it take to evolve a nitrogen-fixing endosymbiosis? Trends Plant Sci. 21, 199-208

12 Jansson, S. and Douglas, C.J. (2007) Populus: a model system for plant biology. Annu. Rev. Plant Biol. 58, 435458

13 Neale, D.B. et al. (2017) Novel insights into tree biology and genome evolution as revealed through genomics. Annu. Rev. Plant Biol. 68, 457-483

14 Wang, H. et al. (2009) Rosid radiation and the rapid rise of angiosperm-dominated forests. Proc. Natl. Acad. Sci. U. S. A. 106, 3853-3858

15 Li, H.-L. et al. (2015) Large-scale phylogenetic analyses reveal multiple gains of actinorhizal nitrogen-fixing symbioses in angiosperms associated with climate change. Sci. Rep. 5, 14023

16 Granqvist, E. et al. (2015) Bacterial-induced calcium oscillations are common to nitrogen-fixing associations of nodulating legumes and non-legumes. New Phytol. 207, 551-558

17 Marvel, D.J. et al. (1987) Rhizobium symbiotic genes required for nodulation of legume and nonlegume hosts. Proc. Natl. Acad. Sci. U. S. A. 84, 1319-1323

18 Op den Camp, R. et al. (2011) LysM-type mycorrhizal receptor recruited for rhizobium symbiosis in nonlegume Parasponia. Science 331, 909-912

19 Oldroyd, G.E.D. (2013) Speak, friend, and enter: signalling systems that promote beneficial symbiotic associations in plants. Nat. Rev. Microbiol. 11, 252-263

20 Gonzalez-Rizzo, S. et al. (2006) The Medicago truncatula CRE1 cytokinin receptor regulates lateral root development and early symbiotic interaction with Sinorhizobium meliloti. Plant Cell 18, 2680-2693

21 Heckmann, A.B. et al. (2006) Lotus japonicus nodulation requires two GRAS domain regulators, one of which is functionally conserved in a non-legume. Plant Physiol. 142, 1739-1750

22 Kaló, P. et al. (2005) Nodulation signaling in legumes requires NSP2, a member of the GRAS family of transcriptional regulators. Science 308, 1786-1789

23 Murray, J.D. et al. (2007) A cytokinin perception mutant colonized by Rhizobium in the absence of nodule organogenesis. Science 315, 101-104

24 Plet, J. et al. (2011) MtCRE1-dependent cytokinin signaling integrates bacterial and plant cues to coordinate symbiotic nodule organogenesis in Medicago truncatula. Plant J. 65, 622-633

25 Smit, P. et al. (2005) NSP1 of the GRAS protein family is essential for rhizobial Nod factor-induced transcription. Science 308, 1789-1791

26 Tirichine, L. et al. (2007) A gain-of-function mutation in a cytokinin receptor triggers spontaneous root nodule organogenesis. Science 315, 104-107 
27 Miyata, K. et al. (2013) Two distinct EIN2 genes cooperatively regulate ethylene signaling in Lotus japonicus. Plant Cell Physiol. 54, 1469-1477

28 Penmetsa, V.R. et al. (2008) The Medicago truncatula ortholog of Arabidopsis EIN2, sickle, is a negative regulator of symbiotic and pathogenic microbial associations. Plant J. 55, 580-595

29 van Zeijl, A. et al. (2015) Rhizobium lipo-chitooligosaccharide signaling triggers accumulation of cytokinins in Medicago truncatula roots. Mol. Plant 8, 1213-1226

30 van Velzen, R. et al. (2017) Parallel loss of symbiosis genes in relatives of nitrogen-fixing non-legume Parasponia. bioRxiv DOI: $10.1101 / 169706$

31 Cao, Q. et al. (2012) Efficiency of Agrobacterium rhizogenes-mediated root transformation of Parasponia and Trema is temperature dependent. Plant Growth Regul. 68, 459-465

32 Davey, M.R. et al. (1993) Effective nodulation of micro-propagated shoots of the non-legume Parasponia andersonii by Bradyrhizobium. J. Exp. Bot. 44, 863-867

33 Webster, G. et al. (1995) The nodulation of micro-propagated plants of Parasponia andersonii by tropical legume rhizobia. J. Exp. Bot. 46, 1131-1137

34 Liu, W. et al. (2011) Strigolactone biosynthesis in Medicago truncatula and rice requires the symbiotic GRAStype transcription factors NSP1 and NSP2. Plant Cell 23, 3853-3865

35 van Zeijl, A. et al. (2015) The strigolactone biosynthesis gene DWARF27 is co-opted in rhizobium symbiosis. BMC Plant Biol. 15, 260

36 Penmetsa, R.V. and Cook, D.R. (1997) A legume ethylene-insensitive mutant hyperinfected by its rhizobial symbiont. Science 275, 527-530

37 Kareem, A. et al. (2015) PLETHORA genes control regeneration by a two-step mechanism. Curr. Biol. 25, 10171030

38 Nekrasov, V. et al. (2013) Targeted mutagenesis in the model plant Nicotiana benthamiana using Cas9 RNAguided endonuclease. Nat. Biotechnol. 31, 691-693

39 Engler, C. et al. (2014) A Golden Gate modular cloning toolbox for plants. ACS Synth. Biol. 3, 839-843

40 Fauser, F. et al. (2014) Both CRISPR/Cas-based nucleases and nickases can be used efficiently for genome engineering in Arabidopsis thaliana. Plant J. 79, 348-359

41 Agustí, J. et al. (2009) Comparative transcriptional survey between laser-microdissected cells from laminar abscission zone and petiolar cortical tissue during ethylene-promoted abscission in citrus leaves. BMC Plant Biol. 9, 127

42 Brown, K.M. (1997) Ethylene and abscission. Physiol. Plant. 100, 567-576

43 Jackson, M.B. and Osborne, D.J. (1970) Ethylene, the natural regulator of leaf abscission. Nature 225, 10191022

44 Bhalerao, R.P. and Fischer, U. (2017) Environmental and hormonal control of cambial stem cell dynamics. J. Exp. Bot. 68, 79-87

45 Matsumoto-Kitano, M. et al. (2008) Cytokinins are central regulators of cambial activity. Proc. Natl. Acad. Sci. U. S. A. $105,20027-20031$

46 Nieminen, K. et al. (2008) Cytokinin signaling regulates cambial development in poplar. Proc. Natl. Acad. Sci. U. S. A. $105,20032-20037$

47 Cardoso, C. et al. (2014) Natural variation of rice strigolactone biosynthesis is associated with the deletion of two MAX1 orthologs. Proc. Natl. Acad. Sci. U. S. A. 111, 2379-2384

48 Zhang, Y.X. et al. (2014) Rice cytochrome P450 MAX1 homologs catalyze distinct steps in strigolactone biosynthesis. Nat. Chem. Biol. 10, 1028-1033

49 Shtark, O.Y. et al. (2016) Arbuscular mycorrhiza development in pea (Pisum sativum L.) mutants impaired in five early nodulation genes including putative orthologs of NSP1 and NSP2. Symbiosis 68, 129-144

50 Op den Camp, R.H.M. et al. (2012) Nonlegume Parasponia andersonii deploys a broad Rhizobium host range strategy resulting in largely variable symbiotic effectiveness. Mol. Plant. Microbe Interact. 25, 954-963

51 Altpeter, F. et al. (2016) Advancing crop transformation in the era of genome editing. Plant Cell 28, 1510-1520

52 Ledford, H. (2016) A better way to hack plant DNA. Nature 539, 16-17

53 Fan, D. et al. (2015) Efficient CRISPR/Cas9-mediated targeted mutagenesis in Populus in the first generation. Sci. Rep. 5, 12217

54 Held, M. et al. (2014) Lotus japonicus cytokinin receptors work partially redundantly to mediate nodule formation. Plant Cell 26, 678-694 
55 Boivin, S. et al. (2016) Different cytokinin histidine kinase receptors regulate nodule initiation as well as later nodule developmental stages in Medicago truncatula. Plant Cell Environ. 39, 2198-2209

56 Xiao, T.T. et al. (2014) Fate map of Medicago truncatula root nodules. Development 141, 3517-3528

57 Lohar, D. et al. (2009) Ethylene insensitivity conferred by a mutated Arabidopsis ethylene receptor gene alters nodulation in transgenic Lotus japonicus. Ann. Bot. 104, 277-285

58 Nukui, N. et al. (2004) Transgenic Lotus japonicus with an ethylene receptor gene Cm-ERS1/H7OA enhances formation of infection threads and nodule primordia. Plant Cell Physiol. 45, 427-435

59 Hirsch, S. et al. (2009) GRAS proteins form a DNA binding complex to induce gene expression during nodulation signaling in Medicago truncatula. Plant Cell 21, 545-557

60 Bazykin, G.A. and Kochetov, A.V. (2011) Alternative translation start sites are conserved in eukaryotic genomes. Nucleic Acids Res. 39, 567-577

61 Chabregas, S.M. et al. (2003) Differential usage of two in-frame translational start codons regulates subcellular localization of Arabidopsis thaliana THI1. J. Cell Sci. 116, 285-291

62 Thatcher, L.F. et al. (2007) Differential gene expression and subcellular targeting of Arabidopsis glutathione S-transferase F8 is achieved through alternative transcription start sites. J. Biol. Chem. 282, 28915-28928

63 Boisson-Dernier, A. et al. (2001) Agrobacterium rhizogenes-transformed roots of Medicago truncatula for the study of nitrogen-fixing and endomycorrhizal symbiotic associations. Mol. Plant. Microbe Interact. 14, 695-700

64 Kumagai, H. and Kouchi, H. (2003) Gene silencing by expression of hairpin RNA in Lotus japonicus roots and root nodules. Mol. Plant. Microbe Interact. 16, 663-668

65 Limpens, E. et al. (2004) RNA interference in Agrobacterium rhizogenes-transformed roots of Arabidopsis and Medicago truncatula. J. Exp. Bot. 55, 983-992

66 Nilsson, O. and Olsson, O. (1997) Getting to the root: The role of the Agrobacterium rhizogenes rol genes in the formation of hairy roots. Physiol. Plant. 100, 463-473

67 Becking, J.H. (1983) The Parasponia parviflora - rhizobium symbiosis - isotopic nitrogen-fixation, hydrogen evolution and nitrogen-fixation efficiency, and oxygen relations. Plant Soil 75, 343-360

68 Engler, C. et al. (2009) Golden Gate shuffling: a one-pot DNA shuffling method based on Type Ils restriction enzymes. PLoS One 4, e5553

69 Doench, J.G. et al. (2014) Rational design of highly active sgRNAs for CRISPR-Cas9-mediated gene inactivation. Nat. Biotechnol. 32, 1262-1267

70 Karimi, M. et al. (2002) GATEWAY ${ }^{\mathrm{TM}}$ vectors for Agrobacterium-mediated plant transformation. Trends Plant Sci. 7, 193-195

71 Schmutz, J. et al. (2010) Genome sequence of the palaeopolyploid soybean. Nature 463, 178-183

72 Young, N.D. et al. (2011) The Medicago genome provides insight into the evolution of rhizobial symbioses. Nature 480, 520-524

73 Tang, H.B. et al. (2014) An improved genome release (version Mt4.0) for the model legume Medicago truncatula. BMC Genomics 15, 312

74 Tuskan, G.A. et al. (2006) The genome of black cottonwood, Populus trichocarpa (Torr. \& Gray). Science 313, 1596-1604

75 Lamesch, P. et al. (2012) The Arabidopsis Information Resource (TAIR): improved gene annotation and new tools. Nucleic Acids Res. 40, D1202-D1210

76 Katoh, K. et al. (2002) MAFFT: a novel method for rapid multiple sequence alignment based on fast Fourier transform. Nucleic Acids Res. 30, 3059-3066

77 Price, M.N. et al. (2009) FastTree: computing large minimum evolution trees with profiles instead of a distance matrix. Mol. Biol. Evol. 26, 1641-1650

78 Lazo, G.R. et al. (1991) A DNA transformation-competent Arabidopsis genomic library in Agrobacterium. Biotechnology 9, 963-967

79 Duarte, J.M. et al. (2010) Identification of shared single copy nuclear genes in Arabidopsis, Populus, Vitis and Oryza and their phylogenetic utility across various taxonomic levels. BMC Evol. Biol. 10, 61

80 Fedorova, E. et al. (1999) Localization of $\mathrm{H}^{+}$-ATPase in soybean root nodules. Planta 209, 25-32

81 Bansal, R. et al. (2015) Recommended reference genes for quantitative PCR analysis in soybean have variable stabilities during diverse biotic stresses. PLoS One 10, e0134890

82 Czechowski, T. et al. (2005) Genome-wide identification and testing of superior reference genes for transcript normalization in Arabidopsis. Plant Physiol. 139, 5-17 


\section{SUPPLEMENTAL DATA}

Supplemental Table 1. Regeneration efficiency of different explant types. Regeneration was determined for non-transgenic material incubated on propagation medium.

\begin{tabular}{cc}
\hline Explant type & Regeneration efficiency \\
\hline Young leaves & - \\
Mature leaves & $+/-$ \\
Midveins & $+/-$ \\
Stem pieces $(5 \mathrm{~mm})$ & ++ \\
Stem pieces $(1 \mathrm{~mm})$ & $+/-$ \\
Stem pieces, cut length-wise & + \\
Petioles & +++ \\
Shoot apical meristems & - \\
Tissue culture shoots & + \\
\hline
\end{tabular}

+++ means that $>90 \%$ of explants develop multiple regenerative calli.

-, indicates that no regeneration was observed, tissue turned dark brown in color.

Supplemental Table 2. Hormone composition of tissue culture media used in this study.

\begin{tabular}{|c|c|c|c|c|c|c|c|c|c|c|c|}
\hline \multirow{2}{*}{ Hormone } & \multirow{2}{*}{$\begin{array}{l}\text { Propagation } \\
\text { medium }\end{array}$} & \multirow{2}{*}{$\begin{array}{c}\text { Root- } \\
\text { inducing } \\
\text { medium }\end{array}$} & \multicolumn{9}{|c|}{ Alternative media } \\
\hline & & & 1 & 2 & 3 & 4 & 5 & 6 & 7 & 8 & 9 \\
\hline $\mathrm{BAP}(\mathrm{mg} / \mathrm{L})$ & 1 & - & 0.33 & 1 & 3 & 0.33 & 1 & 3 & 0.33 & 1 & 3 \\
\hline IBA (mg/L) & 0.1 & 1 & - & - & - & - & - & - & - & - & - \\
\hline $\mathrm{NAA}(\mathrm{mg} / \mathrm{L})$ & - & 0.1 & 0.03 & 0.03 & 0.03 & 0.1 & 0.1 & 0.1 & 0.3 & 0.3 & 0.3 \\
\hline
\end{tabular}

Supplemental Table 3. Regeneration of transgenic material after co-cultivation and continuous culturing on propagation medium (Prop) or co-cultivation and culturing for seven additional days on root-inducing medium and subsequent transfer to propagation medium (Root -> prop). Regeneration was scored 6 weeks after start of transformation.

\begin{tabular}{ccc}
\hline & Prop & Root $->$ prop \\
\hline Petioles & + & ++ \\
Stem pieces $(5 \mathrm{~mm})$ & $+/-$ & ++ \\
\hline
\end{tabular}

++ , means that $>50 \%$ of explants develop regenerative calli.

- , means that $<20 \%$ of explants develop regenerative calli. 
Supplemental Table 4. Molecular characterization of transgenic lines.

\begin{tabular}{|c|c|c|c|c|}
\hline \multirow{2}{*}{ Line $^{1}$} & \multicolumn{2}{|c|}{ T-DNA copy number estimate } & \multirow{2}{*}{ Ploidy } & \multirow{2}{*}{$\begin{array}{l}\text { Presence of } \\
\text { right border } \\
\text { sequences }^{4}\end{array}$} \\
\hline & qRT-PCR ${ }^{2}$ & Southern blot ${ }^{3}$ & & \\
\hline 1 & 2.9 & na & Diploid & + \\
\hline $3^{*}$ & 1.0 & 1 & Diploid & + \\
\hline 4 & 1.2 & na & Diploid & + \\
\hline 12 & 0.9 & na & Diploid & + \\
\hline 13 & 1.0 & 2 & Diploid & + \\
\hline 18 & 1.9 & na & Diploid & + \\
\hline 19 & 2.9 & 3 & Diploid & + \\
\hline 21 & 1.1 & na & Diploid & + \\
\hline 31 & 1.9 & 2 & Diploid & + \\
\hline $33^{*}$ & 1.0 & 1 & Diploid & + \\
\hline 37 & 2.1 & na & Diploid & + \\
\hline $43^{*}$ & 0.9 & 1 & Diploid & + \\
\hline 47 & 2.8 & na & Diploid & + \\
\hline 49 & 1.1 & na & Diploid & + \\
\hline 50 & nd & na & Diploid & - \\
\hline 52 & 1.0 & 1 & Diploid & + \\
\hline 54 & 0.5 & 2 & Diploid & + \\
\hline 55 & 0.8 & na & Diploid & + \\
\hline 59 & 0.4 & na & Diploid & + \\
\hline 61 & 0.9 & na & Diploid & + \\
\hline
\end{tabular}


Supplemental Table 5. List of Golden Gate ${ }^{1}$ constructs used in this study.

\begin{tabular}{|c|c|c|c|c|c|}
\hline Construct & Description & Level & Backbone & Contains $^{2}$ & Literature \\
\hline 1 & npt/l resistance cassette & 1 & $\mathrm{plCH} 47802$ & pICSL70004:nptII & - \\
\hline 2 & $35 \mathrm{~S}_{\text {pro }}:$ NNLS-Cas9:35S & 1 & $\mathrm{plCH} 47742$ & 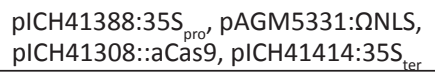 & $\begin{array}{l}\text { Fauser et } \\
\text { al. [42] }\end{array}$ \\
\hline 3 & PanEIN2sgRNA1 & 1 & plCH47751 & $\begin{array}{l}\text { pICSL01009:AtU6p, corresponding } \\
\text { PCR amplicon }\end{array}$ & $\begin{array}{l}\text { Nekrasov } \\
\text { et al. [40] }\end{array}$ \\
\hline 4 & PanNSP1sgRNA1 & 1 & plCH47751 & $\begin{array}{l}\text { pICSL01009:AtU6p, corresponding } \\
\text { PCR amplicon }\end{array}$ & $\begin{array}{l}\text { Nekrasov } \\
\text { et al. [40] }\end{array}$ \\
\hline 5 & PanNSP2sgRNA1 & 1 & plCH47751 & $\begin{array}{l}\text { pICSL01009:AtU6p, corresponding } \\
\text { PCR amplicon }\end{array}$ & $\begin{array}{l}\text { Nekrasov } \\
\text { et al. [40] }\end{array}$ \\
\hline 6 & PanNSP2sgRNA2 & 1 & plCH47761 & $\begin{array}{l}\text { pICSL01009:AtU6p, corresponding } \\
\text { PCR amplicon }\end{array}$ & $\begin{array}{l}\text { Nekrasov } \\
\text { et al. [40] }\end{array}$ \\
\hline 7 & PanNSP2sgRNA3 & 1 & $\mathrm{plCH} 47841$ & $\begin{array}{l}\text { pICSL01009:AtU6p, corresponding } \\
\text { PCR amplicon }\end{array}$ & $\begin{array}{l}\text { Nekrasov } \\
\text { et al. [40] }\end{array}$ \\
\hline 8 & CRISPR_ctrl & 2 & pICSL4723 & $\begin{array}{l}\text { 1R: construct 1, 2F: construct 2, end- } \\
\text { link pICH41744 }\end{array}$ & - \\
\hline 9 & CRISPR_PanEIN2 & 2 & pICSL4723 & $\begin{array}{l}\text { 1R: construct } 1,2 \mathrm{~F} \text { : construct 2, 3F: } \\
\text { construct } 3 \text {, end-link pICH41766 }\end{array}$ & - \\
\hline 10 & CRISPR_PanNSP1 & 2 & pICSL4723 & $\begin{array}{l}\text { 1R: construct 1, 2F: construct 2, 3F: } \\
\text { construct 4, end-link pICH41766 }\end{array}$ & - \\
\hline 11 & CRISPR_PanNSP2 & 2 & pICSL4723 & $\begin{array}{l}\text { 1R: construct } 1,2 \mathrm{~F} \text { : construct } 2,3 \mathrm{~F} \text { : } \\
\text { construct } 5,4 \mathrm{~F} \text { : construct } 6,5 \mathrm{R} \text { : } \\
\text { construct } 7 \text {, end-link pICH41800 }\end{array}$ & - \\
\hline
\end{tabular}


Supplemental Table 6. Primers used in this study.

\begin{tabular}{|c|c|c|}
\hline Name & Purpose & Sequence \\
\hline PanEIN2_sgRNA1 & Clone sgRNA & $\begin{array}{l}\text { tgtggtctcaattGTGTTAATCCTGGA } \\
\text { AAATGGGgttttagagctagaaatagcaag }\end{array}$ \\
\hline PanNSP1_sgRNA1 & Clone sgRNA & $\begin{array}{l}\text { tgtggtctcaattGCCAGTCCATGATG } \\
\text { TGATCCGgttttagagctagaaatagcaag }\end{array}$ \\
\hline PanNSP2_sgRNA1 & Clone sgRNA & $\begin{array}{l}\text { tgtggtctcaattGAAGACGACCATGG } \\
\text { CTGCGCGgttttagagctagaaatagcaag }\end{array}$ \\
\hline PanNSP2_sgRNA2 & Clone sgRNA & $\begin{array}{l}\text { tgtggtctcaattGACTGGAACGTCC } \\
\text { TTACCGGgttttagagctagaaatagcaag }\end{array}$ \\
\hline PanNSP2_sgRNA3 & Clone sgRNA & $\begin{array}{l}\text { tgtggtctcaattGTAGTTCGACACCG } \\
\text { CCTACGGgttttagagctagaaatagcaag }\end{array}$ \\
\hline sgRNA_Rv & Clone sgRNA & tgtggtctcaAGCGTAATGCCAACTTTGTAC \\
\hline geno_Cas9_Fw & Amplify aCas9 & TTCGATCTCGCTGAGGATGC \\
\hline geno_Cas9_Rv & Amplify aCas9 & TAGCGAGAGGTCCCACGTAG \\
\hline geno_PanEIN2-crispr-Fw & Genotyping CRISPR mutants & CATTGCAACAGCCTGTGGAC \\
\hline geno_PanEIN2-crispr-Rv & Genotyping CRISPR mutants & CTGAGCAAGACCCCTTCCAG \\
\hline geno_PanNSP1-crispr-Fw & Genotyping CRISPR mutants & TGGTTTCTCGTGGCCTTTGT \\
\hline geno_PanNSP1-crispr-Rv & Genotyping CRISPR mutants & CTGTGGCCTTAGCTGAGCTT \\
\hline geno_PanNSP2-crispr-Fw & Genotyping CRISPR mutants & АCTTCCACTCTGTCCCCGAA \\
\hline geno_PanNSP2-crispr-Rv & Genotyping CRISPR mutants & GACCGGTGACTGAAGTGAGG \\
\hline qPanD27_Fw & qRT-PCR & TCGGATCGCCATTCAACATC \\
\hline qPanD27_Rv & qRT-PCR & GACAAGTTCCCGCTGTTTTG \\
\hline qPanMAX1_Fw & qRT-PCR & TGATGAGATCGTGGCCAAGAG \\
\hline qPanMAX1_Rv & qRT-PCR & AAAAACGTTCCTCGCAACCG \\
\hline qPanUNK2_Fw & qRT-PCR & TGCCATTGGTGTTAGCTGTG \\
\hline qPanUNK2_Rv & qRT-PCR & GTGTCTATCACTGCCTCTTTGC \\
\hline qPanEF1 $\alpha \_F w$ & qRT-PCR & AGACAAGGTTAAGCGTGCAG \\
\hline qPanEF1a_Rv & qRT-PCR & TGCAACTGGGCAACAAACTC \\
\hline qTCN-1_Fw & T-DNA copy number & AAGCGCGTTACAAGAAAGCC \\
\hline qTCN-1_Rv & T-DNA copy number & ACGTTGCCCGCATAATTACG \\
\hline qTCN-3_Fw & T-DNA copy number & TGCACGACCACGCATTAATG \\
\hline qTCN-3_Rv & T-DNA copy number & ACGATGCCATGTTCATCTGC \\
\hline PanAGT1_Fw & T-DNA copy number & AGCGCTTGATCTCCTTTTCG \\
\hline PanAGT1_Rv & T-DNA copy number & TGCTTTACCTTGTGGCAGTC \\
\hline Pan338920_Fw & T-DNA copy number & TGAAGCACAACACACGATGG \\
\hline Pan338920_Rv & T-DNA copy number & TGCTTTCAGCCAGTTAACCG \\
\hline nptll_Fw & Southern blotting & CACAACAGACAATCGGCTGC \\
\hline nptll_Rv & Southern blotting & TGATATTCGGCAAGCAGGCA \\
\hline
\end{tabular}


Supplemental Table 7. Composition of tissue culture media.

\begin{tabular}{|c|c|c|c|}
\hline \multirow{2}{*}{ Component } & \multicolumn{3}{|c|}{ Medium } \\
\hline & SH10 & Propagation & Root-inducing \\
\hline Schenk \& Hildebrandt basal salt medium ${ }^{1,2}$ & $1 x$ & $1 \mathrm{x}$ & $1 x$ \\
\hline Schenk \& Hildebrandt vitamin mixture ${ }^{1,2}$ & $1 x$ & $1 x$ & $1 x$ \\
\hline Sucrose ${ }^{1}$ & $1 \%(w / v)$ & $1 \%(w / v)$ & $2 \%(w / v)$ \\
\hline BAP (6-Benzylaminopurine) ${ }^{3}$ & - & $1 \mathrm{mg} / \mathrm{l}$ & - \\
\hline IBA (Indole-3-butyric acid) ${ }^{3}$ & - & $0.1 \mathrm{mg} / \mathrm{l}$ & $1 \mathrm{mg} / \mathrm{l}$ \\
\hline NAA (1-Naphthaleneacetic acid) ${ }^{3}$ & - & - & $0.1 \mathrm{mg} / \mathrm{l}$ \\
\hline MES (2-ethanesulfonic acid) ${ }^{3}$ & $3 \mathrm{mM}, \mathrm{pH}=5.8$ & $3 \mathrm{mM}, \mathrm{pH}=5.8$ & $3 \mathrm{mM}, \mathrm{pH}=5.8$ \\
\hline Daishin agar ${ }^{1}$ & - & $0.8 \%(\mathrm{w} / \mathrm{v})$ & $0.8 \%(\mathrm{w} / \mathrm{v})$ \\
\hline
\end{tabular}

Supplemental Table 8. GenelDs for all $P$. andersonii genes used in this study. GenelDs refer to $P$. andersonii gene models, which can be searched for on www.parasponia.org.

\begin{tabular}{cl}
\hline Name & GenelD \\
\hline PanEIN2 & PanWU01x14_asm01_ann01_090380 \\
\hline PanHK2 & PanWU01x14_asm01_ann01_002510 \\
\hline PanHK3 & PanWU01x14_asm01_ann01_222240 \\
\hline PanHK4 & PanWU01x14_asm01_ann01_103390 \\
\hline PanNSP1 & PanWU01x14_asm01_ann01_334190 \\
\hline PanSCL26 & PanWU01x14_asm01_ann01_236490 \\
\hline PanSCL34 & PanWU01x14_asm01_ann01_107700 \\
\hline PanSCL16 & PanWU01x14_asm01_ann01_130270 \\
\hline PanSHR & PanWU01x14_asm01_ann01_108080 \\
\hline PanNSP2 & PanWU01x14_asm01_ann01_157260 \\
\hline PanSCL28 & PanWU01x14_asm01_ann01_101430 \\
\hline PanSCL18 & PanWU01x14_asm01_ann01_295370 \\
\hline PanSCL17 & PanWU01x14_asm01_ann01_245480 \\
\hline PanSCL27 & PanWU01x14_asm01_ann01_245490 \\
\hline PanRAM1 & PanWU01x14_asm01_ann01_146390 \\
\hline PanD27 & PanWU01x14_asm01_ann01_194930 \\
\hline PanD27L1 & PanWU01x14_asm01_ann01_057040 \\
\hline PanD27L2 & PanWU01x14_asm01_ann01_143920 \\
\hline PanMAX1 & PanWU01x14_asm01_ann01_182160 \\
\hline PanUNK2 & PanWU01x14_asm01_ann01_211960 \\
\hline PanAGT1 & PanWU01x14_asm01_ann01_070680 \\
\hline- & PanWU01x14_asm01_ann01_040540 \\
\hline & \\
\hline & \\
\hline & \\
\hline &
\end{tabular}



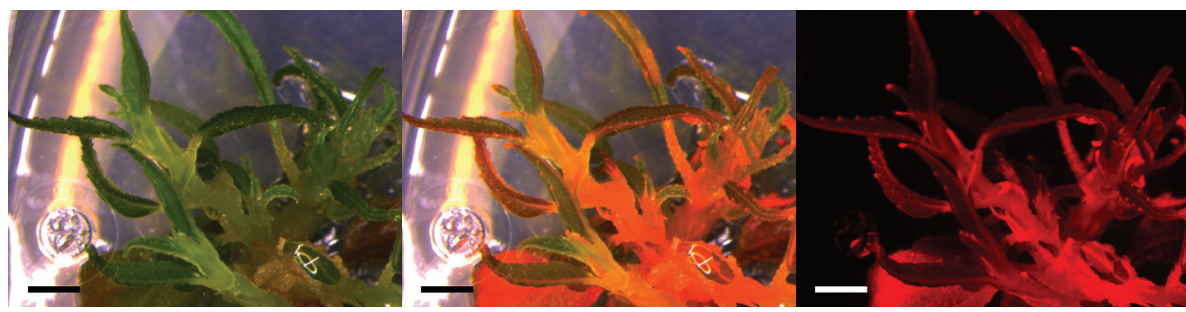

Supplemental Figure 1. P. andersonii transgenic shoots can be effectively propagated.

Transgenic $P$. andersonii shoots propagated in vitro. Images were taken six months after transformation. Scale bars are equal to $2.5 \mathrm{~mm}$. Shown from left to right are bright-field images, overlays of bright-field and DsRED fluorescence and DsRED fluorescence images.

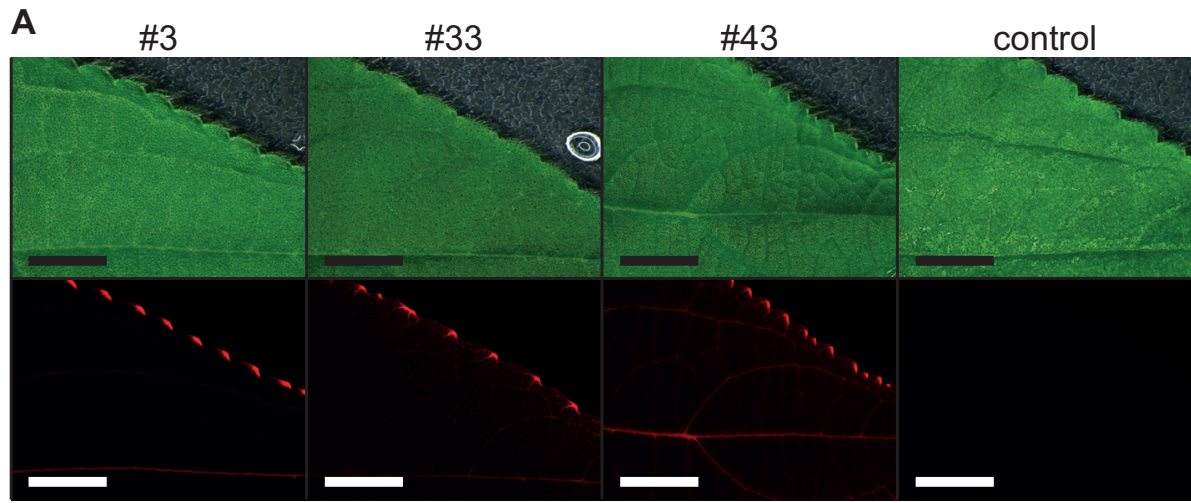

B

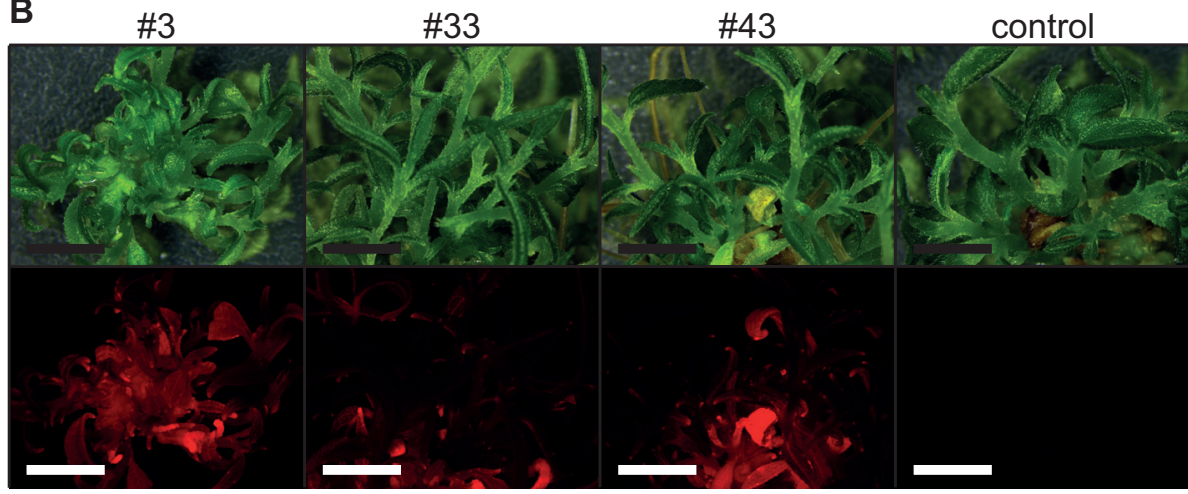

Supplemental Figure 2. Trans-genes remain stably integrated in the $P$. andersonii genome.

Bright field (top) and DsRED1 fluorescence (bottom) images of leaves harvested from mature trees grown under greenhouse conditions (A) or in vitro propagated material (B). Images are shown for transgenic lines 3, 33 and 43 and control plants not expressing the DsRED1 marker. Images were taken one year after transgenic lines were selected. Scale bars are equal to $5 \mathrm{~mm}$. 


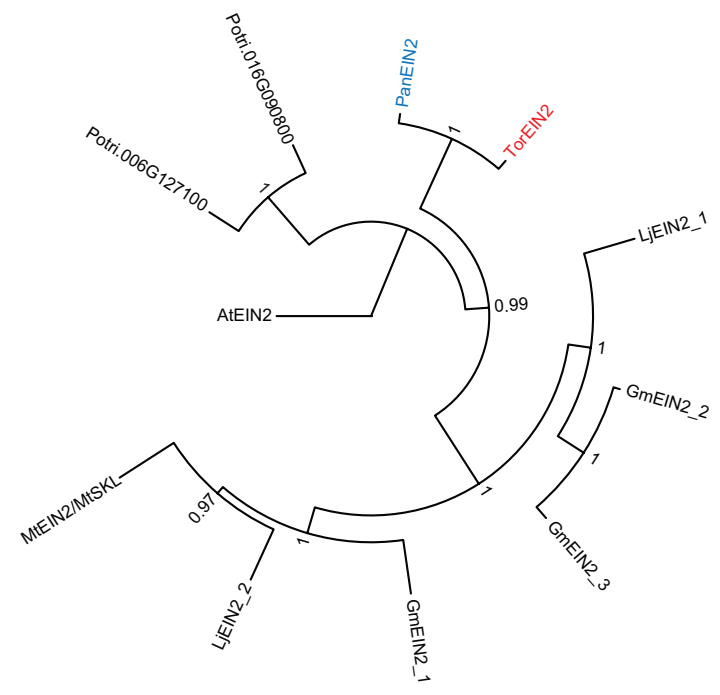

Supplemental Figure 3. Phylogeny of EIN2 proteins.

Phylogeny was reconstructed based on an alignment of EIN2 proteins from Arabidopsis thaliana (At), soybean (Glycine max, Gm), Lotus japonicus (Lj), Medicago truncatula (Mt), poplar (Populus trichocarpa, Potri), Parasponia andersonii (Pan) and Trema orientalis (Tor). Branch support is indicated by FastTree support values [78]. Terminals are labeled by their gene name or gene identifier. EIN2 proteins of $P$. andersonii and $T$. orientalis are highlighted in blue and red, respectively. Mid-point rooting was applied for better tree visualization. 


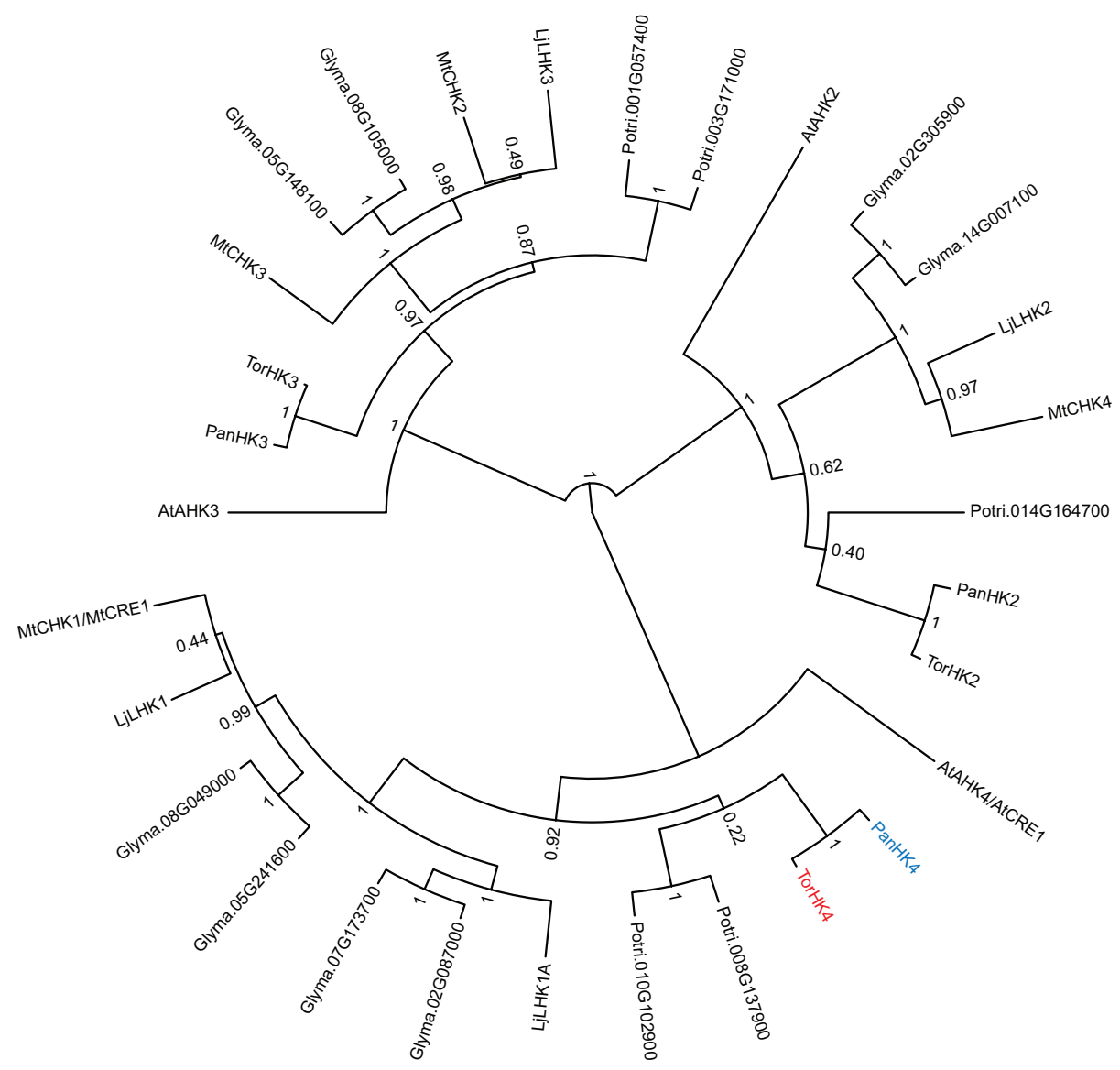

Supplemental Figure 4. Phylogeny of the histidine kinase cytokinin receptor family.

Phylogeny was reconstructed based on an alignment of cytokinin receptor proteins from Arabidopsis thaliana (At), soybean (Glycine max, Gm), Lotus japonicus (Lj), Medicago truncatula (Mt), poplar (Populus trichocarpa, Potri), Parasponia andersonii (Pan) and Trema orientalis (Tor). Branch support is indicated by FastTree support values [78]. Terminals are labeled by their gene name or gene identifier. The MtCRE1/LjLHK1 putative orthologues of $P$. andersonii and T. orientalis are highlighted in blue and red, respectively. Mid-point rooting was applied for better tree visualization. 


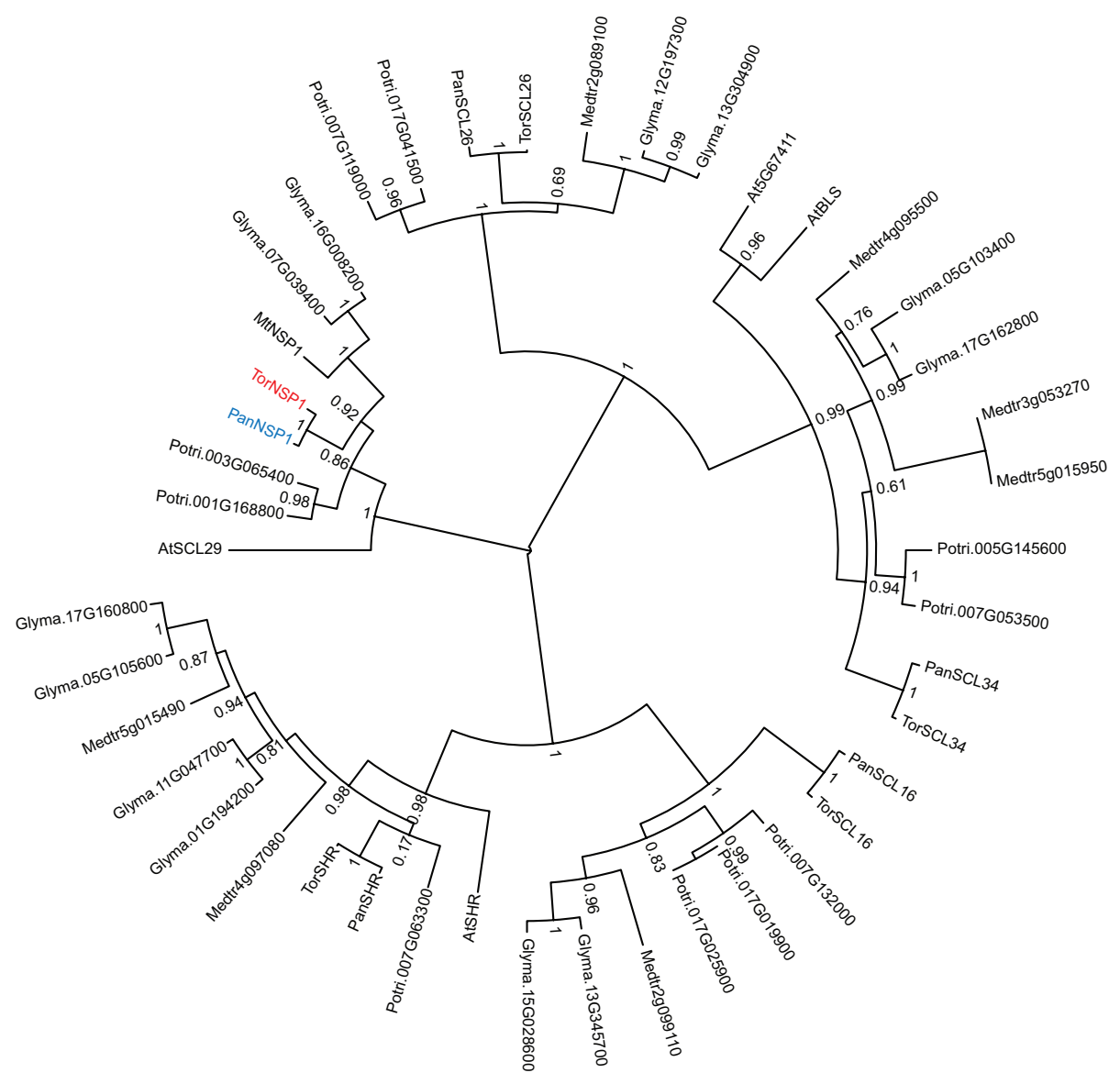

Supplemental Figure 5. Phylogeny of NSP1 and related GRAS proteins.

Phylogeny was reconstructed based on an alignment of GRAS proteins from Arabidopsis thaliana (At), soybean (Glycine max, Gm), Medicago truncatula (Mt), poplar (Populus trichocarpa, Potri), Parasponia andersonii (Pan) and Trema orientalis (Tor). Branch support is indicated by FastTree support values [78]. Terminals are labeled by their gene name or gene identifier. The MtNSP1 putative orthologues of $P$. andersonii and $T$. orientalis are highlighted in blue and red, respectively. Mid-point rooting was applied for better tree visualization. 


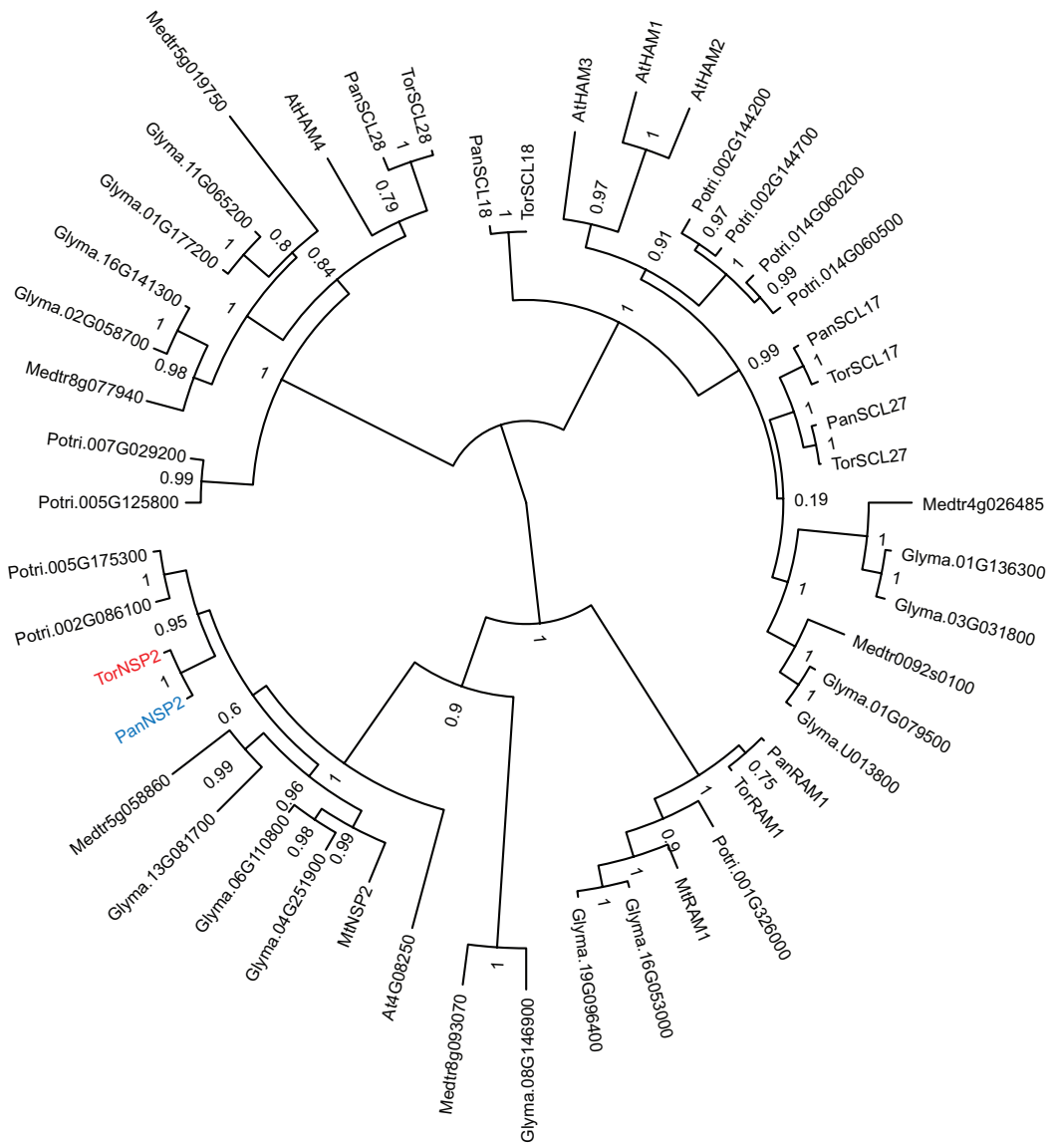

Supplemental Figure 6. Phylogeny of NSP2 and related GRAS proteins.

Phylogeny was reconstructed based on an alignment of GRAS proteins from Arabidopsis thaliana (At), soybean (Glycine max, Gm), Medicago truncatula (Mt), poplar (Populus trichocarpa, Potri), Parasponia andersonii (Pan) and Trema orientalis (Tor). Branch support is indicated by FastTree support values [78]. Terminals are labeled by their gene name or gene identifier. The MtNSP2 putative orthologues of $P$. andersonii and T. orientalis are highlighted in blue and red, respectively. Mid-point rooting was applied for better tree visualization. 
A

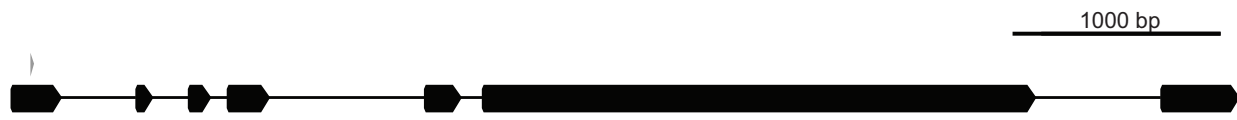

B

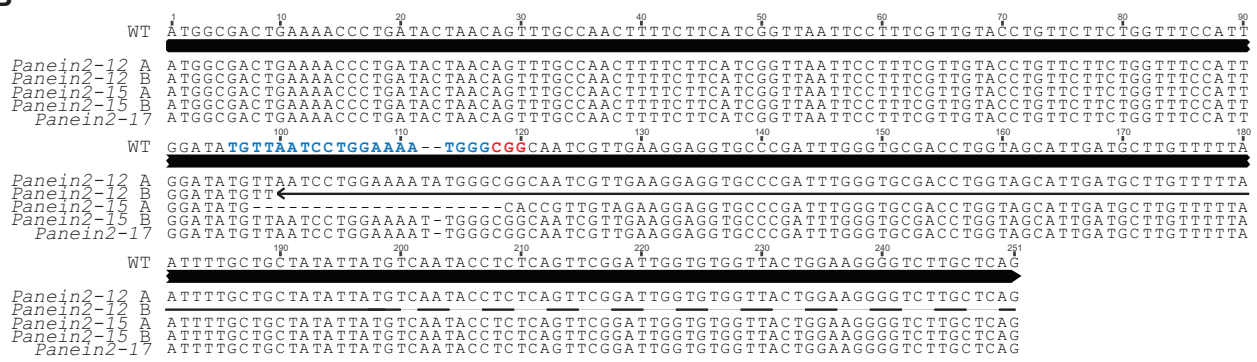

Supplemental Figures 7. CRISPR mutant alleles of Panein2 mutant lines.

(A) Schematic representation of PanEIN2 gene model. Indicated by a grey arrowhead is the location of the sgRNA target site.

(B) Sequence alignment of the first exon of PanEIN2 in wild type (WT) and Panein2 mutant lines. For biallelic mutant lines, both alleles are shown ( $A$ and $B$ alleles). Highlighted in blue and red are the sgRNA target site and PAM sequence, respectively. Arrow in sequence indicates a large (> $100 \mathrm{bp}$ ) insertion in Panein2-12 allele B.

A

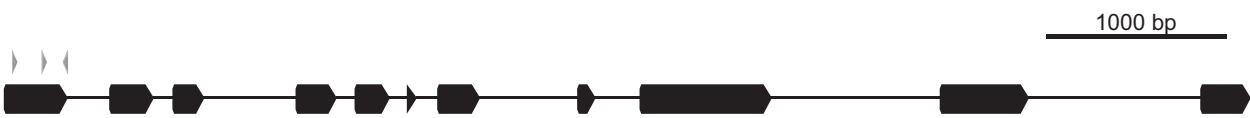

B

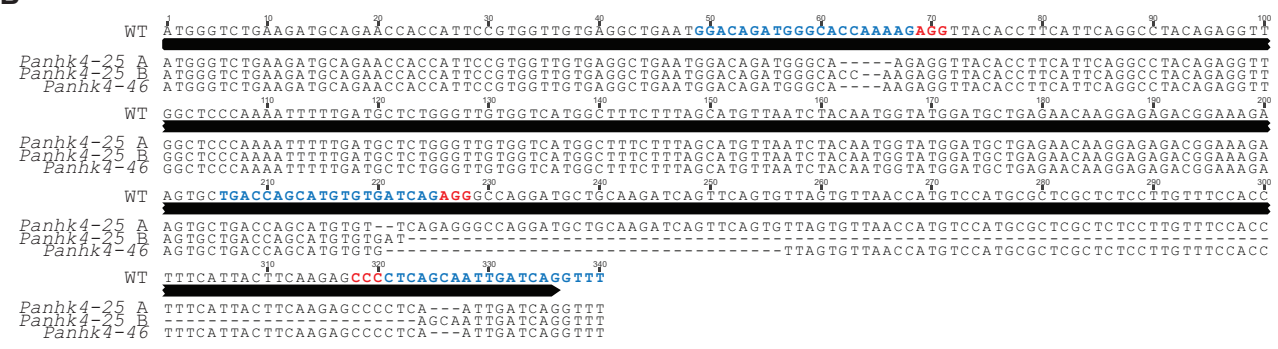

Supplemental Figures 8. CRISPR mutant alleles of Panhk4 mutant lines.

(A) Schematic representation of PanHK4 gene model. Indicated by grey arrowheads are the locations of three sgRNA target sites.

(B) Sequence alignment of the first exon of PanHK4 in wild type (WT) and Panhk4 mutant lines. For biallelic mutant lines, both alleles are shown ( $A$ and $B$ alleles). Highlighted in blue and red are the sgRNA target sites and PAM sequences, respectively. 
A

B

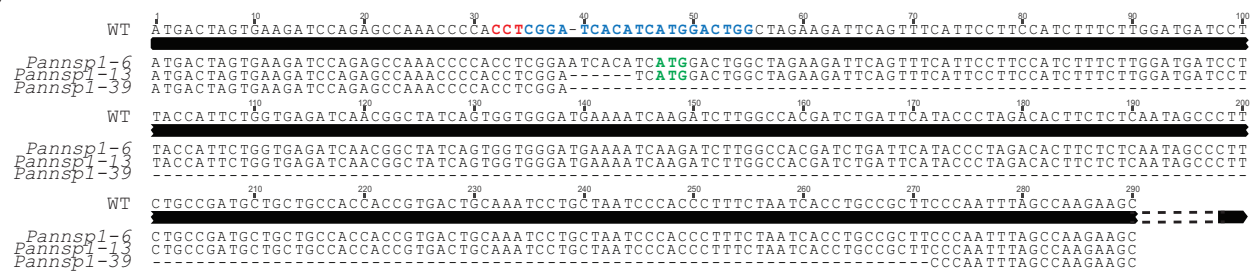

Supplemental Figures 9. CRISPR mutant alleles of Pannsp1 mutant lines.

(A) Schematic representation of PanNSP1 gene model. Indicated by a grey arrowhead is the locations of the sgRNA target site.

(B) Sequence alignment of the first part of PanNSP1 in wild type (WT) and Pannsp1 mutant lines. Highlighted in blue and red are the sgRNA target site and PAM sequence, respectively. Highlighted in green are in-frame ATGs present in Pannsp1-6 and Pannsp1-13.

A

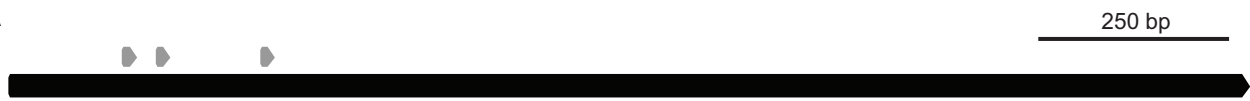

B

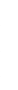
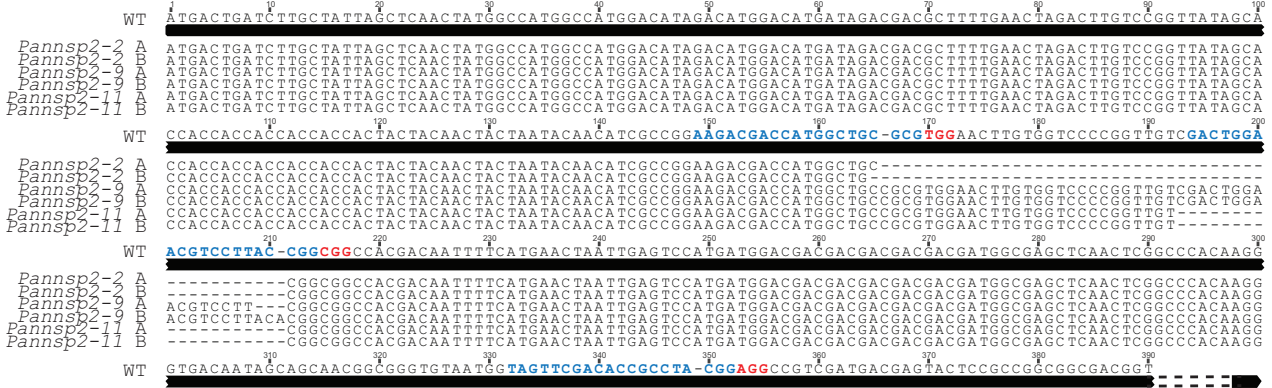

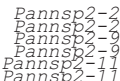
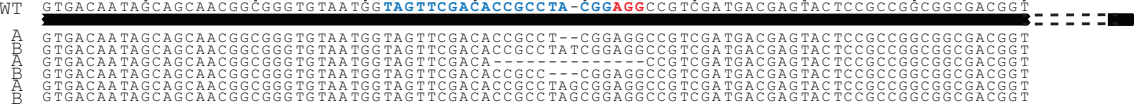

C

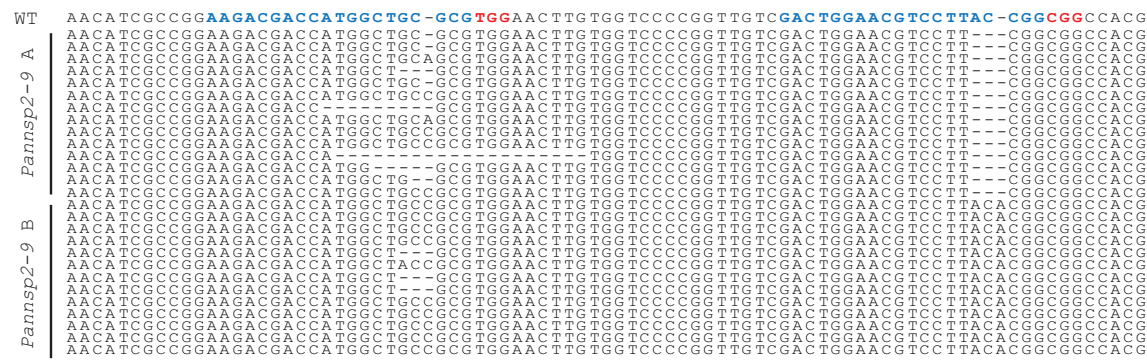




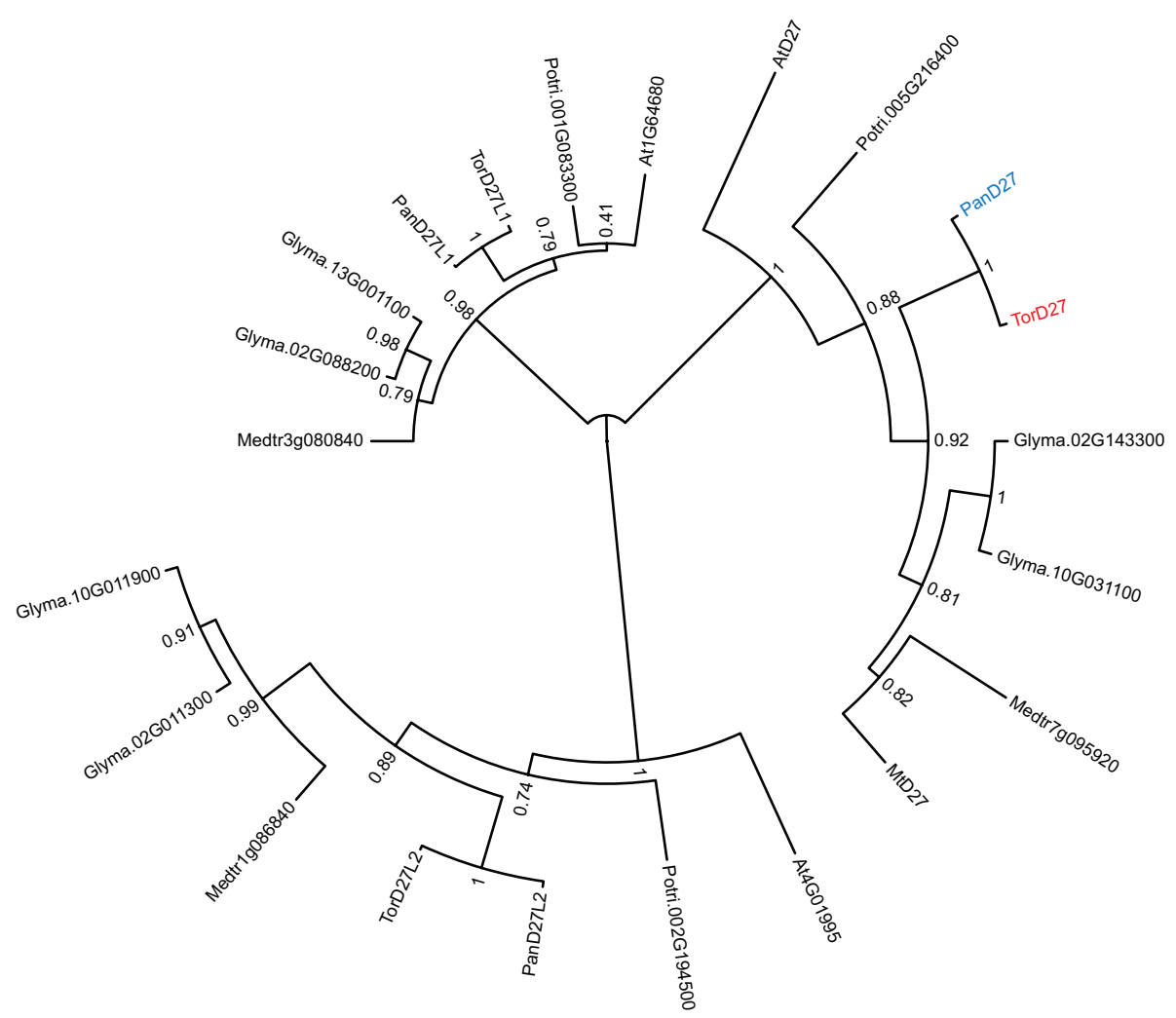

Supplemental Figures 11. Phylogeny of D27 and D27-like proteins.

Phylogeny was reconstructed based on an alignment of D27 and D27-like proteins from Arabidopsis thaliana (At), soybean (Glycine max, Gm), Medicago truncatula (Mt), poplar (Populus trichocarpa, Potri), Parasponia andersonii (Pan) and Trema orientalis (Tor). Branch support is indicated by FastTree support values [78]. Terminals are labeled by their gene name or gene identifier. The $P$. andersonii and T. orientalis putative orthologues of MtD27 are highlighted in blue and red, respectively. Mid-point rooting was applied for better tree visualization.

\section{Supplemental Figures 10. CRISPR mutant alleles of Pannsp2 mutant lines.}

(A) Schematic representation of PanNSP2 gene model. Indicated by grey arrowheads are the locations of three sgRNA target sites.

(B) Sequence alignment of the first part of the PanNSP2 gene in wild type (WT) and Pannsp2 mutant lines. For bi-allelic mutant lines, both alleles are shown (A and B alleles). Highlighted in blue and red are the sgRNA target sites and PAM sequences, respectively.

(C) Sequence alignment of part of the PanNSP2 gene in wild type (WT) and Pannsp2-9. The region depicted corresponds to the first two sgRNA target sites. Sequences from multiple individual Pannsp2-9 shoots are shown, to indicate that this line is chimeric for the first sgRNA target site. Highlighted in blue and red are the sgRNA target sites and PAM sequences, respectively. 


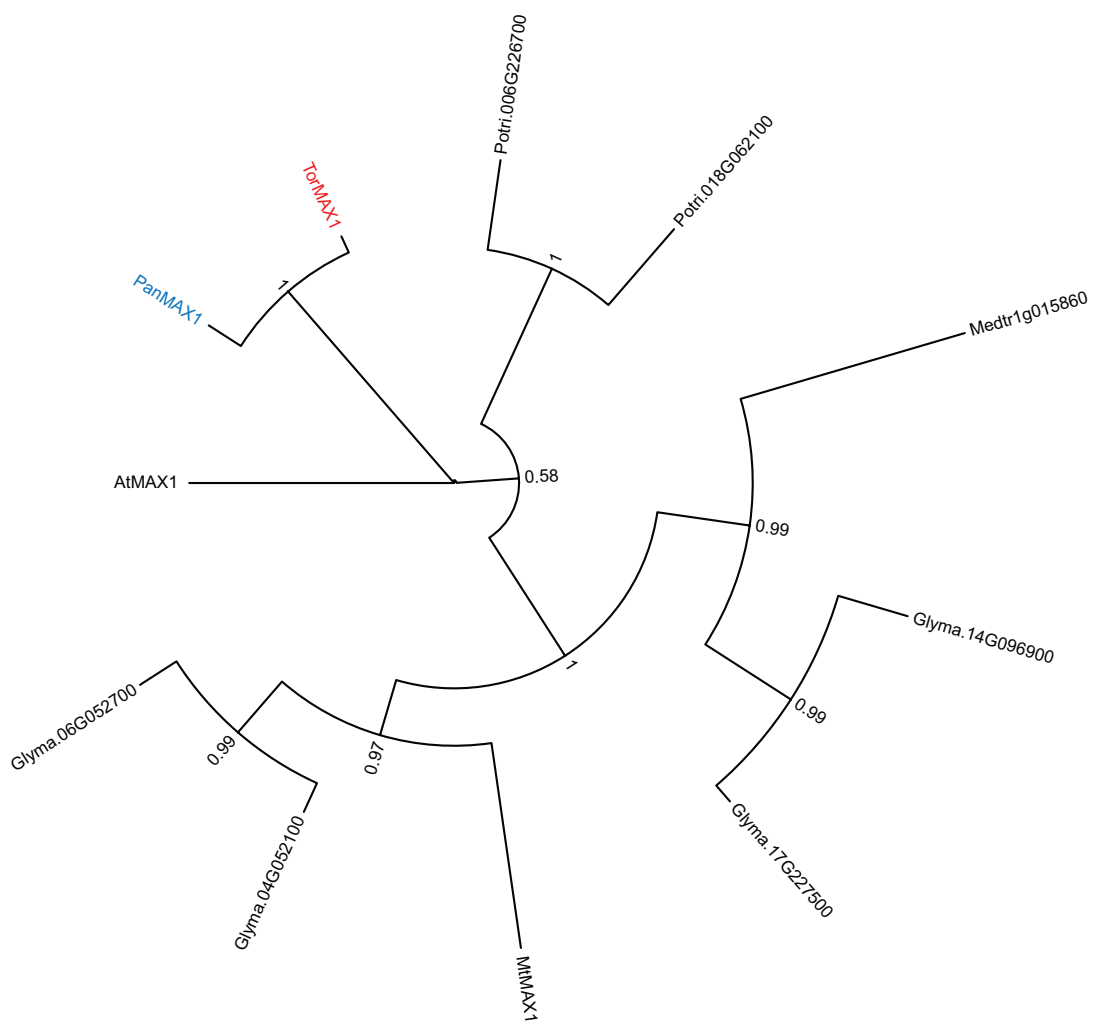

\section{Supplemental Figures 12. Phylogeny of MAX1 proteins.}

Phylogeny was reconstructed based on an alignment of MAX1 proteins from Arabidopsis thaliana (At), soybean (Glycine max, Gm), Medicago truncatula (Mt), poplar (Populus trichocarpa, Potri), Parasponia andersonii (Pan) and Trema orientalis (Tor). Branch support is indicated by FastTree support values [78]. Terminals are labeled by their gene name or gene identifier. The $P$. andersonii and T. orientalis putative orthologues of MtMAX1 are highlighted in blue and red, respectively. Mid-point rooting was applied for better tree visualization. 

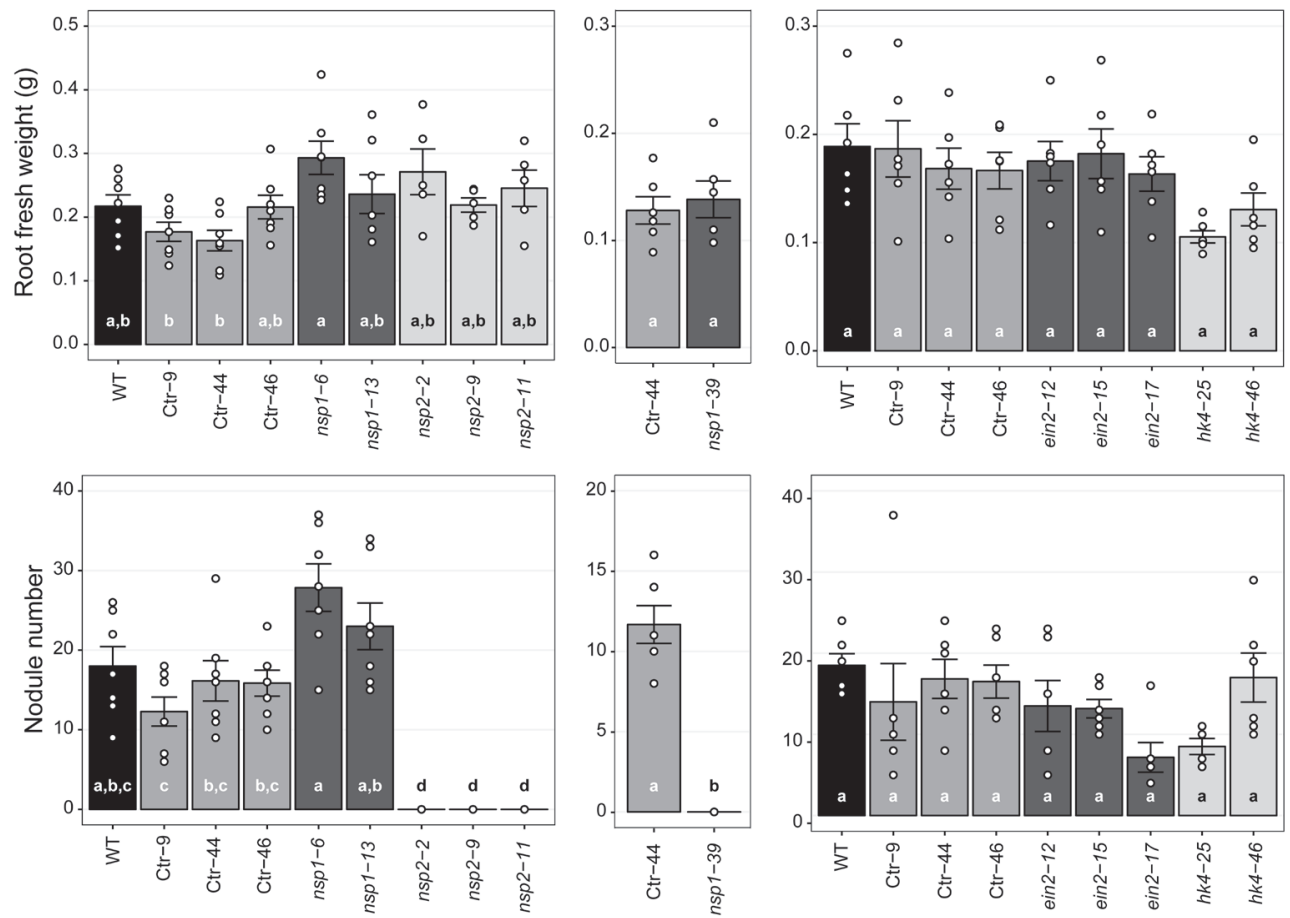

Supplemental Figure 13. Nodule formation on P. andersonii CRISPR/Cas9 mutant lines.

Nodule number and fresh root weight data belonging to Figure 7. Nodule number and root fresh weight was determined at one month post inoculation with Mesorhizobium plurifarium BOR2. Data represent means of 5-7 biological replicates \pm SEM. Dots represent measurement values of biological repeats. Different letters indicate statistical significance $(p<0.05)$ as determined by ANOVA in combination with Tukey post-hoc test. WT, wild type. 



\section{Chapter 6}

\section{Nodulating Parasponia and non-nodulating}

\section{Trema species harbor distinct root}

\section{transcriptomes}

Arjan van Zeijl, Robin van Velzen, Rens Holmer, Kerstin Guhl, Sidney Linders, Marijke Hartog, Jan Verver, Wouter Kohlen, Ton Bisseling and Rene Geurts 


\section{Abstract}

Parasponia represents the only non-legume lineage able to establish a nitrogen-fixing nodule symbiosis with rhizobium. Phylogenetic reconstruction showed that the Parasponia lineage is embedded in the non-nodulating genus Trema. This close genetic relationship suggests that both groups of species only recently diverged in nodulation ability. Here, we exploited this close phylogenetic relationship to determine to what extent differentiation in gene expression is correlated with the nodulation trait. By RNA-seq, we show that Parasponia and Trema harbor distinct root transcriptomes. This demonstrates that gene expression differentiation occurred between Parasponia and two Trema lineages. Among genes higher expressed in roots of Parasponia are several involved in mycorrhizal symbiosis as well as jasmonic acid biosynthesis. Measurements of hormone concentrations showed that Parasponia and Trema roots display a different jasmonic acid/salicylic acid balance. It is now a challenge to determine which of these differences are relevant in a symbiotic context, as mutants in jasmonic acid biosynthesis are unaffected in nodule development. 


\section{INTRODUCTION}

Plants require large amounts of mineral nitrogen for growth and development. However, in soil the amount of mineral nitrogen is often limiting. To cope with nitrogen limitation, some plants evolved an endosymbiotic relationship with nitrogen-fixing actinomycetes of the genus Frankia or with rhizobium bacteria [1, 2]. To allow this symbiosis to occur, these plants develop specialized organs on their roots, known as nodules. Nodules provide optimal physiological conditions for the fixation of atmospheric nitrogen by the microbial symbiont. Nitrogen-fixing endosymbioses are found among four taxonomic orders of flowering plants that together form the nitrogen-fixation clade [3-5]. Within this clade, nodulation is restricted to ten separate lineages that are scattered among many non-nodulating plant lineages [6]. The fact that nodulating lineages are phylogenetically related suggests that genetic adaptations that allowed these nitrogen-fixing symbioses have occurred in a common ancestor.

A major aim in symbiosis research is to understand the evolutionary trajectory towards such endosymbiosis. Nitrogen-fixing root nodules are best studied in legumes; especially in Lotus japonicus and Medicago truncatula, two species that serve as model. Legumes together with Parasponia (Cannabaceae) represent the only two lineages that can form nodules with rhizobium bacteria [3, 7-9]. Studies on $M$. truncatula and $L$. japonicus uncovered that the genetic network used to establish symbiosis with rhizobium was partly co-opted from that involved in the arbuscular mycorrhizal (AM) symbiosis [10-17]. To initiate symbiosis, both rhizobia and AM fungi excrete lipo-chitooligosaccharide (LCO) signals [18, 19]. These are perceived by LysM-type receptors at the plant root epidermis, which activates a signaling network largely shared between both symbioses [20-24]. Perception of rhizobium LCOs mitotically activates root cortical cells leading to nodule organogenesis $[25,26]$. Rhizobia intracellularly colonize nodule cells, but remain separated from the host cell cytoplasm by a host-derived membrane. Formation of this host-microbe interface membrane involves an exocytotic pathway that has also been co-opted from AM symbiosis [27-29].

To establish symbiosis, rhizobia and mycorrhizal fungi need to suppress plant immunity [30, 31]. In legumes, inoculation with incompatible rhizobium bacteria elevates salicylic acid (SA) levels [32,33]. SA is an important regulator of basal immunity against biotrophic microbes and acts antagonistically to jasmonic acid (JA)-induced signaling [34-36]. JA is mainly involved in defense against necrotrophs and is produced from $\alpha$-linolenic acid, which is released from galactolipids in the chloroplast membrane [35, 37]. Consecutive activity of 13S-lipoxynases (LOX), allene oxide synthases (AOS) and allene oxide cyclases (AOC) converts $\alpha$-linolenic 
acid into the JA precursor cis-(+)-12-oxophytodienoic acid (OPDA). OPDA is subsequently converted into JA and its bioactive conjugate jasmonoyl-L-isoleucine (JA-Ile) [37, 38]. In alfalfa (Medicago sativa), an increase in LOX activity is correlated with successful infection by rhizobium, suggesting a role for JA in the nodulation process [39]. Similarly, application of $0.1 \mu \mathrm{M}$ JA to roots of $L$. japonicus promotes rhizobial infection and root nodule formation, whereas higher concentrations generally inhibit nodule organogenesis $[40,41]$.

Generally, traits evolve due to a combination of gene duplication and subsequent neofunctionalization, gene loss as well as regulatory evolution. To unravel the evolutionary trajectory of a nitrogen-fixing endosymbiosis, we recently adopted Parasponia as a research model [42]. The Parasponia lineage is embedded in the non-nodulating Trema genus [42, 43]. This close phylogenetic relationship between species that differ in their ability to nodulate provides an excellent model to get insight is the genetic changes that are associated with root nodule formation. Analysis of sequenced genomes for three Parasponia and three Trema species revealed $>25,000$ orthologous gene models and only limited number of copy number variants between Parasponia and Trema [42]. This analysis also showed that Trema spp. independently lost several putative symbiosis genes that are considered to be essential for root nodule formation in Parasponia [42]. This suggests that Trema spp. no longer possess the potential to nodulate, or even have lost this trait in recent history. Irrespective of the evolutionary trajectory, the close relationship of Parasponia and Trema, which only recently diverged in nodulation behavior, provides an excellent model to determine to what extend gene expression differentiation is associated with the nodulation trait.

Here, we show that Parasponia and Trema harbor distinct root transcriptomes. This shows that gene expression differentiation occurred between both groups of species. Among the differentially expressed genes are several involved in mycorrhizal symbiosis and JA homeostasis. Measurements of root hormone content revealed that Parasponia roots contain higher OPDA and JA, but lower SA concentrations compared to Trema, indicating a difference in JA/SA balance. The biological significance of this remains currently unknown, as mutants in JA biosynthesis are unaffected in nodule development.

\section{Results}

\section{Parasponia and Trema Root Transcriptomes Are Distinct}

Nodulating Parasponia recently diverged from closely related non-nodulating Trema species. We questioned whether regulatory evolution occurred after the Parasponia-Trema 


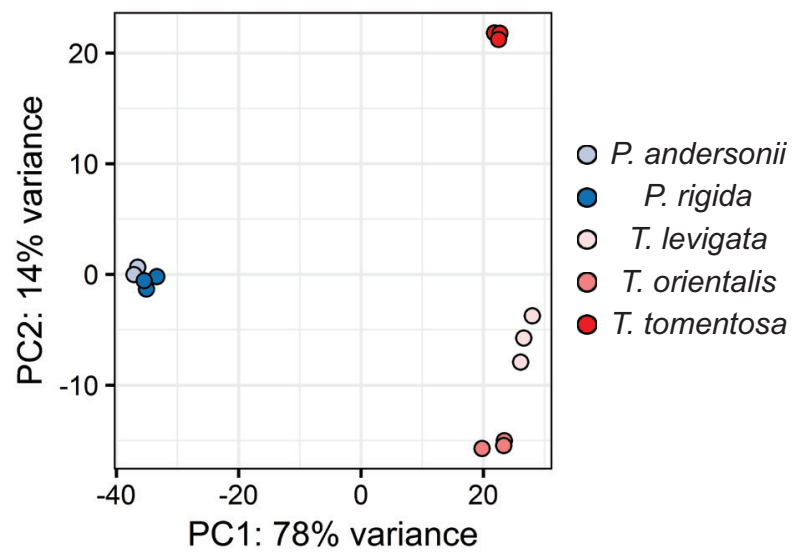

Figure 1. Parasponia and Trema harbor distinct root transcriptomes.

Principal component analysis (PCA) on gene expression values in roots of two Parasponia and three Trema species. PCA was performed on DESeq2 'regularized log' transformed read counts obtained after mapping of $P$. andersonii and $P$. rigida reads to the $P$. andersonii genome and $T$. levigata, $T$. orientalis and $T$. tomentosa reads against the $T$. orientalis genome. Each dot represents a biological replicate. Plotted are first two principal components (PC1 and PC2) and indicated is the percentage of explained variance by each principal component.

split. Therefore, we determined root transcriptomes of two Parasponia species as well as the closely-related sister species Trema levigata and the outgroup species Trema orientalis and Trema tomentosa [42] by RNA sequencing (RNA-seq). To this end, non-inoculated seedlings of all five species were grown in perlite watered with EKM medium ( $5 \mathrm{mM} \mathrm{KNO}_{3}$ ) [44]. When inoculated, this condition is conducive for Parasponia root nodule formation (Supplemental Figure 1). After four weeks of growth, RNA was isolated from a $\sim 5-10 \mathrm{~mm}$ region just above the root tip of young freshly growing roots. For analysis of RNA-seq data, we focused on 24,246 high-confidence orthologous gene pairs identified between the genomes of Parasponia andersonii and T. orientalis [42]. This allows direct comparison of gene expression values between Parasponia and Trema species. Principal component analysis (PCA) on DESeq2 'regularized log' transformed read counts separates Parasponia from Trema samples along the first principal component (PC1; Figure 1). This separation is not affected by cross-mapping of reads to either the $P$. andersonii or $T$. orientalis genome (Supplemental Figure 2). The separation along PC1 explains $\geq 75 \%$ of the observed variance, indicating substantial variation between Parasponia and Trema root transcriptomes (Figure 1 and Supplemental Figure 2). Including samples from $P$. andersonii and T. tomentosa plants obtained through in vitro propagation and grown under a different nitrogen regime $(0.375$ $\mathrm{mM} \mathrm{NH} \mathrm{NO}_{3}$ ) does not affect this separation, indicating robust differences (Supplemental 
Table 1. Classification of differentially expressed (fold change $>2, p<0.05$ ) genes in functional classes.

\begin{tabular}{ccc}
\hline Functional class & $\begin{array}{c}\text { Higher expressed in } \\
\text { Parasponia }\end{array}$ & $\begin{array}{c}\text { Lower expressed in } \\
\text { Parasponia }\end{array}$ \\
\hline Transcription factors & 9 & 2 \\
Symbiosis & 3 & 0 \\
Hormone homeostasis & 8 & 5 \\
Immunity & 14 & 7 \\
Enzymes & 39 & 65 \\
Transporters & 8 & 21 \\
Signaling & 12 & 17 \\
Cell wall & 3 & 3 \\
No annotation & 33 & 23 \\
Other & 50 & 68 \\
\hline Total & 179 & 211 \\
\hline
\end{tabular}

Figure 3). Therefore, we conclude that the root transcriptomes of two Parasponia species are distinct from that of the Trema sister species T. levigata, as well as the outgroup species T. orientalis and T. tomentosa.

Genes Lower Expressed in Parasponia Roots Are Enriched for Genes with a NoduleEnhanced Expression Profile

We questioned whether regulatory evolution between Parasponia and Trema is relevant in a symbiotic context. To this end, we first identified genes that show consistent differential expression between both groups of species. For this, we made use of both RNA-seq datasets described above. Based on a minimal fold change larger than two and an FDR-corrected $p$-value smaller than 0.05 , we identified 179 and 211 genes that are consistently higher or lower expressed in roots of Parasponia spp., respectively (Supplemental Tables 1-2). These differentially expressed genes were manually divided into nine functional classes, including symbiosis, transcription factors and hormone homeostasis (Table 1). This showed that the majority of differentially expressed genes encode enzymes involved in primary and secondary metabolism (Table 1).

The differentially expressed genes theoretically represent three, partly overlapping classes: I. genes that perform a symbiotic function, II. genes that exclusively perform non-symbiotic functions and III. genes that commit pleiotropic functions. To get first insight in which genes 

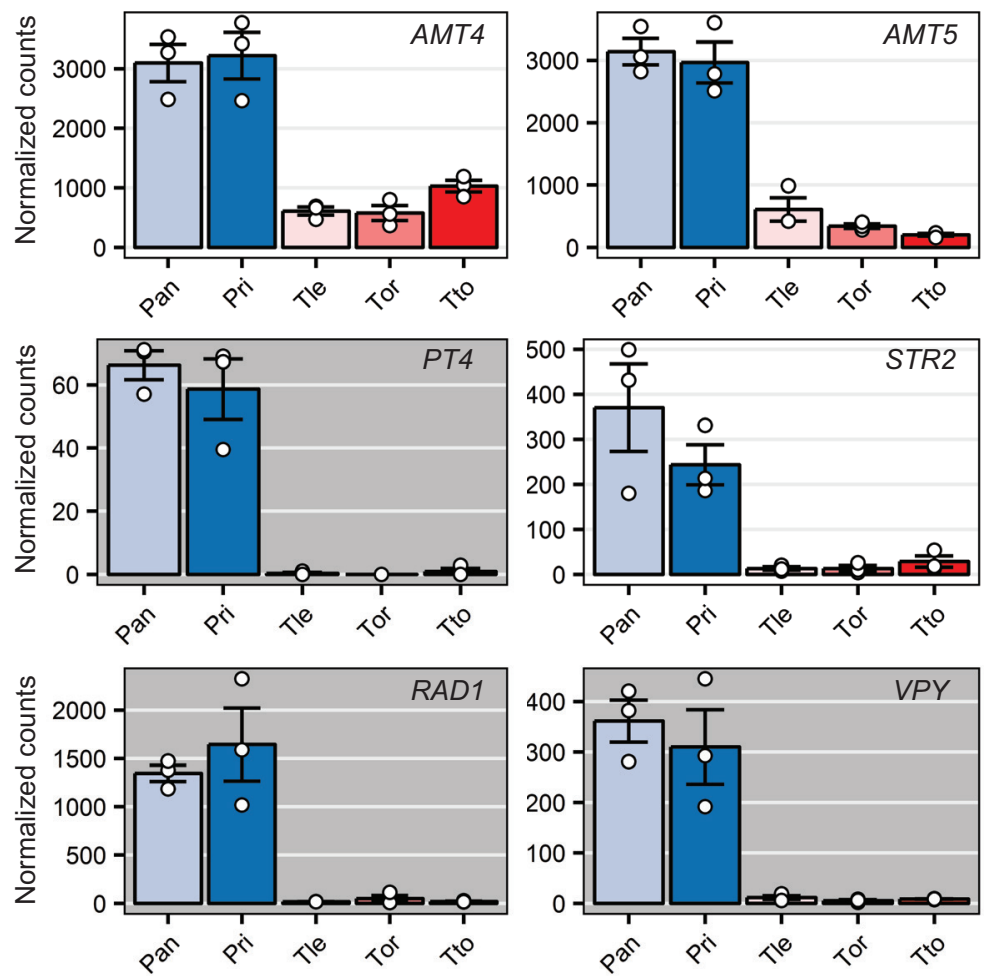

Figure 2. Expression of mycorrhizal marker genes is higher in roots of Parasponia.

Expression of the mycorrhizal marker genes AMT4, AMT5, PT4, STR2, RAD1 and VPY in roots of $P$. andersonii (Pan), P. rigida (Pri), T. levigata (Tle), T. orientalis (Tor) and T. tomentosa (Tto). Expression levels were calculated after mapping of $P$. andersonii and $P$. rigida reads to the $P$. andersonii genome and $T$. levigata, $T$. orientalis and $T$. tomentosa reads against the $T$. orientalis genome. Expression levels are presented as DESeq2 normalized read counts. Data represent means of 3 biological replicates \pm SEM. Dots represent expression values in each of the biological replicates. Grey panel highlighting of expression plots indicates differential expression (fold change $>2, p<0.05$ ), consistent across all comparisons.

may commit a symbiotic function (class I and III), we compared the list of differentially expressed genes to a published set of genes that show nodule-enhanced expression in $P$. andersonii [42]. This revealed that 18 of the genes that are higher expressed in Parasponia roots display a nodule-enhanced expression pattern (see Supplemental Table 1). This includes Vapyrin (VPY), which encodes a putative membrane trafficking protein required for AM fungal and rhizobium symbiosis in $M$. truncatula $[45,46]$. Among genes that are lower expressed in roots of Parasponia are 35 genes that are nodule-enhanced (see Supplemental Table 2). This represents significant enrichment ( $p<0.001$, hypergeometric test), suggesting that reduced expression in the root might potentiate genes to function in symbiosis. 
a-linolenic acid

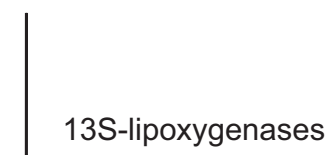

13-HPOT

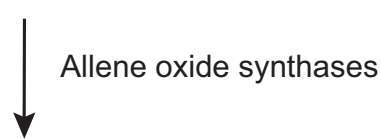

12,13-EOT

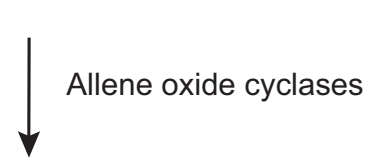

cis-(+)-12-OPDA

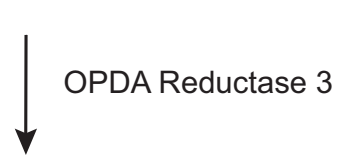

OPC-8:0
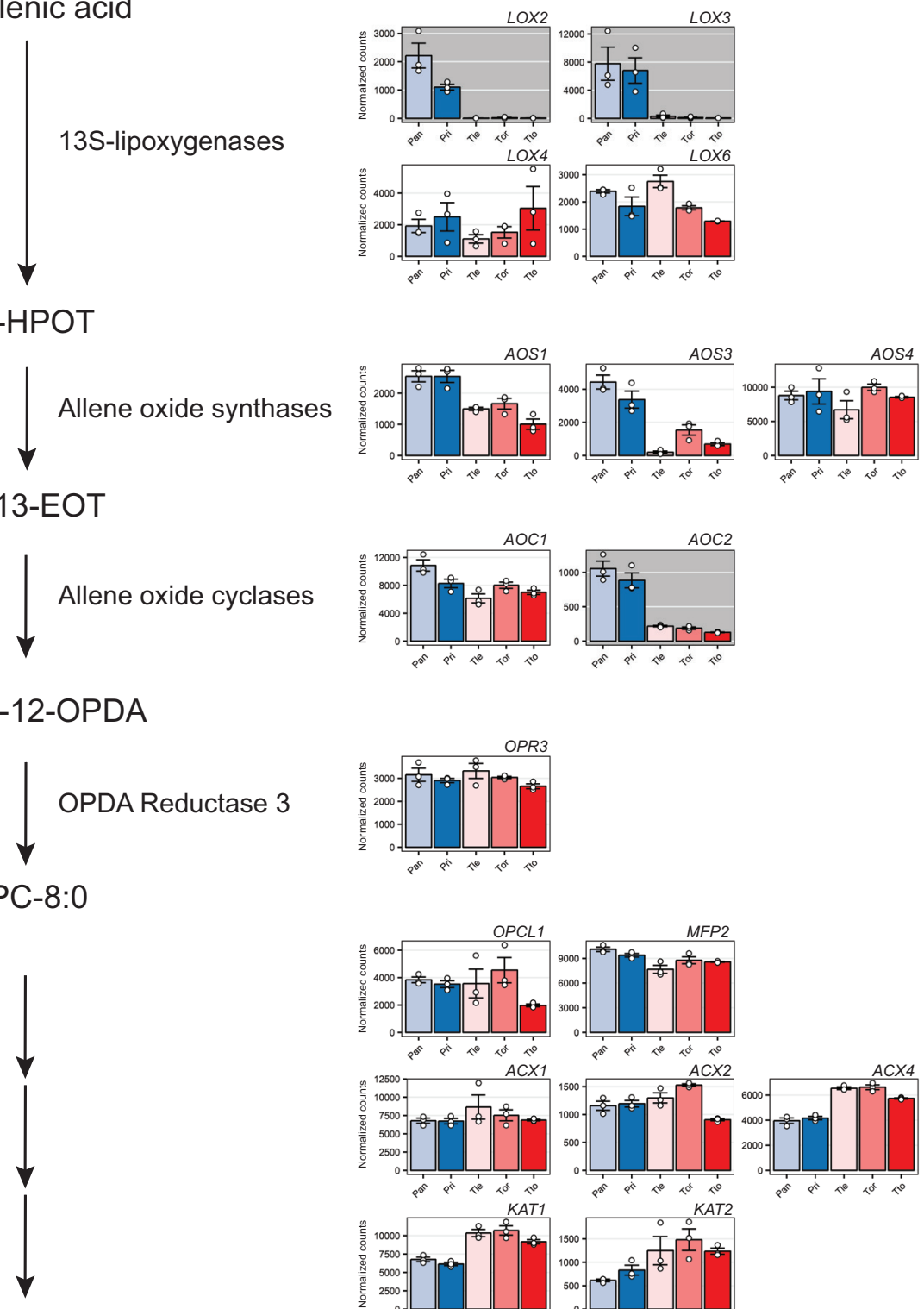


\section{AM Marker Genes Are Higher Expressed in Roots of Parasponia}

The genetic networks used by legumes and Parasponia to establish symbiosis with rhizobium have been partly co-opted from that involved in AM symbiosis [2, 24]. Interestingly, among the genes differentially expressed between Parasponia and Trema roots are three genes of which orthologs are shown to be required for AM symbiosis in other species (Table 1). These genes, VPY, the GRAS-type transcriptional regulator RAD1 and the phosphate transporter PT4 [47, 48], are all higher expressed in roots of Parasponia (Figure 2 and Supplemental Figure 4). As mentioned above, VPY also functions during legume-rhizobium symbiosis and displays a nodule-enhanced expression pattern in $P$. andersonii (Supplemental Figure 5). Conversely, PanRAD1 and PanPT4 are not expressed in nodules (Supplemental Figure 5), in line with specific functions in mycorrhization. VPY and PT4 are often used as marker genes for AM symbiosis in legumes, together with the GRAS-type transcriptional regulator RAM1, the glycerol-3-phosphate acyl transferase RAM2, the subtilase SbtM1, BCP1 encoding a blue copper-binding protein, the ammonium transporter LjAMT2;2 and the half-size ABC transporters STR and STR2 [49-55]. We questioned whether any of these additional AM marker genes are also higher expressed in Parasponia roots. The Parasponia-Trema orthologues of RAM1 and STR are not or very lowly expressed in non-inoculated roots, whereas a RAM2 orthologue shows equal expression between both species groups (data not shown). For SbtM1 and BCP1 no clear orthologues could be identified in ParasponiaTrema genomes. However, homologs of LjAMT2;2 (AMT4 and AMT5) and STR2 showed to be higher expressed in roots of Parasponia (Figure 2 and Supplemental Figure 4). Together, these data suggest that at least six AM marker genes, among which three transporters of phosphates or ammonium, are higher expressed in roots of Parasponia, when compared to Trema species.

\section{Parasponia and Trema Roots Contain a Different JA/SA Balance}

Changes to the hormone balance are known to cause pleiotropic effects and as such might alter symbiotic susceptibility as well as cause profound transcriptional changes $[40,56$, 57]. Among differentially expressed hormone-related genes, the 13S-lipoxygenase LOX2 is

Figure 3. JA biosynthesis genes $L O X 2, \angle O X 3$ and $A O C 2$ are higher expressed in roots of Parasponia. On the left, an overview of the JA biosynthesis pathway. On the right, expression of the corresponding JA biosynthesis genes in roots of $P$. andersonii (Pan), P. rigida (Pri), T. levigata (Tle), T. orientalis (Tor) and $T$. tomentosa (Tto). Expression levels were calculated after mapping of $P$. andersonii and $P$. rigida reads to the $P$. andersonii genome and $T$. levigata, $T$. orientalis and $T$. tomentosa reads against the $T$. orientalis genome. Expression levels are presented as DESeq2 normalized read counts. Data represent means of 3 biological replicates \pm SEM. Dots represent expression values in each of the biological replicates. Grey panel highlighting of expression plots indicates differential expression (fold change > $2, p<0.05)$, consistent across all comparisons. 

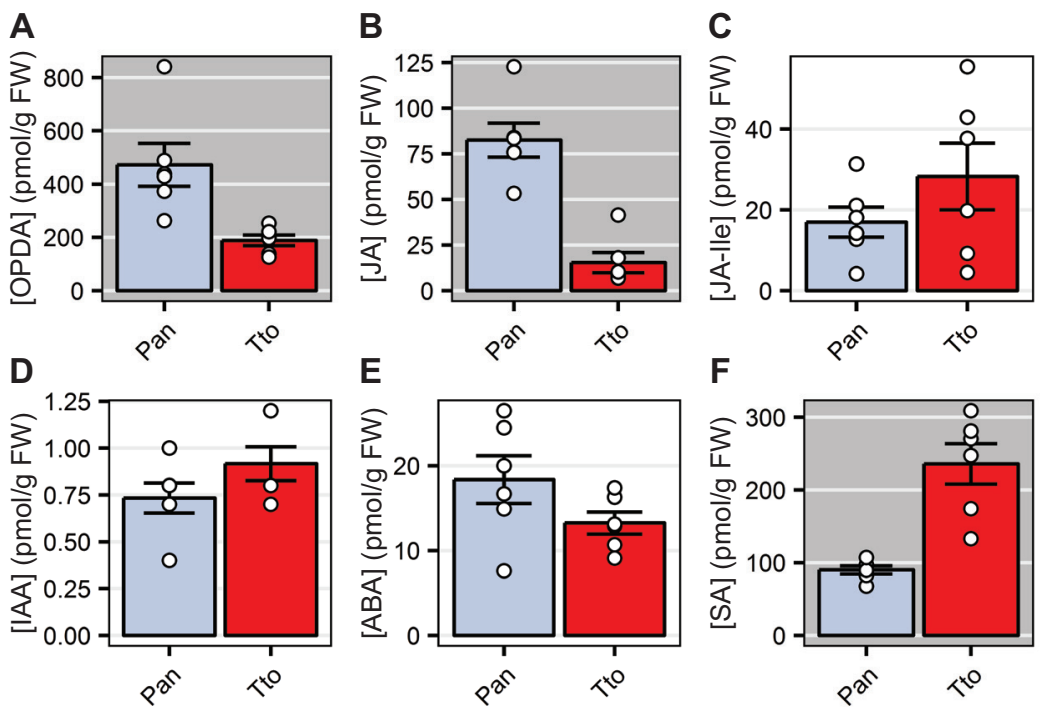

Figure 4. Parasponia and Trema roots contain different concentrations of OPDA, JA and SA

Concentrations of OPDA (A), JA (B), JA-Ile (C), IAA (D), ABA (E) and SA (F) in roots of $P$. andersonii (Pan) and T. tomentosa (Tto). Data represent means of 6 biological replicates \pm SEM. Dots represent expression values in each of the biological replicates. Grey panel highlighting indicates statistical significance ( $p<0.05$, Student's $t$-test). FW, fresh weight.

induced in nodules, suggesting a potential symbiotic function (Supplemental Figure 6). LOX2 is putatively orthologous to AtLOX2 from Arabidopsis, which is involved in JA biosynthesis [58]. Besides $L O X 2$, also its close paralogue, $L O X 3$, and the allene oxide cyclase $A O C 2$ are higher expressed in roots of Parasponia (Figure 3). Close inspection of the JA biosynthesis pathway, suggests that additional genes involved in production of the JA-precursor OPDA are also higher expressed in Parasponia roots, though not deemed significant across comparisons (Figure 3).

Higher expression of JA biosynthesis genes in roots of Parasponia could affect the root's hormone balance. Therefore, we measured concentrations of OPDA, JA, JA-Ile, salicylic acid (SA), abscisic acid (ABA) and indoleacetic acid (IAA) in roots of $P$. andersonii and $T$. tomentosa. Hormones were extracted from the same root zone as used for RNA-seq. In line with RNA-seq results, concentrations of OPDA and JA are 2.5- and 5-fold higher in roots of $P$. andersonii, respectively (Figure 4A-B). However, concentrations of the bio-active conjugate JA-Ile as well as ABA and IAA do not differ between both species (Figure 4C-E). Interestingly, SA concentrations are 2.5-fold lower in roots of $P$. andersonii (Figure 4F). This shows that young root segments of Parasponia and Trema harbor a distinct JA/SA balance. 
A
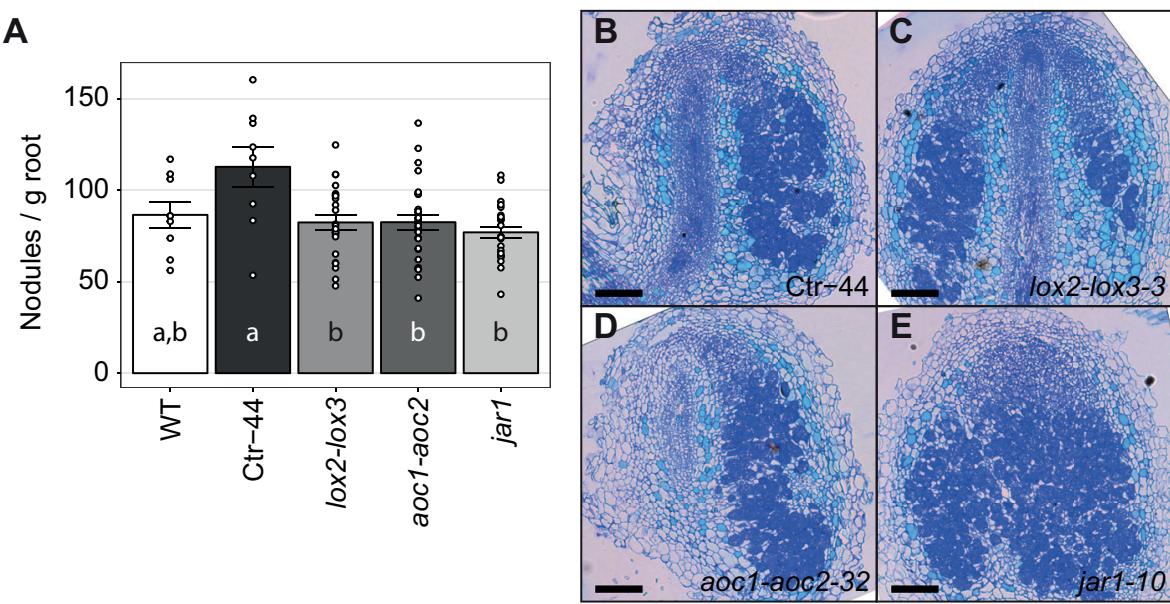

Figure 5. JA deficiency does not affect nodulation ability.

(A) Nodule formation on JA biosynthesis mutants at 6 weeks after inoculation with M. plurifarium BOR2. Data represent means of 9-29 biological replicates \pm SEM. Dots represent individual data points. For JA mutants, nodulation data was combined from 3-4 individual transgenic lines, each containing 6-8 biological replicates. Different letters on bars indicate statistical significance $(p<0.05$, ANOVA with Tukey post-hoc test). Data for each of the individual lines can be found in Supplemental Figure 10 , which also contains data on nodule number per plant and root fresh weight. This data was used to calculate the data presented here. WT, wild-type P. andersonii; Ctr-44, transgenic control line expressing the Cas9 gene only [Chapter 5 ].

(B-E) Longitudinal nodule sections through a transgenic control nodule (B), nodule formed on Panlox2-lox3 mutant line 3 (C), nodule formed on Panaoc1-aoc2 mutant line 32 (D) and nodule formed on Panjar1 mutant line 10 (E). Bars represent $150 \mu \mathrm{m}$.

\section{JA Deficiency Does Not Affect Rhizobium Symbiosis in Parasponia}

To determine the role of JA in Parasponia-rhizobium symbiosis, we generated PanLOX2PanLOX3 and PanAOC1-PanAOC2 double mutant lines and PanJAR1 single mutant lines, using CRISPR/Cas9 [Chapter 5]. Among these, Panaoc1-Panaoc2 double mutants are expected to be OPDA and JA deficient and Panjar1 mutants to be unable to convert JA into JA-Ile. An overview of mutant lines can be found in Supplemental Figure 7-9. To determine symbiotic mutant phenotypes, mutant lines were inoculated with Mesorhizobium plurifarium BOR2 [42]. After six weeks, nodule number was examined, which revealed no difference from wild type (Figure 5A and Supplemental Figure 10). To determine whether mutant nodules might be affected in intracellular colonization by rhizobium, we sectioned $>10$ nodules of Panlox2-Panlox3-3, Panaoc1-Panaoc2-32 and Panjar1-10. This showed that mutant nodules contain a wild-type cyto-architecture and are fully infected by rhizobium bacteria (Figure 5B-E). Therefore, mutant analysis indicates that JA deficiency does not affect Parasponiarhizobium symbiosis. 


\section{Discussion}

Parasponia and Trema represent closely related species with opposing symbiotic phenotypes. Genome comparisons revealed only limited number of copy number variants between both groups of species [42]. Here, we performed comparative transcriptome analysis between two Parasponia and three Trema species. This showed that Parasponia and Trema harbor distinct root transcriptomes, suggesting extensive regulatory evolution. Amongst others, this affected root hormone homeostasis. Expression analysis as well as hormone quantification showed that Parasponia and Trema roots harbor a different JA/SA balance. However, this difference does not appear to be essential for Parasponia root nodule formation, as JAdeficient mutants are unaffected in nodule development. Therefore, it is now a challenge to determine whether the transcriptional differences between Parasponia and Trema are relevant in a symbiotic context

PCA analysis on root expression values separated Parasponia from Trema samples, indicating that both groups of species harbor distinct root transcriptomes. The difference in transcriptomes is not correlated to phylogenetic distance, as T. levigata is more closely related to Parasponia than to T. orientalis or T. tomentosa [42]. This suggests that the 'core' Trema root transcriptome might better represent the ancestral state. Therefore, it is most parsimonious that regulatory evolution occurred in the most recent common ancestor of all Parasponia species, resulting in transcriptional divergence from Trema. However, Trema species independently lost genes that are putatively essential for rhizobium symbiosis in Parasponia, which has led to the hypothesis that Trema once possessed the ability to nodulate [42]. Therefore, it cannot be excluded that the transcriptional differences between Parasponia and Trema are due to parallel regulatory evolution in independent Trema lineages. However, this assumes that the transcriptional differences between both groups of species are directly or indirectly correlated to nodulation capacity. An overrepresentation of nodule-enhanced genes among differentially expressed genes between Parasponia and Trema roots, provides some support for this assumption.

Hormone measurements showed a higher concentration of OPDA and JA in roots of $P$. andersonii compared to T. tomentosa. In contrast, SA concentrations were higher in roots of T. tomentosa, indicating a shift in JA/SA balance. Higher expression of JA biosynthesis genes in roots of $P$. andersonii and $P$. rigida is consistent with higher OPDA and JA concentrations in $P$. andersonii roots, and suggests that the difference in OPDA and JA concentrations is consistent between Parasponia and Trema species. Whether the same applies to SA is difficult to predict. JA and SA function largely antagonistically and extensive cross-talk occurs 
between both hormone signaling pathways [34-36]. In Arabidopsis, basal SA concentrations are increased in the JA-deficient dde2 mutant, mutated in allene oxide synthase [59]. This implies that in wild-type Arabidopsis, JA signaling reduces SA concentrations. Therefore, it is possible that reduced basal SA levels in roots of Parasponia result from increased JA biosynthesis. Conversely, reduced SA concentrations could relief inhibition of JA signaling, promoting JA biosynthesis through positive feedback [60, 61].

A reduction in root SA concentrations could be relevant in a symbiotic context. SA functions in defense against biotrophic microbes [35] and therefore likely affects infection by rhizobia. In legumes, infection by incompatible rhizobium bacteria triggers an increase in SA concentration, whereas during a compatible interaction SA levels remain unaffected or are even slightly reduced [32, 33, 62]. We analyzed Parasponia mutants affected in JA biosynthesis and were unable to detect a nodulation phenotype. Whether in these mutants SA concentrations were increased to similar concentration as detected in $T$. tomentosa roots remains to be examined. Therefore, the ecological significance of a change in JA/SA balance between Parasponia and Trema roots remains unclear.

The absence of a nodulation phenotype for Parasponia JA mutants is consistent with studies in legumes. RNAi-mediated silencing of multiple LOX-encoding genes in soybean (Glycine max) or MtAOC1 in Medicago truncatula did not affect nodule development [63, 64]. Studies in L. japonicus, however, suggest a role for JA in controlling nodule number under shaded conditions. On wild-type $L$. japonicus grown under low red/far-red light conditions or in a phytochrome $B(p h y B$ ) mutant, nodule number is reduced [41]. Analysis of the phyB mutant showed that it contained reduced concentrations of JA-lle. Addition of low JA concentrations to the phy $B$ mutant or wild-type plants grown under low red/far-red rescued the nodulation phenotype [41]. This suggest that under specific environmental conditions, JA is involved in regulation of nodulation, though is not essential for nodule development.

In roots of Parasponia, we detected higher expression of several genes commonly used as markers for mycorrhizal symbiosis. This included RAD1, AMT4, AMT5, PT4, STR2 and VPY. RAD1 is closely related to RAM1 and both GRAS proteins form a protein complex together with DELLA proteins $[48,65,66]$. Over-expression of RAM1 or a stabilized version of $M$. truncatula DELLA2 (della2- $\triangle 18$ ) can induce expression of most, if not all, of the AM marker genes $[51,65,66]$. In non-inoculated Parasponia or Trema roots RAM1 is not expressed. However, it might be possible that RAD1, either alone or in combination with DELLAs, is responsible for the induction of AMT4, AMT5, PT4, STR2 and VPY expression in roots of Parasponia. Besides induced by mycorrhizal signaling, RAD1 expression is induced by 
phosphate starvation in L. japonicus root tips [48]. Phosphate starvation also stimulates accumulation of DELLA proteins in roots, through a reduction in gibberellic acid content [67]. Although highly speculative, it could be hypothesized that higher expression of AM marker genes in Parasponia roots, results from constitutive phosphate starvation signaling. In support of this hypothesis, phosphate starvation has been shown to increase JA levels in Arabidopsis roots and leaves [68]. Additionally, phosphate stress signaling has been shown to shift the JA/SA signaling balance in favor of JA response [69].

Trema are successional pioneering tree species that occur pantropically. They grow in areas where vegetation has been cleared due to natural or man-made disturbances, such as landslides, volcanic ash deposits and forest gaps [70-72]. Parasponia grows in similar habitats but its distribution is restricted to the Malay Archipelago and Papua New Guinea [73]. Parasponia seems to prefer nitrogen-poor eroded soils and is capable of forming dense stands on fresh deposits of volcanic ash $[72,73]$. Besides scarce in nitrogen, Parasponia habitats are often low in phosphorus as well as other essential nutrients [74, 75]. To support plant growth in nutrient-limited environments, plants interact with soil microbes, including mycorrhizal fungi and rhizobium bacteria. Phosphate limitation is an important regulator of AM symbiosis [76]. Therefore, constitutive phosphate starvation and/or higher expression of AM marker genes could promote mycorrhizal colonization of Parasponia roots. Experiments to compare mycorrhization efficiency between Parasponia and Trema species have failed to detect differences (Roswanjaya et al., personal communication). However, these experiments were done under relatively high inoculation density, which could mask mild differences in mycorrhization efficiency. In Arabidopsis, salicylic acid and phosphate stress signaling have been shown to function as important regulators of root microbiome composition $[69,77]$. Therefore, it is possible that root transcriptome differences between Parasponia and Trema are correlated to different microbiome dependencies. Additionally, in L. japonicus, nitrogenfixation has been shown to drastically affect root microbiome composition [78]. However, whether this directs evolution in pathways regulating microbiome assembly is unknown.

Taken together, we have shown that Parasponia and Trema roots harbor distinct transcriptomes and display a difference in root hormone balance. These differences could represent essential adaptations required for symbiosis establishment or indirectly result from differences in niche specialization. Therefore, unveiling the evolutionary drivers underlying the differences between Parasponia and Trema roots could provide insight in the ecological consequences of nitrogen-fixation. 


\section{Methods}

\section{Plant Materials and Growth Conditions}

For RNA-seq, seeds of Parasponia andersonii WU1, Parasponia rigida WU20, Trema levigata WU50, Trema orientalis RG33 and Trema tomentosa WU10 were germinated as previously described $[42,79]$. After approximately two weeks, seedlings were transferred to sterile 1 L crystal-clear polypropylene containers (OS140BOX, Duchefa Biochemie, The Netherlands) half-filled with agraperlite (Maasmond-Westland, The Netherlands) and watered with modified EKM medium ( $3 \mathrm{mM}$ MES $\left(\mathrm{C}_{6} \mathrm{H}_{13} \mathrm{NO}_{4}\right.$ ) pH 6.6, $2.08 \mathrm{mM} \mathrm{MgSO} 4^{\prime}, 0.88 \mathrm{mM} \mathrm{KH}_{2} \mathrm{PO}_{4^{\prime}}$ $2.07 \mathrm{mM} \mathrm{K}_{2} \mathrm{HPO}_{4}, 1.45 \mathrm{mM} \mathrm{CaCl}_{2}, 0.70 \mathrm{mM} \mathrm{Na}_{2} \mathrm{SO}_{4^{\prime}} 5 \mathrm{mM} \mathrm{KNO}_{3^{\prime}} 15 \mu \mathrm{M}$ Fe-citrate, $6.6 \mu \mathrm{M}$ $\mathrm{MnSO}_{4}, 1.5 \mu \mathrm{M} \mathrm{ZnSO}_{4}, 1.6 \mu \mathrm{M} \mathrm{CuSO}_{4}, 4 \mu \mathrm{M} \mathrm{H}_{3} \mathrm{BO}_{3}, 4.1 \mu \mathrm{M} \mathrm{Na}_{2} \mathrm{MoO}_{4}$ ) [44]. Plants were grown for 3-4 weeks in a climate room set at $28^{\circ} \mathrm{C}, 16 \mathrm{~h} / 8 \mathrm{~h}$ day/night, prior to tissue isolation.

For the second RNA-seq experiment, as well as hormone quantification and mutant analysis, in vitro propagated plantlets were used. In vitro propagation was performed as previously described [80]. Plants were grown under similar conditions as described above, with the modification that the nitrogen concentration in EKM medium was changed to $0.375 \mathrm{mM}$ $\mathrm{NH}_{4} \mathrm{NO}_{3}$. For nodulation assays, EKM was inoculated with Mesorhizobium plurifarium BOR2 $\left(\mathrm{OD}_{600}=0.025\right)[42]$. Nodulation was assessed six weeks post inoculation.

\section{RNA Sequencing}

For RNA isolation, tissue was harvested from a $\sim 5-10 \mathrm{~mm}$ region just above the root tip of young freshly growing roots and snap-frozen in liquid nitrogen. Material from $\sim 5$ plants was combined to form a single biological replicate. RNA was isolated as previously described [42]. Library preparation and RNA sequencing was conducted by B.G.I. (Shenzhen, China). Paired-end 100 bp reads were mapped against the $P$. andersonii WU1.14 and T. orientalis RG33.2 reference genomes [42] using HISAT2 (version 2.02) [81] using an index that includes exon and splice site information in the RNA-seq alignments. Mapped reads were assigned to transcripts with featureCounts (version 1.5.0) [82]. Principal component analysis, normalization and differential gene expression analysis were performed with DESeq2 (version 1.14.1) [83]. Analysis was based on 24,246 high-confidence 1-on-1 orthologue pairs identified between the genomes of $P$. andersonii and T. orientalis [42]. Differentially expressed genes were selected based on a fold-change $>2$ and an FDR-corrected $p$-value < 0.05 across pair-wise comparisons. Pair-wise comparisons were conducted between each Parasponia and Trema species (six comparisons in total) and between the $P$. andersonii and T. tomentosa RNA-seq samples obtained from in vitro propagated plantlets. To account for artefacts resulting from mapping to different genomes, reads of all species were mapped 
to both the $P$. andersonii and T. orientalis genomes. Differential expression was calculated after mapping of Parasponia reads to the P. andersonii genome and Trema reads to the $T$. orientalis genome, as well as after mapping of reads from both Parasponia and Trema to the $P$. andersonii genome or the $T$. orientalis genome. Genes were considered differentially expressed when they passed selection criteria after each of these three mapping strategies. For plotting of expression graphs, DESeq2 normalized count data obtained after mapping of Parasponia reads to the $P$. andersonii genome and Trema reads to the $T$. orientalis genome were used. Expression levels in $P$. andersonii nodules are obtained from van Velzen et al. [42]. Significant overrepresentation of nodule-enhanced genes among differentially expressed genes is calculated based on the hypergeometric probability.

\section{Hormone Extraction}

Hormone purification was performed using an adapted protocol based on Dobrev and Kaminek [84]. For extraction, $\sim 20 \mathrm{mg}$ of snap-frozen root material was used. Tissue was ground to a fine powder using 3-mm stainless steel beads at $50 \mathrm{~Hz}$ for $1 \mathrm{~min}$ in a Tissuelyser LT (Qiagen, Germantown, USA). Ground root samples were extracted with $1.5 \mathrm{ml}$ of $100 \%$ methanol containing $1 \mathrm{M}$ formic acid and $1 \mu \mathrm{M}$ of stable-isotope labelled internal standards (see Supplemental Table 3). Samples were vortexed and sonicated for $10 \mathrm{~min}$ in a Branson 3510 ultrasonic bath (Branson Ultrasonics, USA) and left rotating over night at $4^{\circ} \mathrm{C}$ for extraction. Samples were centrifuged for $10 \mathrm{~min}$ at $2500 \mathrm{~g}$ and the liquid phase was carefully transferred to a $4 \mathrm{ml}$ amber glass vial. Samples were re-extracted by rotating for $1 \mathrm{~h}$ at $4^{\circ} \mathrm{C}$ with $1.0 \mathrm{ml}$ of $80 \%$ methanol containing $1 \mathrm{M}$ formic acid. Both fractions were combined and dried in a speedvac concentrator (Savant SC210A) coupled tot a refrigerated vapor trap (RVT5105, Thermo Fisher Scientific). The residue was dissolved in $1 \mathrm{ml} 1 \mathrm{M}$ formic acid. Undissolved particles were pelleted before loading the samples on MCX columns (Oasis ${ }^{\circledR}$, Waters, $30 \mathrm{mg} 1 \mathrm{cc}$ ), which were previously equilibrated with $1 \mathrm{ml}$ 100\% methanol (UPC/ $\mathrm{MS}$ grade) followed by $1 \mathrm{ml}$ of $1 \mathrm{M}$ formic acid. Columns were washed with $1 \mathrm{ml} 1 \mathrm{M}$ formic acid and eluted with $1 \mathrm{ml}$ methanol. Samples were dried in a speedvac and stored dry at $4^{\circ} \mathrm{C}$. Samples where re-suspended in $100 \mu \mathrm{l} 50 \%$ acetonitrile containing $1 \%$ formic acid and filtered through a $0.45 \mu \mathrm{m}$ Minisart SRP4 filter (Sartorius, Germany) prior to analysis.

\section{Hormone Quantification by Liquid Chromatography-Tandem Mass Spectrometry (UPLC- MS/MS)}

Hormone quantification was performed on a Waters TQS Xevo tandem quadropole mass spectrometer equipped with an electrospray ionization source and coupled to an Acquity UPLC system (Waters, USA). Chromatographic separation was achieved using an Acquity 
UPLC BEH C18 column (100 x $2.1 \mathrm{~mm}, 1.7 \mu \mathrm{m})$ (Waters, USA) applying a 0.1\% formic acid / acetonitrile gradient, starting at $15 \%$ acetonitrile for $3.0 \mathrm{~min}$ and subsequently increasing to $50 \%(\mathrm{v} / \mathrm{v})$ acetonitrile in $16 \mathrm{~min}$. After that the column was rinsed which $100 \%$ acetonitrile for $2 \mathrm{~min}$, before equilibrating the column with $15 \%$ acetonitrile for $1 \mathrm{~min}$ before subsequent injection. Total run time was $20 \mathrm{~min}$. The column was operated at $40^{\circ} \mathrm{C}$ with a flow-rate of $0.4 \mathrm{ml} / \mathrm{min}$ and sample injection volume was $5 \mu \mathrm{l}$.

The mass spectrometer was operated in positive electrospray ionization (ESI) mode for OPDA, JA-Ile and IAA. SA, JA and ABA measurements where performed in negative electrospray ionization mode. The cone and desolvation gas flows were 150 and $800 \mathrm{~L} \mathrm{~h}^{-1}$, respectively. Argon was used for fragmentation by collision-induced dissociation in the ScanWave collision cell. The capillary voltage was set at $3 \mathrm{kV}$ in positive mode and $1 \mathrm{kV}$ in negative mode. The source temperature at $150^{\circ} \mathrm{C}$ and the desolvation gas temperature at $550^{\circ} \mathrm{C}$. Cone voltage (CV), collision energy (CE) and parent and daughter transitions were optimized for each component. Multiple reaction monitoring (MRM) transitions were selected based on the most abundant and specific fragment ions. The optimized parameters for OPDA, JA, JA-Ile, SA, ABA and IAA can be found in Supplemental Table 3.

Quantification was performed using a calibration curve with known amount of standards and based on the ratio of the peak areas of the MRM transition for standards to the MRM transition for the corresponding deuterium-labelled internal standards (Supplemental Table 10 numbers 1-7). Data acquisition and analysis were performed using MassLynx 4.1 (TargetLynx) software (Waters, USA).

\section{CRISPR/Cas9 Mutagenesis and Histology}

Stable transformation and CRISPR/Cas9 mutagenesis is done as described in Chapter 5. Single-guide RNAs (sgRNAs) were PCR amplified using primers listed in Supplemental Table 4. Three sgRNAs were designed for each gene using the CRISPR design tool implemented in Geneious R9 (Biomatters, Auckland, New Zealand). For double knockouts, six sgRNAs were expressed using a single binary construct. Genotyping of candidate mutants was performed using gene-specific primers listed in Supplemental Table 4.

Nodule tissue was fixed in $5 \%$ glutaraldehyde PBS $(\mathrm{pH}=7.2)$ solution overnight. After fixation, samples were dehydrated in an ethanol series and subsequently embedded in Technovit 7100 (Heraeus-Kulzer, Wehrheim, Germany), according to the manufacturer's protocol. Semi-thin $(3.5 \mu \mathrm{m})$ sections were cut using a microtome (Reichert-Jung, Leica 
Microsystems, Rijswijk, The Netherlands) and stained with $0.05 \%$ Toluidine Blue-O, before being photographed using a Leica DM5500B microscope equipped with a DFC425C camera (Leica Microsystems, Wetzlar, Germany).

\section{Accession Numbers}

GenelDs of all genes mentioned in this chapter can be found in Supplemental Table 5. Sequences can be downloaded from www.parasponia.org.

\section{Author Contributions}

R.G. conceived of and supervised the study together with T.B.; A.v.Z. designed and planned experiments, with input from R.G., R.v.V. and W.K.; A.v.Z., R.v.V., M.H., S.L. and W.K. prepared samples for RNA isolation and hormone measurements; A.v.Z. and J.V. isolated RNA and R.v.V. and R.H. performed RNA-seq read mapping; A.v.Z. performed differential expression analysis; K.G. performed hormone quantification; A.v.Z., M.H. and S.L. generated CRISPR/ Cas9 mutants and determined mutant phenotypes; A.v.Z. and R.G. analyzed the data; A.v.Z. wrote the text and R.G. assisted in editing the chapter.

\section{ACKNOWLEDGEMENTS}

The authors like to thank Luuk Rutten, Titis Wardhani, Susan Moenga, Max van Hooren, Fleur Brummans, Maikel Steentjes, Roos Goessen and Matthijs Oosterbeek for their contributions to the project, as part of their MSc. or BSc. training. The authors are grateful to Yuda Roswanjaya for sharing results prior to publication. This work was supported by NWO-NSFC Joint Research project (846.11.005) to R.G. and T.B., NWO-VICl (865.13.001) to R.G., NWO-VENI (863.15.010) to W.K. and the European Research Council (ERC-2011AdG294790) to T.B.

\section{REFERENCES}

1 Delaux, P.M. et al. (2015) Tracing the evolutionary path to nitrogen-fixing crops. Curr. Opin. Plant Biol. 26, 95-99

2 Geurts, R. et al. (2016) What does it take to evolve a nitrogen-fixing endosymbiosis? Trends Plant Sci. 21, 199-208

3 Doyle, J.J. (2011) Phylogenetic perspectives on the origins of nodulation. Mol. Plant. Microbe Interact. 24, 1289-1295

4 Soltis, D.E. et al. (1995) Chloroplast gene sequence data suggest a single origin of the predisposition for symbiotic nitrogen-fixation in angiosperms. Proc. Natl. Acad. Sci. U. S. A. 92, 2647-2651

5 Werner, G.D.A. et al. (2014) A single evolutionary innovation drives the deep evolution of symbiotic N-2fixation in angiosperms. Nat. Commun. 5, 4087

6 Li, H.-L. et al. (2015) Large-scale phylogenetic analyses reveal multiple gains of actinorhizal nitrogen-fixing 
symbioses in angiosperms associated with climate change. Sci. Rep. 5, 14023

Akkermans, A.D.L. et al. (1978) Nitrogen-fixing root nodules in Ulmaceae. Nature 274, 190

8 Clason, E.W. (1936) The vegetation of the upper-Badak region of mount Kelut (east java). Bulletin Jard. Bot. Buitenzorg Serie III, 509-518

9 Trinick, M.J. (1973) Symbiosis between Rhizobium and the non-legume, Trema aspera. Nature 244, 459-460

10 Ane, J.M. et al. (2004) Medicago truncatula DMI1 required for bacterial and fungal symbioses in legumes. Science 303, 1364-1367

11 Groth, M. et al. (2010) NENA, a Lotus japonicus homolog of Sec13, is required for rhizodermal infection by arbuscular mycorrhiza fungi and rhizobia but dispensable for cortical endosymbiotic development. Plant Cell 22, 2509-2526

12 Horvath, B. et al. (2011) Medicago truncatula IPD3 is a member of the common symbiotic signaling pathway required for rhizobial and mycorrhizal symbioses. Mol. Plant. Microbe Interact. 24, 1345-1358

13 Imaizumi-Anraku, H. et al. (2005) Plastid proteins crucial for symbiotic fungal and bacterial entry into plant roots. Nature 433, 527-531

14 Kanamori, N. et al. (2006) A nucleoporin is required for induction of $\mathrm{Ca}^{2+}$ spiking in legume nodule development and essential for rhizobial and fungal symbiosis. Proc. Natl. Acad. Sci. U. S. A. 103, 359-364

15 Levy, J. et al. (2004) A putative $\mathrm{Ca}^{2+}$ and calmodulin-dependent protein kinase required for bacterial and fungal symbioses. Science 303, 1361-1364

16 Saito, K. et al. (2007) NUCLEOPORIN85 is required for calcium spiking, fungal and bacterial symbioses, and seed production in Lotus japonicus. Plant Cell 19, 610-624

17 Stracke, S. et al. (2002) A plant receptor-like kinase required for both bacterial and fungal symbiosis. Nature $417,959-962$

18 Denarie, J. et al. (1996) Rhizobium lipo-chitooligosaccharide nodulation factors: Signaling molecules mediating recognition and morphogenesis. Annu. Rev. Biochem. 65, 503-535

19 Maillet, F. et al. (2011) Fungal lipochitooligosaccharide symbiotic signals in arbuscular mycorrhiza. Nature 469, 58-63

20 Arrighi, J.F. et al. (2006) The Medicago truncatula lysine motif-receptor-like kinase gene family includes NFP and new nodule-expressed genes. Plant Physiol. 142, 265-279

21 Limpens, E. et al. (2003) LysM domain receptor kinases regulating rhizobial Nod factor-induced infection. Science 302, 630-633

22 Madsen, E.B. et al. (2003) A receptor kinase gene of the LysM type is involved in legume perception of rhizobial signals. Nature 425, 637-640

23 Radutoiu, S. et al. (2003) Plant recognition of symbiotic bacteria requires two LysM receptor-like kinases. Nature 425, 585-592

24 Oldroyd, G.E.D. (2013) Speak, friend, and enter: signalling systems that promote beneficial symbiotic associations in plants. Nat. Rev. Microbiol. 11, 252-263

25 Timmers, A.C.J. et al. (1999) Refined analysis of early symbiotic steps of the Rhizobium-Medicago interaction in relationship with microtubular cytoskeleton rearrangements. Development 126, 3617-3628

26 Xiao, T.T. et al. (2014) Fate map of Medicago truncatula root nodules. Development 141, 3517-3528

27 Huisman, R. et al. (2016) A symbiosis-dedicated SYNTAXIN OF PLANTS 13II isoform controls the formation of a stable host-microbe interface in symbiosis. New Phytol. 211, 1338-1351

28 Ivanov, S. et al. (2012) Rhizobium-legume symbiosis shares an exocytotic pathway required for arbuscule formation. Proc. Natl. Acad. Sci. U. S. A. 109, 8316-8321

29 Pan, H.R. et al. (2016) A symbiotic SNARE protein generated by alternative termination of transcription. Nat. Plants 2, 15197

30 Limpens, E. et al. (2015) Lipochitooligosaccharides modulate plant host immunity to enable endosymbioses. Annu. Rev. Phytopathol. 53, 311-334

31 Zipfel, C. and Oldroyd, G.E.D. (2017) Plant signalling in symbiosis and immunity. Nature 543, 328-336

32 Blilou, I. et al. (1999) Resistance of pea roots to endomycorrhizal fungus or Rhizobium correlates with enhanced levels of endogenous salicylic acid. J. Exp. Bot. 50, 1663-1668

33 Martinez-Abarca, F. et al. (1998) Involvement of salicylic acid in the establishment of the Rhizobium meliloti - Alfalfa symbiosis. Mol. Plant. Microbe Interact. 11, 153-155

34 Caarls, L. et al. (2015) How salicylic acid takes transcriptional control over jasmonic acid signaling. Front. 
Plant Sci. 6, 170

35 Glazebrook, J. (2005) Contrasting mechanisms of defense against biotrophic and necrotrophic pathogens. Annu. Rev. Phytopathol. 43, 205-227

36 Thaler, J.S. et al. (2012) Evolution of jasmonate and salicylate signal crosstalk. Trends Plant Sci. 17, 260-270

37 Wasternack, C. and Hause, B. (2013) Jasmonates: biosynthesis, perception, signal transduction and action in plant stress response, growth and development. An update to the 2007 review in Annals of Botany. Ann. Bot. 111, 1021-1058

38 Fonseca, S. et al. (2009) (+)-7-iso-Jasmonoyl-L-isoleucine is the endogenous bioactive jasmonate. Nat. Chem. Biol. 5, 344-350

39 Bueno, P. et al. (2001) Time-course of lipoxygenase, antioxidant enzyme activities and $\mathrm{H}_{2} \mathrm{O}_{2}$ accumulation during the early stages of Rhizobium-legume symbiosis. New Phytol. 152, 91-96

40 Sun, J.H. et al. (2006) Crosstalk between jasmonic acid, ethylene and Nod factor signaling allows integration of diverse inputs for regulation of nodulation. Plant J. 46, 961-970

41 Suzuki, A. et al. (2011) Lotus japonicus nodulation is photomorphogenetically controlled by sensing the red/ far red (R/FR) ratio through jasmonic acid (JA) signaling. Proc. Natl. Acad. Sci. U. S. A. 108, 16837-16842

42 van Velzen, R. et al. (2017) Parallel loss of symbiosis genes in relatives of nitrogen-fixing non-legume Parasponia. bioRxiv DOI: 10.1101/169706

43 Yang, M.Q. et al. (2013) Molecular phylogenetics and character evolution of Cannabaceae. Taxon 62, 473-485

44 Becking, J.H. (1983) The Parasponia parviflora - rhizobium symbiosis - isotopic nitrogen-fixation, hydrogen evolution and nitrogen-fixation efficiency, and oxygen relations. Plant Soil 75, 343-360

45 Murray, J.D. et al. (2011) Vapyrin, a gene essential for intracellular progression of arbuscular mycorrhizal symbiosis, is also essential for infection by rhizobia in the nodule symbiosis of Medicago truncatula. Plant $J$. 65, 244-252

46 Pumplin, N. et al. (2010) Medicago truncatula Vapyrin is a novel protein required for arbuscular mycorrhizal symbiosis. Plant J. 61, 482-494

47 Javot, H. et al. (2007) A Medicago truncatula phosphate transporter indispensable for the arbuscular mycorrhizal symbiosis. Proc. Natl. Acad. Sci. U. S. A. 104, 1720-1725

48 Xue, L. et al. (2015) Network of GRAS transcription factors involved in the control of arbuscule development in Lotus japonicus. Plant Physiol. 167, 854-871

49 Zhang, Q. et al. (2010) Two Medicago truncatula half-ABC transporters are essential for arbuscule development in arbuscular mycorrhizal symbiosis. Plant Cell 22, 1483-1497

50 Gobbato, E. et al. (2012) A GRAS-type transcription factor with a specific function in mycorrhizal signaling. Curr. Biol. 22, 2236-2241

51 Pimprikar, P. et al. (2016) A CCaMK-CYCLOPS-DELLA complex activates transcription of RAM1 to regulate arbuscule branching. Curr. Biol. 26, 987-998

52 Takeda, N. et al. (2009) Apoplastic plant subtilases support arbuscular mycorrhiza development in Lotus japonicus. Plant J. 58, 766-777

53 Wang, E.T. et al. (2012) A common signaling process that promotes mycorrhizal and oomycete colonization of plants. Curr. Biol. 22, 2242-2246

54 Hohnjec, N. et al. (2005) Overlaps in the transcriptional profiles of Medicago truncatula roots inoculated with two different Glomus fungi provide insights into the genetic program activated during arbuscular mycorrhiza. Plant Physiol. 137, 1283-1301

55 Guether, M. et al. (2009) A mycorrhizal-specific ammonium transporter from Lotus japonicus acquires nitrogen released by arbuscular mycorrhizal fungi. Plant Physiol. 150, 73-83

56 Nemhauser, J.L. et al. (2006) Different plant hormones regulate similar processes through largely nonoverlapping transcriptional responses. Cell 126, 467-475

57 Floss, D.S. et al. (2013) DELLA proteins regulate arbuscule formation in arbuscular mycorrhizal symbiosis. Proc. Natl. Acad. Sci. U. S. A. 110, E5025-E5034

58 Bell, E. et al. (1995) A chloroplast lipoxygenase is required for wound-induced jasmonic acid accumulation in Arabidopsis. Proc. Natl. Acad. Sci. U. S. A. 92, 8675-8679

59 Mine, A. et al. (2017) An incoherent feed-forward loop mediates robustness and tunability in a plant immune network. EMBO Rep. 18, 464-476

60 Reymond, P. et al. (2004) A conserved transcript pattern in response to a specialist and a generalist herbivore. 
Plant Cell 16, 3132-3147

61 Sasaki, Y. et al. (2001) Monitoring of methyl jasmonate-responsive genes in Arabidopsis by cDNA macroarray: Self-activation of jasmonic acid biosynthesis and crosstalk with other phytohormone signaling pathways. DNA Res. 8, 153-161

62 Stacey, G. et al. (2006) Effects of endogenous salicylic acid on nodulation in the model legumes Lotus japonicus and Medicago truncatula. Plant Physiol. 141, 1473-1481

63 Hayashi, S. et al. (2008) Molecular analysis of lipoxygenases associated with nodule development in soybean. Mol. Plant. Microbe Interact. 21, 843-853

64 Zdyb, A. et al. (2011) Jasmonate biosynthesis in legume and actinorhizal nodules. New Phytol. 189, 568-579

65 Floss, D.S. et al. (2016) DELLA proteins regulate expression of a subset of AM symbiosis-induced genes in Medicago truncatula. Plant Signal. Behav. 11, e1162369

66 Park, H.J. et al. (2015) Hyphal branching during arbuscule development requires Reduced Arbuscular Mycorrhiza1. Plant Physiol. 169, 2774-2788

67 Jiang, C.F. et al. (2007) Phosphate starvation root architecture and anthocyanin accumulation responses are modulated by the gibberellin-DELLA signaling pathway in Arabidopsis. Plant Physiol. 145, 1460-1470

68 Khan, G.A. et al. (2016) Phosphate deficiency induces the jasmonate pathway and enhances resistance to insect herbivory. Plant Physiol. 171, 632-644

69 Castrillo, G. et al. (2017) Root microbiota drive direct integration of phosphate stress and immunity. Nature 543, 513-518

70 Vazquez-Yanes, C. (1998) Trema micrantha (L.) Blume (Ulmaceae): A promising neotropical tree for site amelioration of deforested land. Agrofor. Syst. 40, 97-104

71 Elias, T.S.C. (1970) The genera of Ulmaceae in the southeastern United States. Journal of the Arnold Arboretum 51, 18-40

72 Soepadmo, E. (1974) Ulmaceae. Flora Malesiana-Series 1, Spermatophyta 8, 31-76

73 Becking, J.H. (1992) The Rhizobium symbiosis of the nonlegume Parasponia. In Biological nitrogen fixation, pp. 497-559, Routledge, Chapman and Hall

74 Akkermans, A.D.L. et al. (1978) $\mathrm{N}_{2}$-fixing root nodules in Ulmaceae: Parasponia or (and) Trema spp.? Plant Soil 49, 711-715

75 Trinick, M.J. (1980) Growth of Parasponia in agar tube culture and symbiotic effectiveness of isolates from Parasponia spp. New Phytol. 85, 37-45

76 Carbonnel, S. and Gutjahr, C. (2014) Control of arbuscular mycorrhiza development by nutrient signals. Front. Plant Sci. 5, 462

77 Lebeis, S.L. et al. (2015) Salicylic acid modulates colonization of the root microbiome by specific bacterial taxa. Science 349, 860-864

78 Zgadzaj, R. et al. (2016) Root nodule symbiosis in Lotus japonicus drives the establishment of distinctive rhizosphere, root, and nodule bacterial communities. Proc. Natl. Acad. Sci. U. S. A. 113, E7996-E8005

79 Bender, G.L. and Rolfe, B.G. (1985) A rapid plant assay for the Parasponia-rhizobium symbiosis. Plant Sci. 38, 135-140

80 Cao, Q. et al. (2012) Efficiency of Agrobacterium rhizogenes-mediated root transformation of Parasponia and Trema is temperature dependent. Plant Growth Regul. 68, 459-465

81 Kim, D. et al. (2015) HISAT: a fast spliced aligner with low memory requirements. Nat. Methods 12, 357-360

82 Liao, Y. et al. (2014) featureCounts: an efficient general purpose program for assigning sequence reads to genomic features. Bioinformatics 30, 923-930

83 Love, M.I. et al. (2014) Moderated estimation of fold change and dispersion for RNA-seq data with DESeq2. Genome Biol. 15, 550

84 Dobrev, P.I. and Kaminek, M. (2002) Fast and efficient separation of cytokinins from auxin and abscisic acid and their purification using mixed-mode solid-phase extraction. J. Chromatogr. A 950, 21-29 


\section{SUPPLEMENTAL DATA}

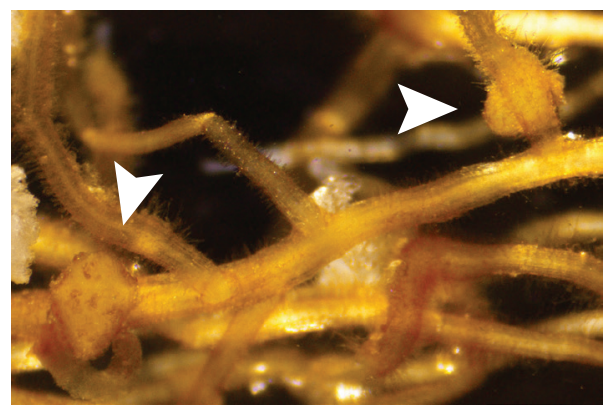

Supplemental Figure 1. Experimental conditions are conducive for Parasponia root nodule formation. Root nodules are formed on roots of $P$. rigida grown from seed in perlite. Perlite was watered with EKM $\left(5 \mathrm{mM} \mathrm{KNO}_{3}\right)$ and inoculated with Mesorhizobium plurifarium BOR2. Arrowheads indicate nodules.

Supplemental Figure 2. Principal component analysis is not affected by mapping to different genomes.

Principal component analysis (PCA) on gene expression values in roots of two Parasponia and three Trema species.

(A) PCA was performed on DESeq2 'regularized log' transformed read counts obtained after mapping of $P$. andersonii and $P$. rigida reads to the $P$. andersonii genome and $T$. levigata, $T$. orientalis and $T$. tomentosa reads against the $T$. orientalis genome. Note: this plot is identical to the plot shown in Fig. 1. (B) PCA was performed on DESeq2 'regularized log' transformed read counts obtained after mapping of reads from all five species to the $P$. andersonii genome.

(C) PCA was performed on DESeq2 'regularized log' transformed read counts obtained after mapping reads from all five species to the $T$. orientalis genome.

Plotted are first two principal components (PC1 and PC2) and indicated is the percentage of explained variance by each principal component. 
A

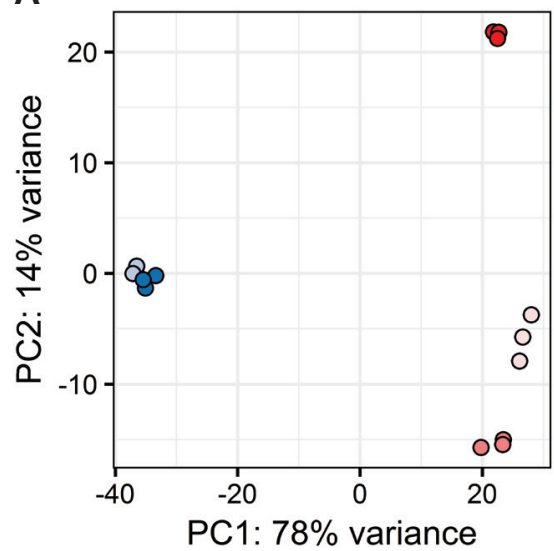

O P. andersonii

- P. rigida

- T. levigata

- T. orientalis

- T. tomentosa

B

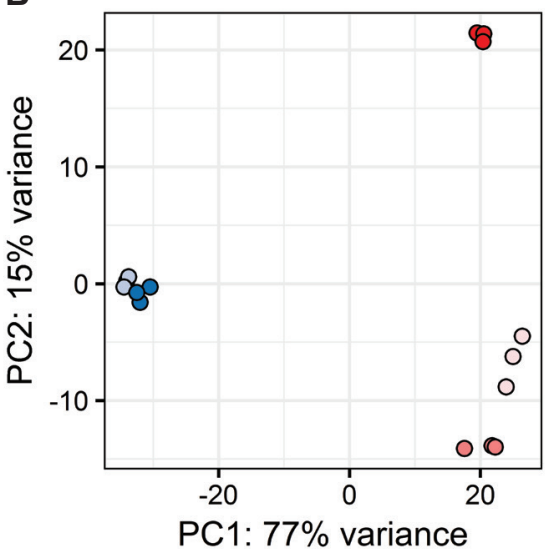

O P. andersonii

- $P$. rigida

O T. levigata

- T. orientalis

- T. tomentosa

C

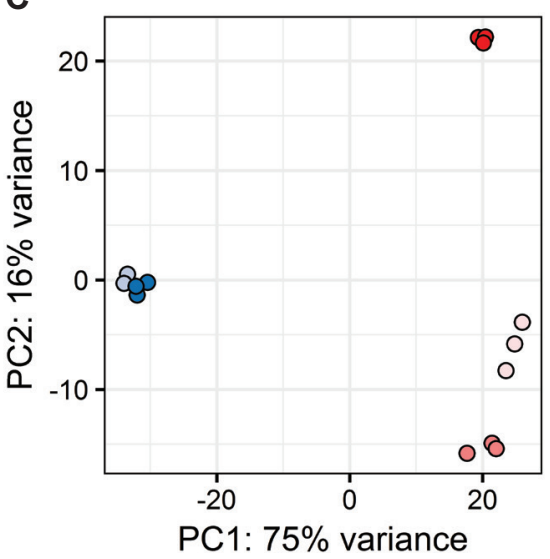

O P. andersonii

- P. rigida

- T. levigata

- T. orientalis

- T. tomentosa 


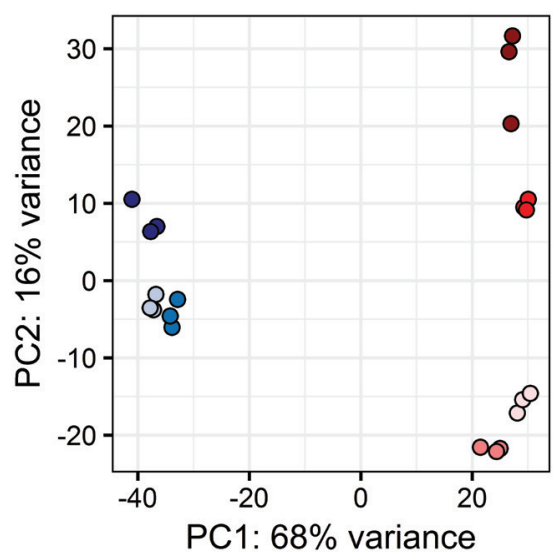

\begin{tabular}{c}
$\begin{array}{c}\text { Tissue culture } \\
\text { plantlets }\end{array}$ \\
\hline o P. andersonii \\
- T. tomentosa \\
Plants grown \\
from seed \\
\hline o P. andersonii \\
o P. rigida \\
O T. levigata \\
O T. orientalis \\
- T. tomentosa
\end{tabular}

Supplemental Figure 3. Principal component analysis including expression values from plants obtained through in vitro micropropagation.

Principal component analysis (PCA) on gene expression values in roots of two Parasponia and three Trema species grown from seed and roots of $P$. andersonii and $T$. tomentosa plants obtained through in vitro micro-propagation. PCA was performed on DESeq2 'regularized log' transformed read counts obtained after mapping of $P$. andersonii and $P$. rigida reads to

the $P$. andersonii genome and $T$. levigata, $T$. orientalis and $T$. tomentosa reads against the $T$. orientalis genome. Plotted are first two principal components (PC1 and PC2) and indicated is the percentage of explained variance by each principal component.
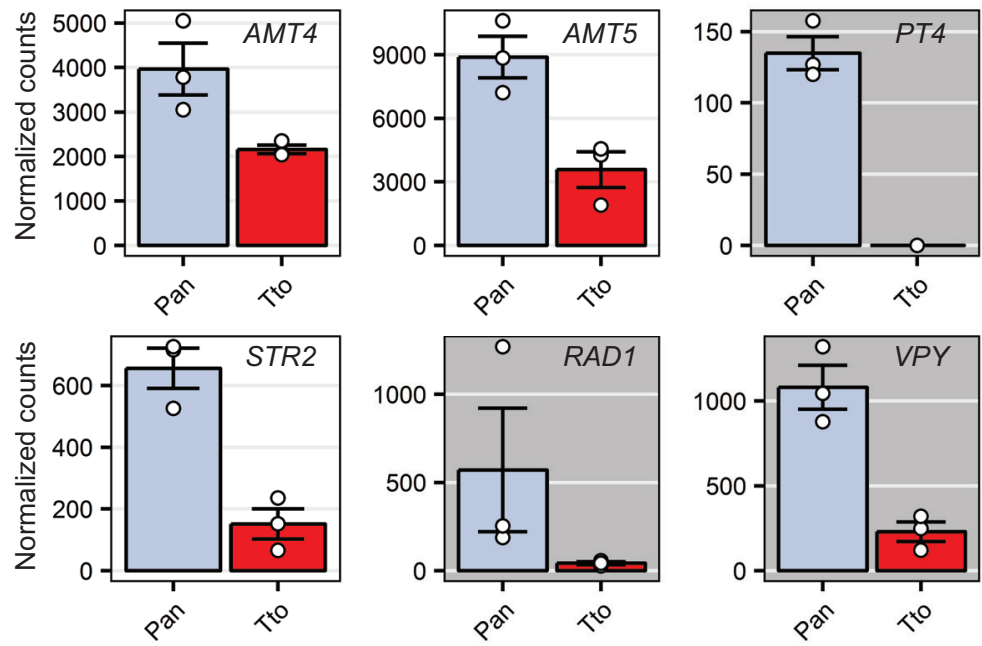

Supplemental Figure 4. Expression of mycorrhizal marker genes in roots of $P$. andersonii and $T$. tomentosa plants obtained through in vitro micro-propagation.

Expression of the mycorrhizal marker genes AMT4, AMT5, PT4, STR2, RAD1 and VPY in roots of $P$. andersonii (Pan) and T. tomentosa (Tto) plants obtained through in vitro micro-propagation. Expression levels were calculated after mapping of $P$. andersonii reads to the $P$. andersonii genome and $T$. tomentosa reads against the $T$. orientalis genome. Expression levels are presented as DESeq2 normalized read counts. Data represent means of 3 biological replicates \pm SEM. Dots represent expression values in each of the biological replicates. Grey panel highlighting of expression plots indicates differential expression (fold change $>2, p<0.05$ ), consistent across all comparisons. 

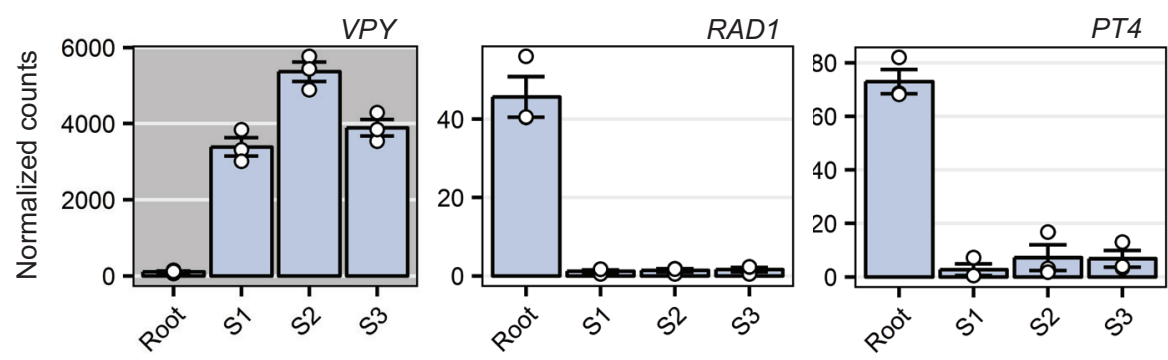

Supplemental Figure 5. Expression of VPY, RAD1 and PT4 in P. andersonii roots and nodules.

Expression of PanVPY, PanRAD1 and PanPT4 in roots and during three stages of nodule development (S1-S3). Expression levels are presented as DESeq2 normalized read counts. Data represent means of 3 biological replicates \pm SEM. Dots represent expression values in each of the biological replicates. Grey panel highlighting indicates significant nodule-enhanced expression (fold change $>2, p<0.05$ ), according to van Velzen et al. [42].
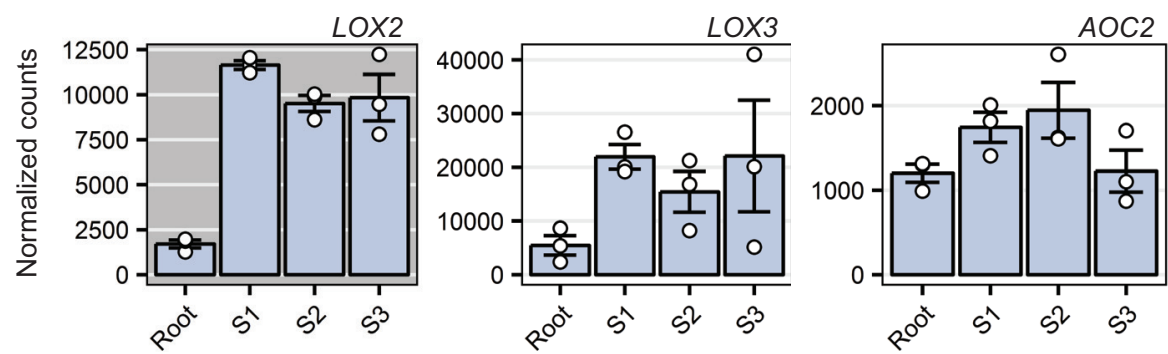

Supplemental Figure 6. Expression of LOX2, LOX3 and AOC2 in P. andersonii roots and nodules.

Expression of PanLOX2, PanLOX3 and PanAOC2 in roots and during three stages of nodule development (S1-S3). Expression levels are presented as DESeq2 normalized read counts. Data represent means of 3 biological replicates \pm SEM. Dots represent expression values in each of the biological replicates. Grey panel highlighting indicates significant nodule-enhanced expression (fold change $>2, p<0.05$ ), according to van Velzen et al. [42]. 
A

$1000 \mathrm{bp}$

B

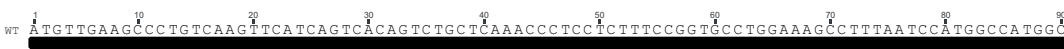

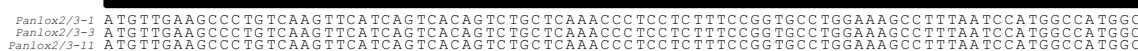

AGCAATCTTĞ

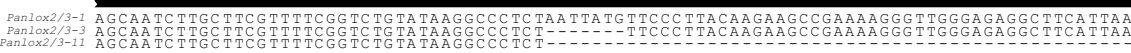

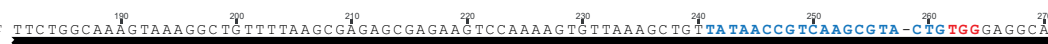

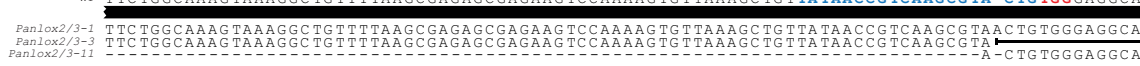

WT TITTGACAAACCTTAATTTAC-AGCGAGGACTCGATGATA T TCAAGATT 300

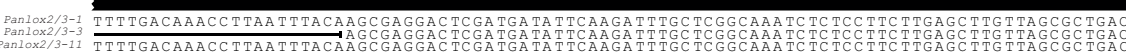

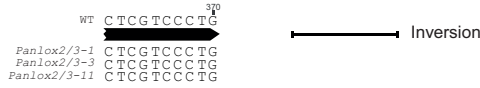

C

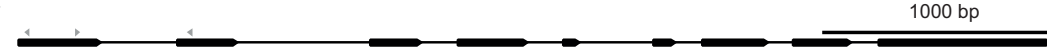

D

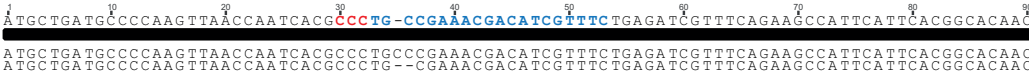

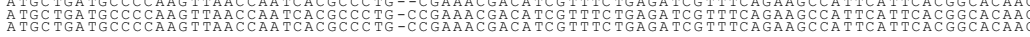

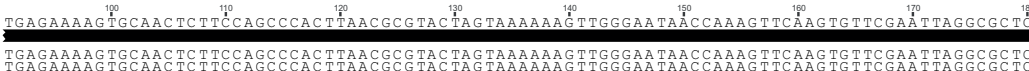

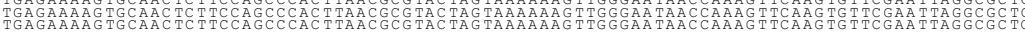

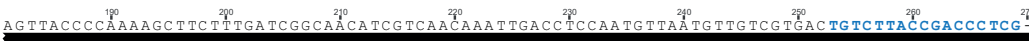
AGTTACCCCAAAAG TTCTTTGATCG GCAACA TCG TCAACAATTGACC TCCAATG TTAATGTTG TCG TGACTGTCT TACCGACCC TCGA

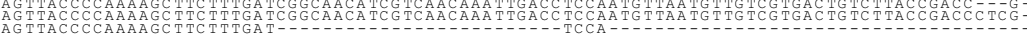
GAGGG TTT ThACAAACG 200 GAGGGGTPTTGACAAACGTTGG TATAGGAAAAGGAATTGACGATGCAAAAGATTTGCTCGGCCAATCACTCCAACTTGAGCTCGTCAGTG

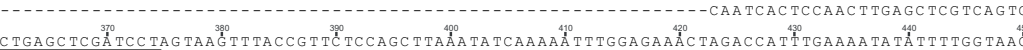

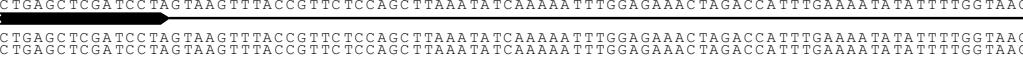
CTGAGCTCGATCCTAGTAAGTTACCGTTCTCAGCTTAAATATCAAAAATTTGGAGAAACTAGACATTTGAAAATATATT TTGGTAAC

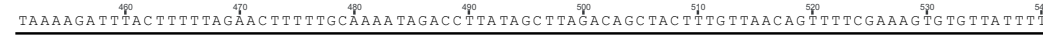

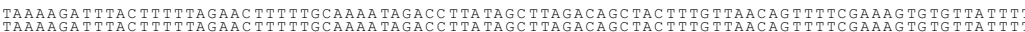
TAAAAGATTACTTTTAGAACTTTTGCAAAATAGACCTTATAGCTTAGACAGCTACTTGTTAACAGTTTCGAAAGTGTGTATT

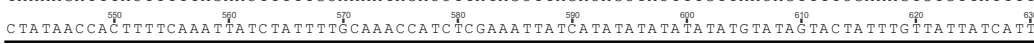

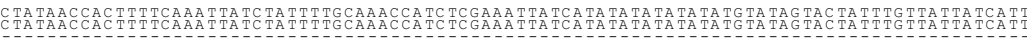

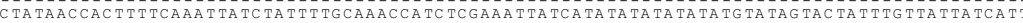

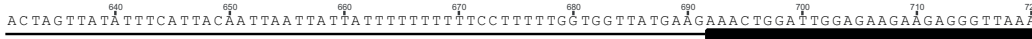

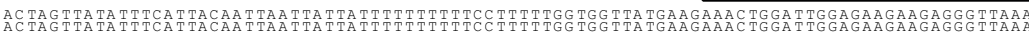
ACTAGTTATATTCATTACAATTAATARATTTTTTTTCCTTTTGGGGTATGAAGAACTGGATTGGAGAAGAAGAGGGTAA

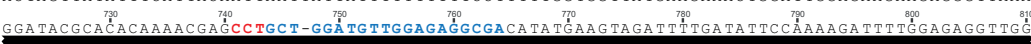
GGATACGCACACAAAACGAGCCTGCTGGATGTTGGAGAGGCGACATATGAAGTAGATTTGATATTCCAAAAGATTTTGGAGAGGTTGG GGATACGCACACAAAACGAGCCTGC-DGGGTTGGAGGGCGACATATGAAGTAGATTTTGATATTCCAAAAGATTTTGGAGAGGTTGE

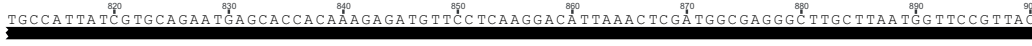
TGCCATTATCGTGCAGAATGAGCACCACAAAGAGATG TTCC TCAAGGACATPAAACTCGATGGCGAGGCTTGCTTAATGGTTCCGTPAC TGGCATPATCGTGCAGAATGAGCACCACAAAGAGATGTTCCTCAAGGACATTAAACTCGATGGCGAGGCTPGCTTAATGGTTCCGTTAC

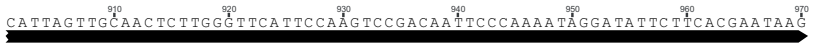

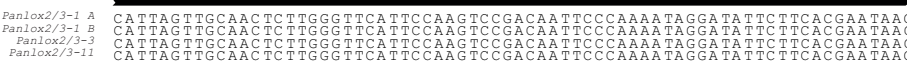




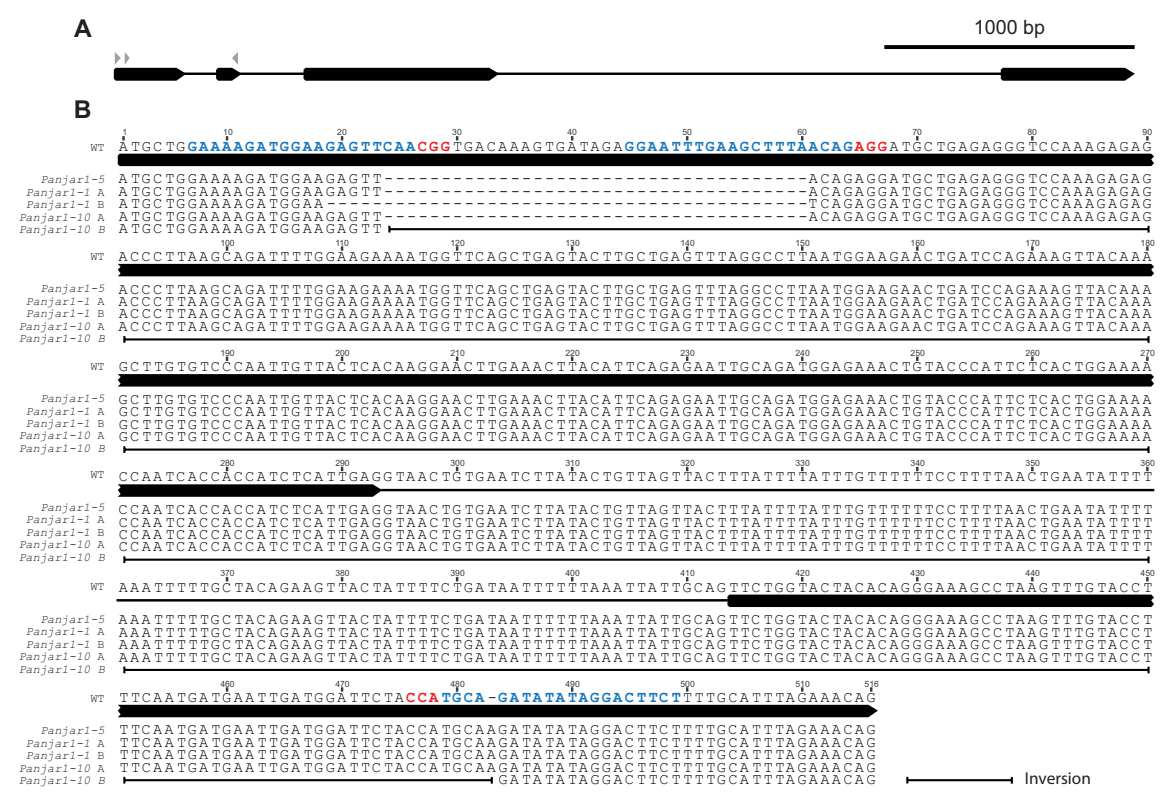

Supplemental Figure 8. CRISPR mutant alleles of Panjar1 mutants.

(A) Schematic representation of PanJAR1 gene model, indicated are the locations of three sgRNA target sites.

(B) Alignment of PanJAR1 sequences in wild type (WT) and Panaoc1-Panaoc2 double mutant lines. Indicated in blue and red highlighting are the SgRNA target sites and PAM sequences, respectively.

Supplemental Figure 7. CRISPR mutant alleles of Panlox2-Panlox3 double mutants.

(A) Schematic representation of PanLOX2 gene model, indicated are the locations of three sgRNA target sites.

(B) Alignment of PanLOX2 sequences in wild type (WT) and Panaoc1-Panaoc2 double mutant lines. Indicated in blue and red highlighting are the sgRNA target site and PAM sequences, respectively.

(C) Schematic representation of PanLOX3 gene model, indicated are the locations of three sgRNA target sites.

(D) Alignment of PanLOX3 sequences in wild type (WT) and Panaoc1-Panaoc2 double mutant lines. Indicated in blue and red highlighting are the sgRNA target site and PAM sequences, respectively. 
A

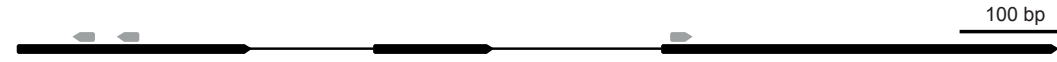

B

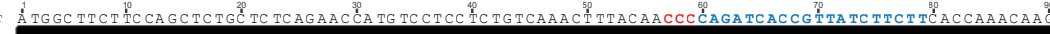

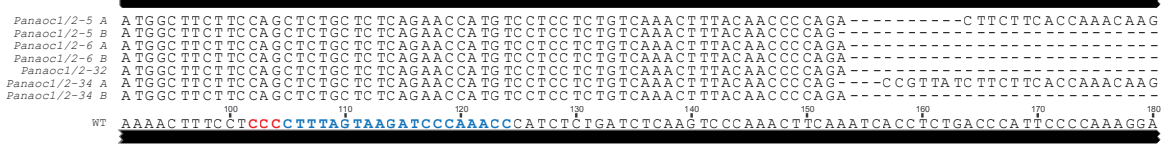

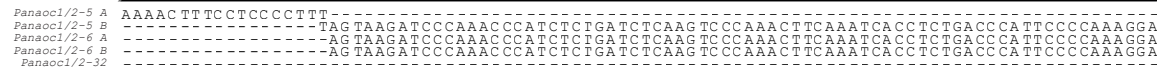

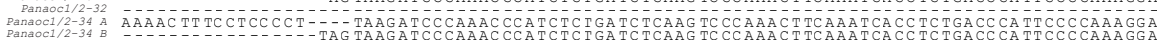
TCCTTCTTTTICCAAAAGCCAAAGTCAGGCCTATTCGTCAGAATCTGAAAGACCCCCTAG

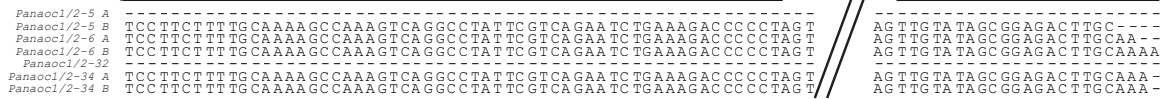
AA-GGATTGGTATAACACG

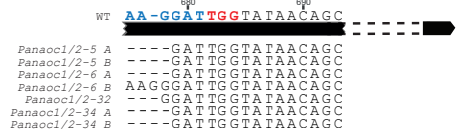

C

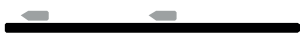

$100 \mathrm{bp}$

D

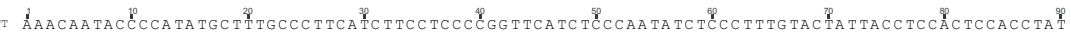

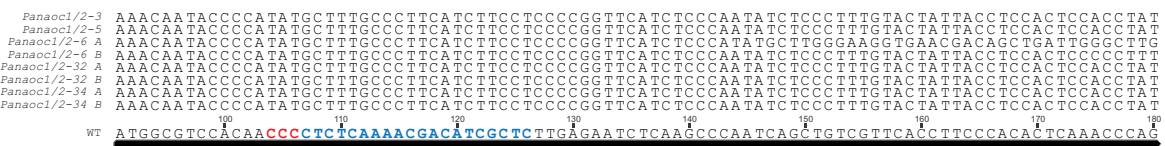

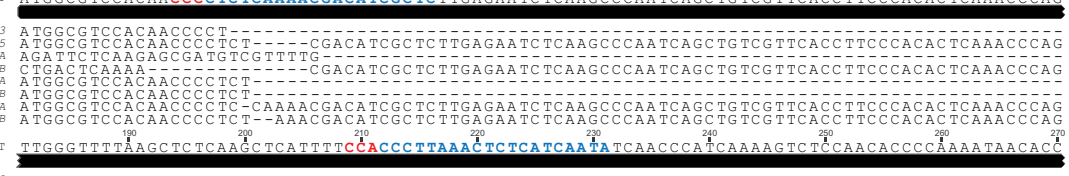

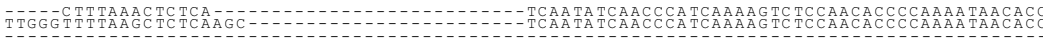
TIGGGTTTAAGCTCTCAAGCTCATTTTCCACCC

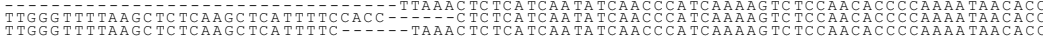

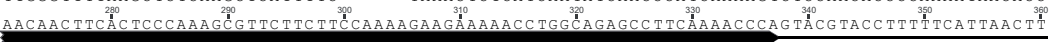

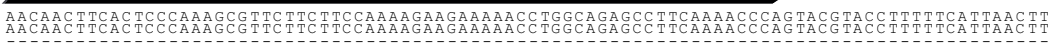

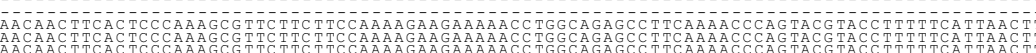

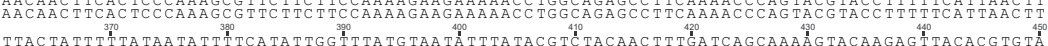

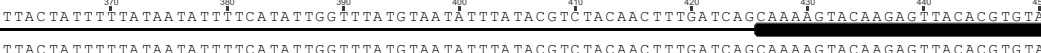

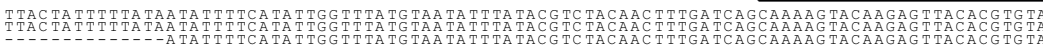

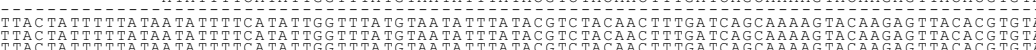

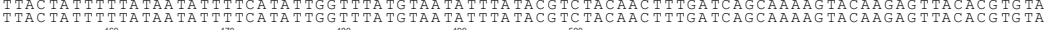

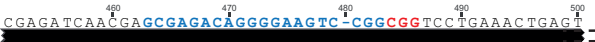



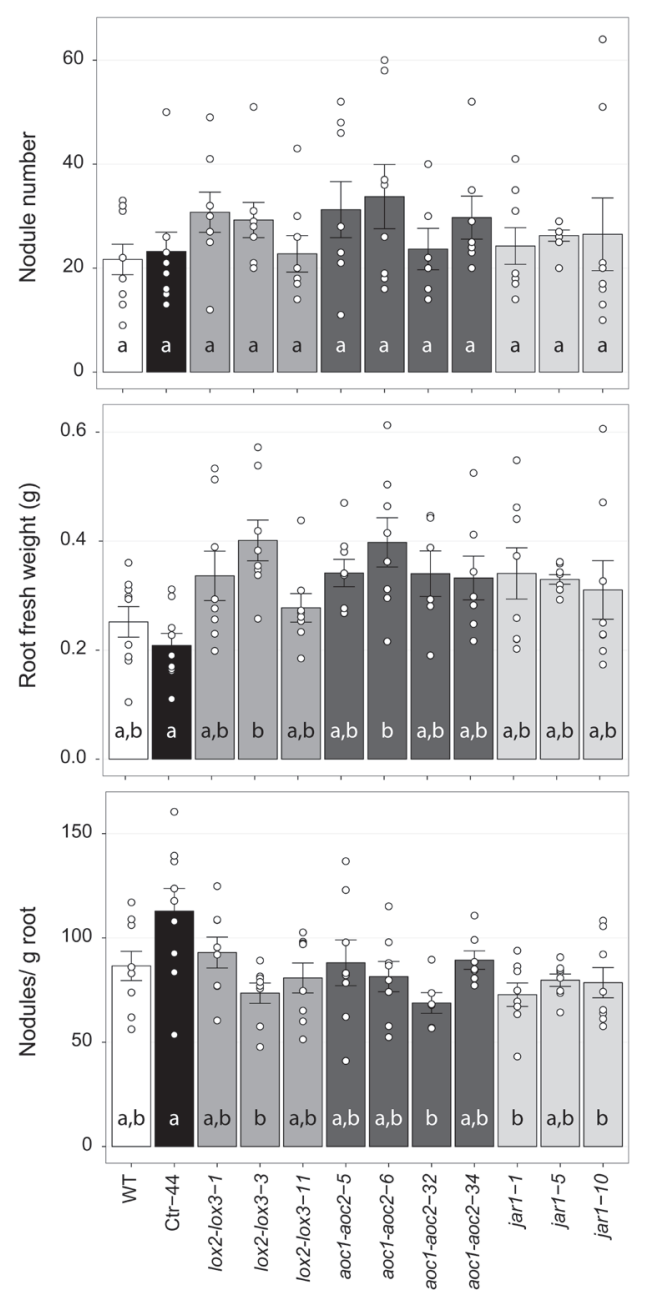

Supplemental Figure 10. Nodulation data of JA mutants.

Nodule formation on JA biosynthesis mutants at 6 weeks after inoculation with $M$. plurifarium BOR2. Data represent means of 6-9 biological replicates \pm SEM. Dots represent individual data points. Different letters on bars indicate statistical significance ( $p<0.05$, ANOVA with Tukey post-hoc test). This data corresponds to the data shown in Figure 5. WT, wild-type P. andersonii; Ctr-44, transgenic control line expressing the Cas9 gene only [Chapter 5].

Supplemental Figure 9. CRISPR mutant alleles of Panaoc1-Panaoc2 double mutants.

(A) Schematic representation of PanAOC1 gene model, indicated are the locations of three sgRNA target sites.

(B) Alignment of PanAOC1 sequences in wild type (WT) and Panaoc1-Panaoc2 double mutant lines. Indicated in blue and red highlighting are the sgRNA target site and PAM sequences, respectively. Shown are the first exon and beginning of the third exon.

(C) Schematic representation of PanAOC2 gene model, indicated are the locations of three sgRNA target sites.

(D) Alignment of PanAOC2 sequences in wild type (WT) and Panaoc1-Panaoc2 double mutant lines. Indicated in blue and red highlighting are the sgRNA target site and PAM sequences, respectively. Shown are exon 1 and the beginning of exon 2 . 
Supplemental Table 1. Genes consistently higher expressed in roots of Parasponia compared to Trema.

\begin{tabular}{|c|c|c|c|}
\hline GenelD & Gene name & Functional annotation & $\begin{array}{c}\text { Nodule } \\
\text { enhanced }\end{array}$ \\
\hline PanWU01x14_asm01_ann01_147580 & PanBHLH2 & Basic helix-loop-helix transcription factor & TRUE \\
\hline PanWU01x14_asm01_ann01_284910 & PanCRK1 & Cysteine rich receptor like kinase & TRUE \\
\hline PanWU01x14_asm01_ann01_085160 & PanLOX2 & Lipoxygenase & TRUE \\
\hline PanWU01x14_asm01_ann01_341680 & PanVPY & Ankyrin repeat containing protein & TRUE \\
\hline PanWU01x14_asm01_ann01_227750 & - & Patatin-related protein & TRUE \\
\hline PanWU01x14_asm01_ann01_282200 & - & Acid phosphatase & TRUE \\
\hline PanWU01x14_asm01_ann01_065850 & - & Dienelactone hydrolase & TRUE \\
\hline PanWU01x14_asm01_ann01_223330 & - & Glycoside hydrolase & TRUE \\
\hline PanWU01x14_asm01_ann01_027160 & - & Bet v I/Major latex protein & TRUE \\
\hline PanWU01x14_asm01_ann01_272840 & - & Tyrosine-protein kinase & TRUE \\
\hline PanWU01x14_asm01_ann01_304770 & - & Serine/threonine protein kinase & TRUE \\
\hline PanWU01x14_asm01_ann01_255510 & - & Laccase & TRUE \\
\hline PanWU01x14_asm01_ann01_190770 & - & Cysteine-rich secretory protein, allergen V5/Tpx-1-related & TRUE \\
\hline PanWU01x14_asm01_ann01_072810 & - & Spastin & TRUE \\
\hline PanWU01x14_asm01_ann01_255030 & - & Germin & TRUE \\
\hline PanWU01x14_asm01_ann01_292340 & - & NB-ARC domain, LRR domain containing protein & TRUE \\
\hline PanWU01x14_asm01_ann01_011890 & - & Succinylglutamate-semialdehyde dehydrogenase & TRUE \\
\hline PanWU01x14_asm01_ann01_274070 & - & ATP-binding cassette containing protein & TRUE \\
\hline PanWU01x14_asm01_ann01_158610 & PanAOC2 & Allene oxide cyclase & FALSE \\
\hline PanWU01x14_asm01_ann01_262200 & PanBHLH85 & Basic helix-loop-helix transcription factor & FALSE \\
\hline PanWU01x14_asm01_ann01_364320 & PanCRK20 & Cysteine rich receptor like kinase & FALSE \\
\hline PanWU01x14_asm01_ann01_085170 & PanLOX3 & Lipoxygenase & FALSE \\
\hline PanWU01x14_asm01_ann01_197540 & PanMES7 & Methyl esterase & FALSE \\
\hline PanWU01x14_asm01_ann01_215630 & PanMYB71 & MYB transcription factor & FALSE \\
\hline PanWU01x14_asm01_ann01_171710 & PanNFYB10 & Nuclear transcription factor $Y$ subunit $B$ & FALSE \\
\hline PanWU01x14_asm01_ann01_341930 & PanNPF27 & Proton-dependent oligopeptide transporter & FALSE \\
\hline PanWU01x14_asm01_ann01_280620 & PanPAL3 & Phenylalanine ammonia-lyase & FALSE \\
\hline PanWU01x14_asm01_ann01_258770 & PanPIN5 & Auxin efflux carrier & FALSE \\
\hline PanWU01x14_asm01_ann01_225280 & PanPT4 & Phosphate transporter & FALSE \\
\hline PanWU01x14_asm01_ann01_257240 & PanRAD1 & GRAS transcription factor & FALSE \\
\hline PanWU01x14_asm01_ann01_134900 & PanTLP25 & Thaumatin & FALSE \\
\hline PanWU01x14_asm01_ann01_024300 & PanWAK9 & Wall-associated receptor kinase & FALSE \\
\hline PanWU01x14_asm01_ann01_073270 & PanWAT27 & Plant-drug/metabolite exporter & FALSE \\
\hline PanWU01x14_asm01_ann01_143830 & PanWRKY16 & WRKY domain containing protein & FALSE \\
\hline PanWU01x14_asm01_ann01_065890 & - & NB-ARC domain, LRR domain containing protein & FALSE \\
\hline PanWU01x14_asm01_ann01_072570 & - & Cytochrome P450, E-class, group I & FALSE \\
\hline PanWU01x14_asm01_ann01_202680 & - & Transferase & FALSE \\
\hline PanWU01x14_asm01_ann01_210240 & - & None & FALSE \\
\hline PanWU01x14_asm01_ann01_338770 & - & HAD-like domain containing protein & FALSE \\
\hline PanWU01x14_asm01_ann01_370590 & - & None & FALSE \\
\hline PanWU01x14_asm01_ann01_364540 & - & Major intrinsic protein & FALSE \\
\hline PanWU01x14_asm01_ann01_020190 & - & LRR domain containing protein & FALSE \\
\hline PanWU01x14_asm01_ann01_353370 & - & NB-ARC domain, LRR domain containing protein & FALSE \\
\hline PanWU01x14_asm01_ann01_018530 & - & Very-long-chain 3-ketoacyl-CoA synthase & FALSE \\
\hline PanWU01x14_asm01_ann01_156760 & - & Nucleoside phosphorylase & FALSE \\
\hline PanWU01x14_asm01_ann01_334870 & - & None & FALSE \\
\hline PanWU01x14_asm01_ann01_087710 & - & None & FALSE \\
\hline PanWU01x14_asm01_ann01_095210 & - & Oxoglutarate/iron-dependent dioxygenase & FALSE \\
\hline PanWU01x14_asm01_ann01_343310 & - & None & FALSE \\
\hline PanWU01x14_asm01_ann01_050940 & - & $\mathrm{NAD}(\mathrm{P})$-binding domain containing protein & FALSE \\
\hline PanWU01x14_asm01_ann01_015620 & - & None & FALSE \\
\hline PanWU01x14_asm01_ann01_249830 & - & Tyrosine-protein kinase & FALSE \\
\hline PanWU01x14_asm01_ann01_193420 & - & E3 ubiquitin-protein ligase SIN-like & FALSE \\
\hline PanWU01x14_asm01_ann01_320570 & - & Erythronate-4-phosphate dehydrogenase & FALSE \\
\hline PanWU01x14_asm01_ann01_170190 & - & Expansin & FALSE \\
\hline PanWU01x14_asm01_ann01_297550 & - & Serine/threonine protein kinase & FALSE \\
\hline PanWU01x14_asm01_ann01_044440 & - & DOMON domain containing protein & FALSE \\
\hline PanWU01x14_asm01_ann01_337840 & - & None & FALSE \\
\hline
\end{tabular}




\begin{tabular}{|c|c|c|c|}
\hline PanWU01x14_asm01_ann01_121030 & - & None & FALSE \\
\hline PanWU01x14_asm01_ann01_027420 & - & F-box domain containing protein & FALSE \\
\hline PanWU01x14_asm01_ann01_348670 & - & Organ specific protein & FALSE \\
\hline PanWU01x14_asm01_ann01_037440 & - & Transferase & FALSE \\
\hline PanWU01x14_asm01_ann01_153370 & - & None & FALSE \\
\hline PanWU01x14_asm01_ann01_221020 & - & None & FALSE \\
\hline PanWU01x14_asm01_ann01_059410 & - & None & FALSE \\
\hline PanWU01x14_asm01_ann01_072790 & - & Spastin & FALSE \\
\hline PanWU01x14_asm01_ann01_320590 & - & $\begin{array}{l}\text { D-isomer specific } 2 \text {-hydroxyacid dehydrogenase, } \\
\text { catalytic domain containing protein }\end{array}$ & FALSE \\
\hline PanWU01x14_asm01_ann01_086360 & - & GPCR kinase & FALSE \\
\hline PanWU01x14_asm01_ann01_199170 & - & UDP-glucuronosyl/UDP-glucosyltransferase & FALSE \\
\hline PanWU01x14_asm01_ann01_248980 & - & None & FALSE \\
\hline PanWU01x14_asm01_ann01_336310 & - & Transferase & FALSE \\
\hline PanWU01x14_asm01_ann01_221040 & - & None & FALSE \\
\hline PanWU01x14_asm01_ann01_037190 & - & NTF2-like domain containing protein & FALSE \\
\hline PanWU01x14_asm01_ann01_215550 & - & Glyoxalase I & FALSE \\
\hline PanWU01x14_asm01_ann01_365110 & - & None & FALSE \\
\hline PanWU01x14_asm01_ann01_006880 & - & None & FALSE \\
\hline PanWU01x14_asm01_ann01_165880 & - & Mlo-related protein & FALSE \\
\hline PanWU01x14_asm01_ann01_203150 & - & NADPH-dependent FMN reductase-like & FALSE \\
\hline PanWU01x14_asm01_ann01_257980 & - & Peroxidase & FALSE \\
\hline PanWU01x14_asm01_ann01_182570 & - & Pectinesterase inhibitor domain containing protein & FALSE \\
\hline PanWU01x14_asm01_ann01_022780 & - & None & FALSE \\
\hline PanWU01x14_asm01_ann01_000730 & - & S-receptor-like serine/threonine-protein kinase & FALSE \\
\hline PanWU01x14_asm01_ann01_097450 & - & None & FALSE \\
\hline PanWU01x14_asm01_ann01_028980 & - & Thylakoid lumenal $17.9 \mathrm{kDa}$ protein & FALSE \\
\hline PanWU01x14_asm01_ann01_049450 & - & Polyketide cyclase SnoaL-like domain containing protein & FALSE \\
\hline PanWU01x14_asm01_ann01_105700 & - & Very-long-chain 3-ketoacyl-CoA synthase & FALSE \\
\hline PanWU01x14_asm01_ann01_086300 & - & Seipin family & FALSE \\
\hline PanWU01x14_asm01_ann01_300310 & - & None & FALSE \\
\hline PanWU01x14_asm01_ann01_243090 & - & Transferase & FALSE \\
\hline PanWU01x14_asm01_ann01_338780 & - & B3 DNA binding domain containing protein & FALSE \\
\hline PanWU01x14_asm01_ann01_198170 & - & Photosystem II PsbO, manganese-stabilising & FALSE \\
\hline PanWU01x14_asm01_ann01_266880 & - & Octamer-binding transcription factor & FALSE \\
\hline PanWU01x14_asm01_ann01_271560 & - & None & FALSE \\
\hline PanWU01x14_asm01_ann01_298520 & - & Parvalbumin & FALSE \\
\hline PanWU01x14_asm01_ann01_032210 & - & None & FALSE \\
\hline PanWU01x14_asm01_ann01_266220 & - & 43kDa postsynaptic protein & FALSE \\
\hline PanWU01x14_asm01_ann01_339430 & - & Bifunctional P-protein, chorismate mutase/prephenate dehydratase & FALSE \\
\hline PanWU01x14_asm01_ann01_071410 & - & Gnk2-like domain containing protein & FALSE \\
\hline PanWU01x14_asm01_ann01_111660 & - & Cation transporter & FALSE \\
\hline PanWU01x14_asm01_ann01_011250 & - & None & FALSE \\
\hline PanWU01x14_asm01_ann01_059970 & - & Ionotropic glutamate receptor & FALSE \\
\hline PanWU01x14_asm01_ann01_216490 & - & None & FALSE \\
\hline PanWU01x14_asm01_ann01_199230 & - & UDP-glucuronosyl/UDP-glucosyltransferase & FALSE \\
\hline PanWU01x14_asm01_ann01_370000 & - & UDP-glucuronosyl/UDP-glucosyltransferase & FALSE \\
\hline PanWU01x14_asm01_ann01_148180 & - & Homeodomain-like & FALSE \\
\hline PanWU01x14_asm01_ann01_270290 & - & None & FALSE \\
\hline PanWU01x14_asm01_ann01_066330 & - & Glycosyl hydrolase & FALSE \\
\hline PanWU01x14_asm01_ann01_149700 & - & None & FALSE \\
\hline PanWU01x14_asm01_ann01_190510 & - & Small auxin-up RNA & FALSE \\
\hline PanWU01x14_asm01_ann01_124800 & - & The fantastic four family & FALSE \\
\hline PanWU01x14_asm01_ann01_354340 & - & Cytochrome P450, E-class, group I & FALSE \\
\hline PanWU01x14_asm01_ann01_267840 & - & UDP-glucuronosyl/UDP-glucosyltransferase & FALSE \\
\hline PanWU01x14_asm01_ann01_223830 & - & Germin & FALSE \\
\hline PanWU01x14_asm01_ann01_284440 & - & O-methyltransferase & FALSE \\
\hline PanWU01x14_asm01_ann01_177610 & - & CBS domain containing protein & FALSE \\
\hline PanWU01x14_asm01_ann01_004800 & - & FkbH domain containing protein & FALSE \\
\hline PanWU01x14_asm01_ann01_127880 & - & GUN4-like & FALSE \\
\hline PanWU01x14_asm01_ann01_182200 & - & None & FALSE \\
\hline PanWU01x14_asm01_ann01_182190 & - & None & FALSE \\
\hline
\end{tabular}




\begin{tabular}{|c|c|c|c|}
\hline PanWU01x14_asm01_ann01_351880 & - & None & FALSE \\
\hline PanWU01x14_asm01_ann01_340500 & - & Patatin-related protein & FALSE \\
\hline PanWU01x14_asm01_ann01_089360 & - & Self-incompatibility protein & FALSE \\
\hline PanWU01x14_asm01_ann01_100950 & - & late embryogenesis abundant protein & FALSE \\
\hline PanWU01x14_asm01_ann01_174880 & - & Glutathione S-transferase, C-terminal-like & FALSE \\
\hline PanWU01x14_asm01_ann01_216470 & - & Lateral organ boundaries domain containg protein & FALSE \\
\hline PanWU01x14_asm01_ann01_339400 & - & Proton-dependent oligopeptide transporter family & FALSE \\
\hline PanWU01x14_asm01_ann01_099000 & - & Major intrinsic protein & FALSE \\
\hline PanWU01x14_asm01_ann01_233810 & - & Octamer-binding transcription factor & FALSE \\
\hline PanWU01x14_asm01_ann01_014470 & - & DNA polymerase & FALSE \\
\hline PanWU01x14_asm01_ann01_214300 & - & ATP-binding protein & FALSE \\
\hline PanWU01x14_asm01_ann01_345090 & - & Sugar/inositol transporter & FALSE \\
\hline PanWU01x14_asm01_ann01_040390 & - & Acid phosphatase & FALSE \\
\hline PanWU01x14_asm01_ann01_047780 & - & Methylthioribose kinase & FALSE \\
\hline PanWU01x14_asm01_ann01_283100 & - & Small heat shock protein HSP & FALSE \\
\hline PanWU01x14_asm01_ann01_076090 & - & PGG domain containing protein & FALSE \\
\hline PanWU01x14_asm01_ann01_102650 & - & S-crystallin & FALSE \\
\hline PanWU01x14_asm01_ann01_203810 & - & Cellulose synthase & FALSE \\
\hline PanWU01x14_asm01_ann01_367460 & - & Proteinase inhibitor & FALSE \\
\hline PanWU01x14_asm01_ann01_007980 & - & Heavy metal-associated domain containing protein & FALSE \\
\hline PanWU01x14_asm01_ann01_253150 & - & Transferase & FALSE \\
\hline PanWU01x14_asm01_ann01_307320 & - & Plastocyanin & FALSE \\
\hline PanWU01x14_asm01_ann01_062840 & - & Probable membrane-associated kinase regulator & FALSE \\
\hline PanWU01x14_asm01_ann01_216200 & - & Mitogen-activated protein kinase kinase kinase & FALSE \\
\hline PanWU01x14_asm01_ann01_194640 & - & None & FALSE \\
\hline PanWU01x14_asm01_ann01_308800 & - & None & FALSE \\
\hline PanWU01x14_asm01_ann01_296480 & - & EVE domain containing protein & FALSE \\
\hline PanWU01x14_asm01_ann01_010230 & - & UDP-glucuronosyl/UDP-glucosyltransferase & FALSE \\
\hline PanWU01x14_asm01_ann01_231770 & - & Lupus La protein & FALSE \\
\hline PanWU01x14_asm01_ann01_072170 & - & Hopanoid-associated sugar epimerase & FALSE \\
\hline PanWU01x14_asm01_ann01_168000 & - & Glycoside hydrolase & FALSE \\
\hline PanWU01x14_asm01_ann01_080530 & - & Transferase & FALSE \\
\hline PanWU01x14_asm01_ann01_169010 & - & Small GTP-binding domain containing protein & FALSE \\
\hline PanWU01x14_asm01_ann01_161450 & - & L-ascorbate oxidase & FALSE \\
\hline PanWU01x14_asm01_ann01_176520 & - & Zinc finger, LSD1-type & FALSE \\
\hline PanWU01x14_asm01_ann01_322930 & - & SOUL haem-binding protein & FALSE \\
\hline PanWU01x14_asm01_ann01_322710 & - & Glutathione S-transferase, C-terminal-like & FALSE \\
\hline PanWU01x14_asm01_ann01_282030 & - & Protein YLS & FALSE \\
\hline PanWU01x14_asm01_ann01_282460 & - & Parvalbumin & FALSE \\
\hline PanWU01x14_asm01_ann01_067620 & - & None & FALSE \\
\hline PanWU01x14_asm01_ann01_135110 & - & Lipopolysaccharide-modifying protein & FALSE \\
\hline PanWU01x14_asm01_ann01_262250 & - & None & FALSE \\
\hline PanWU01x14_asm01_ann01_009780 & - & Cytochrome P450, E-class, group I & FALSE \\
\hline PanWU01x14_asm01_ann01_214840 & - & DYW domain containing protein & FALSE \\
\hline PanWU01x14_asm01_ann01_061220 & - & Xanthine/uracil/vitamin C permease & FALSE \\
\hline PanWU01x14_asm01_ann01_071810 & - & Peptidase M1, alanine aminopeptidase/leukotriene A4 hydrolase & FALSE \\
\hline PanWU01x14_asm01_ann01_109850 & - & Tyrosine-protein kinase & FALSE \\
\hline PanWU01x14_asm01_ann01_042310 & - & Sugar/inositol transporter & FALSE \\
\hline PanWU01x14_asm01_ann01_371260 & - & Gnk2-like domain containing protein & FALSE \\
\hline PanWU01x14_asm01_ann01_094540 & - & Beta-hexosaminidase & FALSE \\
\hline PanWU01x14_asm01_ann01_258060 & - & S-adenosyl-L-methionine-dependent methyltransferase & FALSE \\
\hline PanWU01x14_asm01_ann01_167320 & - & CASP-like protein $4 \mathrm{~A}$ & FALSE \\
\hline PanWU01x14_asm01_ann01_298980 & - & None & FALSE \\
\hline PanWU01x14_asm01_ann01_119670 & - & Transferase & FALSE \\
\hline PanWU01x14_asm01_ann01_334410 & - & Splicing factor-like protein & FALSE \\
\hline PanWU01x14_asm01_ann01_330480 & - & None & FALSE \\
\hline PanWU01x14_asm01_ann01_044040 & - & P-loop containing nucleoside triphosphate hydrolase & FALSE \\
\hline PanWU01x14_asm01_ann01_299370 & - & Coatomer beta subunit & FALSE \\
\hline PanWU01x14_asm01_ann01_202790 & - & Protein RETICULATA-related & FALSE \\
\hline PanWU01x14_asm01_ann01_074110 & - & Mitochondrial carrier domain containing protein & FALSE \\
\hline
\end{tabular}


Supplemental Table 2. Genes consistently lower expressed in roots of Parasponia compared to Trema.

\begin{tabular}{|c|c|c|c|}
\hline GenelD & Gene name & Functional annotation & $\begin{array}{c}\text { Nodule } \\
\text { enhanced }\end{array}$ \\
\hline PanWU01x14_asm01_ann01_176030 & PanYUCCA4 & Flavin monooxygenase-like enzyme & TRUE \\
\hline PanWU01x14_asm01_ann01_057360 & PanYSL6 & Oligopeptide transporter & TRUE \\
\hline PanWU01x14_asm01_ann01_045060 & PanUGT7 & UDP-glucuronosyl/UDP-glucosyltransferase & TRUE \\
\hline PanWU01x14_asm01_ann01_005340 & PanSURE3 & Survival protein SurE-like phosphatase/nucleotidase & TRUE \\
\hline PanWU01x14_asm01_ann01_243640 & PanDXS3 & Deoxyxylulose-5-phosphate synthase & TRUE \\
\hline PanWU01x14_asm01_ann01_208890 & PanBIN5 & Glycogen synthase kinase & TRUE \\
\hline PanWU01x14_asm01_ann01_183830 & - & NADH:flavin oxidoreductase/NADH oxidase, N-terminal & TRUE \\
\hline PanWU01x14_asm01_ann01_184920 & - & Epoxide hydrolase-like & TRUE \\
\hline PanWU01x14_asm01_ann01_085860 & - & Isopenicillin N synthase & TRUE \\
\hline PanWU01x14_asm01_ann01_105840 & - & Cytochrome P450, E-class, group I & TRUE \\
\hline PanWU01x14_asm01_ann01_323110 & - & Laccase & TRUE \\
\hline PanWU01x14_asm01_ann01_193920 & - & Cellulose synthase & TRUE \\
\hline PanWU01x14_asm01_ann01_215750 & - & Aconitase/Iron-responsive element-binding protein & TRUE \\
\hline PanWU01x14_asm01_ann01_181710 & - & Cytochrome P450, E-class, group I & TRUE \\
\hline PanWU01x14_asm01_ann01_114750 & - & PGAP2-interacting protein & TRUE \\
\hline PanWU01x14_asm01_ann01_171320 & - & ATP-binding cassette containing protein & TRUE \\
\hline PanWU01x14_asm01_ann01_267940 & - & Glycerol-3-phosphate dehydrogenase, NAD-dependent & TRUE \\
\hline PanWU01x14_asm01_ann01_167690 & - & Multi antimicrobial extrusion protein & TRUE \\
\hline PanWU01x14_asm01_ann01_194480 & - & Acid phosphatase & TRUE \\
\hline PanWU01x14_asm01_ann01_177490 & - & CRAL-TRIO lipid binding domain containing protein & TRUE \\
\hline PanWU01x14_asm01_ann01_137540 & - & Cdk-activating kinase assembly factor & TRUE \\
\hline PanWU01x14_asm01_ann01_003680 & - & Xyloglucan endotransglucosylase/hydrolase & TRUE \\
\hline PanWU01x14_asm01_ann01_248020 & - & Helicase, C-terminal & TRUE \\
\hline PanWU01x14_asm01_ann01_359310 & - & Xyloglucan endotransglucosylase/hydrolase & TRUE \\
\hline PanWU01x14_asm01_ann01_275440 & - & protein rolling protein & TRUE \\
\hline PanWU01x14_asm01_ann01_229670 & - & Transglycosylase SLT domain & TRUE \\
\hline PanWU01x14_asm01_ann01_311600 & - & Protein rough sheath 2/ASYMMETRIC LEAVES & TRUE \\
\hline PanWU01x14_asm01_ann01_146560 & - & regulator of nonsense transcript protein & TRUE \\
\hline PanWU01x14_asm01_ann01_084630 & - & TPX & TRUE \\
\hline PanWU01x14_asm01_ann01_268730 & - & Exostosin-like & TRUE \\
\hline PanWU01x14_asm01_ann01_367950 & - & Thioredoxin-like fold containing protein & TRUE \\
\hline PanWU01x14_asm01_ann01_070150 & - & Fructose-1,6-bisphosphatase & TRUE \\
\hline PanWU01x14_asm01_ann01_278960 & - & Solute carrier & TRUE \\
\hline PanWU01x14_asm01_ann01_150310 & - & UDP-glucuronosyl/UDP-glucosyltransferase & TRUE \\
\hline PanWU01x14_asm01_ann01_322230 & - & Tricorn protease & TRUE \\
\hline PanWU01x14_asm01_ann01_036350 & PanWAT38 & Plant-drug/metabolite exporter & FALSE \\
\hline PanWU01x14_asm01_ann01_194430 & PanVSP7 & Acid phosphatase & FALSE \\
\hline PanWU01x14_asm01_ann01_267470 & PanVSP4 & Acid phosphatase & FALSE \\
\hline PanWU01x14_asm01_ann01_078390 & PanPGP5 & $\mathrm{ABC}$ transporter & FALSE \\
\hline PanWU01x14_asm01_ann01_123470 & PanPGP24 & $\mathrm{ABC}$ transporter & FALSE \\
\hline PanWU01x14_asm01_ann01_183880 & PanOPR1 & NADH:flavin oxidoreductase/NADH oxidase & FALSE \\
\hline PanWU01x14_asm01_ann01_260150 & PanOPCL9 & 4-coumarate-CoA ligase & FALSE \\
\hline PanWU01x14_asm01_ann01_335420 & PanNPF41 & Proton-dependent oligopeptide transporter & FALSE \\
\hline PanWU01x14_asm01_ann01_197500 & PanMES5 & Methyl esterase & FALSE \\
\hline PanWU01x14_asm01_ann01_292640 & PanCRK5 & Cysteine rich receptor like kinase & FALSE \\
\hline PanWU01x14_asm01_ann01_313040 & PanBHLH19 & Basic helix-loop-helix transcription factor & FALSE \\
\hline PanWU01x14_asm01_ann01_227120 & - & S-locus glycoprotein & FALSE \\
\hline PanWU01x14_asm01_ann01_079720 & - & NB-ARC domain, LRR domain containing protein & FALSE \\
\hline PanWU01x14_asm01_ann01_141780 & - & Bet v I type allergen & FALSE \\
\hline PanWU01x14_asm01_ann01_118770 & - & Disease resistance response protein & FALSE \\
\hline PanWU01x14_asm01_ann01_197810 & - & B3 DNA binding domain containing protein & FALSE \\
\hline PanWU01x14_asm01_ann01_180630 & - & Small heat shock protein HSP & FALSE \\
\hline PanWU01x14_asm01_ann01_313510 & - & UDP-glucuronosyl/UDP-glucosyltransferase & FALSE \\
\hline PanWU01x14_asm01_ann01_250540 & - & Tyrosine-protein kinase & FALSE \\
\hline PanWU01x14_asm01_ann01_202580 & - & None & FALSE \\
\hline PanWU01x14_asm01_ann01_328800 & - & Small auxin-up RNA & FALSE \\
\hline PanWU01x14_asm01_ann01_183760 & - & UDP-glucuronosyl/UDP-glucosyltransferase & FALSE \\
\hline PanWU01x14_asm01_ann01_104470 & - & Transferase & FALSE \\
\hline
\end{tabular}




\begin{tabular}{|c|c|c|c|}
\hline PanWU01x14_asm01_ann01_077580 & - & Gibberellin regulated protein & FALSE \\
\hline PanWU01x14_asm01_ann01_272960 & - & Phospholipase-like & FALSE \\
\hline PanWU01x14_asm01_ann01_081980 & - & F-box domain containing protein & FALSE \\
\hline PanWU01x14_asm01_ann01_082000 & - & None & FALSE \\
\hline PanWU01x14_asm01_ann01_177330 & - & Isopenicillin N synthase & FALSE \\
\hline PanWU01x14_asm01_ann01_036920 & - & S-receptor-like serine/threonine-protein kinase & FALSE \\
\hline PanWU01x14_asm01_ann01_054610 & - & Cysteine synthase & FALSE \\
\hline PanWU01x14_asm01_ann01_303090 & - & Transferase & FALSE \\
\hline PanWU01x14_asm01_ann01_194000 & - & NB-ARC domain, LRR domain containing protein & FALSE \\
\hline PanWU01x14_asm01_ann01_293230 & - & UDP-glucuronosyl/UDP-glucosyltransferase & FALSE \\
\hline PanWU01x14_asm01_ann01_282100 & - & Alpha/beta hydrolase fold & FALSE \\
\hline PanWU01x14_asm01_ann01_078060 & - & 1,4-alpha-glucan-branching enzyme & FALSE \\
\hline PanWU01x14_asm01_ann01_163500 & - & Transmembrane protein & FALSE \\
\hline PanWU01x14_asm01_ann01_010260 & - & UDP-glucuronosyl/UDP-glucosyltransferase & FALSE \\
\hline PanWU01x14_asm01_ann01_043100 & - & Very-long-chain 3-ketoacyl-CoA synthase & FALSE \\
\hline PanWU01x14_asm01_ann01_034830 & - & Voltage dependent potassium channel & FALSE \\
\hline PanWU01x14_asm01_ann01_029260 & - & Acid phosphatase & FALSE \\
\hline PanWU01x14_asm01_ann01_115130 & - & Calcium-dependent channel & FALSE \\
\hline PanWU01x14_asm01_ann01_099690 & - & Carotenoid oxygenase & FALSE \\
\hline PanWU01x14_asm01_ann01_080220 & - & S-crystallin & FALSE \\
\hline PanWU01x14_asm01_ann01_276120 & - & LRR domain containing protein & FALSE \\
\hline PanWU01x14_asm01_ann01_093390 & - & Multi antimicrobial extrusion protein & FALSE \\
\hline PanWU01x14_asm01_ann01_168510 & - & LRR domain containing protein & FALSE \\
\hline PanWU01x14_asm01_ann01_031700 & - & BURP domain containing protein & FALSE \\
\hline PanWU01x14_asm01_ann01_031750 & - & BURP domain containing protein & FALSE \\
\hline PanWU01x14_asm01_ann01_068150 & - & Avr9/Cf-9 rapidly elicited protein & FALSE \\
\hline PanWU01x14_asm01_ann01_031690 & - & Organ specific protein & FALSE \\
\hline PanWU01x14_asm01_ann01_238590 & - & None & FALSE \\
\hline PanWU01x14_asm01_ann01_252760 & - & Amino acid/polyamine transporter & FALSE \\
\hline PanWU01x14_asm01_ann01_345560 & - & Mono-functional heme-containing catalase & FALSE \\
\hline PanWU01x14_asm01_ann01_216870 & - & GAMYB transcription factor & FALSE \\
\hline PanWU01x14_asm01_ann01_270840 & - & Spastin & FALSE \\
\hline PanWU01x14_asm01_ann01_226680 & - & Protein phosphatase & FALSE \\
\hline PanWU01x14_asm01_ann01_197430 & - & CRIB domain containing protein & FALSE \\
\hline PanWU01x14_asm01_ann01_326690 & - & Alcohol dehydrogenase superfamily, zinc-type & FALSE \\
\hline PanWU01x14_asm01_ann01_087890 & - & Serine/threonine protein kinase & FALSE \\
\hline PanWU01x14_asm01_ann01_168580 & - & Pyruvate kinase & FALSE \\
\hline PanWU01x14_asm01_ann01_312720 & - & Glycoside hydrolase & FALSE \\
\hline PanWU01x14_asm01_ann01_258360 & - & UDP-glucuronosyl/UDP-glucosyltransferase & FALSE \\
\hline PanWU01x14_asm01_ann01_309070 & - & Serine hydroxymethyltransferase & FALSE \\
\hline PanWU01x14_asm01_ann01_227350 & - & Glycoside hydrolase & FALSE \\
\hline PanWU01x14_asm01_ann01_166560 & - & O-methyltransferase COMT-type & FALSE \\
\hline PanWU01x14_asm01_ann01_018930 & - & Universal stress protein & FALSE \\
\hline PanWU01x14_asm01_ann01_001620 & - & Mpv17/PMP & FALSE \\
\hline PanWU01x14_asm01_ann01_128500 & - & Peroxidase & FALSE \\
\hline PanWU01x14_asm01_ann01_142560 & - & Chloride channel, voltage gated & FALSE \\
\hline PanWU01x14_asm01_ann01_182300 & - & S-adenosyl-L-methionine-dependent methyltransferase & FALSE \\
\hline PanWU01x14_asm01_ann01_271740 & - & Amidohydrolase & FALSE \\
\hline PanWU01x14_asm01_ann01_067000 & - & Cellulose synthase & FALSE \\
\hline PanWU01x14_asm01_ann01_085420 & - & Six-hairpin glycosidase & FALSE \\
\hline PanWU01x14_asm01_ann01_133570 & - & Amino acid transporter, transmembrane domain containing protein & FALSE \\
\hline PanWU01x14_asm01_ann01_256680 & - & Parvalbumin & FALSE \\
\hline PanWU01x14_asm01_ann01_219030 & - & Sugar/inositol transporter & FALSE \\
\hline PanWU01x14_asm01_ann01_006390 & - & Alpha/Beta hydrolase fold containing protein & FALSE \\
\hline PanWU01x14_asm01_ann01_235980 & - & Major latex protein domain containing protein & FALSE \\
\hline PanWU01x14_asm01_ann01_235990 & - & Bet $v$ I type allergen & FALSE \\
\hline PanWU01x14_asm01_ann01_107640 & - & Voltage dependent potassium channel & FALSE \\
\hline PanWU01x14_asm01_ann01_305640 & - & Mitochondrial pyruvate carrier & FALSE \\
\hline PanWU01x14_asm01_ann01_148530 & - & D-ribose-binding periplasmic protein & FALSE \\
\hline PanWU01x14_asm01_ann01_306440 & - & Aspartate/other aminotransferase & FALSE \\
\hline PanWU01x14_asm01_ann01_249190 & - & Diacylglycerol O-acyltransferase & FALSE \\
\hline PanWU01x14_asm01_ann01_228960 & - & Lipase & FALSE \\
\hline
\end{tabular}




\begin{tabular}{|c|c|c|c|}
\hline PanWU01x14_asm01_ann01_045710 & - & $\mathrm{N}$-acyl-L-amino-acid amidohydrolase & FALSE \\
\hline PanWU01x14_asm01_ann01_177520 & - & None & FALSE \\
\hline PanWU01x14_asm01_ann01_034460 & - & Glycosyl transferase & FALSE \\
\hline PanWU01x14_asm01_ann01_014760 & - & Serine/threonine protein kinase & FALSE \\
\hline PanWU01x14_asm01_ann01_281660 & - & Carbonic anhydrase, alpha-class & FALSE \\
\hline PanWU01x14_asm01_ann01_317730 & - & Ternary complex factor MIP1, leucine-zipper & FALSE \\
\hline PanWU01x14_asm01_ann01_167890 & - & Splicing factor-like protein & FALSE \\
\hline PanWU01x14_asm01_ann01_108930 & - & 43kDa postsynaptic protein & FALSE \\
\hline PanWU01x14_asm01_ann01_084470 & - & Glycoside hydrolase & FALSE \\
\hline PanWU01x14_asm01_ann01_084440 & - & Very-long-chain 3-ketoacyl-CoA synthase & FALSE \\
\hline PanWU01x14_asm01_ann01_099650 & - & Guanine nucleotide-binding protein, beta subunit & FALSE \\
\hline PanWU01x14_asm01_ann01_133790 & - & Pectin lyase fold/virulence factor & FALSE \\
\hline PanWU01x14_asm01_ann01_331600 & - & ER lumen protein retaining receptor & FALSE \\
\hline PanWU01x14_asm01_ann01_112690 & - & Peroxiredoxin Q & FALSE \\
\hline PanWU01x14_asm01_ann01_038900 & - & Sm-like protein Lsm6/SmF & FALSE \\
\hline PanWU01x14_asm01_ann01_133560 & - & Amino acid transporter, transmembrane domain containing protein & FALSE \\
\hline PanWU01x14_asm01_ann01_112410 & - & None & FALSE \\
\hline PanWU01x14_asm01_ann01_012030 & - & Universal stress protein & FALSE \\
\hline PanWU01x14_asm01_ann01_079000 & - & Glycogen/starch/alpha-glucan phosphorylase & FALSE \\
\hline PanWU01x14_asm01_ann01_048650 & - & Glycoside hydrolase & FALSE \\
\hline PanWU01x14_asm01_ann01_104000 & - & Magnesium-protoporphyrin IX methyltransferase & FALSE \\
\hline PanWU01x14_asm01_ann01_222720 & - & Short-chain dehydrogenase/reductase & FALSE \\
\hline PanWU01x14_asm01_ann01_274170 & - & Ubiquitin-activating enzyme $\mathrm{E}$ & FALSE \\
\hline PanWU01x14_asm01_ann01_200550 & - & Pentatricopeptide repeat & FALSE \\
\hline PanWU01x14_asm01_ann01_038010 & - & Chlorophyllase & FALSE \\
\hline PanWU01x14_asm01_ann01_251780 & - & $18 \mathrm{~S}$ pre-ribosomal assembly protein gar2-like protein & FALSE \\
\hline PanWU01x14_asm01_ann01_188030 & - & Protein prenyltransferase, alpha subunit & FALSE \\
\hline PanWU01x14_asm01_ann01_156170 & - & PsbP family & FALSE \\
\hline PanWU01x14_asm01_ann01_121420 & - & None & FALSE \\
\hline PanWU01x14_asm01_ann01_274960 & - & Tyrosine-protein kinase & FALSE \\
\hline PanWU01x14_asm01_ann01_352370 & - & Serine/threonine protein kinase & FALSE \\
\hline PanWU01x14_asm01_ann01_055290 & - & 3-dehydroquinate dehydratase type I & FALSE \\
\hline PanWU01x14_asm01_ann01_261960 & - & Protein phosphatase & FALSE \\
\hline PanWU01x14_asm01_ann01_286000 & - & None & FALSE \\
\hline PanWU01x14_asm01_ann01_329750 & - & Tetratricopeptide-like helical domain containing protein & FALSE \\
\hline PanWU01x14_asm01_ann01_078300 & - & DedA family & FALSE \\
\hline PanWU01x14_asm01_ann01_343020 & - & WEB family & FALSE \\
\hline PanWU01x14_asm01_ann01_274780 & - & Armadillo-type fold containing protein & FALSE \\
\hline PanWU01x14_asm01_ann01_194960 & - & EMSY N-terminal & FALSE \\
\hline PanWU01x14_asm01_ann01_157910 & - & None & FALSE \\
\hline PanWU01x14_asm01_ann01_312640 & - & None & FALSE \\
\hline PanWU01x14_asm01_ann01_225140 & - & ATP-binding cassette containing protein & FALSE \\
\hline PanWU01x14_asm01_ann01_332850 & - & None & FALSE \\
\hline PanWU01x14_asm01_ann01_249880 & - & Non-haem dioxygenase $\mathrm{N}$-terminal domain containing protein & FALSE \\
\hline PanWU01x14_asm01_ann01_021790 & - & AWPM-19-like & FALSE \\
\hline PanWU01x14_asm01_ann01_190080 & - & Amidase & FALSE \\
\hline PanWU01x14_asm01_ann01_108820 & - & Tricorn protease & FALSE \\
\hline PanWU01x14_asm01_ann01_356640 & - & F-box domain containing protein & FALSE \\
\hline PanWU01x14_asm01_ann01_371870 & - & F-box domain containing protein & FALSE \\
\hline PanWU01x14_asm01_ann01_086200 & - & Glycolipid transfer protein domain containing protein & FALSE \\
\hline PanWU01x14_asm01_ann01_153820 & - & Voltage dependent potassium channel & FALSE \\
\hline PanWU01x14_asm01_ann01_235350 & - & Serine/threonine protein kinase & FALSE \\
\hline PanWU01x14_asm01_ann01_227480 & - & Lung seven transmembrane receptor-like & FALSE \\
\hline PanWU01x14_asm01_ann01_299310 & - & $\begin{array}{l}\text { Peptidase S9, prolyl oligopeptidase, catalytic domain containing } \\
\text { protein }\end{array}$ & FALSE \\
\hline PanWU01x14_asm01_ann01_227340 & - & Glycoside hydrolase & FALSE \\
\hline PanWU01x14_asm01_ann01_200710 & - & Cytochrome P450, E-class, group II & FALSE \\
\hline PanWU01x14_asm01_ann01_331960 & - & F-box associated domain, type & FALSE \\
\hline PanWU01x14_asm01_ann01_269470 & - & Glyceraldehyde/Erythrose phosphate dehydrogenase family & FALSE \\
\hline PanWU01x14_asm01_ann01_336400 & - & Stomatin family & FALSE \\
\hline PanWU01x14_asm01_ann01_095570 & - & LRR domain containing protein & FALSE \\
\hline PanWU01x14_asm01_ann01_203500 & - & Sieve element occlusion, C-terminal & FALSE \\
\hline
\end{tabular}




\begin{tabular}{|c|c|c|c|}
\hline PanWU01x14_asm01_ann01_203490 & - & Sieve element occlusion & FALSE \\
\hline PanWU01x14_asm01_ann01_198660 & - & WD40/YVTN repeat-like-containing domain containing protein & FALSE \\
\hline PanWU01x14_asm01_ann01_169890 & - & SGNH hydrolase-type esterase domain containing protein & FALSE \\
\hline PanWU01x14_asm01_ann01_357800 & - & LRR domain containing protein & FALSE \\
\hline PanWU01x14_asm01_ann01_142010 & - & None & FALSE \\
\hline PanWU01x14_asm01_ann01_332760 & - & Pectin lyase fold containing protein & FALSE \\
\hline PanWU01x14_asm01_ann01_215770 & - & Aspartate decarboxylase & FALSE \\
\hline PanWU01x14_asm01_ann01_312620 & - & None & FALSE \\
\hline PanWU01x14_asm01_ann01_177510 & - & None & FALSE \\
\hline PanWU01x14_asm01_ann01_243880 & - & WAT1-related protein & FALSE \\
\hline PanWU01x14_asm01_ann01_230560 & - & Sugar/inositol transporter & FALSE \\
\hline PanWU01x14_asm01_ann01_230940 & - & None & FALSE \\
\hline PanWU01x14_asm01_ann01_292790 & - & None & FALSE \\
\hline PanWU01x14_asm01_ann01_263290 & - & None & FALSE \\
\hline PanWU01x14_asm01_ann01_310030 & - & 14-3-3 protein & FALSE \\
\hline PanWU01x14_asm01_ann01_310320 & - & UDP-glucuronosyl/UDP-glucosyltransferase & FALSE \\
\hline PanWU01x14_asm01_ann01_108830 & - & None & FALSE \\
\hline PanWU01x14_asm01_ann01_314470 & - & Caleosin-related & FALSE \\
\hline PanWU01x14_asm01_ann01_319930 & - & Small GTPase superfamily, Ras type & FALSE \\
\hline PanWU01x14_asm01_ann01_163320 & - & Cytochrome P & FALSE \\
\hline PanWU01x14_asm01_ann01_158460 & - & None & FALSE \\
\hline PanWU01x14_asm01_ann01_157170 & - & None & FALSE \\
\hline PanWU01x14_asm01_ann01_157940 & - & None & FALSE \\
\hline PanWU01x14_asm01_ann01_157930 & - & Major facilitator & FALSE \\
\hline PanWU01x14_asm01_ann01_220370 & - & None & FALSE \\
\hline PanWU01x14_asm01_ann01_241640 & - & Serine/threonine protein kinase & FALSE \\
\hline PanWU01x14_asm01_ann01_067750 & - & Transferase & FALSE \\
\hline PanWU01x14_asm01_ann01_072200 & - & None & FALSE \\
\hline PanWU01x14_asm01_ann01_070210 & - & None & FALSE \\
\hline PanWU01x14_asm01_ann01_271750 & - & Amidohydrolase & FALSE \\
\hline
\end{tabular}

Supplemental Table 3. Multiple reactions monitoring (MRM) transitions table for all hormones and corresponding internal standards used in this study.

\begin{tabular}{|c|c|c|c|c|c|}
\hline Compound & $\begin{array}{l}\text { Retention time } \\
\text { (min) }\end{array}$ & Scan mode & $\begin{array}{c}\text { MRM transition } \\
\mathrm{m} / \mathrm{z}\end{array}$ & Cone voltage (V) & $\begin{array}{l}\text { Collision } \\
\text { energy }(e V)\end{array}$ \\
\hline$J A$ & 8.63 & Negative & $208.94>58.95$ & 22 & 15 \\
\hline$\left[{ }^{2} \mathrm{H}_{6}\right]-\mathrm{JA}$ & 8.52 & Negative & $214.74>58.82$ & 22 & 20 \\
\hline SA & 4.54 & Negative & $136.8>92.95$ & 36 & 15 \\
\hline$\left[{ }^{2} \mathrm{H}_{4}\right]-\mathrm{SA}$ & 4.44 & Negative & $141.1>96.8$ & 36 & 15 \\
\hline JA-Ile & 11.87 & Positive & $324.33>278.24$ & 36 & 15 \\
\hline$\left[{ }^{2} \mathrm{H}_{2}\right]-J A-\| l e$ & 11.84 & Positive & $326.16>280.06$ & 15 & 15 \\
\hline OPDA & 16.31 & Positive & $293.3>275.3$ & 36 & 10 \\
\hline$\left[{ }^{2} \mathrm{H}_{5}\right]-\mathrm{OPDA}$ & 16.26 & Positive & $298.22>279.3$ & 36 & 10 \\
\hline ABA & 6.86 & Negative & $263.06>153.07$ & 36 & 15 \\
\hline$\left[{ }^{2} \mathrm{H}_{6}\right]-\mathrm{ABA}$ & 6.78 & Negative & $269.2>159.1$ & 27 & 15 \\
\hline IAA & 4.72 & Positive & $176.28>130.2$ & 32 & 15 \\
\hline$\left[{ }^{2} \mathrm{H}_{5}\right]-I A A$ & 4.72 & Positive & $182.1>109.08$ & 34 & 25 \\
\hline
\end{tabular}


Supplemental Table 4. Primers used in this study.

\begin{tabular}{|c|c|c|}
\hline Name & Purpose & Sequence \\
\hline PanLOX2_sgRNA1 & Clone sgRNA & $\begin{array}{c}\text { tgtggtctcaattGACAAACCTTAATTTACAGCG } \\
\text { gttttagagctagaaatagcaag }\end{array}$ \\
\hline PanLOX2_sgRNA2 & Clone sgRNA & $\begin{array}{c}\text { tgtggtctcaattGTTGTAAGGGAACATAATAGA } \\
\text { gttttagagctagaaatagcaag }\end{array}$ \\
\hline PanLOX2_sgRNA3 & Clone sgRNA & $\begin{array}{c}\text { tgtggtctcaattGTATAACCGTCAAGCGTACTG } \\
\text { gttttagagctagaaatagcaag }\end{array}$ \\
\hline PanLOX3_sgRNA1 & Clone sgRNA & $\begin{array}{c}\text { tgtggtctcaattGAAACGATGTCGTTTCGGCA } \\
\text { gttttagagctagaaatagcaag }\end{array}$ \\
\hline PanLOX3_sgRNA2 & Clone sgRNA & $\begin{array}{c}\text { tgtggtctcaattGTGTCTTACCGACCCTCGGAG } \\
\text { gttttagagctagaaatagcaag }\end{array}$ \\
\hline PanLOX3_sgRNA3 & Clone sgRNA & $\begin{array}{c}\text { tgtggtctcaattGTCGCCTCTCCAACATCCAGC } \\
\text { gttttagagctagaaatagcaag }\end{array}$ \\
\hline PanAOC1_sgRNA1 & Clone sgRNA & $\begin{array}{c}\text { tgtggtctcaattGAAGAAGATAACGGTGATCTG } \\
\text { gttttagagctagaaatagcaag }\end{array}$ \\
\hline PanAOC1_sgRNA2 & Clone sgRNA & $\begin{array}{c}\text { tgtggtctcaattGGTTTGGGATCTTACTAAAG } \\
\text { gttttagagctagaaatagcaag }\end{array}$ \\
\hline PanAOC1_sgRNA3 & Clone sgRNA & $\begin{array}{c}\text { tgtggtctcaattGGAGACTTGCAAAAAAGGAT } \\
\text { gttttagagctagaaatagcaag }\end{array}$ \\
\hline PanAOC2_sgRNA1 & Clone sgRNA & $\begin{array}{c}\text { tgtggtctcaattGAGCGATGTCGTTTTGAGAG } \\
\text { gttttagagctagaaatagcaag }\end{array}$ \\
\hline PanAOC2_sgRNA2 & Clone sgRNA & $\begin{array}{c}\text { tgtggtctcaattGTATTGATGAGAGTTTAAGGG } \\
\text { gttttagagctagaaatagcaag }\end{array}$ \\
\hline PanAOC2_sgRNA3 & Clone sgRNA & $\begin{array}{c}\text { tgtggtctcaattGCGAGACAGGGGAAGTCCGG } \\
\text { gttttagagctagaaatagcaag }\end{array}$ \\
\hline PanJAR1_sgRNA1 & Clone sgRNA & $\begin{array}{c}\text { tgtggtctcaattGAGAAGTCCTATATATCTGCA } \\
\text { gttttagagctagaaatagcaag }\end{array}$ \\
\hline PanJAR1_sgRNA2 & Clone sgRNA & $\begin{array}{c}\text { tgtggtctcaattGGAATTTGAAGCTTTAACAG } \\
\text { gttttagagctagaaatagcaag }\end{array}$ \\
\hline PanJAR1_sgRNA3 & Clone sgRNA & $\begin{array}{c}\text { tgtggtctcaattGAAAAGATGGAAGAGTTCAA } \\
\text { gttttagagctagaaatagcaag }\end{array}$ \\
\hline sgRNA_Rv & Clone sgRNA & tgtggtctcaAGCGTAATGCCAACTTTGTAC \\
\hline geno_PanLOX2-crispr-Fw & Genotyping & GGGATTGAGCTGGCTCTGAA \\
\hline geno_PanLOX2-crispr-Rv & Genotyping & ACTTACCAGGGACGAGGTCA \\
\hline geno_PanLOX3-crispr-Fw & Genotyping & TGTCGCTGTCTACGCATATG \\
\hline geno_PanLOX3-crispr-Rv & Genotyping & TTGGGAATTGTCGGACTTGG \\
\hline geno_PanAOC1-crispr-Fw & Genotyping & ACCACGTGATGAAGTCCACC \\
\hline geno_PanAOC1-crispr-Rv & Genotyping & GATCCCTGGACCGCTATGTG \\
\hline geno_PanAOC2-crispr-Fw & Genotyping & ACACCAGCAAAGGGGGAAAA \\
\hline geno_PanAOC2-crispr-Rv & Genotyping & GGTCGCCGGAGTAGATCTTG \\
\hline geno_PanJAR1-crispr-Fw & Genotyping & TTGGCGTCGTAGTTTTTGGC \\
\hline geno_PanJAR1-crispr-Rv & Genotyping & TCCGCAGAGAAAATGGCAGT \\
\hline
\end{tabular}


Supplemental Table 5. GenelDs for all genes mentioned in this chapter. GenelDs refer to $P$. andersonii gene models, which can be searched for on www.parasponia.org.

\begin{tabular}{|c|c|}
\hline Gene name & GenelD \\
\hline PanACX1 & PanWU01x14_asm01_ann01_276320 \\
\hline PanACX2 & PanWU01x14_asm01_ann01_191150 \\
\hline PanACX4 & PanWU01x14_asm01_ann01_218440 \\
\hline PanAMT4 & PanWU01x14_asm01_ann01_281510 \\
\hline PanAMT5 & PanWU01x14_asm01_ann01_211750 \\
\hline PanAOC1 & PanWU01x14_asm01_ann01_021690 \\
\hline PanAOC2 & PanWU01x14_asm01_ann01_158610 \\
\hline PanAOS1 & PanWU01x14_asm01_ann01_083380 \\
\hline PanAOS3 & PanWU01x14_asm01_ann01_081200 \\
\hline PanAOS4 & PanWU01x14_asm01_ann01_032510 \\
\hline PanHCE7/JAR1 & PanWU01x14_asm01_ann01_283440 \\
\hline PanKAT1 & PanWU01x14_asm01_ann01_129370 \\
\hline PanKAT2 & PanWU01x14_asm01_ann01_216730 \\
\hline PanLOX2 & PanWU01x14_asm01_ann01_085160 \\
\hline PanLOX3 & PanWU01x14_asm01_ann01_085170 \\
\hline PanLOX4 & PanWU01x14_asm01_ann01_345980 \\
\hline PanLOX6 & PanWU01x14_asm01_ann01_208600 \\
\hline PanMFP2 & PanWU01x14_asm01_ann01_142250 \\
\hline PanOPCL1 & PanWU01x14_asm01_ann01_151930 \\
\hline PanOPR3 & PanWU01x14_asm01_ann01_200770 \\
\hline PanPT4 & PanWU01x14_asm01_ann01_225280 \\
\hline PanRAD1 & PanWU01x14_asm01_ann01_257240 \\
\hline PanSTR2 & PanWU01x14_asm01_ann01_309120 \\
\hline PanVPY & PanWU01x14_asm01_ann01_341680 \\
\hline
\end{tabular}





\section{Chapter 7}

General Discussion:

Recruitment of hormonal networks in

nitrogen-fixing nodule symbioses 


\section{Abstract}

Plants require vast amounts of nitrogen for growth and development. To ensure ample nitrogen supply, approximately ten distantly related plant lineages engage endosymbiotically with nitrogen-fixing bacterial species. These associations occur in specialized organs formed on the host root, named nodules. Research on the legume-rhizobium symbiosis showed that perception of rhizobial signals affects plant hormone homeostasis and that multiple hormones are involved in nodule organogenesis. Here, I question whether different nodulating lineages recruited the same hormonal networks to function in nodule formation. Furthermore, I examine whether nodulating species harbor genetic adaptations in hormonal pathways that correlate with nodulation capacity. 


\section{INTRODUCTION}

In natural ecosystems, nutrient availability is a major determinant of plant growth potential. To increase nutrient uptake, plant roots can associate with a diverse array of soil microbes [1]. A well-known example of this is the nodular endosymbiosis between nitrogen-fixing bacterial species and legume plants [2]. This symbiosis is not unique to legumes but is found among ten separate lineages within the orders Fabales, Fagales, Cucurbitales and Rosales that together form the so-called 'nitrogen-fixation clade' [3-6]. Within this clade, legumes (Fabaceae, order Fabales) and Parasponia (Cannabaceae, order Rosales) associate with gram negative $\alpha$-, $\beta$ - and $\gamma$-proteobacteria, collectively named rhizobia [7]. On the other hand, actinorhizal plants, which represent a paraphyletic group of $\sim 220$ species belonging to 25 genera in the Fagales, Cucurbitales and Rosales, interact with gram positive actinomycetes of the genus Frankia [4,8,9].

Despite differences in microbial partner, the nitrogen-fixing symbioses of legumes, Parasponia and actinorhizas are founded on conserved genetic networks. These networks have been largely co-opted from the symbiosis with arbuscular mycorrhizal fungi $[10,11]$. These obligate biotrophic fungi colonize roots of most land plants and form dense hyphal networks inside existing root cortical cells, known as arbuscules [12,13]. Additionally, all nitrogen-fixing symbioses have in common that the microbial symbionts are accommodated intracellularly inside specialized organs that are formed on the host root $[14,15]$. These socalled 'nodules' results from cell divisions that are induced upon perception of microbial signals. In plants, development is regulated by interacting hormonal networks $[16,17]$. This implies that microbe-induced signaling affects hormone homeostasis of the host root, subsequently leading to nodule organogenesis. Indeed, several studies have shown that in legumes perception of rhizobial lipo-chitooligosaccharide (LCO) signals transcriptionally activates biosynthesis and signaling pathways of multiple plant hormones, among which are cytokinins, auxins, strigolactones and gibberellins [18-20] [Chapter 3-4]. Additionally, molecular genetic studies have uncovered essential roles of cytokinin and ethylene in nodule organogenesis and microbial infection [21,22]. Most of these studies have been performed on a handful of model legumes. However, data on species from distantly-related branches of the legume family as well as on Parasponia and actinorhizal species are emerging.

Here, I will discuss the role of hormonal networks in nitrogen-fixing nodule symbioses and focus on two main aspects, namely: I. whether different nodulating lineages convergently recruited the same hormonal networks, and II. whether nodulating species harbor genetic adaptations in hormonal pathways that correlate with nodulation capacity. 


\section{Parallel Use of Hormones in Symbiosis}

\section{Auxin}

A prerequisite for plant organ formation is the formation of a local auxin maximum [23]. These maxima are formed through asymmetric distribution of auxin efflux carriers of the PIN protein family $[23,24]$. Not surprisingly, analysis of auxin-responsive reporters in the model legumes Medicago truncatula (medicago), Lotus japonicus (lotus) and Glycine max (soybean) as well as the actinorhizal species Discaria trinervis (Rhamnaceae, order Rosales) showed that nodule primordia are associated with local auxin response maxima [25-29]. These maxima are often broader and appear more diffuse than those observed during lateral root formation $[25,29,30]$. The mechanistic basis of the formation of such maxima in response to microbial recognition is not well understood. In most legumes as well as some actinorhizal species, nodule organogenesis is induced in the root cortex and/or pericycle in response to epidermal recognition of microbial signals $[9,14,15,31]$. Computer simulations predict that the auxin maxima associated with nodule initiation can be generated through local reduction of auxin transport capacity in the root cortex [32]. These simulations also indicate that a diffusible signal originating from a single epidermal cell is sufficient to cause auxin accumulation through reduction of cortical auxin transport [33]. Experimental observations support these predictions. In medicago, auxin transport capacity is reduced below the nodule initiation site at 24 hours after inoculation [27,34]. Additionally, application of auxin transport inhibitors triggers the formation of nodule-like structures on roots of several legume species [35-39]. Similar structures are observed on medicago roots after treatment with flavonoids [40], which function as natural auxin transport inhibitors [41]. Silencing of a flavonoid biosynthesis gene in the actinorhizal tree Casuarina glauca (Casuarinaceae, order Fagales) reduced nodule number [42]. However, since flavonoids also function as chemo-attractants for Frankia bacteria [43], it cannot be concluded that this results from a direct effect on auxin transport.

Besides auxin export, also activity of auxin import carriers of the AUX/LAX family regulates auxin distribution [44]. The inhibitor 1-naphthoxyacetic acid (1-NOA) interferes with AUX/ LAX auxin import activity [45]. Application of 1-NOA to roots of medicago as well as the actinorhizas $D$. trinervis and C. glauca negatively affects nodule formation $[29,46,47]$. Consistently, Mtlax2 mutants in medicago develop less nodules than the corresponding wild type [46]. Analysis of MtLAX2 promoter activity showed that this gene is expressed in incipient nodule primordia and at later stages in the nodule meristem but is absent from infected root hairs [46]. A similar expression pattern was observed for DtAUX1, a close homolog in D. trinervis [29]. In contrast, in C. glauca CgAUX1 promoter activity is observed 
in infected root hairs and cortical cells but is absent from uninfected primordial cells [47]. At later stages, promoter activity is observed in fully-infected cells of the mature nodule [47]. Surprisingly, expression of the CgAUX1 promoter reporter construct in $D$. trinervis restricts promoter activity to the nodule meristem, as is observed for the promoter of DtAUX1 [29]. This suggests potential differences in regulation and functioning of AUX/LAX-mediated auxin import between different nodulating lineages.

In addition to an important role in nodule organogenesis, recent studies suggest that auxin is also involved in intracellular infection by rhizobium and Frankia bacteria. Transcriptomic studies on epidermal cells treated with rhizobial LCOs or infected by rhizobium bacteria indicated transcriptional activation of auxin biosynthesis and signaling genes $[19,48]$. Additionally, rhizobial inoculation elevated expression of auxin-responsive promoter reporter constructs in medicago root hairs $[40,48]$. The auxin response factor MtARF16a is induced at rhizobial infection sites. Mutation of this gene reduced the number of infection threads, strongly suggesting that auxin signaling is required for rhizobial infection in medicago $[48,49]$. As described above, the expression pattern of $C g A U X 1$ also suggests a role for auxin in Frankia infection of $C$. glauca root hairs and nodules [47]. Immunolocalization detected accumulation of the auxin phenyl-acetic acid (PAA) in infected cells of mature nodules in C. glauca and D. trinervis $[29,47]$. However, the source and subcellular localization of PAA inside these cells is not entirely clear. A source of PAA could be phenyl-acetic hopanetetrol, a specific lipid of Frankia vesicles [50,51]. Therefore, it cannot be excluded that PAA is present in the apoplastic space surrounding the Frankia hyphae, rather than the host cell cytoplasm. The latter is supported by no detectable signal from the auxin-responsive $D R 5$ reporter in $D$. trinervis infected cells [29]. However, during mycorrhizal symbiosis, DR5 signal is detected in arbusculated cells of medicago, tomato (Solanum lycopersicum) and rice (Oryza sativa) [52]. Overexpression of microRNA393 that results in downregulation of auxin receptor genes blocked arbuscule formation [52]. This indicates that auxin perception is required for intracellular infection by mycorrhizal fungi. Additional experimentation is required to determine, whether intracellular colonization by rhizobia or Frankia is equally dependent on auxin signaling.

\section{Cytokinin}

In legumes, cytokinin signaling forms an integral part of the nodulation signaling network. Rhizobium inoculation or treatment with rhizobium LCOs rapidly activates expression of typical cytokinin-responsive genes, such as type-A Response Regulators [19,53-57]. Additionally, LCO treatment or rhizobial inoculation induces accumulation of cytokinin in medicago or lotus roots, respectively $[20,58]$ [Chapter 3$]$. This represents a crucial step in 
LCO signaling, as most of the transcriptional changes induced by rhizobium LCOs at three hours post treatment are dependent on cytokinin perception [20] [Chapter 3]. Mutants in the orthologous cytokinin receptors MtCRE1 in medicago and LjLHK1 in lotus are severely affected in nodule organogenesis $[55,59]$. These mutants only occasionally form nodules, due to redundant function of additional cytokinin receptors $[60,61]$.

Laser dissection-RNAseq revealed transcriptional activation of cytokinin biosynthesis genes as well as genes encoding putative cytokinin transport proteins in medicago root epidermal cells following rhizobium LCO treatment [19]. This suggests that cytokinins might be released form epidermal cells in response to rhizobial recognition to induce nodule initiation in the root cortex. However, cytokinin function during nodule organogenesis appears not to be restricted to legume species that depend on root hair-based infection. Rhizobia infect roots of the tropical legume Aeschynomene evenia intercellularly through cracks in the epidermis and induce cortical cell divisions upon direct contact [62]. Silencing of AeHK1, the A. evenia ortholog of LjLHK1/MtCRE1, substantially reduced the proportion of nodulated roots [63]. This suggests that activation of cytokinin signaling represents a universal step during legume root nodule organogenesis.

In contrast to legumes, cytokinin involvement in root nodule formation in actinorhizas and Parasponia is not well resolved, yet. Mutagenesis of the Parasponia andersonii orthologue of LjLHK1/MtCRE1, named PanHK4, did not affect root nodule formation [Chapter 5]. However, it cannot be excluded that this results from redundant functioning of PanHK2 and PanHK3, two additional cytokinin receptors encoded in the $P$. andersonii genome [64] [Chapter 5]. Preliminary results suggest that absence of a nodulation phenotype for Panhk4 mutants could indeed result from redundant functioning of PanHK2 and PanHK3 during Parasponia nodule formation. On roots of $P$. andersonii RNAi lines in which PanHK2, PanHK3 and PanHK4 are co-silenced, reduced nodule formation is observed (Wardhani et al., personal communication). Therefore, the contribution of cytokinin to Parasponia nodule formation remains unresolved.

Exogenous cytokinin treatment can induce nodule-like structures on the roots of several legume species [65-68]. However, substantial genetic variation exists in this response, even between different ecotypes of lotus [67]. Cytokinin application has been reported to also induce nodule-like structures on roots of the actinorhizal tree alder (Alnus glutinosa, Betulaceae, order Fagales) [69]. This suggests that cytokinin might also be involved in the formation of Frankia nodules on alder roots. However, it should be noted that the structure of these pseudonodules differs from that of genuine alder nodules [69]. The same applies to 
legume nodule-like structures induced by cytokinin application, questioning the relevance of these results. Therefore, it remains to be determined whether cytokinin is equally important to actinorhizal and Parasponia nodule organogenesis as it is to legume nodule development.

\section{Ethylene}

Ethylene is generally considered to be a negative regulator of legume nodule formation and rhizobial infection [70]. This was first shown by pharmacological studies, which showed that application of ethylene or its precursor 1-amino-cyclopropane-1-carboxylic acid (ACC) inhibits nodule formation on roots of several legume species [71-73]. Conversely, application of the ACC synthase inhibitor aminoethoxyvinylglycine (AVG) promotes nodule formation and rhizobial infection [71-77]. Strong support for a negative role of ethylene during the legume-rhizobium symbiosis came from analysis of the medicago Mtein2/Mtskl mutant. This mutant is ethylene insensitive due to a mutation in EIN2, encoding a central component of the ethylene signaling pathway $[71,78,79]$. The Mtein2/Mtskl mutant is hyper-infected, forming a high number of infection threads. Additionally, this mutant forms clusters of small nodules in distinct sickle-shaped zones along the root [31,71]. A similar phenotype is observed in lotus after RNAi-mediated knockdown of both EIN2-encoding genes or overexpressing of mutant ethylene receptors, causing ethylene insensitivity [8082]. The hyper-infection phenotype might result from the absence of negative feedback on LCO-induced signaling. In medicago, several ethylene biosynthesis genes are induced following rhizobial inoculation or LCO treatment [18,20] [Chapter 3]. Likewise, an increase in ethylene release has been measured on alfalfa, Vicia sativa and soybean roots after inoculation with rhizobium [83-85]. Ethylene has been shown to affect symbiotic signaling already at an early point in the LCO signaling cascade [73] and therefore LCO-induced ethylene production might directly inhibit subsequent rhizobial infection. In medicago, ethylene treatment increased the concentration of rhizobium LCOs required to initiate calcium spiking, a response observed within minutes of LCO perception [73]. Additionally, a change in spiking frequency was observed in the ethylene-insensitive Mtein2/Mtsk/ mutant compared to wild type [73]. Consistent with a role for ethylene in regulation of early LCO signaling, transcriptome sequencing revealed a massive de-repression of early symbiotic signaling in inoculated Mtein2/Mtskl roots [18].

Despite an overall negative effect of ethylene on legume nodule formation, differences in the response to ethylene are observed within the legume family. In soybean, genetic variation in response to ethylene treatment exists between cultivars. Whereas some cultivars are highly sensitive and respond with almost complete inhibition of nodule formation, others are unresponsive to ethylene treatment with regard to nodule development $[72,76,86]$. 
In the semiaquatic legume Sesbania rostrata, the effect of ethylene on nodule formation depends on the mode of rhizobial infection [87]. Under aerated conditions, S. rostrata is infected through a root hair-based infection mechanism. Under these conditions, rhizobial infection is negatively controlled by ethylene [88]. In contrast, under flooded conditions rhizobia infect S. rostrata intercellularly in an ethylene-dependent manner [89]. Under these conditions, rhizobia enter the root through epidermal cracks at the site of emerging lateral roots $[90,91]$. Once inside, the rhizobia proliferate in infection pockets that result from LCO-dependent localized cell death. The formation of these infection pockets involves the production of hydrogen peroxide, ethylene and gibberellic acid $[89,92,93]$. The switch between both infection modes is likely also regulated by ethylene, which accumulates under anaerobic conditions [87]. Epidermal cells at the lateral root base generally respond to LCO application with a calcium spiking response that is faster and more symmetrical than observed in susceptible root hairs. Addition of AVG induced asymmetric calcium spikes with a reduced frequency [94]. Additionally, this allowed intracellular infection, even under flooded conditions [88,94]. Ethylene-dependent intercellular infection is also observed in the lotus nena mutant, which is compromised in root hair-based intracellular infection [95]. Therefore, already within legumes the effect of ethylene on nodule formation and rhizobial infection might differ depending on environmental conditions and rhizobial infection strategy.

Ethylene has little effect on root nodule formation by the actinorhizal species $D$. trinervis [96]. Treatment with the ethylene precursor ACC or the ethylene releasing agent 2-chloroethylphosphonic acid (CEPA) only marginally reduced $D$. trinervis nodule number. On the other hand, addition of AVG or silver ions, which block ethylene perception, resulted in slightly more nodules in response to Frankia inoculation [96]. Despite a similar trend, these effects are much less pronounced than observed in legumes. Additionally, $D$. trinervis root growth is strongly affected by interference with ethylene homeostasis [96]. Thus, it cannot be excluded that a small change in nodule number results from an indirect effect of ethylene on root morphology. Therefore, a role for ethylene in regulation of the $D$. trinervisFrankia symbiosis is not supported.

In Parasponia, ethylene signaling is required for efficient intracellular infection by rhizobium [Chapter 5]. Rhizobia infect Parasponia intercellularly and form small apoplastic colonies in between nodule cells $[97,98]$. From there, cell wall-bound infection threads are launched. Once inside the cell, these threads change morphology and ramify to form fixation threads [97,99]. P. andersonii plants mutated in EIN2 are ethylene insensitive and develop a wildtype number of nodules [Chapter 5], indicating that in Parasponia ethylene does not 
regulate nodule number. However, Panein2 mutant nodules are smaller than wild type and are affected in fixation thread formation [Chapter 5]. In wild-type nodules, several cell layers containing infection threads are found just below the nodule meristem. These are followed by one or two cell layers in which infected cells increase in size and fragmentation of the vacuole occurs, which coincides with the start of fixation thread formation [97]. Immediately below these cell layers, infected cells are fully filled with fixation threads. Some Panein2 mutant nodules contain only infection threads as well as enlarged apoplastic colonies, but no fixation threads [Chapter 5]. Others contain fixation threads but fixation thread formation appears less efficient compared to wild type. Multiple cell layers containing infection threads are present and infected cells are interspersed with cells that contain only few fixation threads and still display vacuole fragmentation [Chapter 5]. In legumes, intracellular infection also involves cell enlargement, which results from endoreduplication, a prerequisite for intracellular infection [100]. Research on arabidopsis has revealed that ethylene inhibits cell proliferation in the root apical meristem through induction of endoreduplication [101]. Consistently, ethylene has been shown to promote endoreduplication in arabidopsis and cucumber (Cucumis sativus) hypocotyl cells [102,103]. Therefore, it could be hypothesized that inefficient formation of fixation threads in Panein2 mutant nodules is due to the absence of ethylene-induced endoreduplication. In legumes, ethylene is likely not required to perform such function, as Mtein2 nodules are properly infected by rhizobium bacteria [31]. This indicates a major difference between legumes and Parasponia and suggests that ethylene function was independently recruited to regulate rhizobium symbiosis [Chapter 5]. Overall, it can be concluded that ethylene performs distinct functions during the nitrogenfixing symbioses of legumes, actinorhizas and Parasponia.

\section{Strigolactones}

The GRAS-type transcriptional regulators NSP1 and NSP2 are essential for nodule formation by legumes and Parasponia $[67,104,105]$ [Chapter 5]. In addition, both proteins are required for basal expression of the strigolactone biosynthesis gene $D 27$ in medicago, rice and Parasponia [106] [Chapter 5]. In medicago, MtD27 expression is strongly induced following rhizobial LCO perception in an MtNSP1- and MtNSP2-dependent manner [107] [Chapter 4]. During rhizobial infection, $M t D 27$ expression is associated with growing infection threads and dividing cells of the nodule primordium. At later stages, MtD27 expression is restricted to the meristem and distal infection zone of mature nodules [107] [Chapter 4]. A similar expression pattern is observed for the carotenoid cleavage dioxygenases MtCCD7 and MtCCD8 that function downstream of MtD27 [48,107] [Chapter 4]. This suggests a role for strigolactones in the legume-rhizobium symbiosis. Strigolactones, however, are not essential for legume root nodule formation. Pea (Pisum sativum) and lotus strigolactone-deficient mutants 
revealed only marginal reduction in nodule numbers [108-110]. Similarly, RNAi-mediated knockdown of MtD27 expression did not affect nodule number nor intracellular infection by rhizobium bacteria [107] [Chapter 4]. In Parasponia, strigolactone function has not been studied. However, expression patterns of PanD27, PanCCD7 and PanCCD8 suggest that also for Parasponia nodule formation strigolactones are not essential. Whereas expression of PanD27 and PanCCD7 is enhanced in Parasponia nodules, expression of PanCCD8 is strongly reduced (Figure 1). Therefore, NSP1 and NSP2 are likely required to regulate genes other than $D 27$ during nitrogen-fixing symbioses.

\section{Genetic Adaptations in Hormonal Networks}

As outlined above, plant hormones perform essential symbiotic functions. Therefore, nodulating lineages might have acquired genetic adaptations in hormonal networks to support nodule formation. However, little evidence for such adaptations currently exists in literature. One study reports identification of a legume-specific duplication in type-A Response Regulators [56]. These were identified based on a phylogenetic strategy to identify duplicate gene pairs that were retained in the legume Papilionoideae subfamily. This subfamily experienced a whole-genome duplication approximately 58 million years ago, shortly after emergence of the legume-rhizobium symbiosis [56,111,112]. One orthology group containing retained gene duplicates harbors the medicago type-A Response Regulators MtRR9 and MtRR11. Both genes are induced within hours of rhizobium LCO application [56]. This induction is dependent on the cytokinin receptor MTCRE1, suggesting it results from LCO-induced cytokinin signaling [20] [Chapter 3]. RNAi-mediated knockdown of MtRR9 expression equally affected both lateral root and nodule development [56]. Therefore, it is questionable whether the MtRR9-MtRR11 duplication was specifically retained to support papilionoid nodule formation.

Despite performing an essential role in nodule initiation, the cytokinin receptors LjLHK1 and MtCRE1 most likely do not contain genetic adaptations. Trans-complementation using an orthologous receptor from arabidopsis (Arabidopsis thaliana) fully restored nodule organogenesis on Ljlhk1 and Mtcre1 mutant roots [60,61]. These experiments were performed using an AtAHK4/AtCRE1 genomic clone, suggesting that neither the regulatory sequences nor the protein sequences of LjLHK1 and MtCRE1 contain specific adaptations that are essential for nodule formation. In this respect, it is interesting that PanHK4 is not required for root nodule formation by $P$. andersonii [Chapter 5]. As $P$. andersonii is more closely related to legumes than arabidopsis, it is likely that ectopic expression of PanHK4 would also restore nodulation on Mtcre1 or Ljlhk1 mutant roots. This suggests that legumes 

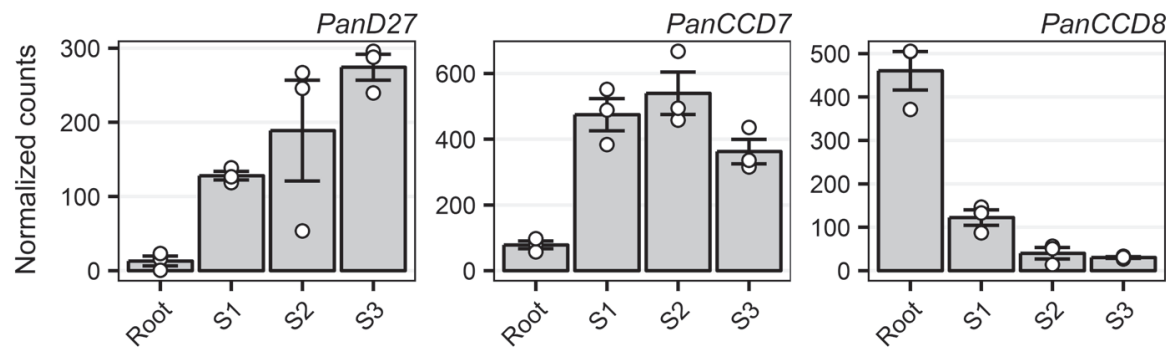

Figure 1. Expression of PanD27, PanCCD7 and PanCCD8 in P. andersonii roots and nodules.

Expression of PanD27, PanCCD7 and PanCCD8 in roots and during three stages of nodule development (S1-S3). Expression levels are presented as DESeq2 normalized read counts. Data represent means of three biological replicates \pm SEM. Dots represent expression values in each of the biological replicates. Data were obtained from van Velzen et al. [64].

and Parasponia have different dependencies on cytokinin signaling or specifically MtCRE1/ LjLHK1/PanHK4 function during nodule initiation.

In medicago and lotus, MtCRE1 and LjLHK1 are the most highly expressed cytokinin receptor genes in roots, respectively $[60,61]$. Consistently, only $L j l h k 1$ mutants are resistant to the effect of exogenous cytokinin on root growth, indicating that LjLHK1 is the main sensor of exogenous cytokinin in lotus roots [60]. In contrast, in P. andersonii, PanHK3 is highest expressed cytokinin receptor gene in roots (Figure 2). Overexpression of the lotus orthologue of PanHK3 is sufficient to rescue the nodulation defect of the Ljlhk1 mutant [60]. This suggests that a difference in relative expression of cytokinin receptor genes in roots could explain the difference in symbiotic dependencies on MtCRE1/LjLHK1/PanHK4 between legumes and Parasponia. Alternatively, Parasponia root nodule formation might not require cytokinin signaling. An important regulator of root nodule formation in legumes, and most probably also Parasponia, is the transcription factor NIN [64,113-115]. In legumes, NIN expression is first induced in the root epidermis in response to perception of rhizobium LCOs [53,114,116]. At subsequent stages, NIN is expressed in the root cortex at sites corresponding to incipient nodule primordia $[53,67,114]$. Induction of NIN in the root cortex requires MtCRE1/LjLHK1dependent cytokinin signaling [53,55,59]. In medicago, ectopic expression of MtNIN in the root cortex can induce nodule organogenesis in an MtCRE1-independent manner [53]. This suggests, that in legumes, an important function of cytokinin signaling is cortical activation of NIN expression. In Parasponia, cell divisions associated with nodule organogenesis are first induced in the root epidermis $[15,30,98]$. This might result from direct activation of PanNIN by rhizobium LCO-induced signaling. Therefore, it is possible that Parasponia root nodule formation is independent of cytokinin signaling. 
To identify genetic adaptations that correlate with nodulation capacity, studies on closely related species that differ in symbiotic capacity are most helpful. The Parasponia lineage is embedded in the non-nodulating Trema genus [64,117]. Genome comparisons between both groups of species identified eleven copy number variants among $\sim 1,800$ putative symbiotic genes [64]. This included one gene involved in hormone homeostasis: IPT4, an isopentenyl transferase involved in cytokinin biosynthesis that has been lost from the genomes of Parasponia spp. [64]. Cytokinin is a positive regulator of legume nodule formation [118] and therefore it is difficult to envision how loss of this gene might promote Parasponia-rhizobium symbiosis.

Transcriptome comparisons between non-inoculated roots of two Parasponia and three Trema species indicated transcriptional divergence between Parasponia and Trema species. Among the genes differentially expressed between Parasponia and Trema roots are several involved in jasmonic acid biosynthesis [Chapter 6]. The jasmonic acid precursor cis-(+)12-oxophytodienoic acid (OPDA) is synthesized from $\alpha$-linolenic acid through consecutive activity of 13S-lipoxynases (LOX), allene oxide synthases (AOS) and allene oxide cyclases (AOC) $[119,120]$. Two LOX- and one AOC-encoding gene(s) are consistently higher expressed in roots of Parasponia compared to Trema [Chapter 6]. This coincides with increased concentrations of OPDA and jasmonic acid, but not its bio-active conjugate JA-Ile, in roots of Parasponia. Conversely salicylic acid concentrations are higher in roots of Trema, indicating a shift in the jasmonic acid/salicylic acid balance between Parasponia and Trema roots [Chapter 6]. However, this balance shift might not be correlated to nodulation capacity as $P$. andersonii mutants in jasmonic acid biosynthesis are not affected in nodule development [Chapter 6]. How this affected salicylic acid concentrations remains undetermined.

In the legumes alfalfa and lotus, expression of jasmonic acid biosynthesis genes is induced during the first twelve hours after inoculation with compatible rhizobium bacteria [121,122]. Additionally, several studies report an accumulation of LOX transcripts and LOX protein in legume root nodules [123-125]. However, measurements in medicago, C. glauca and D. glomerata roots and nodules indicate similar jasmonic acid concentrations between both organs [126]. Interference with jasmonic acid biosynthesis through RNAi-mediated silencing of multiple LOX genes in soybean or MtAOC1 in medicago did neither disturb nodule development nor nodule functioning [126,127], in line with results in Parasponia [Chapter 6]. Similarly, enzymatic jasmonic acid biosynthesis also appears not to be required for mycorrhizal symbiosis in rice (Oryza sativa), tomato (Solanum lycopersicum) and $M$. truncatula [128-130]. 


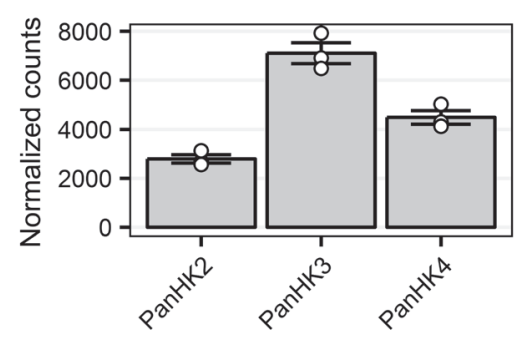

Figure 2. Expression of PanHK2, PanHK3 and PanHK4 in roots of $P$. andersonii.

Expression of PanHK2, PanHK3 and PanHK4 in young $P$. andersonii root zones, excluding the root tip. Expression levels are presented as DESeq2 normalized read counts. Data represent means of three biological replicates \pm SEM. Dots represent expression values in each of the biological replicates. Data were obtained from Chapter 6.

In the near future, efforts of large international consortia might uncover genetic adaptations that correlate with nodulation capacity. These consortia aim to sequence the genomes of multiple pairs of closely related nodulating and non-nodulating species, including actinorhizas. This facilitates comparisons, as done for Parasponia-Trema, to be performed on a much larger scale. However, the success of these studies highly depends on the evolutionary history of the nodulation trait. Data suggest the possibility that Trema and related Rosales species lost nodulation ability in recent history [64]. If this scenario would apply to the entire nitrogen-fixation clade, identification of genetic adaptations that correlate with nodulation ability would be challenging. Therefore, it remains to be seen whether adaptations in hormonal networks that are essential for nitrogen-fixing nodule symbioses will be found.

\section{Concluding Remarks}

It is evident that hormones are important regulators of nitrogen-fixing nodule symbioses. However, to what extent hormone function during nodule formation is conserved between different nodulating lineages is still far from clear. To increase insight, comparative studies are essential. These can include reverse genetic studies to determine the role of certain hormonal pathways in nodule formation in legumes, Parasponia and actinorhiza or trans-complementation assays to determine whether symbiotic genes contain specific adaptations. In this respect, the stable transformation and CRISPR/Cas9 genome-editing protocol developed for $P$. andersonii provides a valuable tool [Chapter 5]. Development of similar protocols for one or several actinorhizal species would allow comparative analyses to be performed. This will greatly enhance our understanding of hormone function during nodule development in different nitrogen-fixing lineages. 


\section{ACKNOWLEDGEMENTS}

\section{The author would like to thank Titis Wardhani for sharing data prior to publication.}

\section{REFERENCES}

Bulgarelli, D. et al. (2013) Structure and functions of the bacterial microbiota of plants. Annu. Rev. Plant Biol. $64,807-838$

2 Oldroyd, G.E.D. (2013) Speak, friend, and enter: signalling systems that promote beneficial symbiotic associations in plants. Nat. Rev. Microbiol. 11, 252-263

3 Doyle, J.J. (2011) Phylogenetic perspectives on the origins of nodulation. Mol. Plant-Microbe Interact. 24, 1289-1295

4 Li, H.-L. et al. (2015) Large-scale phylogenetic analyses reveal multiple gains of actinorhizal nitrogen-fixing symbioses in angiosperms associated with climate change. Sci. Rep. 5, 14023

5 Soltis, D.E. et al. (1995) Chloroplast gene sequence data suggest a single origin of the predisposition for symbiotic nitrogen fixation in angiosperms. Proc. Natl. Acad. Sci. U. S. A. 92, 2647-2651

6 Werner, G.D.A. et al. (2014) A single evolutionary innovation drives the deep evolution of symbiotic $\mathrm{N}_{2}-$ fixation in angiosperms. Nat. Commun. 5, 4087

7 Remigi, P. et al. (2016) Symbiosis within symbiosis: evolving nitrogen-fixing legume symbionts. Trends Microbiol. 24, 63-75

8 Wall, L.G. (2000) The actinorhizal symbiosis. J. Plant Growth Regul. 19, 167-182

9 Santi, C. et al. (2013) Biological nitrogen fixation in non-legume plants. Ann. Bot. 111, 743-767

10 Geurts, R. et al. (2012) Exploiting an ancient signalling machinery to enjoy a nitrogen fixing symbiosis. Curr. Opin. Plant Biol. 15, 438-443

11 Delaux, P.M. et al. (2015) Tracing the evolutionary path to nitrogen-fixing crops. Curr. Opin. Plant Biol. 26, 95-99

12 Martin, F.M. et al. (2017) Ancestral alliances: Plant mutualistic symbioses with fungi and bacteria. Science 356, eaad4501

13 Parniske, M. (2008) Arbuscular mycorrhiza: the mother of plant root endosymbioses. Nat. Rev. Microbiol. 6, 763-775

14 Pawlowski, K. and Demchenko, K.N. (2012) The diversity of actinorhizal symbiosis. Protoplasma 249, 967979

15 Geurts, R. et al. (2016) What does it take to evolve a nitrogen-fixing endosymbiosis? Trends Plant Sci. 21, 199-208

16 Santner, A. and Estelle, M. (2009) Recent advances and emerging trends in plant hormone signalling. Nature 459, 1071-1078

17 Durbak, A. et al. (2012) Hormone signaling in plant development. Curr. Opin. Plant Biol. 15, 92-96

18 Larrainzar, E. et al. (2015) Deep sequencing of the Medicago truncatula root transcriptome reveals a massive and early interaction between Nod factor and ethylene signals. Plant Physiol. 169, 233-265

19 Jardinaud, M.-F. et al. (2016) A laser dissection-RNAseq analysis highlights the activation of cytokinin pathways by Nod factors in the Medicago truncatula root epidermis. Plant Physiol. 171, 2256-2276

20 van Zeijl, A. et al. (2015) Rhizobium lipo-chitooligosaccharide signaling triggers accumulation of cytokinins in Medicago truncatula roots. Mol. Plant 8, 1213-1226

21 Ferguson, B.J. and Mathesius, U. (2014) Phytohormone regulation of legume-rhizobia interactions. J. Chem. Ecol. 40, 770-790

22 Ryu, H. et al. (2012) Plant hormonal regulation of nitrogen-fixing nodule organogenesis. Mol. Cells 34, 117126

23 Benkova, E. et al. (2003) Local, efflux-dependent auxin gradients as a common module for plant organ formation. Cell 115, 591-602

24 Blilou, I. et al. (2005) The PIN auxin efflux facilitator network controls growth and patterning in Arabidopsis 
roots. Nature 433, 39-44

Turner, M. et al. (2013) Ectopic expression of miR160 results in auxin hypersensitivity, cytokinin hyposensitivity, and inhibition of symbiotic nodule development in soybean. Plant Physiol. 162, 2042-2055

26 Suzaki, T. et al. (2012) Positive and negative regulation of cortical cell division during root nodule development in Lotus japonicus is accompanied by auxin response. Development 139, 3997-4006

27 Mathesius, U. et al. (1998) Auxin transport inhibition precedes root nodule formation in white clover roots and is regulated by flavonoids and derivatives of chitin oligosaccharides. Plant J. 14, 23-34

28 Huo, X. et al. (2006) RNAi phenotypes and the localization of a protein::GUS fusion imply a role for Medicago truncatula PIN genes in nodulation. J. Plant Growth Regul. 25, 156-165

29 Imanishi, L. et al. (2014) Role of auxin during intercellular infection of Discaria trinervis by Frankia. Front. Plant Sci. 5, 399

30 Xiao, T.T. (2015) Root and Nodule. Lateral organ development in $\mathrm{N}_{2}$-fixing plants. Wageningen PhD thesis. ISBN: 9789462572768

31 Xiao, T.T. et al. (2014) Fate map of Medicago truncatula root nodules. Development 141, 3517-3528

32 Deinum, E.E. et al. (2012) Modeling a cortical auxin maximum for nodulation: different signatures of potential strategies. Front. Plant Sci. 3, 96

33 Deinum, E.E. et al. (2016) Quantitative modelling of legume root nodule primordium induction by a diffusive signal of epidermal origin that inhibits auxin efflux. BMC Plant Biol. 16, 254

34 Wasson, A.P. et al. (2006) Silencing the flavonoid pathway in Medicago truncatula inhibits root nodule formation and prevents auxin transport regulation by rhizobia. Plant Cell 18, 1617-1629

35 Fang, Y. and Hirsch, A.M. (1998) Studying early nodulin gene ENOD40 expression and induction by nodulation factor and cytokinin in transgenic alfalfa. Plant Physiol. 116, 53-68

36 Hirsch, A.M. et al. (1989) Early nodulin genes are induced in alfalfa root outgrowths elicited by auxin transport inhibitors. Proc. Natl. Acad. Sci. U. S. A. 86, 1244-1248

37 Rightmyer, A.P. and Long, S.R. (2011) Pseudonodule formation by wild-type and symbiotic mutant Medicago truncatula in response to auxin transport inhibitors. Mol. Plant-Microbe Interact. 24, 1372-1384

38 Scheres, B. et al. (1992) The PSENOD12 gene is expressed at 2 different sites in Afghanistan pea pseudonodules induced by auxin transport inhibitors. Plant Physiol. 100, 1649-1655

39 Wu, C. et al. (1996) The auxin transport inhibitor N-(1-naphthyl)phthalamic acid elicits pseudonodules on nonnodulating mutants of white sweetclover. Plant Physiol. 110, 501-510

$40 \mathrm{Ng}$, J.L.P. et al. (2015) Flavonoids and auxin transport inhibitors rescue symbiotic nodulation in the Medicago truncatula cytokinin perception mutant cre1. Plant Cell 27, 2210-2226

41 Peer, W.A. and Murphy, A.S. (2007) Flavonoids and auxin transport: modulators or regulators? Trends Plant Sci. $12,556-563$

42 Abdel-Lateif, K. et al. (2013) Silencing of the chalcone synthase gene in Casuarina glauca highlights the important role of flavonoids during nodulation. New Phytol. 199, 1012-1021

43 Abdel-Lateif, K. et al. (2012) The role of flavonoids in the establishment of plant roots endosymbioses with arbuscular mycorrhiza fungi, rhizobia and Frankia bacteria. Plant Signal. Behav. 7, 636-641

44 Kramer, E.M. (2004) PIN and AUX/LAX proteins: Their role in auxin accumulation. Trends Plant Sci. 9, 578-582

45 Delbarre, A. et al. (1996) Comparison of mechanisms controlling uptake and accumulation of 2,4-dichlorophenoxy acetic acid, naphthalene-1-acetic acid, and indole-3-acetic acid in suspension-cultured tobacco cells. Planta 198, 532-541

46 Roy, S. et al. (2017) MtLAX2, a functional homologue of the auxin importer AtAUX1, is required for nodule organogenesis. Plant Physiol. 174, 326-338

47 Péret, B. et al. (2007) Auxin influx activity is associated with Frankia infection during actinorhizal nodule formation in Casuarina glauca. Plant Physiol. 144, 1852-1862

48 Breakspear, A. et al. (2014) The root hair "infectome" of Medicago truncatula uncovers changes in cell cycle genes and reveals a requirement for auxin signaling in rhizobial infection. Plant Cell 26, 4680-4701

49 Liu, C.-W. et al. (2015) Cytokinin responses counterpoint auxin signaling during rhizobial infection. Plant Signal. Behav. 10, e1019982

50 Berry, A.M. et al. (1993) Hopanoid lipids compose the Frankia vesicle envelope, presumptive barrier of oxygen diffusion to nitrogenase. Proc. Natl. Acad. Sci. U. S. A. 90, 6091-6094

51 Hammad, Y. et al. (2003) A possible role for phenyl acetic acid (PAA) on Alnus glutinosa nodulation by Frankia. 
Plant Soil 254, 193-205

52 Etemadi, M. et al. (2014) Auxin perception is required for arbuscule development in arbuscular mycorrhizal symbiosis. Plant Physiol. 166, 281-292

53 Vernié, T. et al. (2015) The NIN transcription factor coordinates diverse nodulation programs in different tissues of the Medicago truncatula root. Plant Cell 27, 3410-3424

54 Vernié, T. et al. (2008) EFD is an ERF transcription factor involved in the control of nodule number and differentiation in Medicago truncatula. Plant Cell 20, 2696-2713

55 Plet, J. et al. (2011) MtCRE1-dependent cytokinin signaling integrates bacterial and plant cues to coordinate symbiotic nodule organogenesis in Medicago truncatula. Plant J. 65, 622-633

56 Op den Camp, R.H.M. et al. (2011) A phylogenetic strategy based on a legume-specific whole genome duplication yields symbiotic cytokinin Type-A Response Regulators. Plant Physiol. 157, 2013-2022

57 Gonzalez-Rizzo, S. et al. (2006) The Medicago truncatula CRE1 cytokinin receptor regulates lateral root development and early symbiotic interaction with Sinorhizobium meliloti. Plant Cell 18, 2680-2693

58 Reid, D.E. et al. (2016) CYTOKININ OXIDASE/DEHYDROGENASE3 maintains cytokinin homeostasis during root and nodule development in Lotus japonicus. Plant Physiol. 170, 1060-1074

59 Murray, J.D. et al. (2007) A cytokinin perception mutant colonized by Rhizobium in the absence of nodule organogenesis. Science 315, 101-104

60 Held, M. et al. (2014) Lotus japonicus cytokinin receptors work partially redundantly to mediate nodule formation. Plant Cell 26, 678-694

61 Boivin, S. et al. (2016) Different cytokinin histidine kinase receptors regulate nodule initiation as well as later nodule developmental stages in Medicago truncatula. Plant, Cell Environ. 39, 2198-2209

62 Arrighi, J.-F. et al. (2012) Aeschynomene evenia, a model plant for studying the molecular genetics of the nod-independent rhizobium-legume symbiosis. Mol. Plant-Microbe Interact. 25, 851-861

63 Fabre, S. et al. (2015) The Nod factor-independent nodulation in Aeschynomene evenia required the common plant-microbe symbiotic “toolkit." Plant Physiol. 169, 2654-2664

64 van Velzen, R. et al. (2017) Parallel loss of symbiosis genes in relatives of nitrogen-fixing non-legume Parasponia. bioRxiv DOI: 10.1101/169706

65 Torrey, J.G. (1961) Kinetin as trigger for mitosis in mature endomitotic plant cells. Exp. Cell Res. 23, 281-299

66 Mathesius, U. et al. (2000) Temporal and spatial order of events during the induction of cortical cell divisions in white clover by Rhizobium leguminosarum bv. trifolii inoculation or localized cytokinin addition. Mol. Plant-Microbe Interact. 13, 617-628

67 Heckmann, A.B. et al. (2011) Cytokinin induction of root nodule primordia in Lotus japonicus is regulated by a mechanism operating in the root cortex. Mol. Plant-Microbe Interact. 24, 1385-1395

68 Cooper, J. and Long, S. (1994) Morphogenetic rescue of Rhizobium meliloti nodulation mutants by transZeatin secretion. Plant Cell 6, 215-225

69 Rodriguez-Barrueco, C. and Bermudez de Castro, F. (1973) Cytokinin-induced pseudonodules on Alnus glutinosa. Physiol. Plant. 29, 277-280

70 Guinel, F.C. (2015) Ethylene, a hormone at the center-stage of nodulation. Front. Plant Sci. 6, 1121

71 Penmetsa, R.V. and Cook, D. (1997) A legume ethylene-insensitive mutant hyperinfected by its rhizobial symbiont. Science $275,527-530$

72 Nukui, N. et al. (2000) Effects of ethylene precursor and inhibitors for ethylene biosynthesis and perception on nodulation in Lotus japonicus and Macroptilium atropurpureum. Plant Cell Physiol. 41, 893-897

73 Oldroyd, G.E.D. et al. (2001) Ethylene inhibits the Nod factor signal transduction pathway of Medicago truncatula. Plant Cell 13, 1835-1849

74 Zaat, S.A.J. et al. (1989) The ethylene-inhibitor aminoethoxyvinylglycine restores normal nodulation by Rhizobium leguminosarum biovar. viciae on Vicia sativa subsp. nigra by suppressing the "Thick and short roots" phenotype. Planta 177, 141-150

75 Schmidt, J.S. et al. (1999) Regulation of soybean nodulation independent of ethylene signaling. Plant Physiol. 119, 951-960

76 Lee, K.H. and Larue, T. (1992) Exogenous ethylene inhibits nodulation of Pisum sativum L. cv Sparkle. Plant Physiol. 100, 1759-1763

77 Peters, N.K. and Crist-Estes, D.K. (1989) Nodule formation is stimulated by the ethylene inhibitor aminoethoxyvinylglycine. Plant Physiol. 91, 690-693 
78 Alonso, J.M. et al. (1999) EIN2, a bifunctional transducer of ethylene and stress responses in Arabidopsis. Science 284, 2148-2152

79 Penmetsa, R. V et al. (2008) The Medicago truncatula ortholog of Arabidopsis EIN2, sickle, is a negative regulator of symbiotic and pathogenic microbial associations. Plant J. 55, 580-595

80 Nukui, N. et al. (2004) Transgenic Lotus japonicus with an ethylene receptor gene Cm-ERS1/H70A enhances formation of infection threads and nodule primordia. Plant Cell Physiol. 45, 427-435

81 Lohar, D. et al. (2009) Ethylene insensitivity conferred by a mutated Arabidopsis ethylene receptor gene alters nodulation in transgenic Lotus japonicus. Ann. Bot. 104, 277-285

82 Miyata, K. et al. (2013) Two distinct EIN2 genes cooperatively regulate ethylene signaling in Lotus japonicus. Plant Cell Physiol. 54, 1469-1477

83 van Workum, W.A. et al. (1995) Ethylene prevents nodulation of Vicia sativa ssp. nigra by exopolysaccharidedeficient mutants of Rhizobium leguminosarum bv. viciae. Mol. Plant-Microbe Interact. 8, 278-285

84 Suganuma, N. et al. (1995) Enhanced production of ethylene by soybean roots after inoculation with Bradyrhizobium japonicum. Plant Sci. 111, 163-168

85 Ligero, F. et al. (1987) Evolution of ethylene from roots and nodulation rate of Alfalfa (Medicago sativa L.) plants inoculated with Rhizobium meliloti as affected by the presence of nitrate. J. Plant Physiol. 129, 461467

86 Xie, Z.-P. et al. (1996) Ethylene responsiveness of soybean cultivars characterized by leaf senescence, chitinase induction and nodulation. J. Plant Physiol. 149, 690-694

87 Capoen, W. et al. (2010) Sesbania rostrata: a case study of natural variation in legume nodulation. New Phytol. 186, 340-345

88 Goormachtig, S. et al. (2004) Switch from intracellular to intercellular invasion during water stress-tolerant legume nodulation. Proc. Natl. Acad. Sci. U. S. A. 101, 6303-6308

89 D'Haeze, W. et al. (2003) Reactive oxygen species and ethylene play a positive role in lateral root base nodulation of a semiaquatic legume. Proc. Natl. Acad. Sci. U. S. A. 100, 11789-11794

90 Ndoye, I. et al. (1994) Root nodulation of Sesbania rostrata. J. Bacteriol. 176, 1060-1068

91 Goormachtig, S. et al. (1998) The symbiotic interaction between Azorhizobium caulinodans and Sesbania rostrata. In Plant-Microbe Interactions (Biswas, B. B. and Das, H. K., eds), pp. 117-164, Springer US

92 Lievens, S. et al. (2005) Gibberellins are involved in nodulation of Sesbania rostrata. Plant Physiol. 139, 13661379

93 D'Haeze, W. et al. (1998) Roles for Azorhizobial Nod Factors and surface polysaccharides in intercellular invasion and nodule penetration, respectively. Mol. Plant-Microbe Interact. 11, 999-1008

94 Capoen, W. et al. (2009) Calcium spiking patterns and the role of the Calcium/Calmodulin-Dependent Kinase CCaMK in lateral root base nodulation of Sesbania rostrata. Plant Cell 21, 1526-1540

95 Groth, M. et al. (2010) NENA, a Lotus japonicus homolog of Sec13, is required for rhizodermal infection by arbuscular mycorrhiza fungi and rhizobia but dispensable for cortical endosymbiotic development. Plant Cell 22, 2509-2526

96 Valverde, C. and Wall, L.G. (2005) Ethylene modulates the susceptibility of the root for nodulation in actinorhizal Discaria trinervis. Physiol. Plant. 124, 121-131

97 Op den Camp, R.H.M. et al. (2012) Nonlegume Parasponia andersonii deploys a broad rhizobium host range strategy resulting in largely variable symbiotic effectiveness. Mol. Plant-Microbe Interact. 25, 954-963

98 Lancelle, S.A. and Torrey, J.G. (1984) Early development of Rhizobium-induced root nodules of Parasponia rigida. I. Infection and early nodule initiation. Protoplasma 123, 26-37

99 Price, G.D. et al. (1984) Structure of nodules formed by Rhizobium strain ANU289 in the nonlegume Parasponia and the legume siratro (Macroptilium atropurpureum). Bot. Gaz. 145, 444-451

100 Vinardell, J.M. et al. (2003) Endoreduplication mediated by the anaphase-promoting complex activator CCS52A is required for symbiotic cell differentiation in Medicago truncatula nodules. Plant Cell 15, 20932105

101 Street, I.H. et al. (2015) Ethylene inhibits cell proliferation of the Arabidopsis root meristem. Plant Physiol. 169, 338-350

102 Gendreau, E. et al. (1999) Gibberellin and ethylene control endoreduplication levels in the Arabidopsis thaliana hypocotyl. Planta 209, 513-516

103 Dan, H. et al. (2003) Ethylene stimulates endoreduplication but inhibits cytokinesis in cucumber hypocotyl 
epidermis. Plant Physiol. 133, 1726-1731

104 Smit, P. et al. (2005) NSP1 of the GRAS protein family is essential for rhizobial Nod factor-induced transcription. Science 308, 1789-1791

105 Kalo, P. et al. (2005) Nodulation signaling in legumes requires NSP2, a member of the GRAS family of transcriptional regulators. Science 308, 1786-1789

106 Liu, W. et al. (2011) Strigolactone biosynthesis in Medicago truncatula and rice requires the symbiotic GRAStype transcription factors NSP1 and NSP2. Plant Cell 23, 3853-3865

107 van Zeijl, A. et al. (2015) The strigolactone biosynthesis gene DWARF27 is co-opted in rhizobium symbiosis. BMC Plant Biol. 15, 260

108 Liu, J. et al. (2013) Carotenoid cleavage dioxygenase 7 modulates plant growth, reproduction, senescence, and determinate nodulation in the model legume Lotus japonicus. J. Exp. Bot. 64, 1967-1981

109 Foo, E. and Davies, N.W. (2011) Strigolactones promote nodulation in pea. Planta 234, 1073-1081

110 Foo, E. et al. (2013) Strigolactones and the regulation of pea symbioses in response to nitrate and phosphate deficiency. Mol. Plant 6, 76-87

111 Pfeil, B. et al. (2005) Placing paleopolyploidy in relation to taxon divergence: a phylogenetic analysis in legumes using 39 gene families. Syst. Biol. 54, 441-454

112 Cannon, S.B. et al. (2010) Polyploidy did not predate the evolution of nodulation in all legumes. PLoS One 5, e11630

113 Marsh, J.F. et al. (2007) Medicago truncatula NIN is essential for rhizobial-independent nodule organogenesis induced by autoactive calcium/calmodulin-dependent protein kinase. Plant Physiol. 144, 324-335

114 Schauser, L. et al. (1999) A plant regulator controlling development of symbiotic root nodules. Nature 402, 191-195

115 Soyano, T. et al. (2013) NODULE INCEPTION directly targets NF-Y subunit genes to regulate essential processes of root nodule development in Lotus japonicus. PLoS Genet. 9, e1003352

116 Madsen, L.H. et al. (2010) The molecular network governing nodule organogenesis and infection in the model legume Lotus japonicus. Nat. Commun. 1, 10

117 Yang, M. et al. (2013) Molecular phylogenetics and character evolution of Cannabaceae. Taxon 62, 473-485

118 Frugier, F. et al. (2008) Cytokinin: secret agent of symbiosis. Trends Plant Sci. 13, 115-120

119 Wasternack, C. and Hause, B. (2013) Jasmonates: Biosynthesis, perception, signal transduction and action in plant stress response, growth and development. An update to the 2007 review in Annals of Botany. Ann. Bot. 111, 1021-1058

120 Wasternack, C. (2015) How jasmonates earned their laurels: past and present. J. Plant Growth Regul. 34, 761-794

121 Kouchi, H. et al. (2004) Large-scale analysis of gene expression profiles during early stages of root nodule formation in a model legume, Lotus japonicus. DNA Res. 11, 263-274

122 Bueno, P. et al. (2001) Time-course of lipoxygenase, antioxidant enzyme activities and $\mathrm{H}_{2} \mathrm{O}_{2}$ accumulation during the early stages of Rhizobium-legume symbiosis. New Phytol. 152, 91-96

123 Perlick, A.M. et al. (1996) The Vicia faba lipoxygenase gene VfLOX1 is expressed in the root nodule parenchyma. Mol. Plant-Microbe Interact. 9, 860-863

124 Porta, H. et al. (1999) Analysis of lipoxygenase mRNA accumulation in the common bean (Phaseolus vulgaris L.) during development and under stress conditions. Plant Cell Physiol. 40, 850-858

125 Wisniewski, J.P. et al. (1999) Isolation of lipoxygenase cDNA clones from pea nodule mRNA. Plant Mol. Biol. 39, 775-783

126 Zdyb, A. et al. (2011) Jasmonate biosynthesis in legume and actinorhizal nodules. New Phytol. 189, 568-579

127 Hayashi, S. et al. (2008) Molecular analysis of lipoxygenases associated with nodule development in soybean. Mol. Plant-Microbe Interact. 21, 843-853

128 Tejeda-Sartorius, M. et al. (2008) Jasmonic acid influences mycorrhizal colonization in tomato plants by modifying the expression of genes involved in carbohydrate partitioning. Physiol. Plant. 133, 339-353

129 Isayenkov, S. et al. (2005) Suppression of allene oxide cyclase in hairy roots of Medicago truncatula reduces jasmonate levels and the degree of mycorrhization with Glomus intraradices. Plant Physiol. 139, 1401-1410

130 Gutjahr, C. et al. (2015) Full establishment of arbuscular mycorrhizal symbiosis in rice occurs independently of enzymatic jasmonate biosynthesis. PLoS One 10, e0123422 


\section{SUMMARY}

Nitrogen is a key element for plant growth. To meet nitrogen demands, some plants establish an endosymbiotic relationship with nitrogen-fixing rhizobium or Frankia bacteria. This involves formation of specialized root lateral organs, named nodules. These nodules are colonized intracellularly, which creates optimal physiological conditions for the fixation of atmospheric nitrogen by the microbial symbiont. Nitrogen-fixing endosymbioses are found among four related taxonomic orders that together form the nitrogen-fixation clade. Within this clade, nodulation is restricted to ten separate lineages that are scattered among mostly non-nodulating plant species. This limited distribution suggests that genetic adaptations that allowed nodulation to evolve occurred in a common ancestor.

A major aim of the scientific community is to unravel the evolutionary trajectory towards a nitrogen-fixing nodule symbiosis. The formation of nitrogen-fixing root nodules is best studied in legumes (Fabaceae, order Fabales); especially in Lotus japonicus and Medicago truncatula, two species that serve as model. Legumes and Parasponia (Cannabaceae, order Rosales) represent the only two lineages that can form nodules with rhizobium bacteria. Studies on M. truncatula, L. japonicus and Parasponia showed, amongst others, that nodule formation is initiated upon perception of rhizobial secreted lipo-chitooligosaccharide (LCO) signals. These signals are structurally related to the symbiotic signals produced by arbuscular mycorrhizal fungi. These obligate biotropic fungi colonize roots of most land plants and form dense hyphal structures inside existing root cortical cells.

Rhizobial and mycorrhizal LCOs are perceived by LysM-domain-containing receptor-like kinases. These activate a signaling pathway that is largely shared between both symbioses. Symbiotic LCO receptors are closely related to chitin innate immune receptors, and some receptors even function in symbiotic as well as innate immune signaling. In Chapter 2, I review the intertwining of symbiotic LCO perception and chitin-triggered immunity. Furthermore, I discuss how rhizobia and mycorrhiza might employ LCO signaling to modulate plant immunity. In a perspective, I speculate on a role for plant hormones in immune modulation, besides an important function in nodule organogenesis.

In legumes, nodule organogenesis requires activation of cytokinin signaling. Mutants in the orthologous cytokinin receptor genes MtCRE1 and LjLHK1 in M. truncatula and L. japonicus, respectively, are severely affected in nodule formation. However, how cytokinin signaling is activated in response to rhizobium LCO perception and to what extent this contributes to rhizobium LCO-induced signaling remained elusive. In Chapter 3, I show that the majority of 
transcriptional changes induced in wild-type $M$. truncatula, upon application of rhizobium LCOs, are dependent on activation of MtCRE1-mediated cytokinin signaling. Among the genes induced in wild type are several involved in cytokinin biosynthesis. Consistently, cytokinin measurements indicate that cytokinins rapidly accumulate in $M$. truncatula roots upon treatment with rhizobium LCOs. This includes the bioactive cytokinins isopentenyl adenine and trans-zeatin. Therefore, I argue that cytokinin accumulation represents a key step in the pathway leading to legume root nodule organogenesis.

Strigolactones are plant hormones of which biosynthesis is increased in response to nutrient limitation. In rice (Oryza sativa) and $M$. truncatula, this response requires the GRAS-type transcriptional regulators NSP1 and NSP2. Both proteins regulate expression of DWARF27 (D27), which encodes an enzyme that performs the first committed step in strigolactone biosynthesis. NSP1 and NSP2 are also essential components of the signaling cascade that controls legume root nodule formation. In line with this, I questioned whether the NSP1NSP2-D27 regulatory module functions in rhizobium symbiosis. In Chapter $\mathbf{4}$, I show that in M. truncatula MtD27 expression is induced within hours after treatment with rhizobium LCOs. Spatiotemporal expression studies revealed that MtD27 is expressed in the dividing cells of the nodule primordium. At later stages, its expression becomes confined to the meristem and distal infection zone of the mature nodule. Analysis of the expression pattern of MtCCD7 and MtCCD8, two additional strigolactone biosynthesis genes, showed that these genes are co-expressed with MtD27 in nodule primordia and mature nodules. Additionally, I show that symbiotic expression of MtD27 requires MtNSP1 and MtNSP2. This suggests that the NSP1-NSP2-D27 regulatory module is co-opted in rhizobium symbiosis.

Comparative studies between legumes and nodulating non-legumes could identify shared genetic networks required for nodule formation. We recently adopted Parasponia, the only non-legume lineage able to engage in rhizobium symbiosis. However, to perform functional studies, powerful reverse genetic tools for Parasponia are essential. In Chapter 5, I describe the development of a fast and efficient protocol for CRISPR/Cas9-mediated mutagenesis in Agrobacterium tumefaciens-transformed Parasponia andersonii plants. Using this protocol, stable mutants can be obtained in a period of three months. These mutants can be effectively propagated in vitro, which allows phenotypic evaluation already in the $T_{0}$ generation. As such, phenotypes can be obtained within six months after transformation. As proof-ofprinciple, we mutated PanHK4, PanEIN2, PanNSP1 and PanNSP2. These genes are putatively involved in cytokinin and ethylene signaling and regulation of strigolactone biosynthesis, respectively. Additionally, orthologues of these genes perform essential symbiotic functions in legumes. Panhk4 and Panein2 knockout mutants display developmental phenotypes 
associated with reduced cytokinin and ethylene signaling. Analysis of Pannsp1 and Pannsp2 mutants revealed a conserved role for NSP1 and NSP2 in regulation of the strigolactone biosynthesis genes $D 27$ and MAX1 and root nodule organogenesis. In contrast, symbiotic mutant phenotypes of Panhk4 and Panein2 mutants are different from their legume counterparts. This illustrates the value of Parasponia as comparative model - besides legumes - to study the genetics underlying rhizobium symbiosis.

Phylogenetic reconstruction showed that the Parasponia lineage is embedded in the nonnodulating Trema genus. This close relationship suggests that Parasponia and Trema only recently diverged in nodulation ability. In Chapter 6, I exploited this close relationship to question whether the nodulation trait is associated with gene expression differentiation. To this end, I sequenced root transcriptomes of two Parasponia and three Trema species. Principal component analysis separated all Parasponia samples from those of Trema along the first principal component. This component explains more than half of the observed variance, indicating that the root transcriptomes of two Parasponia species are distinct from that of the Trema sister species T. levigata, as well as the outgroup species T. orientalis and T. tomentosa. To determine, whether the transcriptional differences between Parasponia and Trema are relevant in a symbiotic context, I compared the list of differentially expressed genes to a list of genes that show nodule-enhanced expression in $P$. andersonii. This revealed significant enrichment of nodule-enhanced genes among genes that are lower expressed in roots of Parasponia compared to Trema. Among the genes differentially expressed between Parasponia and Trema roots are several involved in mycorrhizal symbiosis as well as jasmonic acid biosynthesis. Measurements of hormone concentrations, showed that Parasponia and Trema roots harbor a difference in jasmonic acid/salicylic acid balance. However, mutants in jasmonic acid biosynthesis are unaffected in nodule development. Therefore, it remains a challenge to determine whether the difference in root transcriptomes between Parasponia and Trema are relevant in a symbiotic context.

In Chapter 7, I review hormone function in nitrogen-fixing nodule symbioses in legumes, Parasponia and actinorhizal species. In this chapter, I question whether different nodulating lineages recruited the same hormonal networks to function in nodule formation. Additionally, I discuss whether nodulating species harbor genetic adaptations in hormonal pathways that correlate with nodulation capacity. 


\section{Samenvatting}

Voor hun groei en ontwikkeling zijn planten afhankelijk van de beschikbaarheid van voedingsstoffen in de bodem. Een belangrijke voedingsstof voor de groei van planten is gebonden stikstof. Regelmatig is, onder natuurlijke omstandigheden, de hoeveelheid gebonden stikstof in de bodem limiterend, wat leidt tot een reductie in groei. Echter, sommige planten hebben zich gedurende de evolutie aangepast, waardoor de planten goed kunnen overleven op stikstof-arme bodems. Een bekend voorbeeld hiervan zijn planten uit de vlinderbloemigen familie. Bekende gewassen die tot deze familie behoren zijn erwten, bonen en soja. Wanneer vlinderbloemigen groeien op bodems die weinig stikstof bevatten vormen deze planten speciale orgaantjes op hun wortels, die wortelknolletjes genoemd worden. Een deel van de cellen in deze wortelknolletjes zijn volledig gevuld met stikstofbindende rhizobium bacteriën. Rhizobium bacteriën zijn instaat om stikstof gas uit de lucht op te nemen en dit om te zetten in ammonium, een gebonden vorm van stikstof. Het gevormde ammonium wordt door de bacteriën uitgescheden waardoor dit beschikbaar komt voor de plant. Op deze manier zijn vlinderbloemigen onafhankelijk van de beschikbare hoeveelheid stikstof in de bodem en kunnen deze planten groeien op plekken waar de meesten planten niet kunnen overleven.

De relatie, ook wel symbiose genoemde, met stikstofbindende rhizobium bacteriën is niet uniek voor vlinderbloemigen. Tropische bomen uit het genus Parasponia zijn ook instaat om een symbiose aan te gaan met rhizobium, waarbij wortelknolletjes worden gevormd. Parasponia is een relatief klein genus dat bestaat uit vijf soorten en behoort tot de hennepfamilie. Daarnaast kunnen nog ongeveer acht groepen van planten, afkomstig uit verschillende families, een stikstofbindende wortelknol symbiose aangaan met bacteriën uit het genus Frankia. Deze planten worden gezamenlijk ook wel actinorhiza planten genoemd. De gezamenlijke voorouder van zowel de vlinderbloemigen, actinorhizas en Parasponia leefde zo'n honderd miljoen jaar geleden, wat suggereert dat de verschillende wortelknol symbioses grotendeels onafhankelijk geëvolueerd zijn.

Het ontrafelen van de ontstaansgeschiedenis van stikstofbindende symbioses kan belangrijk inzicht bieden in de evolutionaire processen die ten grondslag liggen aan het ontstaan van nieuwe kenmerken. Daarnaast kan dit mogelijk een ingang bieden om dit kenmerk ooit in te bouwen in belangrijke landbouwgewassen, zoals rijst of aardappel. Tijdens mijn promotieonderzoek heb ik een bijdrage geleverd aan het begrijpen van de rhizobium symbiose in zowel vlinderbloemigen en Parasponia. 
De meeste kennis van stikstofbindende symbioses is opgedaan door onderzoek aan twee vlinderbloemige soorten die gebruikt worden als model: Lotus japonicus en Medicago truncatula (hierna genoemd medicago). Dit heeft laten zien dat de vorming van wortelknolletjes begint nadat signaalstoffen van de bacterie herkent worden door receptoren van de plant. Deze signaalstoffen bestaan uit een keten van chitine moleculen gekoppeld aan een vetzuurstaart en worden kortweg LCO's genoemd. Een zelfde soort signaalmoleculen wordt ook geproduceerd door mycorrhiza schimmels. Deze schimmels zijn instaat om een symbiose aan te gaan met ongeveer $80 \%$ van alle landplanten. Onderzoek heeft aangetoond dat vlinderbloemigen voor de herkenning van rhizobium bacteriën gebruik maken van een set genen die ook gebruikt wordt voor het aangaan van de mycorrhiza symbiose. Dit suggereert dat tijdens de evolutie van rhizobium symbiose, planten een deel van de signaleringsnetwerken die gebruikt worden voor het aangaan van de mycorrhiza symbiose hebben hergebruikt voor het maken van stikstofbindende wortelknolletjes. Voor het herkennen van de signaalmoleculen van mycorrhiza schimmels en rhizobium bacteriën maken planten gebruik van speciale receptoren. Deze receptoren zijn nauw verwant aan receptoren die betrokken zijn bij de afweer tegen ziekteverwekkende schimmels. In hoofdstuk $\mathbf{2}$ van dit proefschrift bediscussieer ik hoe de herkenning van symbiotische schimmels en bacteriën verstrengeld is met de herkenning van ziekteverwekkende schimmels. Daarnaast bediscussieer ik hoe mycorrhizas en rhizobium mogelijk gebruik maken van LCO's om de afweerrespons van planten te onderdrukken. In een vooruitblik, speculeer ik over de mogelijke rol van plantenhormonen bij het omzeilen van de afweerrespons.

Plantenhormonen spelen een belangrijke rol als regulatoren van groei en ontwikkeling. Ook bij de ontwikkeling van wortelknolletjes spelen hormonen een belangrijke rol. Onderzoek aan vlinderbloemigen heeft laten zien dat voor de vorming van wortelknolletjes met name het hormoon cytokinine erg belangrijk is. Planten waarin een gen dat codeert voor een van de receptoren van cytokinine defect is, zijn niet meer instaat om wortelknolletjes te vormen. Dit betekent dat de signaleringscascade die geactiveerd wordt na herkenning van cytokinine een belangrijke rol speelt in het proces van wortelknolvorming. Echter het was niet bekend hoe deze signaleringscascade geactiveerd wordt na herkenning van rhizobium bacteriën en hoe dit precies bijdraagt aan de vorming van stikstofbindende wortelknolletjes. In hoofdstuk 3 laat ik zien dat binnen drie uur na herkenning van rhizobium LCO moleculen een grote verandering in genexpressie (activiteit van genen) plaatsvind. Een groot deel van deze genen veranderd niet of nauwelijks in expressie in planten die ongevoelig zijn voor cytokinine, wat het belang van cytokinine signalering aangeeft vroeg tijdens het proces van knolvorming. Onder de genen die een verhoogde expressie laten zien in zowel het wild type (natuurlijk voorkomende genotype van een plant) als planten die ongevoelig zijn 
voor cytokinine bevinden zich genen die betrokken zijn bij de productie van cytokinine. Dit suggereert dat mogelijk de productie van cytokinine wordt verhoogd na herkenning van rhizobium bacteriën. In lijn met deze waarneming, laten metingen van de cytokinine concentratie zien dat de hoeveelheid cytokinine toeneemt binnen drie uur nadat medicago wortels zijn behandeld met LCO moleculen. Op basis hiervan beargumenteer ik dat de ophoping van cytokinine een belangrijke stap vormt in het proces van knolvorming.

Strigolactonen zijn een klasse van plantenhormonen waarvan de productie wordt verhoogd wanneer de beschikbaarheid van voedingsstoffen in de bodem limiterend is. Voorafgaand onderzoek heeft laten zien dat voor deze verhoging de activiteit van twee eiwitten essentieel is. Deze twee eiwitten, NSP1 en NSP2 genaamd, behoren tot de familie van GRAStype transcriptie factoren. Dit zijn eiwitten die de expressie van andere genen reguleren. Onderzoek aan rijst en medicago heeft aangetoond dat NSP1 en NSP2 betrokken zijn bij de regulatie van het gen DWARF27 (D27). Dit gen codeert voor een enzym dat verantwoordelijk is voor de eerste stap in de productie van strigolactonen. In vlinderbloemigen zijn NSP1 en NSP2 ook essentieel voor de vorming van wortelknolletjes. Om deze reden heb ik de vraag gesteld of de NSP1-NSP2-D27 module ook een rol speelt tijdens knolvorming. In hoofdstuk 4 laat ik zien dat in medicago de expressie van het MtD27 (D27 gen van medicago) gen verhoogd wordt binnen enkele uren nadat wortels behandeld zijn met rhizobium LCO moleculen. Daarnaast laat ik zien dat de expressie van MtD27 voornamelijk plaatsvind in de cellen die zich gaan delen en op deze manier bijdragen aan de vorming van het knolletje. In een later stadium, beperkt de expressie van MtD27 zich tot de delende cellen aan de bovenkant van het knolletje die er voor zorgen dat het knolletje blijft groeien (meristeem genoemd) en de cellen die geïnfecteerd worden door rhizobium. Analyse van de expressie van twee andere genen die ook betrokken zijn bij de productie van strigolactonen, MtCCD7 en MtCCD8, laat zien dat deze genen op dezelfde plekken tot expressie komen als MtD27. Daarnaast laat ik zien dat NSP1 en NSP2 essentieel zijn voor de verhoging van de expressie van MtD27 na herkenning van LCOs. Dit suggereert dat in de vlinderbloemigen de NSP1NSP2-D27 module gerekruteerd is voor een functie tijdens knolvorming.

Onderzoek aan vlinderbloemigen heeft veel inzicht opgeleverd in de moleculaire mechanismes die een rol spelen tijdens het proces van knolvorming. Het is echter niet bekend in hoeverre Parasponia en actinorhiza planten ook gebruik maken van deze mechanismes voor het vormen van stikstofbindende wortelknolletjes. Om hier inzicht in te verkrijgen is vergelijkend onderzoek nodig. Het laboratorium waar ik mijn onderzoek heb verricht is recent begonnen met onderzoek aan Parasponia, de enige niet-vlinderbloemige die instaat is om een wortelknol symbiose met rhizobium aan te gaan. Echter, om de functie van specifieke 
genen te kunnen onderzoeken is het belangrijke om snel en efficiënt genen te kunnen uitschakelen. Een techniek die hiervoor gebruikt kan worden is CRISPR/Cas9. Met deze techniek kan op hele specifieke plaatsen in het genoom (volledige DNA volgorde aanwezig ik elke cel) een breuk worden aangebracht. Deze breuk wordt vervolgens door de plantencel zelf gerepareerd, maar hierbij treden vaak kleine foutjes op waardoor het gen niet langer functioneel is. In hoofdstuk 5 beschrijf ik een protocol waarmee de CRISPR/Cas9 methode gebruikt kan worden voor het aanbrengen van mutaties (verandering in de DNA volgorde) in het genoom van Parasponia. Met deze methode is het mogelijk om, binnen een periode van drie maanden, plantjes te genereren waarin één of enkele genen niet meer functioneel zijn. Als proof-of-concept, heb ik een aantal genen gemuteerd (uitgeschakeld) waarvan bekend is dat ze in vlinderbloemigen essentieel zijn voor het maken van wortelknolletjes. Deze genen - PanHK4, PanEIN2, PanNSP1 en PanNSP2 - zijn respectievelijk betrokken bij de perceptie van cytokinine, signalering van het plantenhormoon ethyleen en het reguleren van de productie van strigolactonen. Planten die mutaties bevatten in PanHK4 of PanEIN2 laten een verschil in ontwikkeling zien ten opzichte van planten die geen mutaties bevatten. Deze verschillen komen overeen met wat is gevonden in andere planten. Echter, het effect van het uitschakelen van deze twee genen op wortelknolvorming verschilt van het effect in vlinderbloemigen. Dit suggereert dat de hormonen cytokinine en ethyleen mogelijk een verschillende rol spelen tijdens wortelknolvorming in vlinderbloemigen en Parasponia. Anderzijds leidt het uitschakelen van PanNSP1 en PanNSP2 wel tot het zelfde effect als eerder waargenomen in vlinderbloemigen, namelijk de afwezigheid van wortelknolletjes. Dit geeft aan dat NSP1 en NSP2 belangrijk zijn voor het maken van wortelknolletjes in zowel Parasponia en vlinderbloemigen, wat duidt op een geconserveerde functie. Tevens illustreert dit de kracht van onze aanpak. Alles bij elkaar laten mijn resultaten zien dat de methode dit ik ontwikkeld heb goed gebruikt kan worden voor het onderzoeken van de functie van specifieke genen in de tropische boom Parasponia.

Parasponia bomen zijn zeer nauw verwant aan tropische bomen uit het genus Trema. In tegenstelling tot Parasponia, zijn Trema bomen echter niet instaat om stikstofbindende wortelknolletjes te vormen. In hoofdstuk 6 heb ik gebruikt gemaakt van de nauwe verwantschap tussen Parasponia en Trema om te onderzoeken of het kunnen vormen van stikstofbindende wortelknolletjes gepaard gaat met een verandering in genexpressie. Om dit te kunnen doen, heb ik de expressie van alle genen in de wortels van twee Parasponia soorten en drie Trema soorten bepaald. Dit heb ik gedaan met een techniek die RNAseq genoemd wordt. De uitkomsten van dit experiment lieten zien dat de expressie van genen in de wortels van Parasponia verschilt van dat in Trema wortels. Onder de genen die anders tot expressie komen in Parasponia wortels ten opzichte van Trema wortels zijn 
genen die een bekende rol vervullen in de symbiose met mycorrhiza schimmels en genen die betrokken zijn bij de productie van het plantenhormoon jasmonzuur. Metingen van de hormoonconcentratie laten zien dat Parasponia wortels ook inderdaad meer jasmonzuur bevatten in vergelijking met Trema wortels. Echter, planten die gemuteerd zijn zodat ze niet meer instaat zijn om jasmonzuur te produceren kunnen nog steeds functionele wortelknolletjes vormen. Daarom is het op dit moment nog niet duidelijk wat het verschil in genexpressie tussen Parasponia en Trema precies betekent voor de stikstofbindende symbiose tussen Parasponia en rhizobium.

In hoofdstuk 7 bediscussieer ik de resultaten van mijn promotieonderzoek en plaats ik dit in een bredere context. 


\section{ACKNowledgements}

I would like to express my gratitude to everyone that has helped or supported me in any way during the duration of $\mathrm{my} \mathrm{PhD}$ research. A few people I would like to mention in person.

Allereerst wil ik mijn dagelijkse begeleider en copromotor, Rene, van harte bedanken voor de kansen en het vertrouwen dat jij mij hebt geboden. Jij stond altijd voor mij klaar wanneer ik vragen had of behoefte had aan een klankboord wanneer ik twijfelde over de juiste onderzoeksstrategie. Ik kijk met veel plezier terug op de vele wetenschappelijke discussies die wij samen hebben gevoerd. Daarnaast ben ik jou erg dankbaar voor de ondersteuning die jij mij hebt geboden in het bepalen van de volgende stap in mijn carrière.

Ook wil ik mijn promotor Ton bedanken. Jij begeleide als directeur van EPS het talentenprogramma waaraan ik heb meegedaan. Nadat afloop van dit programma hebben wij een zeer prettig gesprek gevoerd. Hierin gaf ik aan dat ik een promotie bij MolBi als een interessante optie zag. De volgende dag kreeg ik een email van Rene waarin hij aangaf dat er op korte termijn mogelijk een plek bij MolBi beschikbaar zou komen. Uiteindelijk heb jij er zo mede voor gezorgd dat ik bij MolBi mijn promotieonderzoek heb kunnen uitvoeren. Ook wil ik jou bedanken voor de suggesties voor verbetering van mijn onderzoek en de hulp bij het opschrijven van mijn stellingen.

Wouter, bedankt dat je mijn "externe" begeleider wilde zijn. Onze gesprekken en het advies dat jij mij gegeven hebt tijdens de eerste jaren van mijn promotieonderzoek hebben mij zeer geholpen. Halverwege mijn promotietraject kwam jij naar MolBi en werd "extern" wel een beetje een relatief begrip. Jij hebt deze taak met veel toewijding en inspanning uitgevoerd, dank daarvoor! Sinds jouw komst naar MolBi heb jij mij geholpen met het afronden van mijn werk aan medicago. Dit heeft uiteindelijk geleid tot twee mooie publicaties. Ook heb $i k$ genoten van onze middagwandelingen. Ik wens je veel succes met het vinden van een geschikte plek om jouw wetenschappelijke carrière verder vorm te kunnen geven.

Henk, bedankt voor jouw wijze woorden. Vooral in het eerste en laatste jaar van mijn promotietraject heeft dit mij zeer geholpen. Jij begreep de problemen waarmee ik worstelde en wist dit in perspectief te plaatsen. Dit gaf mij de rust en het vertrouwen om door te zetten.

Sidney en Marijke, zonder jullie hulp was het waarschijnlijk niet gelukt om een stabiel transformatie protocol in combinatie met CRISPR/Cas-mutagenese op te zetten voor Parasponia. Marijke, jouw expertise en ervaring met weefselkweek was zeer belangrijk. 
Sidney, jij hebt mij met bijna al mijn Parasponia werk geholpen de laatste twee jaar van mijn onderzoek. Het was voor mij dan ook niet meer dan logisch om jou te vragen mij ook bij de laatste stap van mijn promotietraject te helpen. Bedankt dat jij mijn paranimf wilde zijn! Ik wil jullie daarnaast allebei heel hartelijk bedanken voor al het harde werk. Zonder jullie had mijn proefschrift er heel anders uitgezien!

Erik, bedankt voor de succesvolle samenwerking. Samen met jou en Rene hebben wij een 'Annual Review' geschreven. Jij hebt hier een zeer grote bijdrage aan geleverd.

Rik op den Camp, Eva, Tanya and Harro thanks for your contributions to chapter 3. Rik ik kwam in de groep op het moment dat jij je proefschrift aan het afronden was. Ik had de eer om verder te gaan met jouw project aan cytokinine. Dit heeft uiteindelijk geresulteerd in een mooie publicatie in Molecular Plant. Tanya, I am grateful for all your help with cytokinin quantification. You always made time to analyse my samples and explain the results patiently. Eva, ik wil jou bedanken voor de inspanning om ons werk te integreren. Helaas zagen de reviewers dit anders.

Wei and Ting Ting, thanks for the collaboration on DWARF27. It was a struggle to get this work published but we managed in the end! I am very proud of our paper in BMC Plant Biology. Also, I would like to thank the both of you for your help with introducing me to the MolBi lab. You both helped me find my way when I just started my PhD research. Ting Ting, I also want to thank you four friendship, especially during the first years of my PhD. Finally, I like to wish you both a lot of success in your future careers!

I would like to thank all members of the Parasponia team. Both current members - Robin, Rens, Kerstin, Titis, Yuda, Luuk, Fengjiao, Jan V, Kana, Simon, Sidney, Marijke, Wouter and Rene - as well as past members of the team - Maryam, Trupti, Elena and Cao - I am grateful to you all. Robin and Rens, thanks for your help with the bioinformatics part of my PhD research. This was very important for my efforts to compare Parasponia and Trema root transcriptomes and would have been impossible without. Kerstin and Wouter, thanks for your help with hormone measurements! Fengjiao, Titis, Luuk and Maryam, thanks for your contributions to chapter 5 of my thesis. Your contributions helped to transform this chapter from a mere method description into a real story. I hope we will be able to get this work published soon. Titis, I enjoyed our evening tea breaks and shared dinners after working late. Jan V, I would like to thank you for all your help with RNA isolation and help with resolving cloning issues. Elena, many thanks for all your help with microscopic analyses of my Parasponia nodules. Cao, thank you for teaching me how to work with Parasponia. 
I am also grateful to all the BSc. and MSc. students that I had the pleasure to supervise during my PhD project. Malaika, Özhan, Jonathan, Susan, Titis, Max, Fleur, Luuk, Roos, Maikel, Sidney, Anneke, Fenne, Willem-Jan, Matthijs and Jasper, each and every one of you contributed to my research. I learned a lot from working with you and I hope that you all enjoyed your time at MolBi as much as I did working with you! I hope your training allows you to have a blooming future career.

I would like to thank Maria and Marie-Jose as well as the MolBi lab managers team - Jan Ho, Jan V, Carolien, Olga, Henk, Marijke and Sidney - for all their work 'behind the scenes' to make research at MolBi possible.

I am also grateful to all students, PhD students and staff at Molbi for a great atmosphere. I know I sometimes made your lives difficult with all the critical question I asked during work discussion. I hope that in the end my questions were helpful to improve your research. Also, I would like to thank our neighbors from Cell Biology and Plant Developmental Biology for the nice coffee breaks, lunch breaks and of course lab trips.

I would like to thank my fellow PhD-students: Rik Huisman, Ting Ting, Huchen, Guiling, Defeng, Jianyong, Fengjiao, Xu, Jelle, Rens, Jieyu, Yinshan, Martinus, Luuk, Yuda, Asma, Amina, Peng, Titis, Tian, Ole, Sabine, Adam, Bandan, Jeroen, Aniek, Juliane, Maryam, Trupti, Wei, Sergey, Aleksandr and Sjef. Your companionship made working in the lab very enjoyable. Also, I really enjoyed all the times we went for drinks together on Friday evening. Rik, Sabine, Bas and Ting Ting, together we travelled to Czech to visit Adam's wedding. I very much enjoyed our trip together. Adam, thanks for inviting us to your wedding. Guiling, you were always there on weekends. Thanks for keeping me company during, otherwise lonely, weekend lunch breaks. Finally, I like to wish all of you good luck with finishing your theses and I wish you all the best for your future careers.

Tot slot wil ik mijn familie en vrienden bedanken voor alle steun en afleiding. Pap en mam, jullie hebben ons in alles gesteund, dank daarvoor! Tessa, Pieter-Harm en Jos, ook jullie bedankt voor de steun en afleiding tijdens weekenden. Jos, bedankt dat jij mijn paranimf wilt zijn! 


\section{List of Publications}

Robin van Velzen, Rens Holmer, Fengjiao Bu, Luuk Rutten, Arjan van Zeijl, Wei Liu, Luca Santuari, Qingqin Cao, Trupti Sharma, Defeng Shen, Yuda Roswanjaya, Titis Wardhani, et al. (2017) Parallel loss of symbiosis genes in relatives of nitrogen-fixing non-legume Parasponia. bioRxiv DOI: 10.1101/169706

Ahmed Abd-El-Haliem, Jack Vossen, Arjan van Zeijl, Sara Dezhsetan, Christa Testerink, Michael Seidl, Martina Beck, James Strutt, Silke Robatzek and Matthieu Joosten (2016) Biochemical characterization of the tomato phosphatidylinositol-specific phospholipase C (PI-PLC) family and its role in plant immunity. Biochim. Biophys. Acta 1861: 1365-1378

Erik Limpens*, Arjan van Zeijl ${ }^{*}$ and Rene Geurts* (2015) Lipo-chitooligosaccharides modulate plant host immunity to enable endosymbioses. Annu. Rev. Phytopathol. 53: 311-334

Arjan van Zeijl, Rik op den Camp, Eva Deinum, Tatsiana Charnikhova, Henk Franssen, Huub op den Camp, Harro Bouwmeester, Wouter Kohlen, Ton Bisseling and Rene Geurts (2015) Rhizobium lipo-chitooligosaccharide signaling triggers accumulation of cytokinins in Medicago truncatula roots. Mol. Plant 8: 1213-1226

Arjan van Zeijl*, Wei Liu*, Ting Ting Xiao*, Wouter Kohlen, Wei-Cai Yang, Ton Bisseling and Rene Geurts (2015) The strigolactone biosynthesis gene DWARF27 is co-opted in rhizobium symbiosis. BMC Plant Biol. 15: 260

Carolien Ruyter-Spira*, Wouter Kohlen*, Tatsiana Charnikhova, Arjan van Zeijl, Laura van Bezouwen, Norbert de Ruijter, Catarina Cardoso, Juan Antonio Lopez-Raez, Radoslava Matusova, Ralph Bours, Francel Verstappen, and Harro Bouwmeester (2011) Physiological effects of the synthetic strigolactone analog GR24 on root system architecture in Arabidopsis: another belowground role for strigolactones? Plant Physiol. 155: 721-734

Arjan van Zeijl, Titis Wardhani, Maryam Seifi-Kalhor, Luuk Rutten, Fengjiao Bu, Marijke Hartog, Sidney Linders, Elena Fedorova, Ton Bisseling, Wouter Kohlen and Rene Geurts CRISPR/Cas9-mediated mutagenesis of four putative symbiosis genes of the tropical tree Parasponia andersonii reveals novel phenotypes. Manuscript in preparation.

\footnotetext{
${ }^{*}$ Shared first authorship
} 


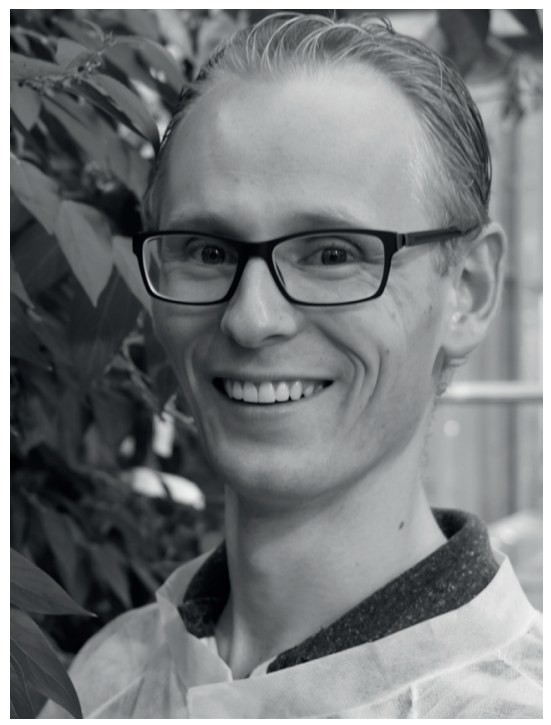

\section{CURRICULUM VITAE}

Adrianus Leonardus (Arjan) van Zeijl was born on the $27^{\text {th }}$ of September 1986 in the Noordoostpolder, the Netherlands. After obtaining his high school degree from the "Emelwerda college" in Emmeloord, the Netherlands, he moved to Wageningen in 2005 to start his university education at Wageningen University. There, he enrolled in the Plant Science bachelor programme, from which he graduated cum laude in 2008. During his bachelor studies, he became interested in the molecular aspects of plant biology. This led him to decide to continue his academic education with an MSc in Plant Biotechnology. His first thesis project was performed at the Laboratory of Plant Physiology, under supervision of Dr Carolien Ruyter-Spira and Prof. Dr Harro Bouwmeester. During this time, he studied how the plant hormone strigolactone affects auxin homeostasis in Arabidopsis thaliana. In 2010, his thesis report was awarded the Rijk Zwaan Plant Science Award for the best plant sciencerelated thesis defended at a Dutch University. He performed his second thesis project at the Laboratory of Phytopathology, under supervision of Ahmed Abd-El-Haliem and Dr Matthieu Joosten. His thesis focussed on the biochemical characterization of several phospholipase $\mathrm{C}$ isoforms from tomato, which included determining the most optimal $\mathrm{pH}$ and calcium concentration for in vitro enzyme activity. After completion of his second thesis, he decided to extend his Master programme and move to Cologne, Germany for an internship at the Max Planck Institute for Plant Breeding Research. There, he joined the group of Prof. Dr Maarten Koornneef and worked under supervision of Dr Glenda Willems on validation of candidate loci that were identified from Genome-Wide Association and Quantitative Trait Locus studies for freezing tolerance in A. thaliana. In the summer of 2011, he completed his Master degree for which he graduated cum laude. Later that year, Arjan joined the Laboratory of Molecular Biology at Wageningen University to perform his PhD research. Under supervision of Dr Rene Geurts and promoter Prof. Dr Ton Bisseling, he studied how legumes and Parasponia engage in symbiosis with nitrogen-fixing rhizobium bacteria. The results of his research are presented in this thesis. 


\title{
Education Statement of the Graduate School Experimental Plant Sciences
}

\author{
Issued to: Arjan van Zeijl \\ Date: 18 October 2017 \\ Group: $\quad$ Laboratory of Molecular Biology \\ University: Wageningen University \& Research
}

1) Start-up phase

- First presentation of your project

Title: Role of cytokinin in the evolution of Parasponia root nodule formation

Writing or rewriting a project proposal

Title: Parasponia; the key to identify constraints in developmental mechanisms underlying rhizobium nodulation

- Writing a review or book chapter

Title: Lipochitooligosaccharides modulate plant host immunity to enable endosymbioses. (2015) Annual Review of Phytopathology 53, 311-334.

MSc courses

Advanced Bioinformatics (BIF-30806) Laboratory use of isotopes

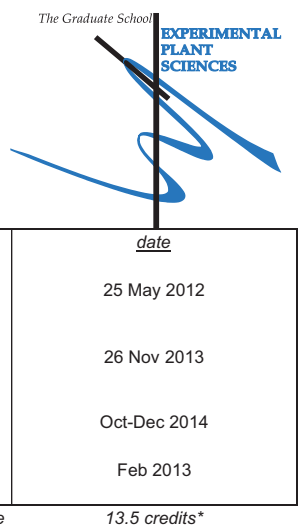

\section{2) Scientific Exposure}

- EPS PhD student days

European Plant Science retreat for PhD students, JIC, Norwich, UK

EPS PhD student day, Amsterdam, NL

ExPectationS day 2013; Inspiration and Creativity in science, Wageningen, NL

European Plant Science retreat for PhD students, VIB, Ghent, Belgium

EPS Student day, Leiden, NL

EPS PhD student day 'Get2Gether', Soest, NL

- EPS theme symposia

EPS theme 1 'Developmental Biology of Plants', Wageningen, NL

EPS theme 1 'Developmental Biology of Plants', Leiden, NL

EPS theme 1 'Developmental Biology of Plants', Wageningen, NL

EPS theme 1 'Developmental Biology of Plants', Wageningen, NL

- National meetings (e.g. Lunteren days) and other national platforms

Annual meeting 'Experimental Plant Sciences', Lunteren, NL

Annual meeting 'Experimental Plant Sciences', Lunteren, NL

Annual meeting 'Experimental Plant Sciences', Lunteren, NL

Annual meeting 'Experimental Plant Sciences', Lunteren, NL, co-chair 'Hormones' session

Annual meeting 'Experimental Plant Sciences', Lunteren, NL, co-chair 'Comparative biology and evolution' session

- Seminars (series), workshops and symposia

Seminar Dr. Veronica Grieneisen

Seminar Prof. Dr. Ottoline Leyser; 'Auxin, self-organisation, and the colonial nature of plants'

Seminar Prof. Dr. Steffen Abel; 'Phosphate sensing in root development'

Seminar Prof. Dr. Hong Ma; 'Molecular genetic, transcriptomic and genomic characterization of meiotic

recombination in Arabidopsis'

Seminar Dr. Pierre Hilson; 'Peptide hormones regulate plant tropic growth and root development'

Seminar Prof. Sir David Baulcombe; 'Plant versus virus: defense, counter defense and counter counter defense'

Seminar Prof. Sugimoto; 'Strigolactones, new plant hormones. Importance of their stereochemistry for bioactivity as qermination stimulant'

Mini-symposium 'Frontiers in plant morphogenesis'

Seminar Prof. Ruth Finkelstein; 'ABA signaling networks in Arabidopsis'

Seminar Andrew Sugden; 'Writing for high impact journals'

Seminar Dr. Andreas Niebel; 'The CCAAT-box binding factors MtNF-YA1\&2 are transcriptional regulators required for Nod factor signaling, rhizobial infection and nodule development'

Seminar Prof. Rüdiger Simon; 'Plant stem cell systems'

Seminar Prof. Eric Kramer; 'Auxin and plasmodesmata; measuring fluxes in the root'

Seminar Prof. Hanhui Kuang; 'Using the Nicotiana-TMV system to study resistance gene evolution and plant genome stability'

PDB start symposium, Wageningen, NL

Seminar 'How to write a world class paper', Wageningen Library

Seminar Prof. Jiayang Li; 'Understanding the molecular mechanisms underlying rice tillering'

Plant Sciences Seminar: Statistics, genetics and omics in research

Seminar Dr. Jos Raaijmakers; 'Back to the roots'

Seminar Dr. Andrea Bräutigam; 'From 'Omics' to single gene analyses: "Case studies in C4 photosynthesis

using next generation sequencing"'

WPC lecture; 'The secret of a successful PhD: Making the implicit explicit'

Plant Sciences Seminar: Mutualism in action, Prof. Toby Kiers and Dr. Rene Geurts

Seminar Dr. Melissa Wong; 'Novel insights into genome evolution of legumes -genome comparison, WGD prediction and reconstruction of ancestral genomes'

Lecture Prof. Hans Clevers; 'The future of science'

Plant Sciences Seminar: Bioinformatics Prof. Dick de Ridder, Paul Kersey

Seminar Prof. Marilyn Roossinck; 'Plant virus ecology and evolution: pathogens to mutualists' 
EPS flying seminar Prof. Dr. George Coupland; 'Seasonal flowering in annual and perennial plants' WEES seminar Prof. Kevin Foster; 'The evolution of cooperation and competition in microbes'

Seminar Prof. Dr. Jeff Doyle; 'Polyploidy in wild relatives of soybean and other legumes: systematics, comparative and functional genomics, and nodulation'

Seminar Dr. Siobhan Brady; 'Regulation of root morphogenesis in tomato species in the face of a changing environment

WEES seminar Prof. Marc-Andre Selosse; 'Mycoheterotrophy and mixotrophy : plants eating mycorrhizal fungi

Seminar Dr. Alain Goossens; 'How jasmonates provide the key to harness plant chemistry'

Seminar Prof. Dr. Jane Parker; 'Plant intracellular immunity: evolutionary and molecular underpinnings'

Symposium 'Evolution and maintenance of (belowground) cooperation', VU University, Amsterdam

Seminar Dr. Jan Ruijter; 'Analysis of qPCR data. The use and usefulness of amplification curve analysis'

Farewell Symposium EPS director Ton Bisseling; 'The Undergrond Labyrinth: Roots, Friends and Foes'

\section{Seminar plus}

Seminar Prof. Dr. Jeff Doyle; 'Polyploidy in wild relatives of soybean and other legumes: systematics,

comparative and functional genomics, and nodulation'

Seminar Dr. Siobhan Brady; 'Regulation of root morphogenesis in tomato species in the face of a changing environment

Seminar Dr. Alain Goossens; 'How jasmonates provide the key to harness plant chemistry'

- International symposia and congresses

Plant Molecular Biology Gordon Research Seminar, Holderness, New Hampshire, USA

Plant Molecular Biology Gordon Research Conference, Holderness, New Hampshire, USA

EMBL PhD symposium, Heidelberg, Germany; 'Exploring Nature's toolbox'

Mini-symposium: "Evolution of Nodulation", Munich, Germany

BNF Non-legume satellite symposium, Budapest, Hungary

Genomics of nitrogen-fixing organisms workshop, Budapest, Hungary

12th European nitrogen fixation conference, Budapest, Hungary

\section{- Presentations}

Poster: Summerschool Natural Variation, Wageningen, NL

Poster: Lunteren meeting 2013, Lunteren

Poster: EPSR 2013, VIB, Ghent (re-use)

Talk: PDB start symposium, Wageningen, NL; 'Hormones and symbiosis'

Invited talk: Max-Planck Institute, Cologne, Germany; 'Medicago nodule formation is preceded by cytokinin

biosythesis'

Talk: NWO Lunteren meeting 2014, Lunteren; 'Medicago nodule formation is preceded by cytokinin

biosythesis'

Poster: Plant Molecular Biology Gordon Research Seminar+Conference, Holderness, New Hampshire

Poster: EMBL PhD symposium, Heidelberg, Germany; 'Parasponia; the key to solve the nitrogen problem'

Talk: EMBL PhD symposium, Heidelberg, Germany; 'Parasponia; the key to unravel the evolutionary

trajectory towards a nitrogen-fixing symbiosis'

Poster: Mini-symposium "Evolution of Nodulation", Munich, Germany (re-use)

Talk: EPS Get2Gether 2015; Rhizobium lipo-chitooligosaccharide signaling triggers accumulation of

cytokinins in Medicago truncatula roots

Talk: 12th European Nitrogen fixation conference, Budapest, Hungary

- IAB interview

Meeting with a member of the International Advisory Board of EPS

$\checkmark$ Excursions

Excursion to Plant Breeding Company Rijk Zwaan, de Lier

19 Jan 2015

22 Jan 2015

12 May 2015

09 Sep 2015

26 Nov 2015

08 Dec 2015

21 Jan 2016

04 Feb 2016

14 Mar 2016

08 Feb 2017

12 May 2015

09 Sep 2015

08 Dec 2015

19-20 Jul 2014

20-25 Jul 2014

23-25 Oct 2014

07-08 Jan 2015

23-24 Aug 2016

25 Aug 2016

25-28 Aug 2016

21-24 Aug 2012

22-23 Apr 2013

24-26 Aug 2013

14 Oct 2013

20 Feb 2014

15 Apr 2014

19-25 Jul 2014

23-25 Oct 2014

23-25 Oct 2014

07-08 Jan 2015

29-30 Jan 2015

25-28 Aug 2016

29 Sep 2014

27 Sep 2013

28.3 credits *

\section{3) In-Depth Studies}

\section{- EPS courses or other PhD courses}

Summerschool 'Natural variation of plants', Wageningen, NL

Course 'Current Trends in Phylogenetics', Wageningen, NL

Course 'The power of RNA seq', Wageningen, NL

- Journal club

Journal club Molecular Biology, WUR

Individual research training

\section{$\underline{\text { date }}$}

21-24 Aug 2012

22-26 Oct 2012

05-07 Jun 2013

2011-2015

Subtotal In-Depth Studies

6.6 credits*

\section{4) Personal development}

- Skill training courses

$\mathrm{PhD}$ Competence Assessment

Project and Time Management

ExPectationS; Inspiration and Creativity in science (EPS Career Day), Wageningen, NL

Mobilising your -scientific- network

WGS training Career Development for Post Docs

- Organisation of PhD students day, course or conference

Organisation of pubquiz at EPS Get2Gether 2015

Membership of Board, Committee or PhD council

May-Jun 2016

29-30 Jan 2015

4.6 credits*
53.0

${ }^{*}$ A credit represents a normative study load of 28 hours of study. 
The research described in this thesis was financially supported by the Dutch Science Organization (NWO) - NWO-NSFC Joint Research project (846.11.005) and NWOVICI (865.13.001) - and the European Research Council (ERC-2011-AdG294790).

Financial support from Wageningen University for printing this thesis is gratefully acknowledged.

Cover design: Agilecolor Design Studio/Atelier (www.agilecolor.com)

Layout: Arjan van Zeijl

Printed by: GVO drukkers \& vormgevers B.V., Ede, NL 


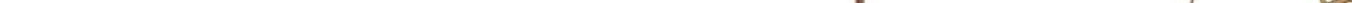

